\title{
FORMATION OF
}
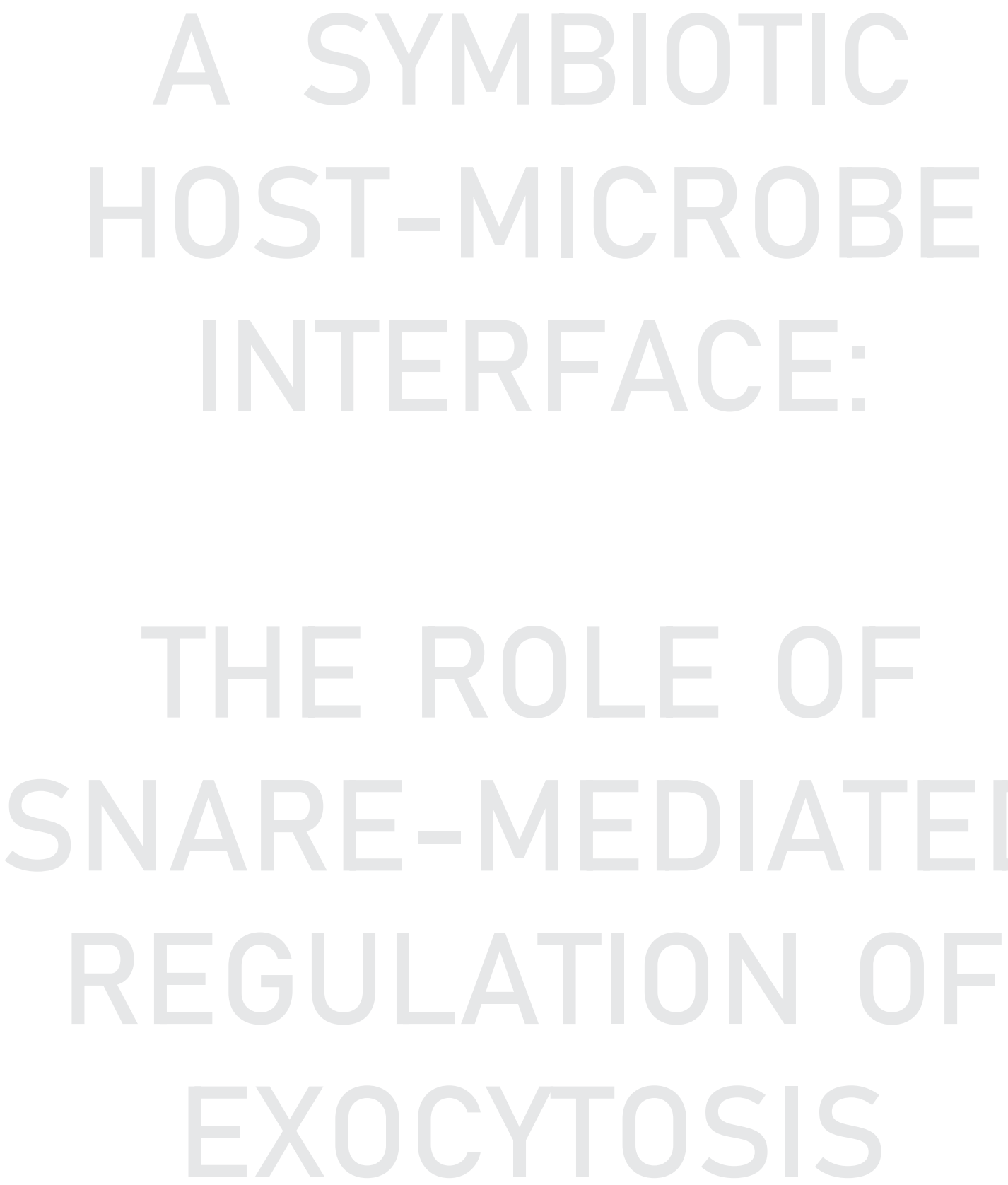

Rik HUISMAN 

Formation of a symbiotic host-microbe interface:
the role of SNARE-mediated regulation of exocytosis

Rik Huisman 


\section{Thesis commitee}

\section{Promotor}

Prof. Dr A.H.J. Bisseling

Professor of Molecular Biology

Wageningen University \& Research

\section{Co-promotor}

Dr E.H.M. Limpens

Assistant professor, Laboratorry of Molecular Biology

Wageningen University \& Research

\section{Other members}

Prof. Dr D. Weijers, Wageningen University \& Research

Prof. Dr C. Gutjahr, LMU, Munich, Germany

Dr M.A.C.J. Kwaaitaal, University of Amsterdam

Dr M.J. Ketelaar, Wageningen University \& Research

This research was conducted under the auspices of the Graduate School Experimental Plant Sciences 


\title{
Formation of a symbiotic host-microbe interface: the role of SNARE-mediated regulation of exocytosis
}

\author{
Rik Huisman
}

Thesis

submitted in fulfilment of the requirements for the degree of doctor at Wageningen university by the authority of the Rector Magnificus

Prof. Dr A.P.J. Mol, in the presence of the Thesis Committee appointed by the Academic Board to be defended in public on Friday 16 February 2018 at 4 p.m. in the Aula. 
Rik Huisman

Formation of a symbiotic host-microbe interface:

the role of SNARE-mediated regulation of exocytosis

158 pages.

PhD thesis, Wageningen University, Wageningen, the Netherlands (2018) With references, with summary in English

ISBN: 978-94-6332-317-8

DOI: https://doi.org/10.18174/432066 


\section{Table of Contents}

General Introduction

\section{Chapter 2}

Haustorium formation in Medicago truncatula roots infected by Phytophthora palmivora does not involve the common endosymbiotic program shared by AM fungi and rhizobia

\section{Chapter 3}

A symbiosis-dedicated SYNTAXIN OF PLANTS 13II isoform controls the formation of a stable host-microbe interface in symbiosis

\section{Chapter 4}

Functional redundancy among plant exocytotic SNARE proteins to form a symbiotic interface

\section{Chapter 5}

Specialization of SYP132a to control arbuscule functionality

\section{Chapter 6}

General Discussion

Summary

References

List of publications

Acknowledgements 


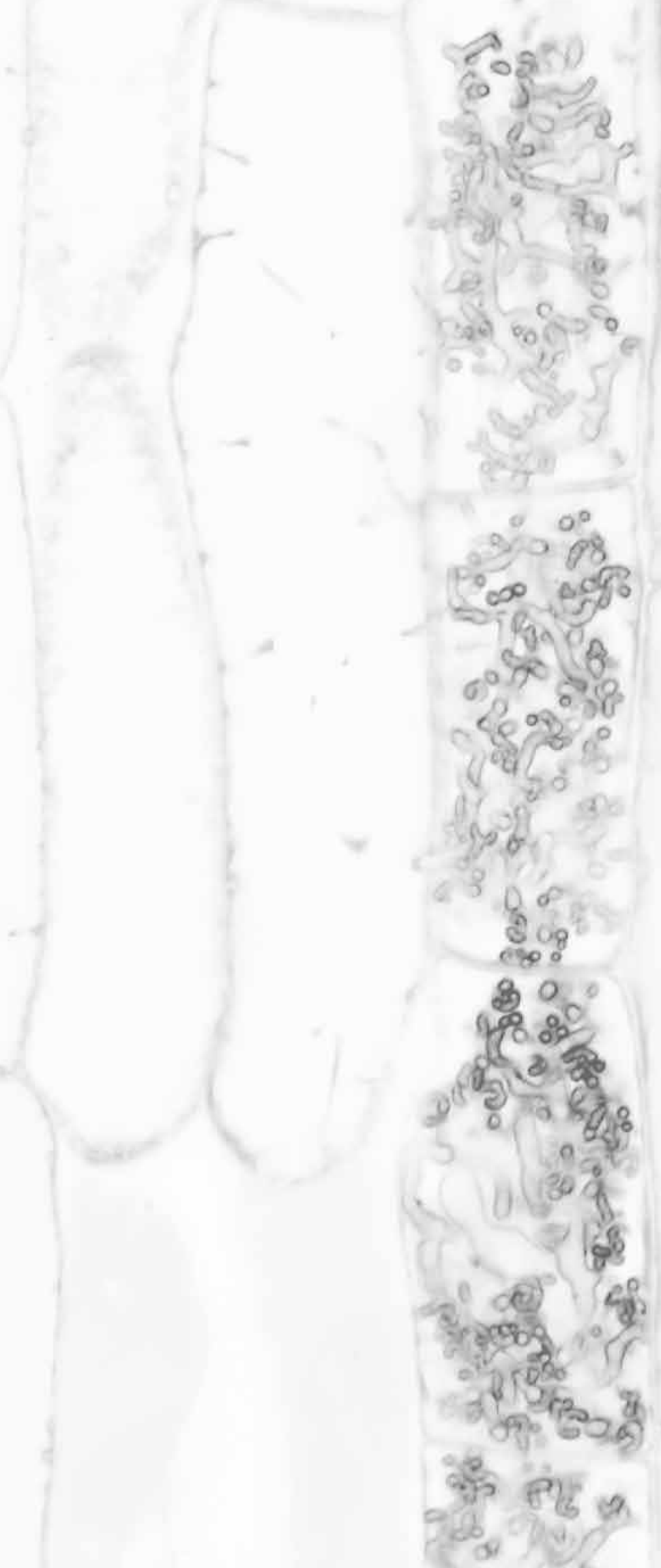




\section{Chapter 1}

General Introduction 


\section{Chapter 1}

Plants rely heavily on symbiosis with micro-organisms in the soil to increase their access to scarce nutrients. Especially nitrogen and phosphorous are elements that are growth limiting for plants in most ecosystems (Harpole et al., 2011). Symbiosis is an integral aspect of plant nutrition, as perhaps the only place were plants grow without microbes is the lab of a biologist. Symbiosis classically refers to any relationship of organisms living together, regardless of the resulting benefits or costs this brings for both partners. However, in this thesis I will use the term symbiosis to describe interactions of plants with microbes that are on average mutual beneficial for both partners. A common example is the interaction of plants with bacteria that are free-living in the soil that release nutrients from insoluble or organic compounds that remain otherwise inaccessible to the plant (Clarholm, 1985; Becquer et al., 2014). Plants actively promote these bacteria by secretion of mucilage containing among others carbohydrates and amino acids. Nevertheless, the commitment of both partners is relatively low since there is competition for compounds secreted by the plant and the released nutrients between all soil inhabitants. Endosymbiosis is a far more advanced and intimate form of symbiosis. During endosymbiosis, all or part of the microbe is hosted within the plant cell, which allows a targeted exchange of carbon and nutrients between the two partners. The most widespread and ancient form of endosymbiosis is the interaction of plants with arbuscular mycorrhizal (AM) fungi, which form an extension to the plant root system and help plant to take up nutrients. Many (if not all) other endosymbioses that evolved later make use of mechanisms and plant genes involved in AM symbiosis. A key example of this are nitrogen fixing rhizobia that interact with leguminous plants.

Most (if not all) microbes that enter living plant cells remain surrounded by a plant derived membrane. AM fungi form arbuscules in plant cells. Arbuscules are highly branched feeding structures that are surrounded by the peri-arbuscular membrane. Rhizobia are completely taken up in plant cells, forming (transient) $\mathrm{N}_{2}$-fixing organelles called symbiosomes, which are surrounded by the so-called peri-bacteroid membrane. These peri-microbial membranes are the main site of nutrient exchange between both partners and their protein composition is highly specialized for this role. This places the peri-microbial membranes at the heart of endosymbiosis as they establish an intimate host-microbe interface. Understanding the formation and maintenance of the peri-microbial membrane is therefore crucial to understand endosymbiosis. Recent studies have revealed that the formation of the peri-arbuscular membrane and the peri-bacteroid membrane depend on at least partially overlapping sets of plant genes (Huisman et al., 2012). Besides mutualist symbionts, also (hemi-)biotrophic filamentous pathogens infect living plant cells in which they are surrounded by a peri-microbial membrane. If symbiotic and pathogenic peri-microbial membrane formation depend on similar mechanisms, then understanding the mechanisms that guide accommodation of symbionts will also help understanding the mechanisms that guide accommodation of pathogens.

In this thesis, I will study how plant membrane trafficking is regulated to create a symbiotic host-microbe interface. Further, I will study whether the formation of a symbiotic and a pathogenic host-microbe interface depend on the same plant genes. 


\section{Arbuscules and arbuscular mycorrhiza symbiosis}

Most plants can engage in a symbiosis with filamentous fungi that transfer nutrients to the plant, in exchange for carbohydrates produced by the pant. This means that the fungi effectively form an extension to the plant root system, increasing the total volume of soil from which nutrients can be taken up. Mycorrhiza especially increase the uptake of nutrients that are limited by their low solubility and low diffusion in the soil, like phosphorous and zinc (Mosse, 1973; Smith \& Read, 2008). These nutrients are quickly depleted in the soil directly surrounding the plant root (Lewis \& Quirk, 1967). Mycorrhizal fungi extend beyond this zone and are less prone to create depletion zones themselves due to their small diameter. Mycorrhizal fungi also transfer more mobile nutrients like nitrogen to the plant (Leigh et al., 2009), but the importance of mycorrhiza for nitrogen nutrition seems to be limited (Smith \& Read, 2008). Besides increasing access to nutrients, mycorrhiza can enhance the uptake of water (Augé, 2001), or increase plant resistance to biotic stresses (Pozo et al., 2010).

There are different forms of mycorrhizal symbiosis: Ectomycorrhizal fungi form a symbiosis with many temperate forest trees. Ectomycorrhizal fungi do generally not enter living plant cells, but form a network of hyphae called 'Hartig net' that surrounds epidermal cells and which forms the host-microbe interface. The fungal partners that can form ectomycorrhiza are diverse (more than 20.000 species), as ectomycorrhiza evolved approximately 60 independent times from a wide range of saprotrophic ancestors (Martin et al., 2016). Endomycorrhizal fungi infect living plant cells. Some specific endomycorrhizal relations are formed by ericoid mycorrhiza and orchid mycorrhiza that are both restricted to interaction with a single plant family. Arbuscular mycorrhiza (AM) are the most ancient and widespread form of mycorrhizal symbiosis. AM fungi are all part of the phylum Glomeromycota, and as obligate biotrophs they fully depend on plants for their carbon supply. AM symbiosis dates back 450-460 million years, to the first plants that colonized land (Redecker et al., 2000; Wang et al., 2010b). Today, around $80 \%$ of all land plants is able to form a symbiosis with AM fungi.

The infection of plants by AM fungi starts with the formation of a hyphopodium on the plant root, from which a hypha emerges that penetrates an epidermal cell. The growth of hyphae through cells requires the active contribution of the plant. Preceding the fungal invasion, a pre-penetration apparatus is formed that consists of an ER- and cytoskeleton-rich cytoplasmic column that predicts the path of the invading fungus (Genre et al., 2005). After passing the epidermis, the fungus colonizes the root cortex by forming either inter- or intracellular hyphae. When the fungus reaches the inner cortical cell layers, arbuscules are formed. The plant cells harboring an arbuscule, as well as the arbuscules themselves are optimized for the exchange of carbon and nutrients: The membrane contains symbiosis dedicated phosphate transporters (Harrison et al., 2002; Yang et al., 2012; Breuillin-Sessoms et al., 2015), Ammonium transporters (Guether et al., 2009; Kobae 
et al., 2010), lipid transporters (Zhang et al., 2010a), and proton pumps that create a electrochemical potential across the membrane that energizes the transporters (Krajinski et al., 2014; Wang et al., 2014). The arbuscular cells increase their production of fatty acids, that are transferred to the fungus (Bravo et al., 2017; Jiang et al., 2017; Keymer et al., 2017; Luginbuehl et al., 2017).

Arbuscules are relatively short-lived structures that collapse after $\sim 2.5$ days (Alexander et al., 1988, 1989; Kobae \& Hata, 2010). After collapse, the fungal branches aggregate into a clump, that is digested by plant enzymes and encased by cell wall like material (Cox \& Sanders, 1974; Floss et al., 2017). The plant cell remains alive, and can be reinfected by a successive generation of arbuscules (Kobae \& Fujiwara, 2014). It is currently still unknown why arbuscules are so short lived. Measurements on the volume of plant cells occupied by the fungus suggest that arbuscules are either growing or collapsing, but never reach a stable phase in which mature arbuscules are maintained (Alexander et al., 1988, 1989). It has been shown that the collapse of arbuscules coincides with the accumulation of lipid droplets in the fungal cytoplasm. It has therefore been suggested that the collapse of arbuscules and the associated retraction of cytoplasm to the hyphae is required for the translocation of lipid droplets (Kobae et al., 2014).

Both the formation and the collapse of arbuscules are important checkpoints where the plant can control the symbiosis. The formation of new arbuscules is tightly controlled by the plant to balance the costs of symbiosis with the potential benefits. When the phosphate concentration in the soil is high, the benefits of AM symbiosis are low, and the formation of new arbuscules is inhibited (Kobae et al., 2016). In several plant mutants in which the symbiotic nutrient transfer is disturbed, the arbuscules collapse prematurely (Javot et al., 2007; Baier et al., 2010; Gutjahr et al., 2012; Krajinski et al., 2014; Wang et al., 2014). This shows that the symbiotic functionality of the arbuscule is monitored and required for full maturation. The pre-mature collapse of PHOSPHATE TRANSPORTER 4 (PT4) mutants is suppressed when plants are nitrogen starved (Breuillin-Sessoms et al., 2015). This suggests that plants are able to integrate the requirement and $A M$ contribution of multiple nutrients to decide whether or not AM symbiosis is beneficial. It is unknown whether it is the plant or the fungus that initiates collapse. The plant may reduce its losses by aborting dysfunctional arbuscules. Alternatively, it may reduce the carbon flow to low quality symbionts, triggering the fungus to abort the arbuscules. The latter possibility would make the maintenance of arbuscules a sanctioning mechanisms on a cellular scale, which has been hypothesized to be essential to keep AM symbiosis beneficial during evolution (Kiers et al., 2011; Walder \& Van Der Heijden, 2015). 


\section{Symbiosomes and rhizobium symbiosis}

Most plants from the legume family and the genus Parasponia from the Cannabaceae family can form an endosymbiosis with nitrogen fixing rhizobia. Rhizobia are a paraphyletic group of different nitrogen fixing $\alpha$ - and $\beta$-proteobacteria that convert atmospheric nitrogen into ammonium that can be used by the plant, in exchange for carbohydrates. The rhizobia are hosted in cells of special plant organs called nodules. The rhizobia enter the plant root via infection threads; cell wall bound tubular infection structures that traverse plant cells. At the same time, the nodule primordium is formed. Nodule primordium cells are infected by rhizobia. For most legumes, this involves the release of rhizobia from the infection threads into the cytosol where they remain surrounded by a plant derived peri-bacteroid membrane. After release, the bacteria divide and eventually form nitrogen fixing organelle-like structures called symbiosomes that fill most of the cell. The division and expansion of symbiosomes is associated with a large increase in the total surface of the peri-bacteroid/symbiosome membranes (Robertson \& Lyttleton, 1984). Thus, symbiosome development depends on the continued delivery of vesicles after release. In Parasponia, as well as several basal legume species, the bacteria are never released, but are hosted in branched tubular membranes called fixation threads that remain connected to the infection thread or plasma membrane (Trinick, 1979). In contrast to infection threads, fixation treads do not have a structured cell wall, forming a rhizobial host microbe interface reminiscent of arbuscules. In the legume genus Chamaecrista a continuous range of infection structures from fixation threads to symbiosomes can be found (Sprent, 2009). This shows that although symbiosomes seems at first glance structurally very different from arbuscules, evolutionary these structures may be very related.

Like arbuscules, the symbiosome membrane that forms the rhizobial host-microbe interface is specialized for the exchange of nutrients between the two partners. Since the bacteria in symbiosomes are no longer connected to the extracellular space, all bacterial nutrition must be provided by the plant across the symbiosome membrane. Proteomics approaches in Medicago and soybean revealed the presence of a range of transporters on the symbiosome membrane, such as $A B C$-type oligopeptide transporters, sugar transporters, phosphate transporters, an amino acid permease, a nucleotide transporter, oligopeptide transporters, and a sulphate transporter (Catalano et al., 2004; Krusell et al., 2005; Clarke et al., 2014, 2015). Like on the peri-arbuscular membrane, proton pumps are present that create a proton gradient across the symbiosome membrane that may be used to energize the transporters (Pierre et al., 2013).

Arbuscules play an important role in monitoring the nutrient delivery and controlling the symbiosis by controlling their maintenance. In contrast, rhizobium symbiosis is mainly controlled on the level of whole nodules, while the maintenance of symbiosome symbiosomes seems to play only a marginal role in controlling the symbiosis. Instead 
of terminating individual symbiosomes, legumes control their colonization upstream of symbiosome formation by controlling infection thread formation, the number of nodules that are allowed to form, and the activity of the meristem of nodules. Downstream of symbiosome formation, the symbiosis can be terminated by a process called nodule senescence. During senescence the bacteroids are digested by the plant after which the whole plant cell dies. (Van de Velde et al., 2006). Bacteria that are unable to fix nitrogen can mature into symbiosomes, although the nodules senesce quickly (Hirsch et al., 1983). So even though there appears to be a sanctioning mechanism that terminates ineffective symbionts, this mechanism is distinct from the control of AM symbiosis by arbuscule termination.

\section{Haustoria and biotrophic filamentous pathogens}

The ability to be hosted inside plant cells is not limited to symbiotic microorganisms. Also (hemi-) biotrophic pathogens, in particular filamentous fungi and oomycetes, are capable of invading living plant cells. Biotrophy among filamentous pathogens has evolved multiple times independently (Baxter et al., 2010; Schirawski et al., 2010; Spanu et al., 2010; Duplessis, 2011). Therefore, the intracellular structures formed by pathogens are diverse. Some pathogens like Colletotrichum and Magnaporthe species form biotrophic hyphae in plant cells (O'Connell et al., 1993; Kankanala et al., 2007). Other pathogens form dedicated feeding structures called haustoria (Allen \& Friend, 1983; Knauf et al., 1989; O'Connell \& Panstruga, 2006). Like symbiotic organisms, all parts of the microbe that enter living plant cells remain enclosed by a plant-derived cell membrane. Further, the pathogenic host-microbe interface is devoid of a structured cell wall. Since cooperation of plants in pathogenic host-microbe interface formation is obviously disadvantageous for the plant, the pathogens must rely on pre-existing plant pathways to form a host-microbe interface. In this respect, it has been a long-standing hypothesis that pathogens depend on plant mechanisms involved in symbiotic host-microbe interface formation to forma a pathogenic host-microbe interface.

It is currently unknown whether there are transporters on the peri-haustorial membrane to facilitate transfer of carbon or nutrients to the invading pathogen. Ultrastructural studies on the perihaustorial membrane of Uromyces appendiculatus show that it contains no intramembrane particles, suggesting the membrane is completely devoid of proteins (Knauf et al., 1989). This would mean that the function of haustoria is very different from symbiotic host-microbe interface. Nevertheless, several studies using fluorescent fusion-proteins have shown that some proteins are transported to the peri-haustorial membrane of different pathogens (Wang et al., 2009; Lu et al., 2012). For the hemi-biotrophic pathogen Phytophthora infestans, it has been suggested that it feeds on material that is secreted by the plant after redirection of vacuolar protein degradation pathways towards the haustorium (Bozkurt et al., 2015). Besides its role as feeding structures, the 
pathogenic host-microbe interface is a major site for the secretion of effectors (Whisson et al., 2007; Kleemann et al., 2012; Giraldo et al., 2013; Wang et al., 2017). Many of these effectors are reported to enter the plant cell, where they suppress different defense components or alter host gene expression. However, the translocation of effectors into plant cells is technically challenging to prove, and therefore still slightly controversial (Petre \& Kamoun, 2014).

\section{Evolution of different endosymbioses from AM symbiosis by recruitment of CO/LCO signaling}

In the last decades, it has become clear that there is a common genetic basis underlying both the rhizobial and AM endosymbioses (Bradbury et al., 1991; Kouchi et al., 2010). AM fungi release chitin oligomers (COs) and lipo-chitooligosaccharides (LCOs) that are collectively called Myc-factors (Maillet et al., 2011; Genre et al., 2013; Sun et al., 2015). These signal molecules are perceived by complexes of lysin motif receptor-like kinases (LysM RLKs) on the plasma membrane of plant cells (Maillet et al., 2011; Miyata et al., 2014; Carotenuto et al., 2017). Like AM fungi, rhizobium bacteria also secrete LCOs. LCOs secreted by rhizobia are called Nod factors (Truchet et al., 1991). Although the perception of Nod- and Myc factors likely depends on different complexes of LysM-RLKs (Limpens et al., 2003; Radutoiu et al., 2003; Bozsokia et al., 2017), they feed into a single signaling cascade that is therefore called the common symbiosis signaling pathway (Box1).

The shared use of the common symbiosis signaling pathway shows that rhizobia have co-opted the AM symbiosis dedicated signaling pathway by producing Nod factors that mimic AM LCOs. Activating AM symbiosis related signaling induces symbiotic plant responses that enable rhizobium symbiosis. This recruitment of the common symbiosis signaling pathway is not a unique event in the evolution of endosymbiotic plant microbe interactions. In the non-leguminous plant Parasponia, rhizobium symbiosis has evolved independently from legume-rhizobium symbiosis. Also this symbiosis is dependent on the perception of Nod factors by plant LysM-RLKs and the common sym pathway (Op den Camp et al., 2011; Van Velzen et al., 2017). A different group of nitrogen fixing bacteria from the Frankia genus has established a root nodule symbiosis with plant species from several different families. Also these symbioses require common symbiosis signaling pathway genes (Gherbi et al., 2008; Svistoonoff et al., 2014). Altogether, the different nitrogen-fixing symbioses were hypothesized to have evolved 10 independent times, implying 10 independent recruitments of the AM symbiosis pathway (Doyle, 2011). Alternatively, it has been postulated recently that the different root nodule symbioses have may have a single origin, but has been massively lost in many plant lineages (Van Velzen et al., 2017). Besides nitrogen fixing bacteria, also ericoid- orchid- and ecto-mycorrhizal symbioses seem to have recruited the common symbiosis signaling pathway: Plants of the Ericaceae and Orchidaceae family do not host AM fungi. In most plant species, the loss off AM 
symbiosis leads to a quick loss of symbiosis dedicated genes (Delaux et al., 2014; Bravo et al., 2016), yet the Ericaceae species Rhododendron fortunei and the orchid Phalaenopsis equestrishas both retained common symbiosis signaling genes (Cai et al., 2014; Wei et al., 2016). Although their role has still to be shown experimentally, it is most likely that they have been retained because they have been recruited in ericoid and orchid mycorrhizal symbioses. Finally, ectomycorrhiza do not strictly require the common symbiosis genes and genera like Pinus have lost their common symbiosis signaling genes (Garcia et al., 2015), yet some fungal species do secret LCOs and COs to induce root branching and enhance fungal colonization (V. Puech-Pages, personal communication). The repeated recruitment of the AM dedicated signaling pathway into other symbioses has raised the hypothesis that also biotrophic pathogens may use this pathway to reprogram plants for cooperation in forming haustoria. In chapter 2, I show that the hemi-biotrophic oomycete Phytophthora palmivora is still able to form haustoria in Medicago plants mutated in different common symbiosis genes.

Even though AM- and rhizobium symbiosis share a common symbiosis signalling pathway, the transcriptional reprogramming triggered by rhizobia and AM fungi show a surprisingly low overlap (Hogekamp et al., 2011; Limpens et al., 2013; Nanjareddy et al., 2017). Since the comparison of gene expression induced by different symbionts often relies on the comparison of different tissues, this is in itself perhaps not surprising. However, how the same pathway results in the activation of different core transcription factors remains somewhat puzzling:DELLA and IPD3 are sufficient to activate RAM1 expression. Both DELLA and IPD3 are involved in Nod factor signalling, yet RAM1 is not activated by Nod factors. On the other hand, the expression of auto-active DMI3 or IPD3 is sufficient to induce spontaneous nodule formation in legumes. Both DMI3 and IPD3 are crucial for AM symbiosis, yet AM fungi do not induce nodules on legume plants. Because of this discrepancy in the activation of different symbiotic programs by Nod- and Myc factors, it has been hypothesized that there must be parallel signalling pathways that are required for the specificity of responses triggered by different symbionts (Gutjahr et al., 2008; Oldroyd, 2013; Genre \& Russo, 2016). Despite the limited overlap in AM and rhizobium induced gene expression, there must be some core set of symbiosis genes and processes that are shared between both interaction, which justifies the recruitment of the AM symbiosis pathway into rhizobium symbiosis: First, Among the early responses to both Nod and Myc factors are the expression of extracellular glycoproteins ENOD11 and ENOD12 (Albrecht et al., 1998; Sun et al., 2015). Further, Nod- and Myc factors both induce lateral root branching (Maillet et al., 2011). But biologically the most relevant process that is regulated by the common symbiosis signalling pathway is the formation of a host-microbe interface (Limpens et al., 2005; Morandi et al., 2005; Ovchinnikova et al., 2011). Currently, it is unknown how the common symbiotic signalling regulates host-microbe interface formation, but among the genes that are required is Vapyrin (VPY). VPY expression is triggered by both Myc- and Nod 


\section{BOX 1; CO/LCO signalling in symbiosis}

Schematic overview of the requirement for symbiosis genes in Myc- and Nod factor signalling. Genes in red are involved in both symbioses, and can be viewed as common symbiotic signalling genes. For clarity, only Medicago gene names are shown, although references refer to both Medicago and Lotus studies.

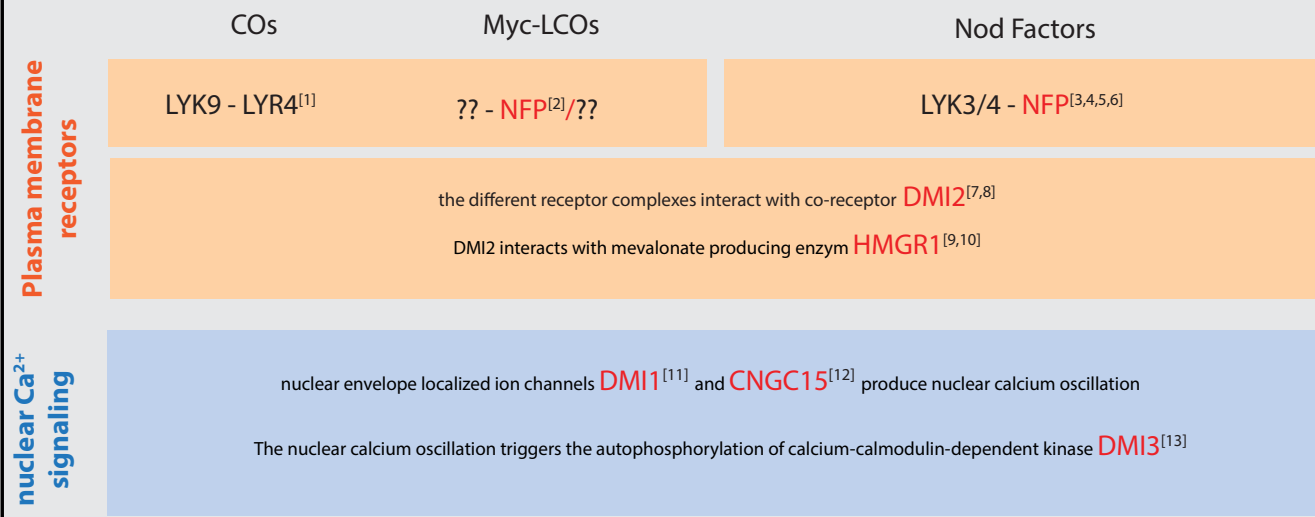

DMI3 binds and phosphorylates transcription factor IPD $3^{[14]}$

IPD3 interacts with DELLA ${ }^{[15,16]}$

DELLA interacts with NSP2 ${ }^{[15,17]}$

IPD3 and DELLA both induce RAM1 expression ${ }^{[18,16]}$

RAM1 induces RAM1 and RAD1 ${ }^{[18]}$

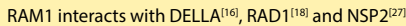

DMI3 is required for pre-penetration aparatus formation ${ }^{[25]}$

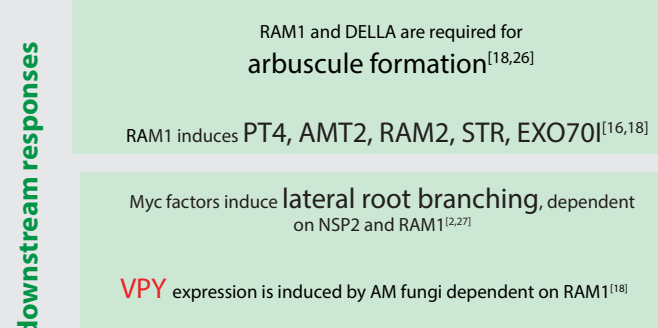

IPD3 and DELLA induce $N I N^{[19]}$ and ERN1 ${ }^{[20,15,17]}$ expression NSP2 interacts with NSP $1^{[21]}$ NIN induces Cytokinin signaling ${ }^{[22]}$

Cytokinin perception induces NIN, NSP1, ERN $1^{[23]}$

NSP1 and NSP2 induce ERN1 expression ${ }^{[24]}$

NIN, ERN1, NSP1, NSP2 and DELLA are required for Infection thread formation ${ }^{[29,30,31,32,15,17]}$

NIN, NSP1, NSP2 and DELLA are required for Nodule formation ${ }^{[30,31,32,15,17]}$

Nod factors induce lateral root branching, dependent on NSP2, but independent of RAM $1^{12,27]}$

VPY is induced by Nod factors partially dependent on NSP1, NSP2, NIN and DELLA, but independent of RAM $1^{[33,17]}$

\footnotetext{
Nod and Myc factors induce ENOD1 1 expression, dependent on NSP1/NSP2 ${ }^{[34]}$
}

References: 1;(Bozsokia et al., 2017) 2;(Maillet et al., 2011) 3;(Limpens et al., 2003) 4;(Radutoiu et al., 2003) 5;(Madsen et al., 2003) 6;(Arrighi et al., 2006) 7;(Endre et al., 2002) 8;(Stracke et al., 2002) 9; (Kevei et al., 2007) 10;(Venkateshwaran et al., 2015) 11;(Charpentier et al., 2008) 12;(Charpentier et al., 2016) 13;(Miller et al., 2013) 14;(Yano et al., 2008) 15;(Jin et al., 2016) 16;(Pimprikar et al., 2016) 17;(Fonouni-Farde et al., 2016) 18;(Park et al., 2015) 19;(Singh et al., 2014) 20; (Cerri et al., 2017) 21;(Hirsch et al., 2009) 22;(Vernié et al., 2015) 23;(Van Zeijl et al., 2015) 24;(Cerri et al., 2012) 25;(Genre et al., 2005) 26;(Floss et al., 2013) 27;(Gobbato et al., 2012) 28;(Xue et al., 2015) 29;(Middleton et al., 2007) 30; (Marsh et al., 2007) 31;(Catoira et al., 2000) 32;(Oldroyd \& Long, 2003) 33;(Murray et al., 2011) 34;(Sun et al., 2015) 35;(Limpens et al., 2005) 36;(Ovchinnikova et al., 2011) 37;(Pumplin et al., 2010) 38; (Kistner et al., 2005) * IPD3 is required for arbuscule formation in Lotus, but not in Medicago. This is likely due to redundancy with a yet unidentified ortholog. 
factors, and is required for the intracellular infection of plants by both rhizobium and AM fungi (Pumplin et al., 2010; Murray et al., 2011).

Summarizing, the rhizobia and probably many other endosymbionts have co-opted AM symbiosis related processes by activating the common symbiosis signalling cascade. Among the processes that are recruited from AM symbiosis is the formation of a host-microbe interface. Thus, it is likely that at least some of the mechanisms for the formation of a symbiotic microbe interface are shared between different symbionts.

\section{Mechanisms of host-microbe interface formation}

The formation of a host-microbe interface presents two major challenges to the host cell: 1). A new membrane compartment has to be created, which requires the expansion of membrane at the exact location where the microbe enters the cell and grows. 2). The proteins that are required for the functionality of the interface membrane such as nutrient transporters have to be delivered specifically to this membrane, and not to any other membrane compartment. This requires the redirection of existing membrane trafficking pathways to the host-microbe interface, or the evolution of a completely new pathway by duplication and neo-functionalization of regulators involved in other vesicle trafficking pathways.

To understand the nature of the vesicle traffic that is (re-)directed to the host-microbe interface, studies have been performed to compare the identity of the different host-microbe interface membranes to existing membrane compartments like the plasma membrane, endosomes, the vacuole or the ER. Symbiosomes were shown to be labeled by the plasma membrane marker SYP132, while they are in later stages also labeled by late endosome/vacuole markers such as Rab7and TIP1g (Limpens et al., 2009; Gavrin et al., 2014). In contrast, arbuscules were shown to accumulate different plasma membrane/ exocytosis markers, but never accumulate endosome markers (Genre et al., 2012; Ivanov \& Harrison, 2014). Haustoria of different pathogens were shown to be labeled by some, but not all tested plasma membrane markers (Lu et al., 2012), accumulate endosome marker Rab5, and in some cases also the late endosome marker Rab7 (Lu et al., 2012; Bozkurt et al., 2015; Inada et al., 2016). The presence of different identity markers on host-microbe interfaces show that interface formation may be dependent on the recruitment of (parts of) multiple vesicle trafficking pathways, which is not necessarily shared between the different plant-microbe interactions, or between different stages of host-microbe interface development. However, the different interactions have in common that exocytosis has been shown to be functionally required (Ivanov et al., 2012; Kim et al., 2014; Zhang et al., 2015b). Therefore, exocytosis may be the main trafficking pathway that contributes to the formation of all three host-microbe interfaces. 
The role of exocytosis in arbuscule and symbiosome formation first became clear from the requirement for exocytosis related vesicle SNARE (v-SNARE) proteins (Ivanov et al., 2012). SNAREs are key regulators of vesicle fusion to specific membrane compartments (Box 2). Ivanov and colleagues have shown that both arbuscule formation and symbiosome requires the presence of two closely related v-SNAREs, VAMP721d and VAMP721e (Ivanov et al., 2012). These genes are embedded within the VAMP72 family, which is involved in vesicle fusion to the plasma membrane. Knockdown of VAMP721d/e blocked the release of rhizobium in nodule cells, as well as the formation of arbuscular branches. In contrast, root growth was not affected. (Ivanov et al., 2012). This suggests that VAMP721d/e are dedicated to the formation of a symbiotic host-microbe interface, and not required for traffic to the plasma membrane. It is not known how these VAMPs relate to the common symbiotic signalling pathway, but it is clear that rhizobium recruited regulators of exocytosis that were dedicated to arbuscule formation to form its own host-microbe interface. In chapter 2, I show that VAMP721d and-e are not required for the formation of haustoria formed by the hemi-biotrophic pathogen Phytophthora palmivora. This indicates that host membrane traffic to the microbe interface is recruited in a different way by this pathogen compared to AM fungi and rhizobia.

The dedication of VAMPs to symbiotic host-microbe interface formation raises the question whether more exocytosis related proteins have been duplicated to create a separate membrane trafficking pathway. I define a separate trafficking pathway as a distinct population of vesicles that contains specific cargo and whose fusion can be regulated independent of other trafficking pathways. The use of separate trafficking pathways for transport to the plasma membrane and the host-microbe interface would be a simple model to allow strict targeting of proteins to each compartment that results in their distinct protein composition. In plants, the use of multiple exocytosis pathways to regulate the trafficking of subsets of proteins has been suggested many times (Rehman et al., 2008; Karnik et al., 2015; Wang et al., 2016; Li et al., 2017). However, these claims are often based on specific mutations or chemical inhibitors of the secretory machinery that have different effects on the delivery of different plasma membrane proteins. Although these are indications for their existence, separate pathways following our strict definition has never been shown. In contrast to the lack of clear evidence for multiple exocytosis pathways in plants, their existence is clear in animals. In mammalian polarized epithelial cells, the traffic to the apical and basolateral plasma membrane domains involves distinct populations of vesicles (Matter \& Mellman, 1994; Mostov et al., 2003; Mellman \& Nelson, 2008). These vesicles are labelled by distinct VAMPs, while the two target membrane domains are labelled by distinct t-SNAREs (Kreitzer et al., 2003; Martinez-Arca et al., 2003; Sharma et al., 2006). 


\section{Chapter 1}

\section{BOX 2; SNARES}

Soluble $\mathrm{N}$-ethylmaleimide-sensitive factor-attachment protein receptors (SNAREs) are proteins that drive membrane fusion. During the fusion of a vesicle to a target membrane, a SNARE protein on the vesicle interacts with a complex of SNAREs on the target membrane. The strong interaction between the SNAREs on the opposing membranes (the formation of a transSNARE complex) provides the energy that is required to draw the two lipid bilayers together (Stein et al., 2009). A SNARE complex always consists of 4 SNARE domains of different classes, $R, Q a, Q b$ and $Q c$. For simplicity, SNAREs are often classified into vesicle-SNAREs ( $v$-SNAREs) that are present on the vesicle and target membrane-SNAREs ( $t$-SNAREs) present on the target membrane. Often, one v-SNAREs protein contributes the R-SNARE domain, while a complex of t-SNAREs proteins contribute the $\mathrm{Qa}, \mathrm{Qb}$ and $\mathrm{Qc}$ domains. After vesicle fusion, both $\mathrm{v}$ - and t-SNAREs are present on the same membrane as a cis-SNARE complex. This complex is dissociated by accessory proteins, after which the v-SNAREs are endocytosed to be reused in or to be degraded.

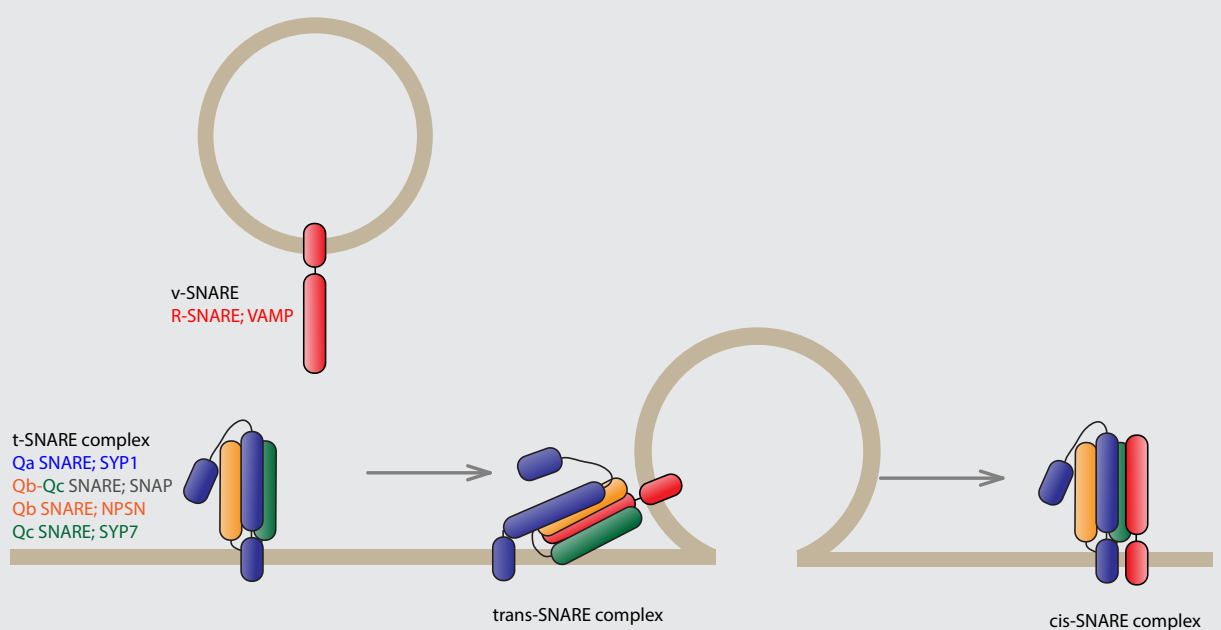

Each vesicle fusion event involving a specific organelle and specific population of vesicles requires different SNAREs. Therefore, the genome of all eukaryotes encodes multiple copies of each class of SNARE proteins. The localization of different SNAREs to different subcellular compartments may be a primary reason for the existence of several SNAREs of one class. Further, since the SNARE proteins involved in vesicle fusion to different organelles do generally not form SNARE complexes (Mcnew et al., 2000), it has been suggested that the specificity between SNARE interactions determines the specificity of vesicle fusion to the right target membrane.

The amount of SNAREs related to exocytosis has in multicellular plants expanded to be encoded by small families (Sanderfoot, 2007). Knockout of individual family members results in different phenotypes related to different processes (Lauber et al., 1997; Collins et al., 2003; Kwon et al., 2008; Ichikawa et al., 2014). This shows that the different family members are used for exocytosis during different biological processes. Within the exocytosis related SNAREs, there seems to be little specificity in SNARE interactions (Chapter 4, table1). Instead, the difference between the individual family members seems to be related to their expression pattern (Enami et al., 2009), dynamics (Nielsen \& Thordal-Christensen, 2012), and interaction with regulatory proteins (Honsbein et al., 2011; Park et al., 2012).

It has been suggested that specific interactions between $\mathrm{v}$ - and t-SNAREs control the specificity of vesicle fusion (Mcnew et al., 2000), making them the key regulators to separate different trafficking pathways. Therefore, we questioned whether symbiotic host-microbe interface formation depends on dedicated t-SNAREs in addition to v-SNAREs. In chapter 3, I describe the functional characterisation of Medicago SYP132 as a t-SNARE that is indispensable for host-microbe interface formation. In most dicot plants SYP132 is spliced into 
two spliceforms; SYP132 $\alpha$ and SYP132 $\beta$. Whereas SYP132 $\beta$ is required for general plant development, SYP132a is specifically required for arbuscule and symbiosome formation. Thus symbiotic host-microbe interface formation depends on dedicated v- and t-SNAREs. Besides these SNAREs, it has been shown that the formation of arbuscules depends on a specific exocyst subunit EXO70l (Zhang et al., 2015b). The exocyst is a protein complex, that is involved in the docking of vesicles before vesicle fusion (Zhang et al., 2010b). In particular EXO70 subunits associate with the target membrane and can define different membrane domains (Sekereš et al., 2017). EXO70I was shown to interact with VPY (Zhang et al., 2015b). Since EXO70l and VPY expression are induced by RAM1 and RAD1 (Park et al., 2015; Xue et al., 2015; Pimprikar et al., 2016), these proteins may be the link between the common symbiotic signalling pathway and the regulation of exocytosis. The dedication of EXO70/ to AM symbiosis is in line in the hypothesis that key regulators of exocytosis have been duplicated to generate a symbiosis specific trafficking pathway, although EXO7Ol is not required for symbiosome formation (Zhang et al., 2015b). Finally, it has been shown that traffic of nodule-specific cysteine rich (NCR) peptides to symbiosomes depends on a nodule-specific signal peptidase DNF1 (Van de Velde et al., 2010; Wang et al., 2010a). It was proposed that proteins can be loaded into a specific trafficking pathway during their production in the ER if they have a specific signal peptide that can function as an address label for symbiosomes (Hohnjec et al., 2009).

If the duplication of regulators of exocytosis truly resulted in the emergence of an alternative trafficking pathway for host-microbe interface formation, then the individual regulators should have neo-functionalized and be different from their non-symbiotic paralogs. However, for neither VAMP721d/e, SYP132a, EXO70I, or DNF1 it has been tested if and how they are different from their orthologs involved in 'default' exocytosis. In chapter 4, I show that VAMP721d/e as well as SYP132a are functionally redundant with their non-symbiotic paralogs. Thus, SNAREs cannot be involved in separating different exocytosis pathways. Further, it has been shown that several proteins can be targeted to either the plasma membrane or the peri-arbuscular membrane, depending on the timing of their expression (Pumplin et al., 2012). Based on this observation, the authors proposed that there is only one exocytosis pathway, which is temporarily redirected to the arbuscule during arbuscule formation. If there are no separate pathways for general exocytosis and host-microbe interface formation, there must be something else that explains the evolution of dedicated SNAREs. In chapter 5, I functionally compare the proteins encoded by the two spliceforms of SYP132 in detail. Although both spliceforms are equally capable of functioning in arbuscule and symbiosome formation, there is a difference in the protein dynamics, and the replacement of SYP132a with SYP132 $\beta$ results in plants with a reduced biomass upon mycorrhization. This suggests that SYP132a has functionally specialized, and is required for full functionality of arbuscules. In summary, my work shows that symbiotic SNAREs are functionally specialized to modify a pre-existing exocytosis pathway, which is required for the full functionality of the host-microbe interface. 
6

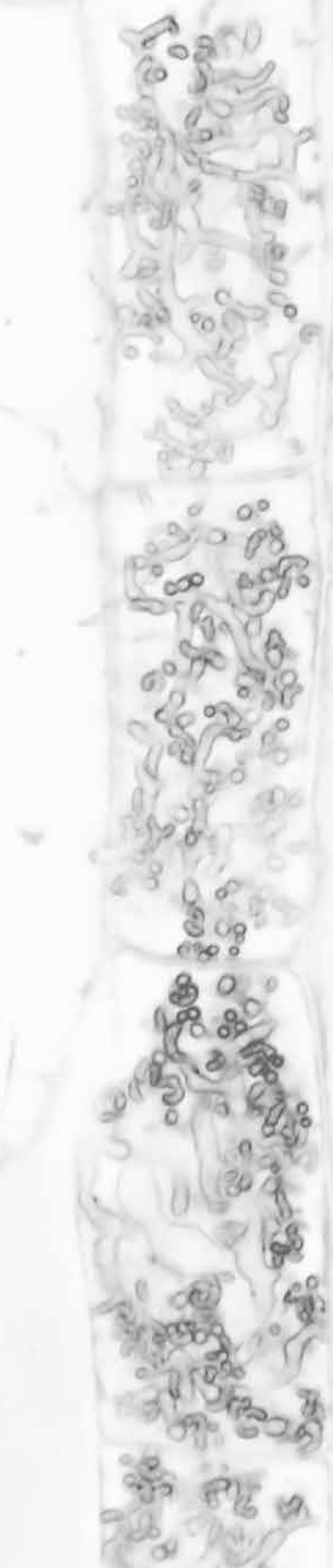




\section{Chapter 2}

\section{Haustorium formation in Medicago truncatula roots infected by Phytophthora palmivora does not involve the common endosymbiotic program shared by AM \\ fungi and rhizobia}

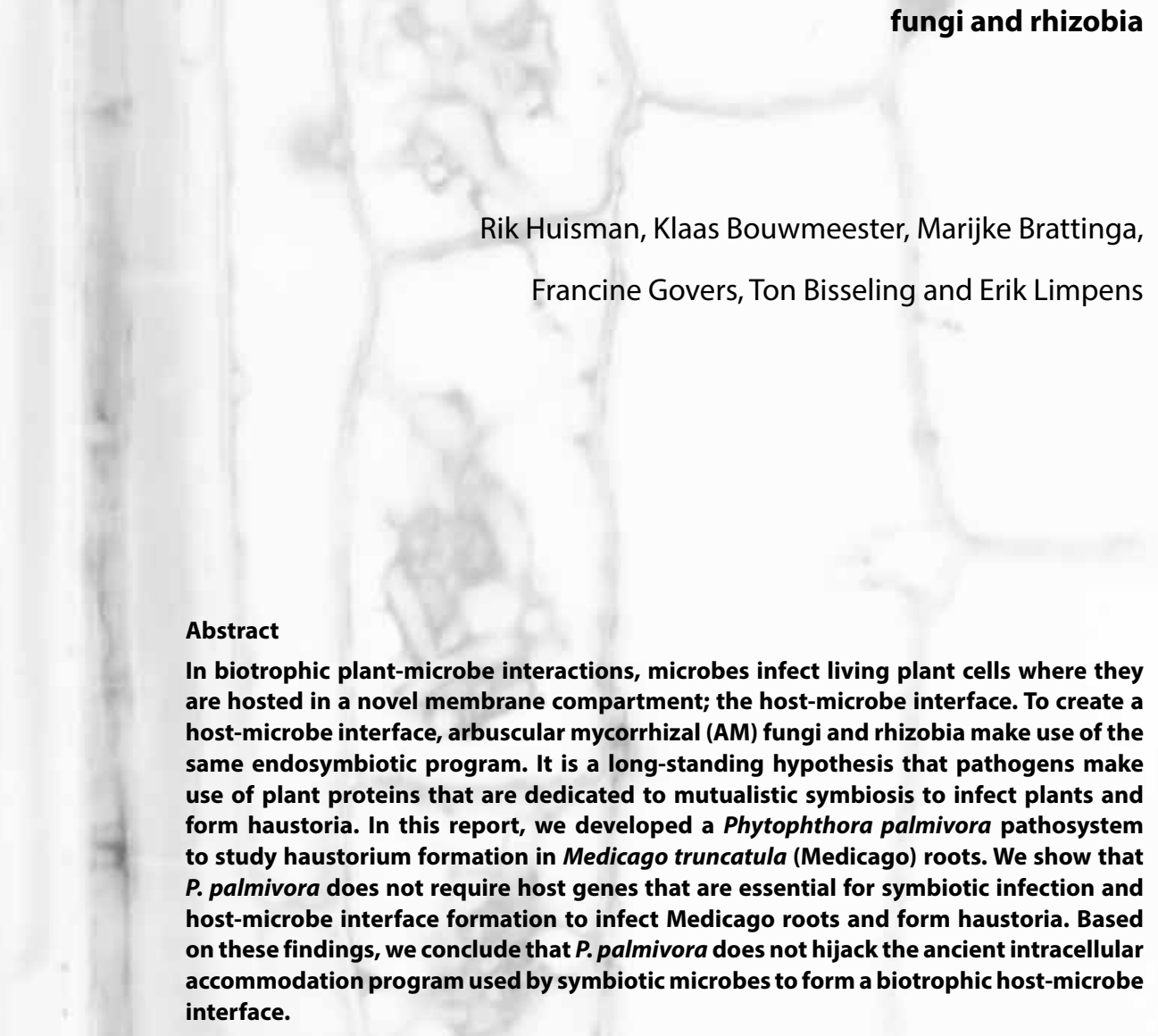

This chapter has been published as:

Huisman, R., Bouwmeester, K., Brattinga, M., Govers, F., Bisseling, T., and Limpens, E. 2015. Haustorium formation in Medicago truncatula roots infected by Phytophthora palmivora does not involve the common endosymbiotic program shared by arbuscular mycorrhizal fungi and rhizobia. Molecular Plant-Microbe Interactions 28:1271-1280. 


\section{INTRODUCTION}

Plants can be colonized by a diversity of biotrophic microbes that invade living plant cells. The relation of these microbes with their host plant varies from mutualistic symbiosis to pathogenesis. A common event in biotrophic interactions is the formation of a host-microbe interface. A host-microbe interface is a specialized membrane compartment in which all or part of the microbe resides within the plant cell. This interface is the fundament of biotrophy, as it facilitates the controlled exchange of nutrients and signals between plant and microbe. Well known examples of host-microbe interfaces are; the peri-arbuscular membrane surrounding arbuscules formed by arbuscular mycorrhiza (AM) fungi, the peri-bacteroid membrane surrounding bacteria in the symbiosis between legumes and rhizobia, and the extrahaustorial membrane surrounding haustoria formed by pathogenic fungi and oomycetes (Parniske, 2000). The intracellular colonization of plant cells, including host-microbe interface formation, requires the active contribution of the plant. Decades of research provided insight into the mechanisms that control the infection and host-microbe interface formation in symbiotic interactions (Harrison, 2012; Oldroyd, 2013). However, how pathogens force plants to cooperate in their intracellular colonization remains an intriguing question.

AM symbiosis is one of the most ancient biotrophic plant-microbe interactions that emerged 450 million years ago (Redecker et al., 2000). Endosymbiosis between plants and nitrogen fixing bacteria, and interactions between plants and biotrophic pathogens evolved multiple times independently, later in evolution (Baxter et al., 2010; Spanu et al., 2010; Doyle, 2011). The genetic constraints for intracellular infection of plant cells are highlighted by the fact that several key components of AM symbiosis have been co-opted in the more recently evolved endosymbiosis between legumes and rhizobia (Summarized in Table 1). Both AM fungi and rhizobium bacteria produce lipochito-oligosaccharide signal molecules (LCOs) that activate a set of common symbiotic signalling (SYM) genes required for intracellular infection (Parniske, 2008; Oldroyd, 2013). Furthermore, AM fungi and rhizobium bacteria share the same exocytosis pathway, marked by two v-SNAREs (VAMP721d/e) to form arbuscules and symbiosomes, respectively (Ivanov et al., 2012). The signalling pathway shared between AM symbiosis and legume-rhizobium symbiosis has been co-opted independently in the interaction between rhizobia and the non-legume genus Parasponia (Op den Camp et al., 2011). Also in the interaction of plants with actinorhizal bacteria, where bacteria are hosted in membrane compartments, common SYM genes are indispensable (Gherbi et al., 2008; Svistoonoff et al., 2014). These data suggest that AM symbiosis provided the blueprint for many endosymbiotic relations that evolved later. This has raised the intriguing hypothesis that many biotrophic microbes including pathogens, make use of a generic program to be accommodated inside plant cells (Parniske, 2000; Rey \& Schornack, 2013). 
This hypothesis is supported by observations that show overlap in pathogenesis and symbiosis: 1). Haustoria and arbuscules are structurally similar, as both are devoid of a structured cell wall, and contain a specialized plant derived membrane, with a unique protein composition (O'Connell \& Panstruga, 2006; Hardham, 2007; Lu et al., 2012; Gutjahr \& Parniske, 2013). 2). Cells that host haustoria undergo endoreduplication, similar to cells that host arbuscules and symbiosomes (Williamson \& Hussey, 1996; Vinardell et al., 2003; Chandran et al., 2010; Bainard et al., 2011). 3). Plant cells often respond to pathogen invasion by aggregating the cytoplasm at the site of contact. In the case of infection of Medicago truncatula (Medicago) by Colletotrichum trifolii and Phoma medicaginis, this aggregation was shown to depend on the common SYM pathway (Genre et al., 2009). 4). Although pathogens are not known to produce LCOs, they may activate common SYM genes in an alternative manner (Liang et al., 2014). Next to LCOs, short chain chitin oligomers were also implicated in symbiotic signalling between AM fungi and Medicago (Genre et al., 2013). In addition, the receptors for perceiving chitin and symbiotic LCOs are related (Nakagawa et al., 2011; De Mita et al., 2014), as well as early transcriptional responses towards mycorrhiza and pathogens (Güimil et al., 2005).

In this study, we addressed the question whether a generic endosymbiosis program, which is used by different symbiotic microbes to establish infection and intracellular accommodation is also used by pathogens to infect Medicago roots and to form haustoria. Therefore, we set-up a suitable pathosystem to study infection and haustorium formation in Medicago roots. We selected the oomycete Phytophthora palmivora as a suitable hemi-biotrophic pathogen that forms haustoria in Medicago roots and monitored disease development and haustorium formation. We inoculated an extensive collection of Medicago mutants that are impaired in plant (intracellular) colonization during $\mathrm{AM}$ and rhizobial symbiosis with P. palmivora. In addition we examined the role of the vesicle SNAREs VAMP721d and VAMP721e that mark a symbiosis specific exocytosis pathway required for intracellular accommodation, for their role in haustorium formation. 


\section{RESULTS}

\section{Infection of Medicago roots by different Phytophthora species}

In order to find a pathogen that forms haustoria in Medicago roots, we focused on Phytophthora species as these are known to invade roots of a broad range of plant species as causal agents of root rot. Furthermore, Phytophthora is able to form haustoria in roots (Wang et al., 2011; Rey \& Schornack, 2013). In an initial screen, we inoculated one week old Medicago seedlings (Jemalong A17) with mycelial plugs of Phytophthora capsici, Phytophthora cinnamomi, Phytophthora medicaginis or Phytophthora palmivora. We analysed root colonization three days post inoculation (dpi), using light microscopy on living roots or sections of plastic-embedded roots. All Phytophthora species were able to infect Medicago roots and haustoria were observed in all interactions (Fig. 1, Fig. 4). Medicago roots infected by $P$. capsici showed most severe disease symptoms, as judged by a strong brown coloration of the roots and a high abundance of intracellular hyphae throughout the root, which likely represents necrotrophic colonization (Fig. 1A). Infection by P. cinnamomi, P. medicaginis and P. palmivora was more subtle, as hyphae were predominantly present in the intercellular spaces of the outer cell layers of the root (Fig. 1B and C, Fig. 4). We selected $P$. palmivora for further studies, as transgenic lines expressing GFP or $\beta$-glucuronidase (GUS) (van West et al., 1999; Vijn \& Govers, 2003) were available to facilitate imaging of infection events.
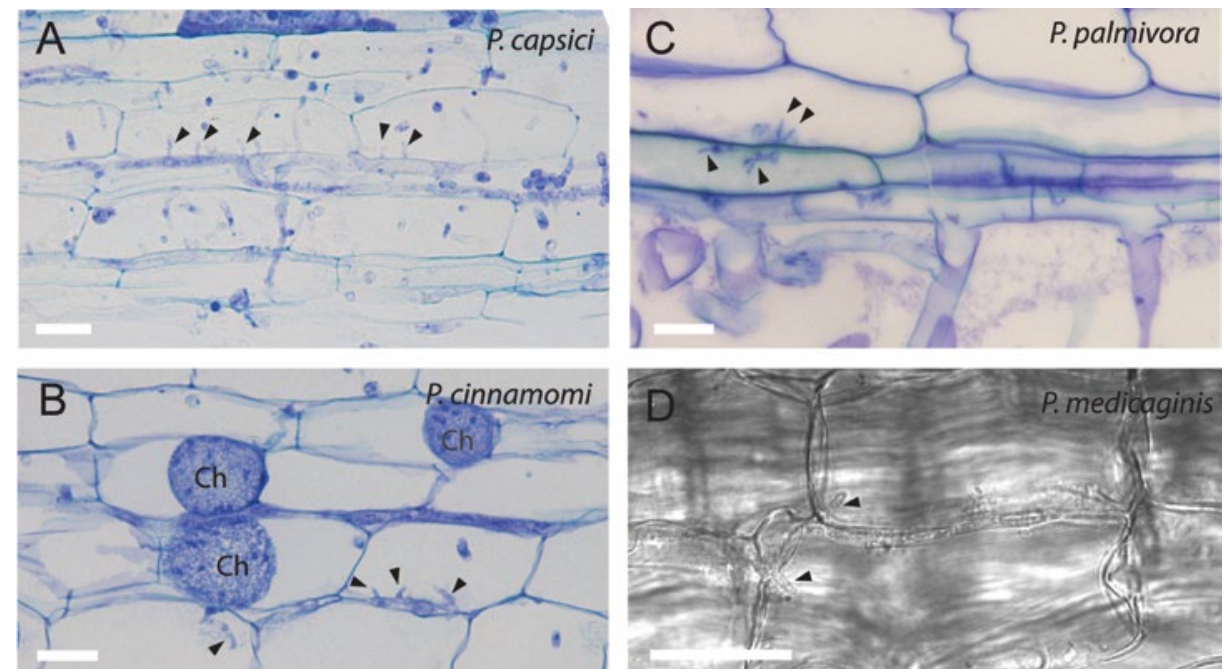

Figure 1. Infection of Medicago roots by different Phytophthora species

\footnotetext{
A, Toluidine Blue stained plastic section $(6 \mu \mathrm{m})$ of Phytophthora capsici infected Medicago root. Haustoria are marked by black arrowheads. B, Toluidine Blue stained plastic section of Phytophthora cinnamomi infected Medicago root. Haustoria are marked by black arrowheads. Chlamydospores (Ch) are forming in plant cells. C, Toluidine Blue stained plastic section (6 $\mu \mathrm{m})$ of Phytophthora palmivora infected Medicago root. Haustoria are marked by black arrowheads. D, Light microscopy image of a hand section through a Phytophthora medicaginis infected root. Outlines of hyphae between cells are visible, as well as haustoria entering cells (black arrowheads). Scale bars represent $25 \mu \mathrm{m}$.
} 


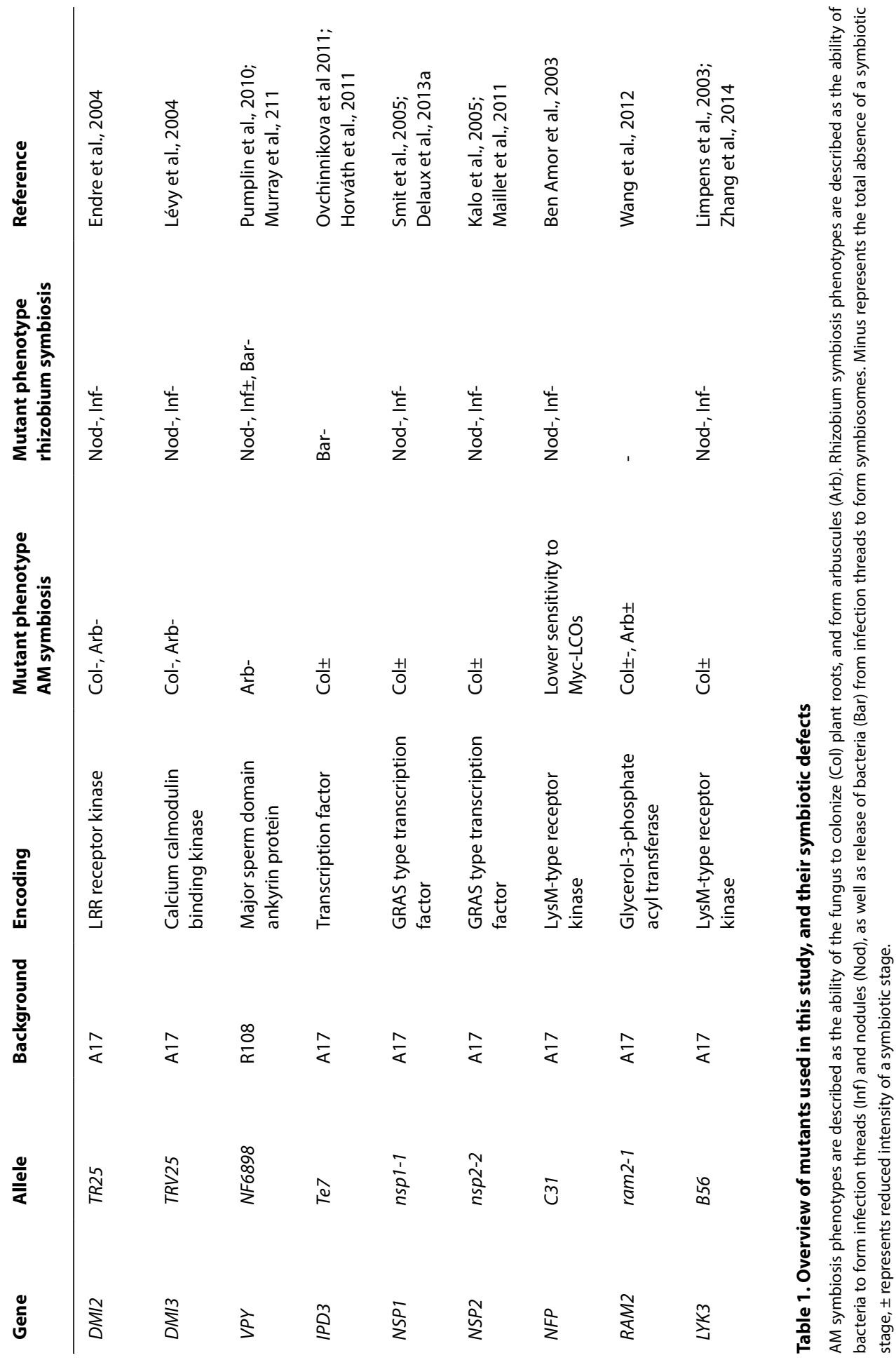




\section{Knockout of Medicago genes required for symbiotic infection does not affect susceptibility towards $\boldsymbol{P}$. palmivora}

To study whether Phytophthora makes use of genes required for symbiotic root colonization, we studied the susceptibility of the Medicago mutants listed in Table 1 towards P. palmivora. We quantified the spread of infection through Medicago roots after point inoculation, similar to the quantification of lesion length as used by Yang and colleagues (Yang et al., 2013). Medicago seedlings were inoculated using a mycelial plug of a GUS-expressing P. palmivora transformant. At $4 \mathrm{dpi}$, GUS staining was used to visualise the spread of hyphae through the roots. We measured the length of the region of the root that was colonized and performed a Mann-Whitney $U$ test to determine the significance of differences between genetic backgrounds. All mutants were in the Jemalong A17 genetic background, except for the VAPYRIN mutant (vpy), which is in the R108 genetic background (Table 1). No significant differences were observed between any of the mutants and their respective wild type control (Fig. 2, Table S2). Notably, the spread of hyphae in the R108 background was significantly more extensive $(p<0.001)$ compared to roots in the $\mathrm{A} 17$ background. This indicates that R108 is more susceptible to P. palmivora infection, than A17.

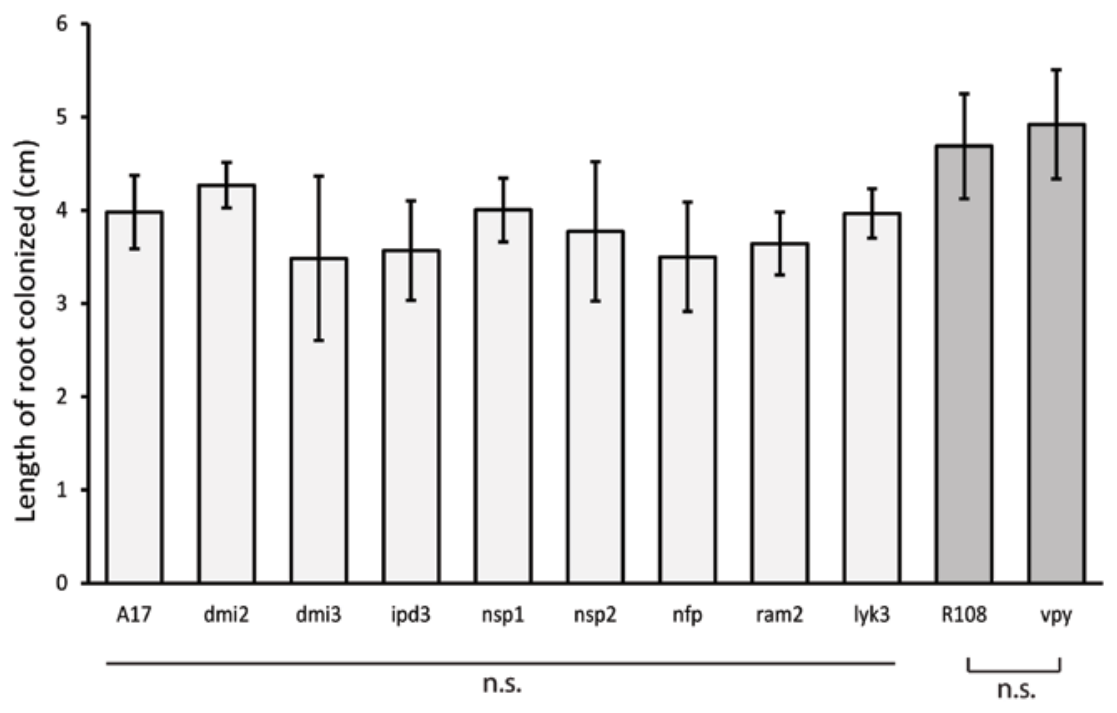

Figure 2. Mutation of symbiosis genes does not affect susceptibility of Medicago roots towards $P$. palmivora

Spread of infection in Medicago mutants after infection with mycelial plugs of P. palmivora GUS, 4 dpi. The length of the region stained by GUS was measured. N.s. indicates non-significant difference in colonization between mutants and their wild type background. $(n=4, p<0.05)$. 


\section{Phytophthora palmivora zoospore inoculation assay}

Analyses of the infection events after inoculation of wild type plants using the mycelial plugs showed that the zone in which haustoria were distinguishable was relatively small, likely due to the point source inoculation. Furthermore, many infected cells along the root contained intracellular hyphae that traversed the cells, which hampered the identification of haustoria. To facilitate more extensive and synchronized haustorium formation along a broader root region, and to obtain a less severe infection, we next inoculated Medicago roots with Phytophthora zoospores in a hydroculture system. One-week-old Medicago seedlings were placed in hydroculture by inserting their hypocotyl in plastic foam floating on demineralized water in polystyrene tubes (Fig. 3).

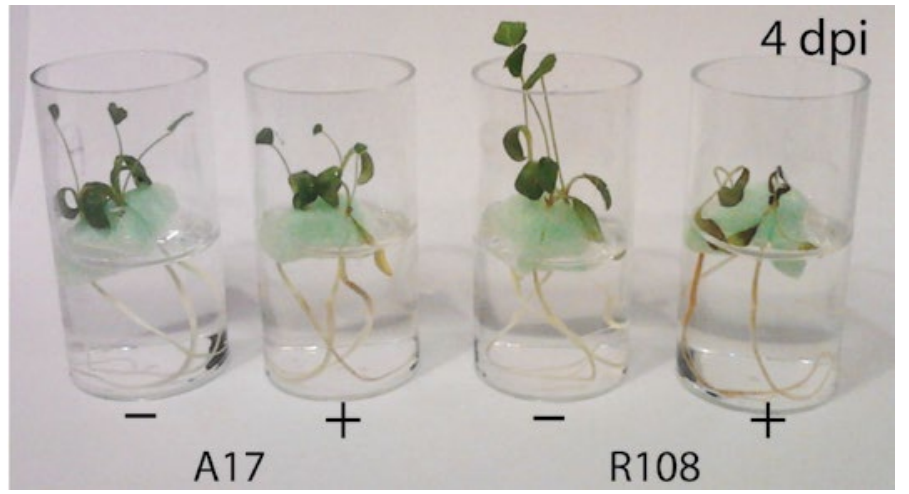

Figure 3. Experimental setup for zoospore inoculation of Medicago seedlings by P. palmivora

Seven-day-old Medicago A17 and R108 seedlings are grown in hydroculture tubes $\varnothing=3 \mathrm{~cm}$. Phytophthora palmivora inoculated plants (+) and mock inoculated plants (-) are shown at 4dpi.

Plants were inoculated by adding 104 GFP- or GUS-expressing P. palmivora zoospores to the water. First we analysed the infection process in the wild type Medicago backgrounds A17 and R108. At one dpi, zoospores had germinated and hyphae had penetrated the epidermis (Fig. 4A). This experimental set-up resulted in an equal spread of infection events over the entire root length with a slight bias toward the root tip (data not shown). $P$. palmivora generally entered Medicago roots by crossing the cells of the epidermis, although it occasionally also penetrated between epidermal cells. Germ tubes entered atrichoblasts as well as root hairs. The hyphae that entered root hairs did not progress in a specific direction, which mostly resulted in aborted infections. Infected epidermal cells were also often heavily colonized with a high abundance of hyphae within a cell. From this primary site of infection, hyphae predominantly progressed parallel to the root surface through the intercellular spaces. At $2 \mathrm{dpi}$, colonization of the root was mainly restricted to the epidermis and outer cortex layer (Figs. 4B and C). Haustoria could be observed in both cell layers. The shape of haustoria varied from digit-like to branched. From $2 \mathrm{dpi}$ onwards, a slight brown coloration of the roots could be observed, which was more pronounced in ecotype R108 compared to A17 (Fig. 3). The brown coloration, which likely represents 
necrotic cells (Yang et al., 2013; Rey et al., 2015), gradually increased in intensity over the course of infection. At $4 \mathrm{dpi}$, the infection had progressed approximately one cell layer deeper into the root with hyphae present between the two outermost cortex layers, whereas at $6 \mathrm{dpi}$, some variation in the amount of infected cell layers was present. In the roots of $A 17$, the hyphae remained mainly restricted to the outer cell layers of the root, showing that inward progression of hyphae had halted (Fig. 4D). Further, the shoots showed no macroscopic symptoms (Fig. 3). In contrast, P. palmivora progressed deeper into roots of $\mathrm{R} 108$ compared to $A 17$, with hyphae present between all cortical cell layers, and invading the vascular bundle (Fig. 4E). Moreover, the shoots of R108 showed severe macroscopic symptoms; at $4 \mathrm{dpi}$, all shoots of R108 had wilted, compared to one shoot in A17 (Fig 3; $n=15$ ). On all roots, new zoosporangia were formed, allowing P. palmivora to complete its lifecycle (Fig. 4F).
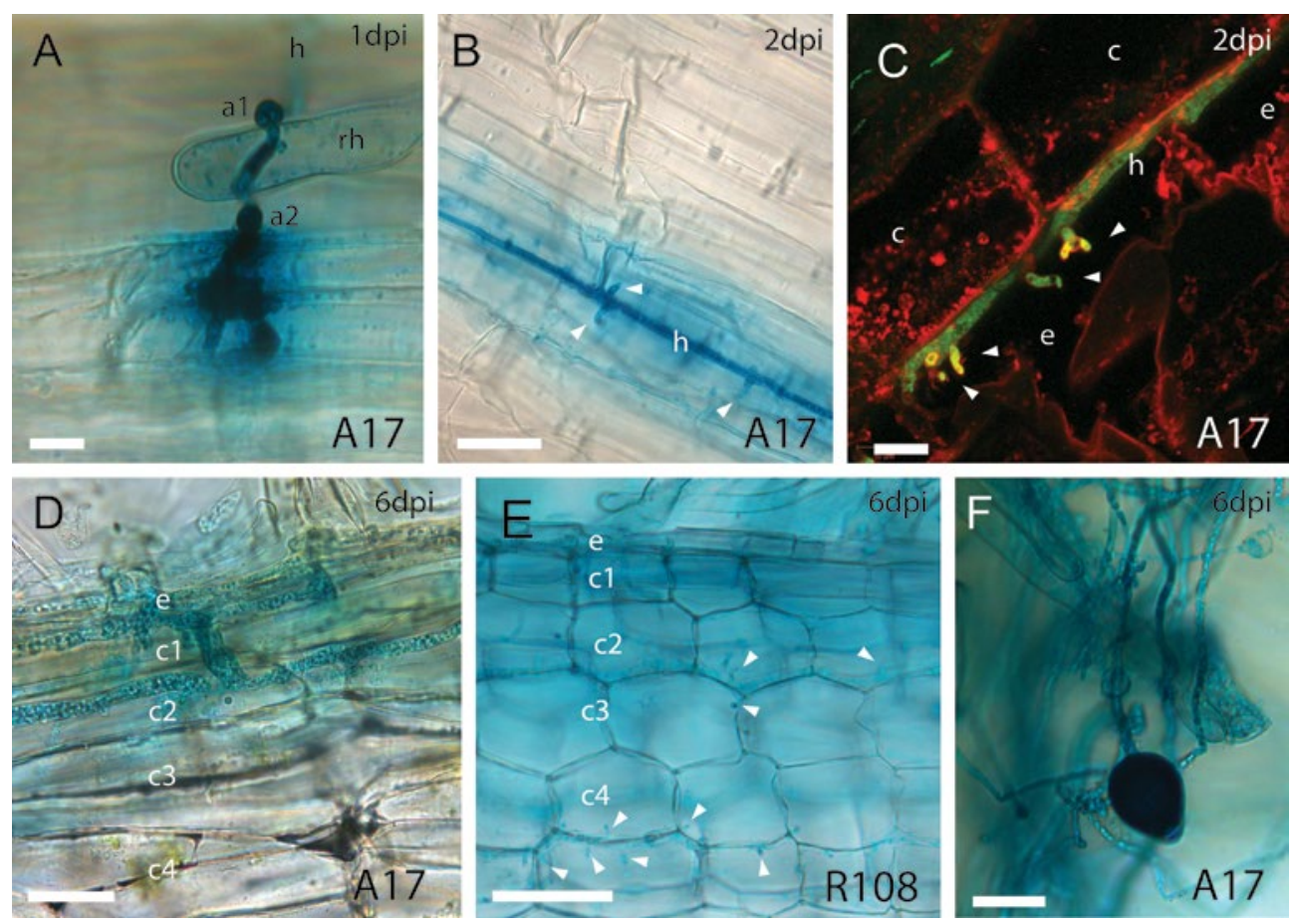

Figure 4. Infection of Medicago roots by $P$. palmivora

A, Z-projection of 3 images showing early infection of a Medicago root by P. palmivora GUS, 17 hpi. P. palmivora structures stain dark blue. A hypha (h) arriving at the root forming an initial appressorium (a1) on a root hair (rh), after which it continues growth towards the root. On a root epidermal cell a second appressorium (a2) is formed, of which subsequently hyphae penetrate the epidermal cells. Scale bar $=25 \mu \mathrm{m}$. B, Hypha (h) growing in the intercellular space in longitudinal direction of the root, $2 \mathrm{dpi}$. Haustoria (white arrowheads) are visible. scale bar $=25 \mu \mathrm{m} \mathrm{C}$, Projection of confocal image stack, 2dpi. P. palmivora GFP infects Medicago, counterstained with FM4-64. A hypha grows in the intercellular space between epidermal cells (e) and cortex cells (c). Haustoria (white arrowheads) are formed in the epidermal cells. FM4-64 staining around the haustoria shows that the haustoria are surrounded by membrane. Scale bar $=10 \mu \mathrm{m}$. D and E, Hand sections through a Medicago A17 (D) or R108 (E) roots, $6 \mathrm{dpi}$ with P. palmivora GUS. Epidermis (e) and different cortex layers (c1-c4) are visible. Haustoria are marked with white arrowheads. Scale bars are $25 \mu \mathrm{m}$. F, Infection $6 \mathrm{dpi}$, showing a zoosporangium. Scale bar $=25 \mu \mathrm{m}$. 


\section{Common symbiotic signalling genes are not required for haustorium formation}

We used the hydroculture system to study whether any of the symbiotic mutants (Table 1) would be impaired in haustorium formation. To facilitate the visualization of haustoria we mainly made used of the GUS-expressing P. palmivora strain. At 2, 4 and 6 dpi, eight roots were harvested and screened for the presence of haustoria. Haustoria were consistently observed in all mutants, in all roots ( $n=24$; Fig. 5, Figure S1), over two repetitions of the experiment. Furthermore, at 6 dpi new zoosporangia were produced on all plant roots, showing that $P$. palmivora is able to complete its lifecycle on all mutants. No obvious differences with respect to root colonization length, intracellular hyphae and depth of hyphal penetration, were observed between the mutants and their corresponding wild type genetic backgrounds.
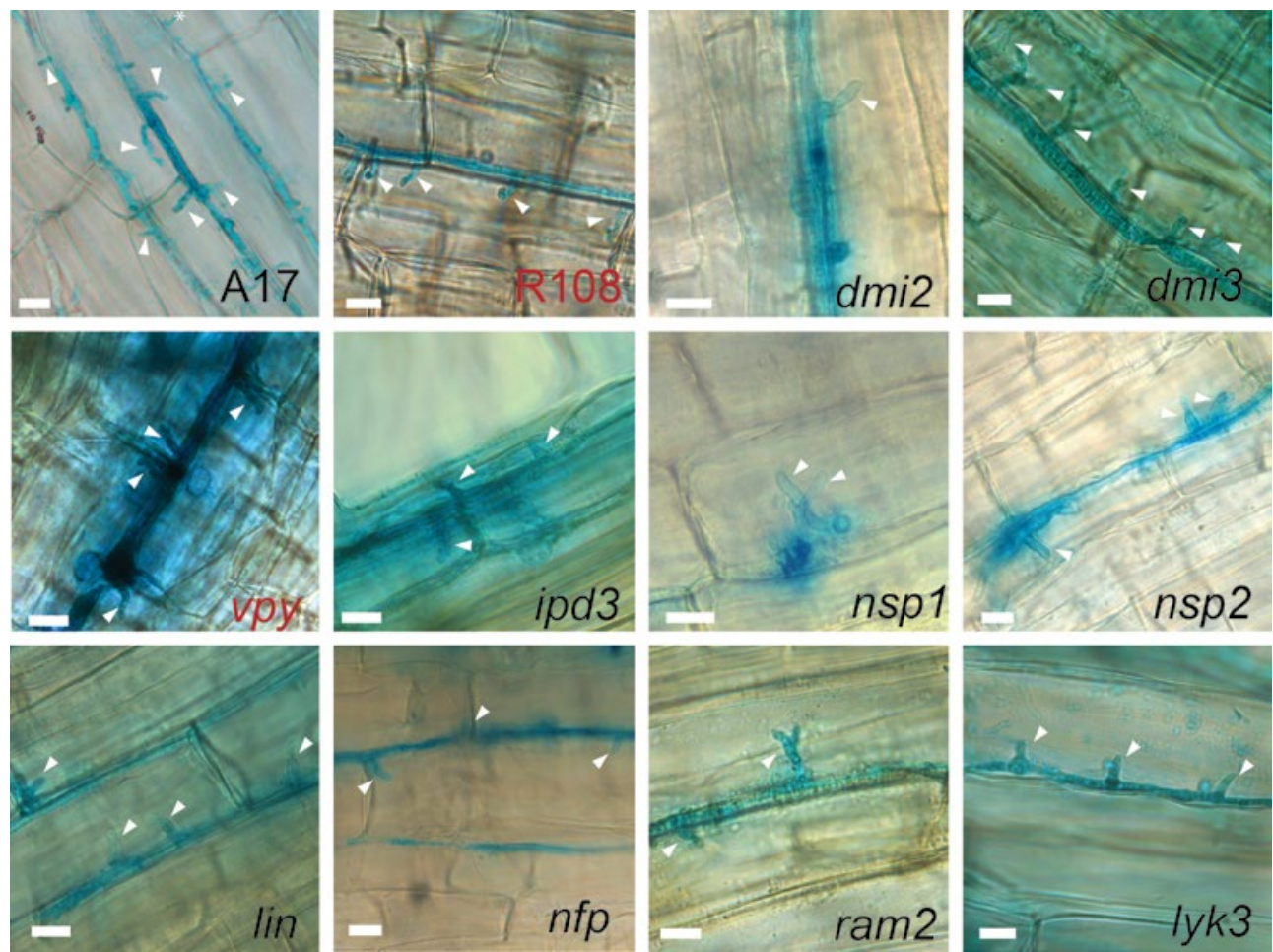

Figure 5. Haustorium formation in roots of Medicago wild type plants and symbiosis mutants

Haustoria (white arrowheads) formed in roots of Medicago ecotypes A17 and R108 and different Medicago mutants, 2dpi. N=24, scale bars represent $10 \mu \mathrm{m}$. 
Of the ten mutants used in this study, only the ram2 mutant was previously tested for its ability to accommodate $P$. palmivora. In that study, it was shown that $P$. palmivora is not able to form appressoria on ram 2 roots, severely reducing the susceptibility of this mutant plant (Wang et al., 2012). In our hands zoospore inoculation resulted in colonisation of the ram 2 mutant and no differences were observed between ram2 and the wild type A17. This is in line with the similar susceptibility of ram 2 mutants and wild type plants to $P$. palmivora after inoculation with mycelial plugs (Fig. 2). We observed normal appressorium formation on the surface of the ram 2 mutant in the hydroculture system. To rule out differences between the strains of $P$. palmivora that were used in our study and that of Wang et al. (2012), we also analysed the infection of A17 and ram2 with the P. palmivora strain 16830 (Wang et al., 2012). Also with this strain appressoria were formed on all ram2 roots like on wild type, resulting in a successful infection and haustorium formation (Fig 6; $n=10$ ). On both ram2 and wild type roots the length of the germ tube connecting cyst and appressorium was in the same range, indicating that $P$. palmivora had no difficulty in forming appressoria (Fig. 6A and B).
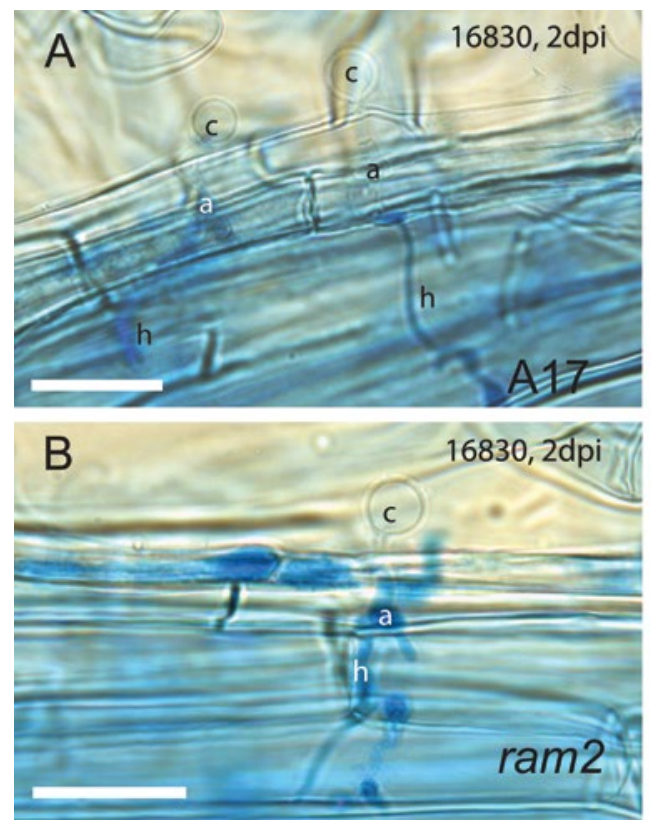

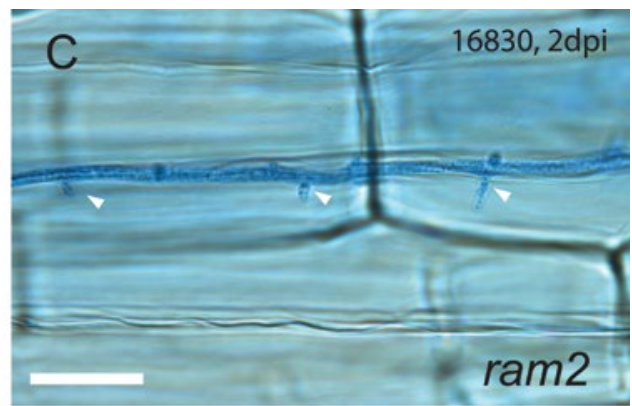

Figure 6. Medicago ram2 mutant plants are infected by $P$. palmivora similar to wild type plants Trypan Blue stained roots showing the infection of $A 17$ (A) and ram 2 mutants ( $B$ and $C$ ) at 2 dpi with P. palmivora isolate 16830. Germinating cysts (c) with short germtubes forming an appressorium (a) from which hyphae (h) penetrate the root. C, In the roots, haustoria (white arrowheads) are formed (C). Scale bars are $25 \mu \mathrm{m}$.

Together, our observations show that in Medicago, the Nod factor receptors, the common symbiosis signalling genes and downstream components are not essential for the formation of a pathogenic host- microbe interface by P. palmivora. 


\section{Knockdown of the symbiosis-specific V-SNAREs VAMP721d and VAMP721e does not affect haustorium formation.}

Since the link between symbiotic signalling and symbiotic membrane traffic has not been resolved, it is possible that these two mechanisms are independently recruited by microbes. Therefore, although biotrophic pathogens do not require symbiotic signalling genes for haustorium formation, it is still possible that they do require the symbiotic membrane trafficking pathway marked by specific v-SNARE proteins. To determine whether these SNAREs are also required for haustorium formation, we tested the ability of $P$. palmivora to form haustoria in axenic root cultures in which these SNARE genes were silenced. Agrobacterium rhizogenes transformed roots were generated using the VAMP721d/e silencing construct described by Ivanov et al. (2012). Several root cultures were produced and VAMP721d/e transcript levels were determined by qRT-PCR. Efficient silencing of VAMP721d and VAMP721e was observed, with transcript levels down to $6 \%$ and $9 \%$ respectively, in root culture \#1 (Fig. 7A). This level of silencing completely blocked the ability of the AM fungus Rhizophagus irregularis to form arbuscules. Intraradical hyphae and arbuscule trunks were observed in VAMP721d/e-RNAi root cultures (Fig. S2A), but arbuscule fine-branches did not form in analogy to the phenotype published by Ivanov et al. (2012). In contrast, empty vector control root cultures did show normal arbuscule formation (Fig. S2B). Two days after inoculation with P. palmivora zoospores we observed haustoria in both the VAMP721d/e-RNAi root culture (Fig. 7C) as well as in the empty vector root culture (Fig 7B). This strongly indicates that haustorium formation by $P$. palmivora does not require the symbiotic v-SNAREs.

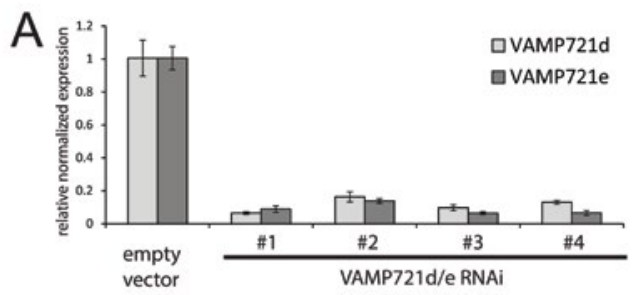

Figure 7. Haustorium formation in VAMP721d/e silenced root cultures

A, Expression levels of VAMP721d and VAMP721e in different VAMP721d/e silenced root cultures relative to the empty vector control. Expression levels were normalized using MtActin2 and MtUbiquitin 10 as reference genes. B and C, Microscopy images of hand sections of a P. palmivora GUS infected root cultures, 2 dpi. Haustoria (white arrowheads) were visible in root cultures transformed with an empty vector (B) as well as in VAMP721d/e silenced cultures (C). Scale bars represent $25 \mu \mathrm{m}$.

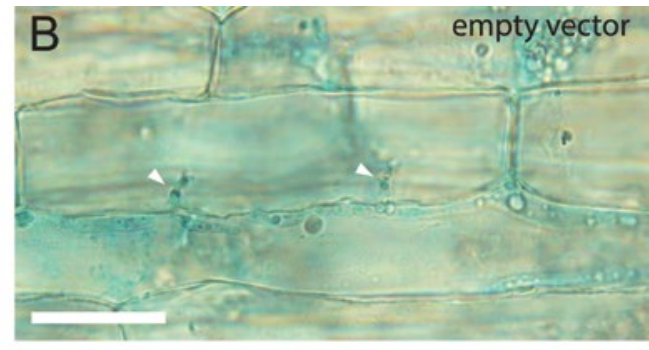

VAMP721d/e RNAi 


\section{DISCUSSION}

In this study, we have developed a pathosystem to study the formation of haustoria in the model legume Medicago truncatula. The relatively long biotrophic phase of Phytophthora palmivora together with its ability to form haustoria in root cells of Medicago allowed us to compare the formation of haustoria with symbiotic host-microbe interfaces in the same plant tissue. We made use of the extensive collection of Medicago mutants to study the role of core symbiosis genes that are shared between AM symbiosis and rhizobium symbiosis in plant-pathogen interactions. The susceptibility towards P. palmivora was not significantly different in any of the tested Medicago symbiotic mutants, showing that these genes are not required for full virulence. We did however observe a higher susceptibility of the wild type ecotype 108 compared to Jemalong A17, in agreement with the data from Samac and co-workers (Samac et al., 2011), which showed more severe disease symptoms on R108 compared to A17 in plants infected with the powdery mildew Erysiphe pisi and Phytophthora medicaginis.

The absence of differences in susceptibility to $P$. palmivora is in contrast with several earlier reports, which show that mutations in symbiosis genes can alter plant defence or pathogen entry. Most remarkable is the discrepancy between our results and those of Wang et al., (2012) and Rey et al. (2015) in relation to the susceptibility of the ram2 mutant towards $P$. palmivora. They reported that $P$. palmivora is unable to form appressoria on ram 2 roots, which are impaired in cutin biosynthesis, resulting in a severely reduced colonization (Wang et al., 2012). Similarly, colonisation by the oomycete Aphanomyces euteiches was reported to be impaired in ram2 roots (Gobbato et al., 2013). However, in our assays $P$. palmivora formed appressoria on ram 2 mutant roots like it did on wild type roots. Furthermore, no differences in root colonization or the ability to form haustoria were observed between ram 2 and wild type roots. Similar results were obtained with the P. palmivora strain originally used by Wang and colleagues. These differences might be explained by different growth conditions in the various studies, although both plate inoculations as well as inoculation in hydroculture did not show any defect in our hands. This strongly suggests that the involvement of cutin as a cue for appressorium formation is not as strict as previously claimed.

The Medicago Nod factor receptor $n f p$ mutant was previously reported to have an increased susceptibility towards the oomycete $A$. euteiches and the fungus C. trifolii (Rey et al., 2013). The increased susceptibility of the $n f p$ mutant to A. euteiches and $C$. trifolii suggests that chitosaccharides released by these pathogens are detected by NFP, which contributes to plant defence (Nars et al., 2013; Rey et al., 2013). In our experiments, infection of the $n f p$ (C31/nfp-1) knock-out mutant by P. palmivora was unaltered compared to wild type. Also, in the recent study by Rey et al. (2015) P. palmivora infection was only marginally affected in the $n f p-1$ mutant. This may suggest that (hemi-)biotrophic pathogens differ in the extent 
to which they elicit defence through NFP. Notably, the observed difference correlates with the presence of chitosaccharides in the cell walls of C. trifolii, and A. euteiches, and the presumed absence of chitosaccharides in Phytophthora species (Meijer et al., 2006; Badreddine et al., 2008; Nars et al., 2013).

Our results show that in the roots, the common SYM genes are not required for haustorium formation or intracellular infection. Thus, in contrast to rhizobia, P. palmivora does not make use of the ancient AM symbiosis program to be hosted inside plant cells. The latter is also in line with the ability of the rice blast fungus Magnaporthe oryzae to intracellularly colonize rice roots mutated in the common SYM gene CCAMK/DMI3 (Marcel et al., 2010). Furthermore, Phytophthora parasitica has been shown to form haustoria in the roots and leaves of Arabidopsis thaliana, which does not engage in AM symbiosis and consequently has lost most genes required for AM symbiosis during evolution (Wang et al., 2011; Delaux et al., 2013b). Similar observations are made in leaves of Lotus japonicus where mutations in common SYM genes do not affect haustorium formation by rust fungus Uromyces loti (Mellersh \& Parniske, 2006). However, it is unclear whether the common SYM genes are active in leaves.

In addition to the involvement of the symbiotic signalling genes, regulation of vesicle trafficking to form a host-microbe interface also appears to be different between P. palmivora and symbionts. In case of arbuscules (or symbiosomes) the formation of the interface requires a specific exocytosis pathway marked by the vesicle SNAREs VAMP721d and VAMP721e (Ivanov et al., 2012). Although we did not completely knock out VAMP721d/e and cannot rule out the contribution of the residual transcript levels to haustorium formation, knockdown of both VAMPs to levels that impair arbuscule formation, did not affect the ability of $P$. palmivora to form haustoria. Like arbuscules and symbiosomes, haustoria do have a specialized plant-derived interface membrane (the extrahaustorial membrane) that is distinguishable from the plasma membrane (Lu et al., 2012). If the formation of the extrahaustorial membrane indeed depends on a specialized membrane trafficking pathway directed towards the haustorium, it could rely on other vesicle SNAREs than those required for symbiotic vesicle traffic. The involvement of different membrane trafficking pathways to accommodate symbionts or pathogens may reflect a different requirement to deliver cell-wall degrading/loosening enzymes by the plant to allow the entry of the microbes. The most significant cell wall remodelling occurs at the switch from arbuscule trunk (which is still cell wall bound) to the fine branches that form the actual symbiotic interface, which are devoid of a structured cell wall (Balestrini \& Bonfante, 2014). Arbuscular mycorrhizal fungi do not have enzymes to degrade plant cell walls and like rhizobium bacteria rely heavily on the host plant to degrade the cell wall to allow the formation of a symbiotic interface (Tisserant et al., 2013). Pathogenic microbes on the other hand often do have the capacity to break-down the cell wall and might therefore be less dependent on the host plant. 
In conclusion, we show that P. palmivora does not hijack the ancient intracellular accommodation program used by symbiotic microbes to form a biotrophic host-microbe interface. Since symbiotic signalling and defence signalling are partially overlapping (Liang et al., 2014), it is likely that the susceptibility of symbiotic signalling mutants to various pathogens will show species-specific differences. However, we have shown that the presence of the symbiotic intracellular accommodation program is not a general prerequisite for the intracellular infection and haustorium formation by pathogens. 


\section{MATERIALS AND METHODS}

\section{Plant growth conditions}

Except for ram 2 mutant seeds, Medicago truncatula seeds were scarified in $96 \% \mathrm{H}_{2} \mathrm{SO}_{4}$ for 10 minutes. Ram 2 seeds were scarified with sandpaper. All seeds were washed in demiwater and subsequently surface-sterilized in $4 \% \mathrm{NaClO}$. After sterilization, the seeds were washed in sterile demi-water, incubated for 1 day at $4{ }^{\circ} \mathrm{C}$ and pre-germinated on Farhaeus plates (Limpens et al., 2004) for 1 day at room temperature in the dark. Seedlings were transferred to Farhaeus plates and grown for 7 days at $25^{\circ} \mathrm{C}$ at a $16 / 8$ hour light/dark regime until inoculation. The Medicago mutants used in this study are listed in Table 1 . The mutations in all mutant lines were verified by sequence analysis.

\section{Medicago transformation}

Medicago A17 plants were transformed using Agrobacterium rhizogenes MSU440, carrying RNA silencing constructs targeting MtVAMP721d and MtVAMP721e according to Ivanov et al. (2012). Transformed roots were transferred to solid M-medium (Bécard \& Fortin, 1988) containing $300 \mu \mathrm{g} / \mathrm{ml}$ Cefotaxime, and incubated at $25^{\circ} \mathrm{C}$ in the dark, until a growing root culture was obtained. The level of VAMP721d/e silencing was determined by qRT-PCR, after which the root culture with the lowest VAMP721d/e transcript levels was selected and maintained on solid M-medium until inoculation.

\section{RNA isolation and qRT-PCR}

RNA was isolated from root cultures with a plant RNA mini kit (EZNA), according to manufacturer's instructions. One microgram of RNA was used for cDNA synthesis using the iScript cDNA synthesis kit (Bio-Rad) according to manufacturer's instructions. Equal amounts of CDNA were used for qPCR using SYBR green supermix (Bio-Rad) in a Bio-Rad CFX connect real-time system qPCR machine. Gene expression levels were determined using gene specific primers listed in supplemental table S1 and cDNA levels were normalized using Actin2 and Ubiquitin10 as reference genes, using the delta-delta Ct method (Livak \& Schmittgen, 2001).

\section{Phytophthora strains and culture conditions}

We made use of Phytophthora palmivora isolate 6370 carrying a GUS gene regulated by the constitutive Bremia lactucae pHam34 promoter (Vijn \& Govers, 2003), Phytophthora palmivora strain 6370 carrying GFP (van West et al., 1999), Phytophthora palmivora isolate 16830 (Wang et al., 2012), Phytophthora capsici stain LT3241 (Wang et al., 2013), Phytophthora cinnamomi Pe-90 (Serrano et al., 2012), and Phytophthora medicaginis strains CBS119902 and CBS117685 (obtained from the CBS-KNAW culture collection) All Phytophthora species, were maintained at $25^{\circ} \mathrm{C}$ on V8 agar plates ( $20 \%$ V8 vegetable juice 
(Campbell's) 3.5g/ $\mathrm{L} \mathrm{CaCO}_{3,} 15 \mathrm{~g} / \mathrm{l}$ micro agar (Duchefa). V8 medium for culturing transgenic lines was supplemented with $100 \mu \mathrm{g} / \mathrm{ml}$ ampicillin, $10 \mu \mathrm{g} / \mathrm{ml}$ amphotericin $B, 20 \mu \mathrm{g} /$ $\mathrm{ml}$ vancomycin and $20 \mu \mathrm{g} / \mathrm{ml}$ geneticin.

\section{Phytophthora palmivora zoospore production}

For zoospore production, four-day-old plates fully grown with $P$. palmivora were submerged with $10 \mathrm{ml}$ of $4{ }^{\circ} \mathrm{C}$ demi-water, and incubated at $25{ }^{\circ} \mathrm{C}$ for 30 minutes to release the zoospores. Successful release of zoospores was confirmed by microscopy. The zoospore suspension was filtered through a $50 \mu \mathrm{m}$ nylon mesh. The suspension was centrifuged for 5 minutes at $100 \mathrm{~g}$ to concentrate zoospores. After centrifugation, the zoospores were re-suspended in demi-water to a concentration of 1.105 zoospores $/ \mathrm{ml}$.

\section{Plant inoculation}

For inoculation of Medicago with mycelial plugs, 1 week old Medicago plants growing on Farhaeus plates were used. Mycelial plugs $(\varnothing \approx 3 \mathrm{~mm})$ were punched from V8 plates on which Phytophthora was cultured for 4 days. Plugs were placed on Medicago roots, with the mycelium side contacting the root. Inoculated plants were incubated at $25^{\circ} \mathrm{C}$ at a $16 / 6$ hour light/dark regime until analysis.

Before inoculation with zoospores, seedlings or root cultures were transferred to tubes containing $20 \mathrm{ml}$ demi-water. Root cultures were washed 3 times for 1 hour in demiwater to remove residual sugar-containing M-medium from their surface. The hypocotyls of seedlings were inserted into a plastic foam floater to prevent the cotyledons from submersion. For inoculation, $100 \mu \mathrm{l}$ of zoospore suspension was added. Inoculated plants were incubated at $25{ }^{\circ} \mathrm{C}$ under a $16 / 6$ hour light/dark regime until analysis. Eight tubes containing three seedlings each were used per mutant. Seedlings from each tube were examined at 2, 4 and 6 dpi.

\section{Mycorrizal inocculation}

An axenic Rhizophagus irregularis culture was maintained on a Medicago root culture growing on solid M medium (Bécard \& Fortin, 1988). The VAMP721d/e silenced root cultures and the empty vector control root cultures, growing on solid $\mathrm{M}$ medium, were inoculated by transferring hyphae and spores from the donor plate to the root cultures plates. After 4 weeks, the infection was analysed.

\section{Trypan Blue staining}

Roots were placed in Trypan Blue staining solution (60\% ethanol, 10\% phenol, $10 \%$ glycerol, $10 \%$ lactic acid, $10 \%$ water and $0.02 \%$ trypan blue) and heated to $60{ }^{\circ} \mathrm{C}$ for two hours. After staining, the roots were de-stained for 16 hours in chloral hydrate solution ( 2.5 $\mathrm{g} / \mathrm{ml}$ ), replacing the de-staining solution twice. After de-staining, the roots were mounted in glycerol on microscope slides for imaging. 


\section{WGA staining.}

Roots were cleared in $10 \% \mathrm{KOH}$ at $60{ }^{\circ} \mathrm{C}$ for two hours. Then, the roots were washed 3 times in PBS $\left(150 \mathrm{mM} \mathrm{NaCl}, 10 \mathrm{mM} \mathrm{Na}_{2} \mathrm{HPO}_{4^{\prime}} 1.8 \mathrm{mM} \mathrm{KH}_{2} \mathrm{PO}_{4^{\prime}} \mathrm{pH} 7.4\right)$, after which the roots were stained in $0.2 \mu \mathrm{g} / \mathrm{mL}$ WGA-Alexafluor 488 (Molecular Probes) in PBS at room temperature for 16 hours.

\section{Plastic embedding}

Before plastic embedding, roots were fixed by vacuum infiltrating $5 \%$ glutarealdehyde in $0.1 \mathrm{M} \mathrm{PO}_{4}$ buffer ( $\left.\mathrm{pH} 7\right)$. After fixation, the roots were dehydrated in an ethanol dehydration series (30\%, 50\%, 70\%, 1 hour each, followed by $100 \%$ ethanol for 16 hours) and embedded in Technovit 7100 (Heraeus-Kulzer). Sections of $6 \mu \mathrm{m}$ were cut using a microtome, and transferred to microscope slides. Sections were stained in $0.05 \mathrm{M}$ Toluidine Blue for 30 seconds and destained in water for 10 minutes.

\section{GUS staining}

Roots were harvested by cutting of the roots at the junction with the hypocotyl. Roots or root cultures inoculated with $P$. palmivora GUS were washed 3 times in $0.1 \mathrm{M} \mathrm{PO}_{4}$ buffer $(\mathrm{pH}$ 7). After washing, roots were submerged in GUS buffer ( $3 \%$ glucose, $5 \mathrm{mM}$ EDTA, $0.5 \mathrm{mM}$ ferrocyanide, $0.5 \mathrm{mM}$ ferricyanide, $0.5 \mathrm{~g} / \mathrm{I} \mathrm{X}$-gluc in $0.1 \mathrm{M} \mathrm{PO}_{4}$ buffer $(\mathrm{pH}$ 7)) and vacuum infiltrated for 30 minutes. The roots were incubated at $37^{\circ} \mathrm{C}$ for approx. 1 hour until a clear blue staining could be observed. The samples were cleared in $30 \%$ ethanol.

\section{Quantification of infection}

After GUS staining, the roots were imaged using a flatbed scanner. The length of blue staining in the entire root was measured using the measurement tool in ImageJ software. The significance of differences was determined using a Mann-Whitney $U$ test in the IBM SPSS 22 software.

\section{Microscopy}

Roots stained with Trypan Blue, GUS or embedded in plastic, were examined with a Leica DM5500 light microscope. Infection by P. palmivora GFP was analysed with a Zeiss LSM550 inverted confocal microscope. A $488 \mathrm{~nm}$ laser was used for excitation of GFP, propidium iodide and FM4-64. GFP emission was observed between $505 \mathrm{~nm}$ and $530 \mathrm{~nm}$, whereas propidium iodide and FM4-64 emission was observed between $560 \mathrm{~nm}$ and $650 \mathrm{~nm}$. 


\section{ACKNOWLEDGEMENTS}

We thank Sophien Kamoun for providing P. palmivora isolate 16830, and Giles Oldroyd and Jeremy Murray for the Medicago mutant lines ram2 and vapyrin, respectively. R.H., T.B. and E.L. are supported by the European Research Council (ERC-2011-AdG294790). K.B. is supported by a STW-VENI grant from The Netherlands Organization for Scientific Research.

\section{AUTHOR CONTRIBUTIONS}

R.H., K.B. and E.L. conceived and designed experiments. R.H., K.B. and M.B performed experiments. R.H., K.B. and E.L. analysed the data. R.H. K.B., F.G., T.B. and E.L wrote the manuscript. 


\section{SUPPLEMENTAL INFORMATION}

\section{Supplemental table S1. Primers used in this study}

Primer

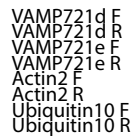

Sequence $5^{\prime}-3^{\prime}$

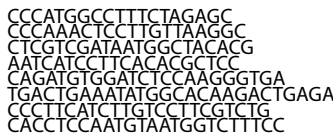

Supplemental table S2. Colonized root length of different Medicago genotypes.

\begin{tabular}{|c|c|}
\hline Genotype & $\begin{array}{l}\text { Colonized root } \\
\text { length }(\mathrm{cm})\end{array}$ \\
\hline A17 & $\begin{array}{l}4,13 \\
4,44 \\
3,82 \\
3,53\end{array}$ \\
\hline$n f p$ & $\begin{array}{l}2,79 \\
4,21 \\
3,62 \\
3,38\end{array}$ \\
\hline dmi2 & $\begin{array}{l}4,23 \\
4,59 \\
4,25 \\
4,00 \\
\end{array}$ \\
\hline dmi3 & $\begin{array}{l}3,71 \\
3,68 \\
2,24 \\
4,31 \\
\end{array}$ \\
\hline $\operatorname{ram} 2$ & $\begin{array}{l}4,05 \\
3,47 \\
3,77 \\
3,28 \\
\end{array}$ \\
\hline R108 & $\begin{array}{l}4,96 \\
4,32 \\
4,13 \\
5,34\end{array}$ \\
\hline$l y k 3$ & $\begin{array}{l}3,74 \\
4,32 \\
4,02 \\
3,79 \\
\end{array}$ \\
\hline$n s p 1$ & $\begin{array}{l}4,50 \\
3,91 \\
3,74 \\
3,86\end{array}$ \\
\hline$n s p 2$ & $\begin{array}{l}4,28 \\
4,44 \\
3,56 \\
2,81\end{array}$ \\
\hline$i p d 3$ & $\begin{array}{l}2,92 \\
4,22 \\
3,50 \\
3,62\end{array}$ \\
\hline$v p y$ & $\begin{array}{l}5,10 \\
5,34 \\
5,19 \\
4,06\end{array}$ \\
\hline
\end{tabular}




\section{Chapter 2}
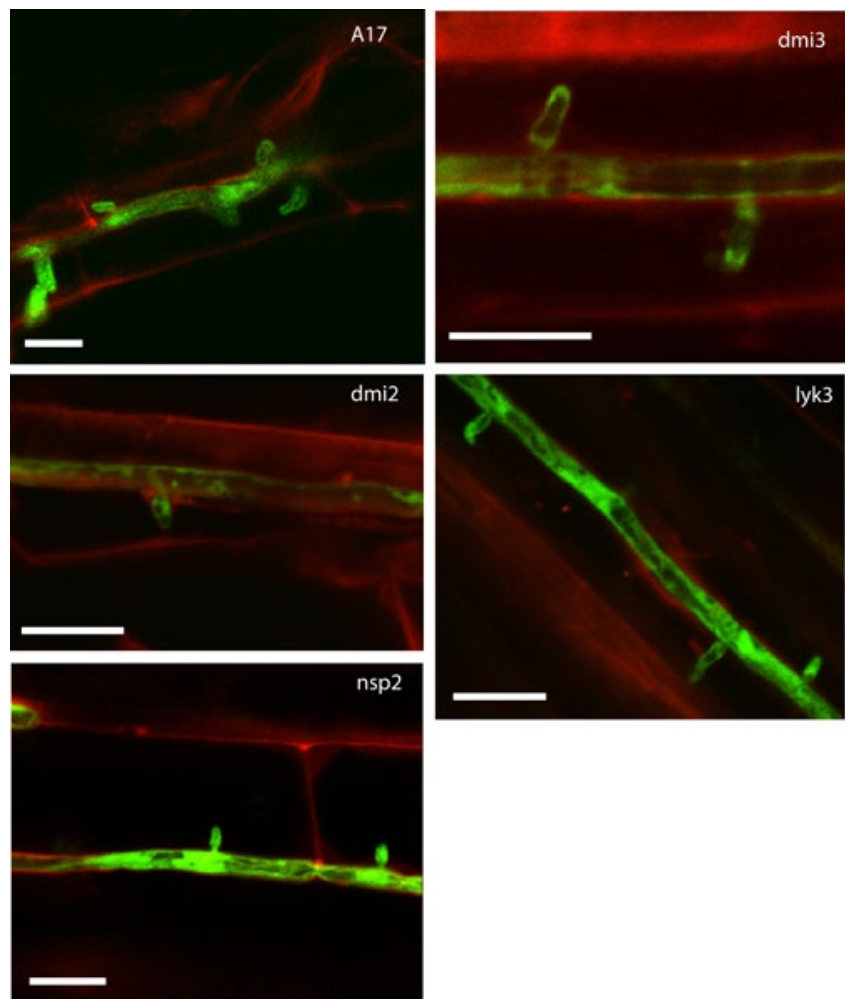

Figure S1. Haustorium formation in roots of Medicago wild type plants and symbiosis mutants

Confocal images showing infection of Medicago A17 (A) dmi2 (B) and dmi3 (C) with GFP labeled P. palmivora. Roots are counterstained with propidium iodide labeling cell walls. Haustoria (white arrowheads) formed in roots of Medicago ecotypes A17 and different Medicago mutants, 2dpi. scale bars represent $10 \mu \mathrm{m}$. 

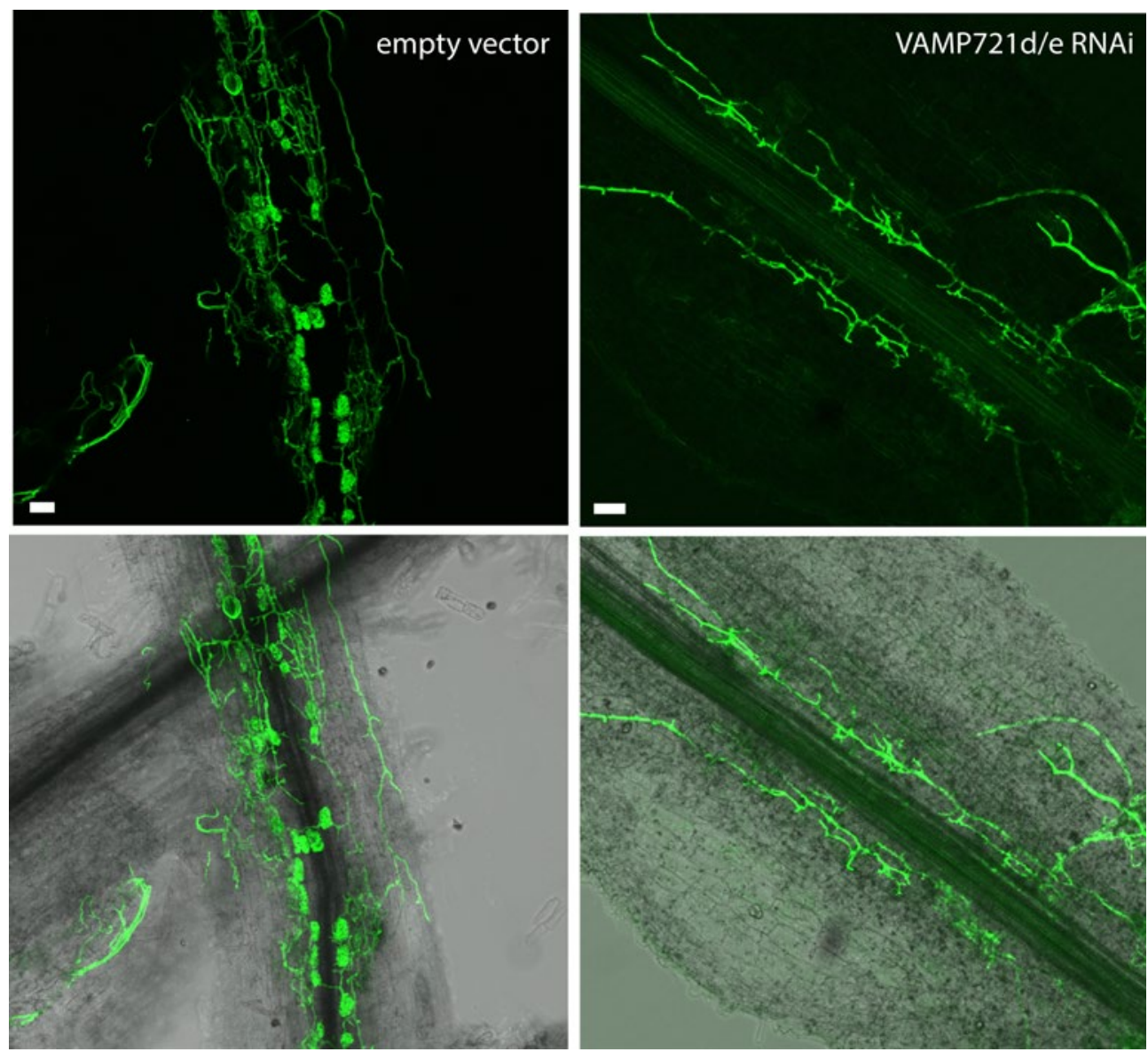

\section{Figure S2. Arbuscule formation is blocked in VAMP721d/e silenced root cultures.}

Projections of confocal image stacks showing WGA-alexa488 stained root cultures, 4 weeks after inocculation with AM fungus Rhizophagus irregularis. A, In the empty vector transformed control cultures, many arbuscules are formed. B, In the root cultures where VAMP721d and e are silenced, intraradicial colonization is observed, but no arbuscules are formed. scale bars represent $50 \mu \mathrm{m}$. 


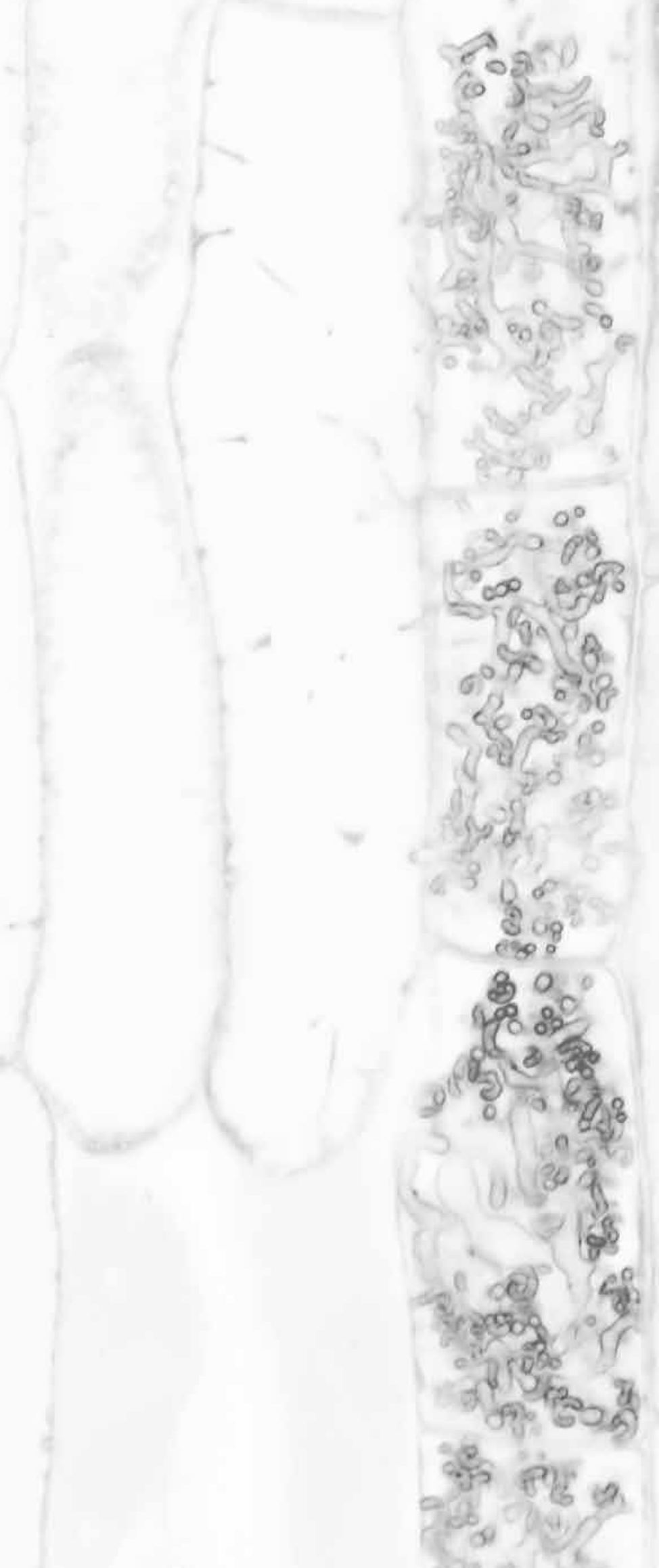




\title{
Chapter 3
}

\section{A symbiosis-dedicated SYNTAXIN OF PLANTS 13II isoform controls the formation of a stable host-microbe interface in symbiosis}

\begin{abstract}
- Arbuscular mycorrhizal (AM) fungi and rhizobium bacteria are accommodated in specialized membrane compartments that form a host-microbe interface. To better understand how these interfaces are made, we studied the regulation of exocytosis during interface formation.
\end{abstract}

-We used a phylogenetic approach to identify t-SNAREs that are dedicated to symbiosis and used cell-specific expression analysis together with protein localization to identify t-SNAREs that are present on the host-microbe interface in Medicago truncatula. We analyzed the role of these $t$-SNAREs during the formation of a host-microbe interface.

-We show that multiple syntaxins are present on the peri-arbuscular membrane. From these, we identified SYP13II as a t-SNARE that is essential for the formation of a stable symbiotic interface in both AM and rhizobium symbiosis. In most dicot plants the SYP13II transcript is alternatively spliced resulting in two isoforms, SYP13/Ia and SYP13 II. These spliceforms differentially mark functional and degrading arbuscule branches.

- Our results show that vesicle traffic to the symbiotic interface is specialized and required for its maintenance. Alternative splicing of SYP13II allows plants to replace a t-SNARE involved in traffic to the plasma membrane with a t-SNARE that is more stringent in its localization to functional arbuscules. 


\section{INTRODUCTION}

Plants interact with microbes to acquire scarce nutrients from the soil. Endosymbioses are the most intimate among these interactions, as the microbes are hosted within plant cells. Two of the best-studied examples are the symbiosis between plants and arbuscular mycorrhizal fungi and the rhizobium-legume symbiosis. Arbuscular mycorrhizal (AM) fungi are hosted inside root cortex cells in the vast majority of all land plants where highly branched hyphae form so-called arbuscules (Gutjahr \& Parniske, 2013) . Rhizobia induce the formation of root nodules on legume roots, where they are hosted intracellularly as nitrogen-fixing organelles called symbiosomes (Roth \& Stacey, 1989; Udvardi \& Poole, 2013). In both AM and rhizobial endosymbioses, the microbes are surrounded by a specialized host membrane, i.e. the peri-arbuscular membrane and symbiosome membrane respectively, which forms a symbiotic interface. These interfaces facilitate the efficient exchange of nutrients and signals between both partners, as they increase the contact area and are typically devoid of a structured cell wall (Balestrini \& Bonfante, 2014) . Furthermore, the interface membranes are equipped with specific nutrient transporters that are dedicated to symbiosis (Harrison et al., 2002; Kobae et al., 2010; Udvardi \& Poole, 2013). Although delivery of membrane and proteins to the host microbe interface and the plasma membrane both depended on exocytosis, both compartments have a distinguishable protein composition. Our aim is to understand how exocytosis is modified in plant cells to create a new specialized membrane compartment to host microbes.

It has recently been suggested that arbuscule formation involves a general, transient reorientation of exocytosis from the plasma membrane to the peri-arbuscular membrane (Genre et al., 2012; Pumplin et al., 2012; Ivanov \& Harrison, 2014). It was shown that timing of the expression of various transporters determines whether they localize to the plasma membrane or the peri-arbuscular membrane surrounding the hyphal fine branches. In addition, selection of cargo that enters the secretory pathway at the ER/trans-Golgi was shown to contribute to the specific targeting of proteins to the interface (Pumplin et al., 2012). On the other hand, it has been shown that formation of the host-microbe interface in both AM and rhizobium symbiosis requires a specific subset of exocytosis related vesicle SNAREs (Ivanov et al., 2012). SNAREs (N-ethylmaleimide-sensitive factor-attachment protein receptors) drive specific vesicle fusion by the formation of complexes between v-SNAREs on the vesicle and t-SNAREs on the target membrane (Lipka et al., 2007; Kwon et al., 2008). Two specific v-SNAREs, MtVAMP721-d and -e, were shown to be essential for interface formation in both endosymbioses, but not for root development or the formation of infection threads and arbuscular trunks; cell wall bound intracellular infection structures that precede the formation of the interfaces (Ivanov et al., 2012). This suggests that multiple exocytosis pathways marked by distinct SNAREs co-exist that may be differentially regulated to accommodate the (Ivanov et al., 2012). The recent identification of an exocyst subunit that exclusively occurs in plants that form an AM symbiosis and 
that is essential for the development of arbuscule fine branches supports the idea that vesicle traffic during microbial accommodation is specialized (Zhang et al., 2015b).

We hypothesized that the formation of a symbiotic interface depends on a symbiosis-dedicated membrane trafficking pathway that is regulated by SNAREs that are distinct from the SNAREs that mediate traffic to the plasma membrane. Therefore, we focused here on t-SNAREs involved in exocytosis. We show that multiple plasma membrane syntaxins are present on the symbiotic interfaces. Furthermore, we identify an evolutionarily conserved symbiosis-dedicated t-SNARE, SYNTAXIN OF PLANTS 13II that is alternatively spliced in most dicot plants and which is essential for the formation of a stable interface in both AM and rhizobium symbioses. In Medicago truncatula the two SYP13II spliceforms differentially label arbuscular fine branches and degrading parts of the interface, which supports the notion that vesicle traffic to the arbuscule interface during its formation and degradation is specialized and involves distinct syntaxins.

\section{RESULTS}

\section{Medicago SYP132 is alternatively spliced and encodes a symbiosis-dedicated syntaxin}

We used a phylogenetic approach to identify t-SNAREs that could mark an exocytosis pathway dedicated to symbiotic interface formation. This approach exploits the observation that genes that are dedicated to AM symbiosis are in general lost in plants species that have lost the ability to host AM fungi (Delaux et al., 2014). These genes include orthologs of the symbiotic v-SNAREs MtVAMP721- $d$ and -e (Ivanov et al., 2012). Therefore, we analysed the phylogeny of the four families of t-SNAREs associated with exocytosis: SYP1, SYP7, SNAP and NSPN (Sanderfoot, 2007). The protein sequences of these SNAREs were retrieved for the non-host Arabidopsis and the three AM host-species Medicago, Carica papaya and Solanum lycopersicum, and were used to construct phylogenetic trees (Fig. S2). In the SYP7, SNAP and NSPN families, all orthology groups contain at least one Arabidopsis member (Fig. S2). Within the SYP1 family that encompasses all exocytosis related Qa class t-SNAREs, one orthology group contains proteins from Medicago, Carica and Solanum, while no Arabidopsis ortholog is present (SYP13 clade II; Figure S2). This group contains Medicago SYNTAXIN OF PLANTS 132 (SYP132), which has been reported to be present on the symbiosome membrane in rhizobium symbiosis (Catalano et al., 2007; Limpens et al., 2009). The absence of a SYP132 ortholog in Arabidopsis as well as its occurrence on the symbiosome membrane suggests that it might be part of a symbiotic SNARE complex. 


\section{Chapter 3}

(a)

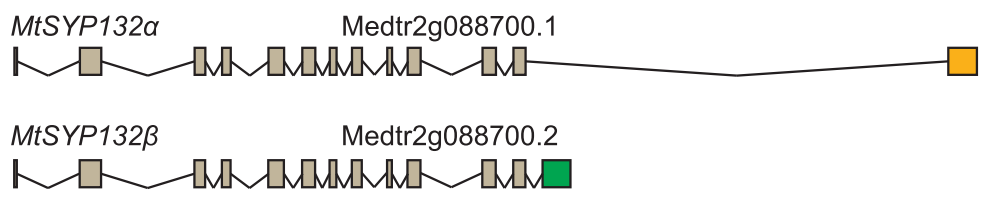

(b)

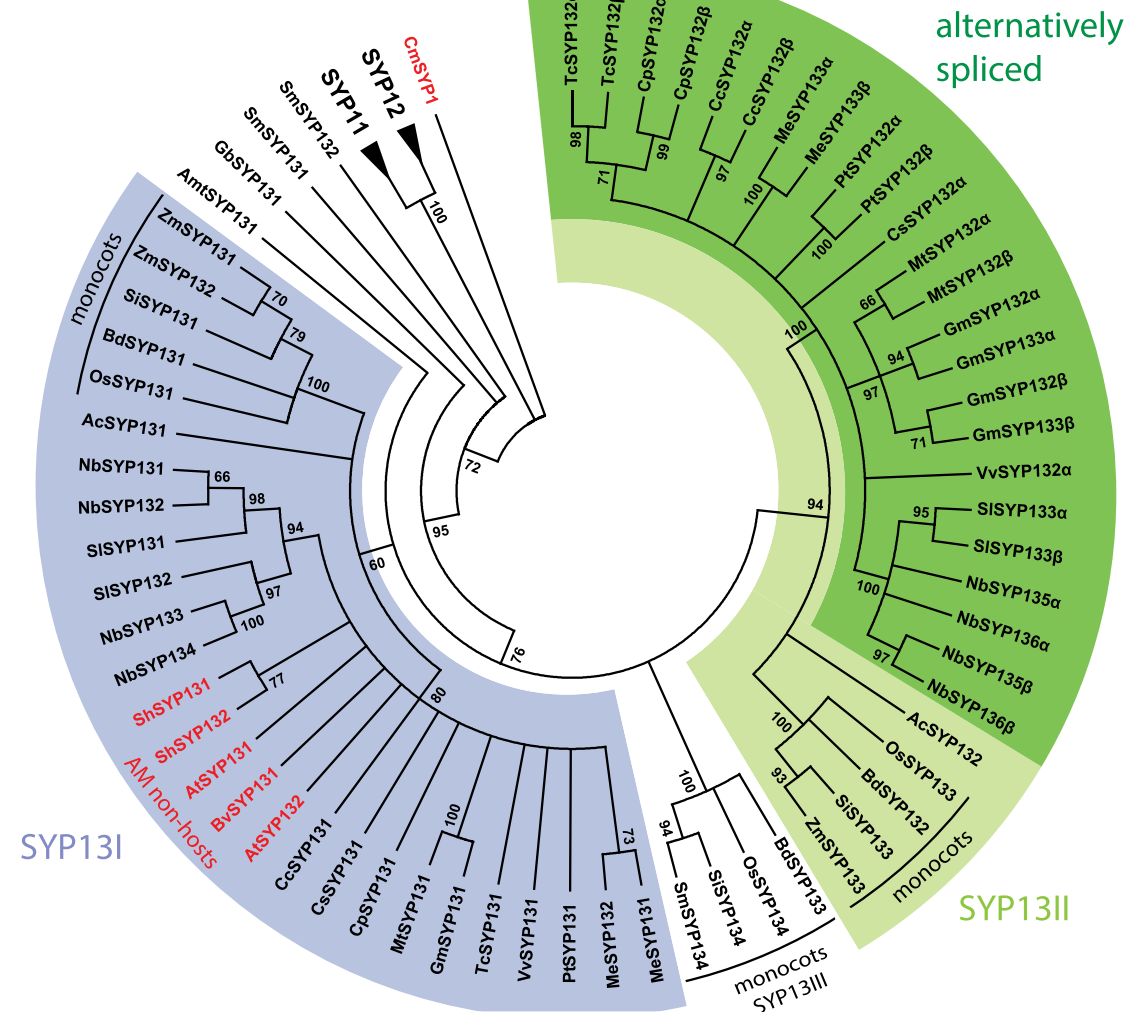

\section{Figure 1. Alternative splicing of SYP13/I is evolutionarily conserved in dicots}

(a) The two spliceforms of SYP132 are identical except for the use of a different C-terminal exon and polyadenylation site. Both encode full length syntaxins. (b) Maximum likelihood tree, showing the evolution of SYP13. The tree was rooted using Chlamidomonas reinhardtii (Cr) SYP1. Included species are Amborella trichocarpa (Amt), Aquilegia coelurea (Ac), Arabidopsis thaliana (At), Beta vulgaris (Bv), Brachypodium distachyon (Bd), Carica papaya (Cp), Citrus clementina (Cc), Cucumis sativus (Cs), Ginkgo biloba (Gb), Glycine max (Gm), Manihot esculenta (Me), Medicago truncatula (Mt), Nicotiana benthamiana (Nb), Oryza sativa $(\mathrm{Os})$, Populus trichocarpa (Pt), Setaria italica (Si), Selagionella moellendorfii (Sm), Solanum lycopersicum (SI), Striga hermonthica (Sh), Theobroma cacao (Tc), Vitis vinifera ( $\mathrm{Vv})$ and Zea mays $(\mathrm{Zm})$. Genes of species that are non-host to AM fungi are shown in red. For SYP11 and SYP12 only the species Arabidopsis thaliana, Carica papaya, Medicago truncatula and Solanum lycopersicum were included. 
We noted that two splice-forms of Medicago SYP132 are annotated, of which both annotations are supported by EST sequences. Alternative splicing of MtSYP132 results in the use of a different last exon, 3' UTR sequence and poly-adenylation site. Both spliceforms encode proteins that are predicted to be functional/full-length syntaxins. We named the two isoforms SYP132 $a$ and SYP132 $\beta$ (Fig. 1a). These syntaxins differ in their last 60 amino acids, encoding a small part of the SNARE domain and the adjacent C-terminal transmembrane domain. If the alternative splicing of SYP132 is functionally relevant for AM symbiosis, we expected it to be highly conserved among plant species that interact with AM fungi. To investigate this, we retrieved the sequences of SYP13 proteins from a wide range of plant species including hosts and non-hosts of AM fungi (Fig. 1b). We manually curated the annotation of all SYP132 orthologs to identify alternative spliceforms before constructing a phylogenetic tree. In line with the analysis described above, monocot and dicot SYP13 proteins diverged into two highly conserved clades that we named SYP13I and SYP13II. Additionally, monocots have another SYP13 clade that does not cluster with either SYP13I or SYP13II, so we named it SYP13III. All species possess at least one protein in clade I. In addition, AM hosts have at least one protein that clusters with Medicago SYP132 in clade II, whereas all analysed non-host species (Arabidopsis thaliana, Striga hermonthica and Beta vulgaris, which have independently lost the ability to establish an AM symbiosis) lack a clade II gene. In most analysed dicots SYP13II is alternatively spliced, with the exception of Cucumis sativus, Vitis vinifera, and the most basal sequenced eudicot Aquilegia coerulea (Fig. 1b and Fig. S3). Alternative splice-forms were also not found in monocot plants. This suggests that alternative splicing evolved early in the dicot lineage after the split of Aquilegia, and one spliceform has subsequently been lost in Cucumis and Vitis. The conservation of SYP13II and its alternative splicing exclusively in AM-hosts suggests that both are involved in AM symbiosis.

\section{Multiple syntaxins are expressed in arbuscular cells}

Based on the phylogeny we hypothesized that both SYP13II isoforms mark exocytosis pathways dedicated to symbiosis, which are active in parallel to other exocytosis pathways that are marked by other SYP1 paralogs. Therefore, we studied the expression of all plasma membrane syntaxins at a cellular resolution in Medicago roots to determine whether multiple syntaxins are active in cells that form an arbuscule. We isolated arbuscule-containing cortex cells as well as cortical cells from uninfected roots by laser capture microdissection (Fig. 2a-b). RNA was isolated from these cells and used to determine the relative expression of all SYP1 genes by qPCR. This showed that multiple SYP1 genes are active in individual cells (Fig. 2c). In arbuscular cells, four genes make up the majority of SYP1 transcripts; SYP121, SYP122 and both spliceforms of SYP132. The expression of SYP122 appears to go down during arbuscule formation, while SYP121 remains largely unchanged. Interestingly, the SYP132 $\alpha$ and SYP132 $\beta$ transcripts co-exist in both arbuscular and non-arbuscular cells, although their relative expression markedly differs. SYP132 $\beta$ is the dominant spliceform in uninfected cortical cells, whereas SYP132a is the dominant spliceform in arbuscular cells. 


\section{Chapter 3}
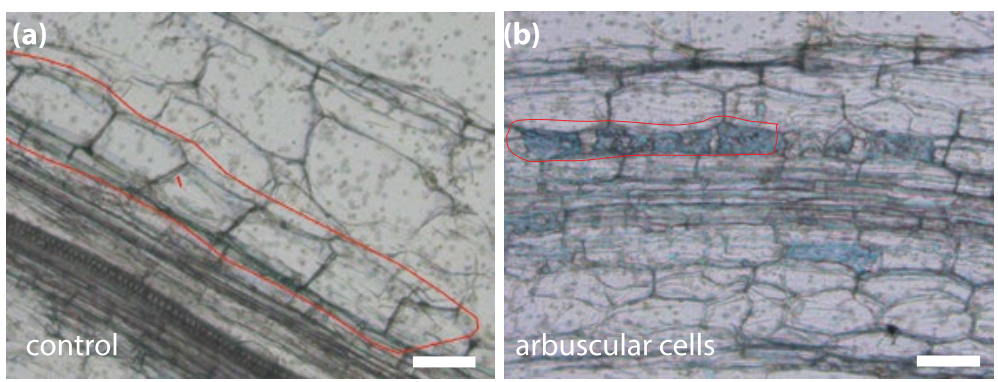

(c) $\square$ cortical cell

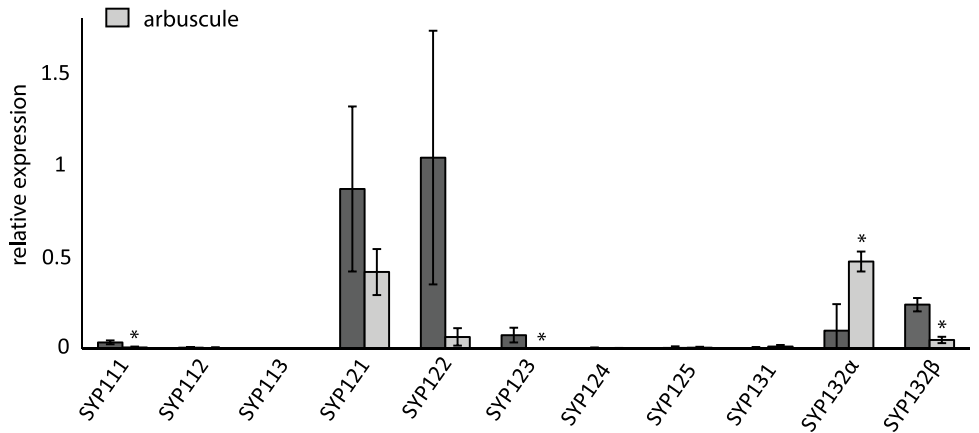

(d)

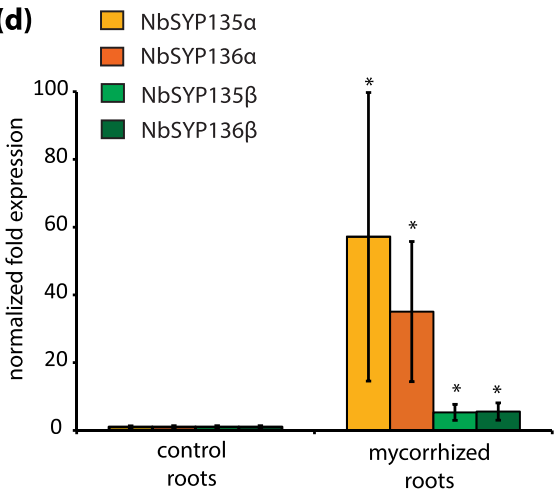

(e) $\square$ MtSYP132a
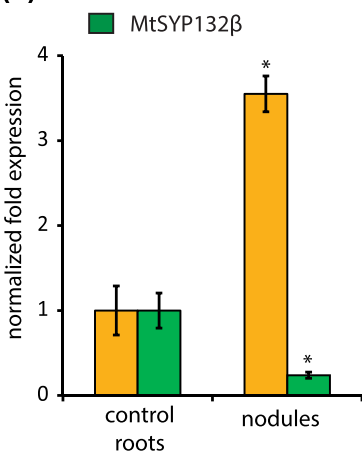

\section{Figure 2. Expression of SYP13/la correlates with symbiotic interface formation}

(a-b) $20 \mu \mathrm{m}$ thick sections of roots of Medicago truncatula, stained with Chlorazol black E, and embedded in steedmans wax. Using laser capture microdissection, we captured cortical cells from uninfected roots (a), or arbuscular cells from mycorrhized roots (b). scale bars are $50 \mu \mathrm{m}$ (c) qRT-PCR measurement of expression of SYP1 family members in uninfected cortical cells and arbuscular cells. The expression of different SYP1 orthologs is shown relative to the expression of three different reference genes (Actin2, Ubiquitin 10 and UBC9). Data are represented as the mean of 3 biological replicates \pm standard deviation. Asterisks indicate a significant difference between the two cell types (Students t-test, $p<0.05$ ). (d) qRT-PCR measurement of expression of SYP13II spliceforms of Nicotiana benthamiana in mycorrhized versus non-symbiotic control roots. Data are represented as the mean of 3 biological replicates \pm standard deviation. Normalized using NbActin as reference gene. Asterisks indicate a significant difference (Students t-test, p<0.05). (e) qRT-PCR measurement of expression of SYP132 spliceforms in 2 weeks old Medicago nodules compared to non-infected roots. Data are represented as the mean of 3 biological replicates \pm standard deviation. Asterisks indicate a significant difference (Students t-test, $\mathrm{p}<0.01$ ). 
To further link alternative splicing of SYP13/l to symbiosis, we studied the expression of both SYP13/l splice variants during AM symbiosis in Nicotiana benthamiana and during rhizobium symbiosis in Medicago by qRT-PCR. Like in Medicago, the transcript levels of Nicotiana SYP13lla were significantly increased after mycorrhizal infection relative to non-infected control roots (Fig. 2d), indicating that alternative splicing during mycorrhization is conserved in evolution. Also in Medicago nodules, SYP132a expression was significantly increased compared to roots (Fig. 2e). The change in expression of SYP132 $\beta$ was variable between different symbiotic interactions or plant species, although the splicing consistently shifted towards SYP132a in symbiotic tissues. Taken together, these data show that multiple syntaxins are present in cells that form a symbiotic interface and that the alternative splicing of SYP13/I is conserved among distant dicot species and the switch to a-splicing correlates with endosymbiosis.

\section{Medicago SYP121 and SYP122 are not required to form a host-microbe interface}

To test the role of exocytosis mediated by different SNAREs in symbiosis, we analysed the symbiotic phenotype in mutants of Medicago SYP121, SYP122 and SYP132, which represent the most dominantly active syntaxins in arbuscule containing cells and root nodules. Therefore, we obtained Tnt1 retrotransposon insertion lines for all three genes. We were unable to obtain homozygous plants of the SYP132 insertion line, which contains an insertion in the first intron (R108 line NF5183), suggesting that SYP132 is required for plant reproduction or development. For SYP121 and SYP122 we obtained lines that contain an insertion in their first exon (NF18082 and NF13214 respectively). To test their ability to form a host-microbe interface both homozygous insertion lines were inoculated with Rhizophagus irregularis and rhizobium strain Sinorhizobium meliloti Sm2011. After inoculation with AM fungi, highly branched arbuscules were formed in both mutant lines, similar to wild-type (Fig. S4a-c). Furthermore, nodules were formed on both mutant lines. The number of nodules as well as their ontology, containing fully differentiated symbiosomes, was similar to wild-type (Fig S4d-g). So exocytosis mediated by SYP121 or SYP122 is not essential to form a host-microbe interface although a potential redundant role of both SYP12 members cannot be excluded.

\section{Medicago SYP132 $a$ is essential for the formation of a stable host-microbe interface}

As a knock-out of SYP132 appeared to be lethal, we used an RNAi approach to examine the roles of the two MtSYP132 isoforms in symbiosis. We knocked down the individual splice-forms using RNAi constructs that only target the alternative last exons. Composite Medicago plants were generated via Agrobacterium rhizogenes-mediated root transformation and transgenic roots were selected based on the co-expression of a red fluorescent marker protein (Limpens et al., 2004). 


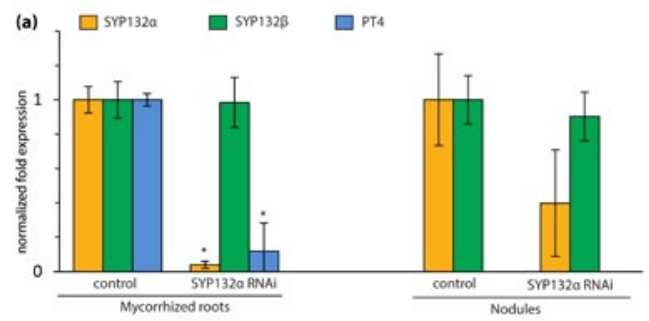

(e)
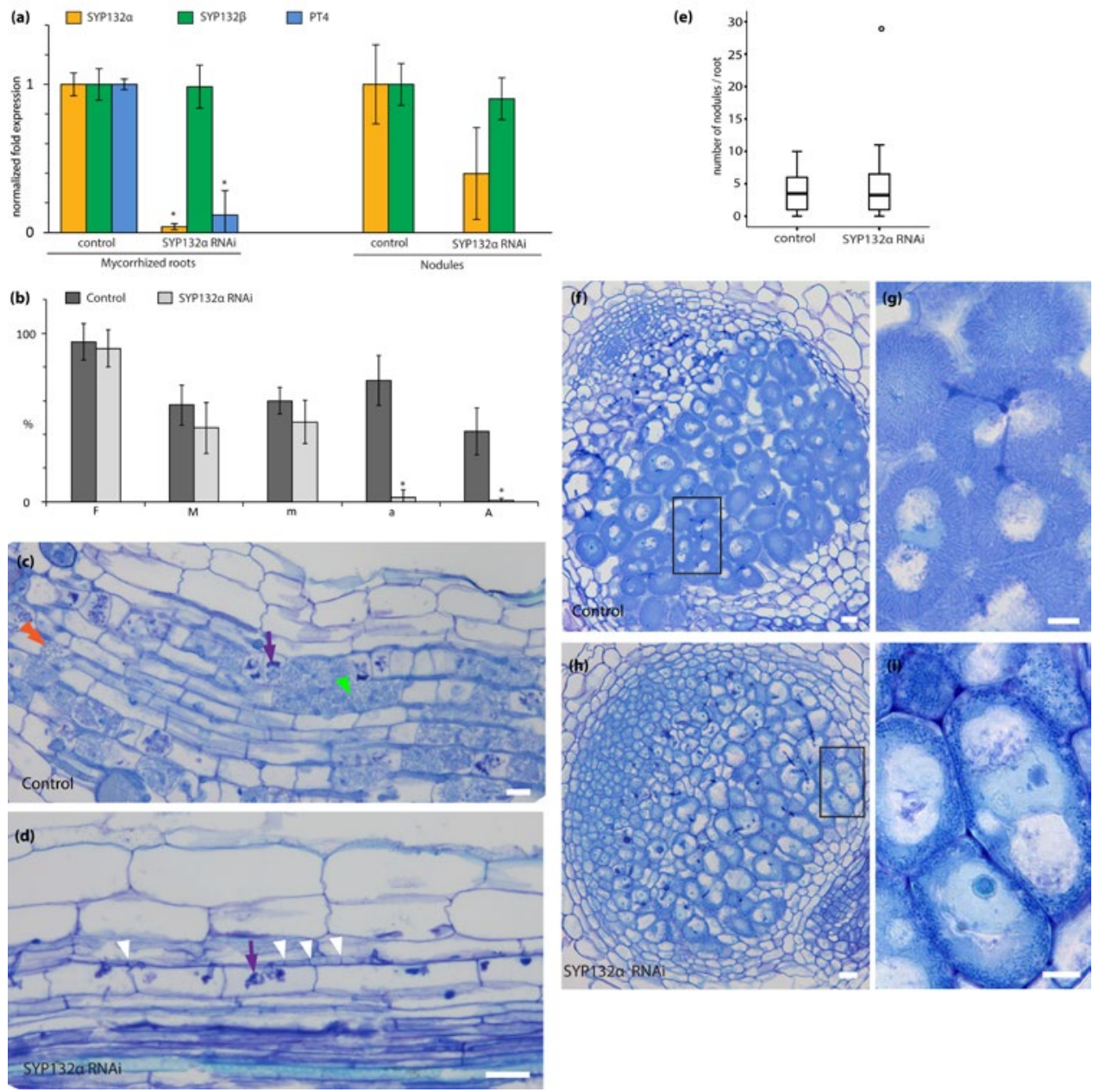

Figure 3. Medicago truncatula SYP132 $\alpha$ is essential for the formation and maintenance of an endosymbiotic interface.

(a) qRT-PCR showing specific knockdown of MtSYP132a in SYP132a RNAi roots relative to roots transformed with an empty vector, in mycorrhized roots $(n=6)$ and nodules $(n=7)$. Data are represented as the mean of biological replicates \pm standard deviation. Asterisks indicate a significant difference (Students t-test, $\mathrm{p}<0,01$ ). (b) quantification of mycorrhization of SYP132a RNAi roots compared to control roots. Variables according to Trouvelot et al. (Trouvelot et al., 1986) in \% are F, the frequency of analyzed root fragments that are mycorrhized; $M$, the intensity of infection; $m$, the intensity of infection in mycorrhized root fragments; $a$, mature arbuscule abundance in mycorrhized root parts; $A$, mature arbuscule abundance in the total root system. Data are represented as the mean of 8 biological replicates \pm standard deviation. Asterisks indicate a significant difference (Mann-Whitney $\mathrm{U}$ test, $\mathrm{p}<0.01)$ (c) Semi-thin $(6 \mu \mathrm{m})$ section of 4-week old mycorrhized control root. Different stages of arbuscules are visible, from young developing arbuscules (double orange arrowhead), mature arbuscules (green arrowhead), to degenerating arbuscules (purple arrow) scale bar $=25 \mu \mathrm{m}$. (d) Semi-thin section of mycorrhized SYP132a RNAi root. Only degenerating arbuscules are present (purple arrow). Septae are visible within hyphae (white arrowhead). Scale bar $=25 \mu \mathrm{m}$. (e) Number of nodules formed on control roots and SYP132a RNAi root, 14 days post inoculation. No significant difference was found (Mann-Whitney U test). (f) Semi-thin section of a nodule (14 dpi) on control root. Scale bar $=25 \mu m$. (g) Magnification of $\mathrm{f}$, differentiated symbiosomes are radially oriented around the vacuole. scale bar $=10 \mu \mathrm{m}$. (h) Semi-thin plastic section of a nodule (14 dpi) showing impaired differentiation/elongation of symbiosomes. Scale bar $=25 \mu \mathrm{m}$. (i) Magnification of $\mathrm{h}$. bacteria are released from infection threads, but do not differentiate. Scale bar $=10 \mu \mathrm{m}$. 
On plants transformed with a construct targeting MtSYP132 $\beta$, only few fluorescent transgenic roots were obtained 21 days post transformation (Fig. S5a). In the roots that did form, knock-down levels were less than 50\% (Fig. S5b), indicating selection for roots with low RNAi efficiency. In contrast, the amount of transgenic roots containing a RNAi construct that targets SYP132a was similar to that on plants transformed with an empty vector (Fig. S5a). In these roots, the silencing of SYP132a was efficient and specific (Fig. S5b). The transgenic roots displayed a normal growth rate, with a root length equal to control plants, 21 days post transformation (Fig S5c). This suggests that SYP132 $\beta$ is essential for Medicago root development, which is in line with our inability to obtain homozygous plants for retrotransposon insertion in SYP132, whereas SYP132a is not.

Four weeks after inoculation with $R$. irregularis, an efficient and specific knockdown of SYP132a was observed with SYP132a expression levels down to $4 \%$ of the transcript abundance in control roots, while SYP132 $\beta$ expression was not affected (Fig. 3a). Both control roots as well as SYP132a RNAi roots were well colonized with an equal intensity of mycorrhization (Fig. 3b). In control roots, many fully developed arbuscules were observed (Fig. 3c), whereas mature arbuscules were nearly absent in RNAi roots $(0,8 \%$ compared to $42 \%$ in control roots; Fig. 3 b). Semi-thin sections of mycorrhized roots showed that arbuscule formation in RNAi roots did not progress to maturation. The arbuscule trunks formed in SYP132a RNAi roots like in roots transformed with an empty vector, but unlike in control roots the fine branches did not expand to completely fill the cell. we found many small clumps, indicating that the arbuscules did not develop to their full size, or collapsed prematurely (Fig. 3d). Furthermore, the expression of phosphate transporter MtPT4, a marker gene for arbuscule containing cells (Javot et al., 2007), was significantly reduced in SYP132a RNAi roots ( $p<0.01$; Fig. 3a).

Additionally, we examined the effect of SYP132a knock-down on symbiosome formation in root nodules. Two weeks after inoculation with an S. meliloti strain expressing GFP driven by the NifH promoter, a similar amount of nodules was formed on control and SYP132a RNAi roots (Fig. 3e). The RNAi efficiency in nodules was highly variable with SYP132a transcript levels ranging from $10 \%$ to $100 \%$ of the expression in control nodules (Fig. $3 a$ ). SYP132 $\beta$ expression levels were not affected in these nodules. Because of the variability in silencing efficiency, we used absence of GFP expression as a pre-selection for disturbed nodule phenotypes. The activity of the $\mathrm{NifH}$ promoter reflects the presence of nitrogenase in mature nitrogen fixing rhizobia, allowing a quick distinction between nitrogen fixing GFP positive nodules and non-fixing GFP-negative nodules. On SYP132a RNAi roots around $20 \%$ (55/288) of the nodules were GFP-negative versus 5\% (13/249) of the nodules on control roots, (a significant difference; Chi square, $\mathrm{p}<0.01$ ). In nodules on control roots, bacteria were released and differentiated into mature symbiosomes that fill the cells (Fig. $3 f-g)$. In most GFP-negative nodules on SYP132a RNAi roots rhizobia did not differentiate/ elongate after release (Fig. 3h-i; 27/34 analyzed), whereas in some nodules also release of 
bacteria was blocked (3/34). GFP-negative nodules on control roots represented young primordia in which fixation did not yet start.

These data show that SYP132a is essential for interface formation and maintenance in both AM and rhizobia symbioses. None of the other plasma membrane syntaxins that are expressed in arbuscular cells are sufficient to rescue interface development when SYP132a is silenced. Especially SYP121 is expressed at similar levels as SYP132a in arbuscular cells (Fig. 2c). This suggests that different SYP1 members mark different exocytosis pathways, of which the pathway marked by SYP132a is specifically required to form a stable host-microbe interface.

\section{Multiple syntaxins label the symbiotic host-microbe interface}

The inability of the other SYP1 members to rescue interface formation in the absence of SYP132a could be due to their inability to mark the arbuscule fine branches or symbiosome membranes. To test this, we studied the sub-cellular localization of SYP121, SYP122 and the two isoforms of SYP132 during symbiosis. We expressed GFP fusion constructs of all four SNAREs driven by their native promoter in Medicago plants and checked their localization using confocal microscopy. All four proteins localized to both the plasma membrane, the arbuscule trunk as well as the peri-arbuscular membrane surrounding the fine-branches, during AM symbiosis (Fig. 4a-d). In contrast to the other syntaxins, SYP122 labelled the arbuscular trunk domain stronger compared to the fine branches, which correlates with its transcriptional downregulation upon arbuscule formation (Fig. 2c).

In nodules, fluorescence from the SNARE constructs driven by their respective native promoters was too weak to reliably assess their sub-cellular localization in the infected cells. This might be caused by the lower oxygen tension in these cells, which lowers GFP fluorescence (Limpens et al., 2009). In the nodule meristem all four constructs localized to the plasma membrane (data not shown). To determine the subcellular localization of the proteins in infected nodule cells, we therefore used the constitutively active Arabidopsis EF1a promoter and/or the Pisum sativum Leghemoglobin promoter that is active in infected cells. All four syntaxins localized to both the plasma membrane and the symbiosome membrane throughout its development (Fig. 4e-j). These data show that the different syntaxins do not spatially mark separate domains that distinguish the symbiotic interface from the plasma membrane, arbuscule trunk domain or infection threads. 

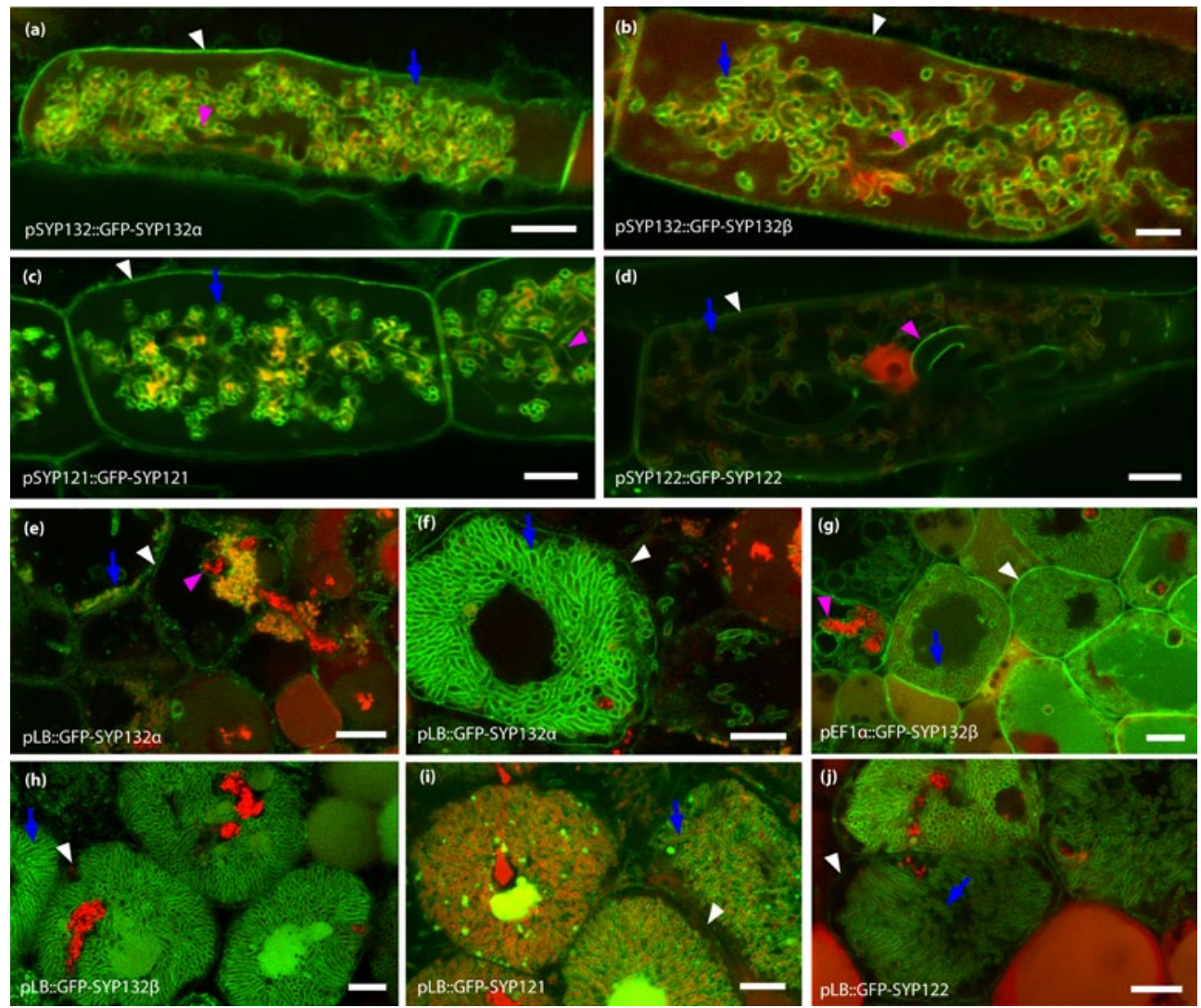

\section{Figure 4. Multiple t-SNAREs are present on the host-microbe interface.}

(a-d) Confocal laser scanning images, showing the localisation of GFP labeled syntaxins expressed from their native promoter in arbuscular cells in Medicago truncatula roots. The cytoplasm is marked by dsRed expressed from the Arabidopsis Ubiquitin3 promoter. All four syntaxins are present on the plasma membrane (white arrowhead), peri-arbuscular membrane (blue arrow), and the membrane surrounding the trunk (violet arrowhead). scale bars are $10 \mu \mathrm{m}$. (e-j) Localization of GFP labelled syntaxins, expressed from the Pisum sativum leghemoglobin or Arabidopsis EF1a promoter, in infected cells of nodules. All four syntaxins are present on both the symbiosome membrane (blue arrow) as well as the plasma membrane (white arrowhead). Both isoforms of SYP132 are present on the infection thread during release of rhizobia (violet arrowheads; e, g). RFP labeled bacteria and dsRed marked cytoplasm are visible in red, although the signal decreased after release of bacteria due to lower oxygen tension. scale bars are $10 \mu \mathrm{m}$. 


\section{SYP13Ila is the ancestral symbiotic SYP13II isoform}

Since both SYP132 isoforms are evolutionarily conserved in most dicot AM hosts, we questioned what the difference is between the two proteins. Therefore, we analysed the amino acid sequence of the differentially spliced last exon in more detail. To pinpoint the amino acid residues that make up the essential differences between the two spliceforms, we compared the amino acid sequences of different plant species, to identify conserved sequence signatures characteristic for either SYP13lla or SYP13॥ß (Fig. 5a, Fig. S3). We observed that most differences between the two spliceforms are located around the transmembrane domain. This suggests that both spliceforms interact with different regulatory proteins at the membrane or that they are affected in their targeting or turn-over at the interface.

The spliceform specific-sequence signatures allowed us to track the evolution of SYP13II. The a-signature is similar to the sequence of the SYP13 clade II orthologs from monocots and Aquilegia, indicating that SYP13Ila is the ancestral isoform, and SYP13II $\beta$ has evolved later in the dicots. SYP13lla has been retained in both Cucumis and Vitis that have lost one spliceform (Fig. S3), consistent with its indispensable role in symbiosis. Furthermore, the loss of SYP132 $\beta$ in Cucumis and Vitis, together with the observation that non-AM hosts have lost both spliceforms indicates that the essential role of Medicago SYP132 $\beta$ in root/ plant development is apparently not strictly conserved in the dicot lineage. We observed that the sequence signature of SYP132 $\beta$ is similar to that of SYP13I, the sister clade of SYP13II. Therefore, we hypothesize that SYP132 $\beta$ evolved after an unequal crossover between the ancestral SYP13I and SYP13II early in the dicot lineage. So the evolution of SYP13II $\beta$ created a chimera of the symbiosis-dedicated SYP13II and SYP13I (Fig 5b). 
(a) SNARE domain Transmembrane domain

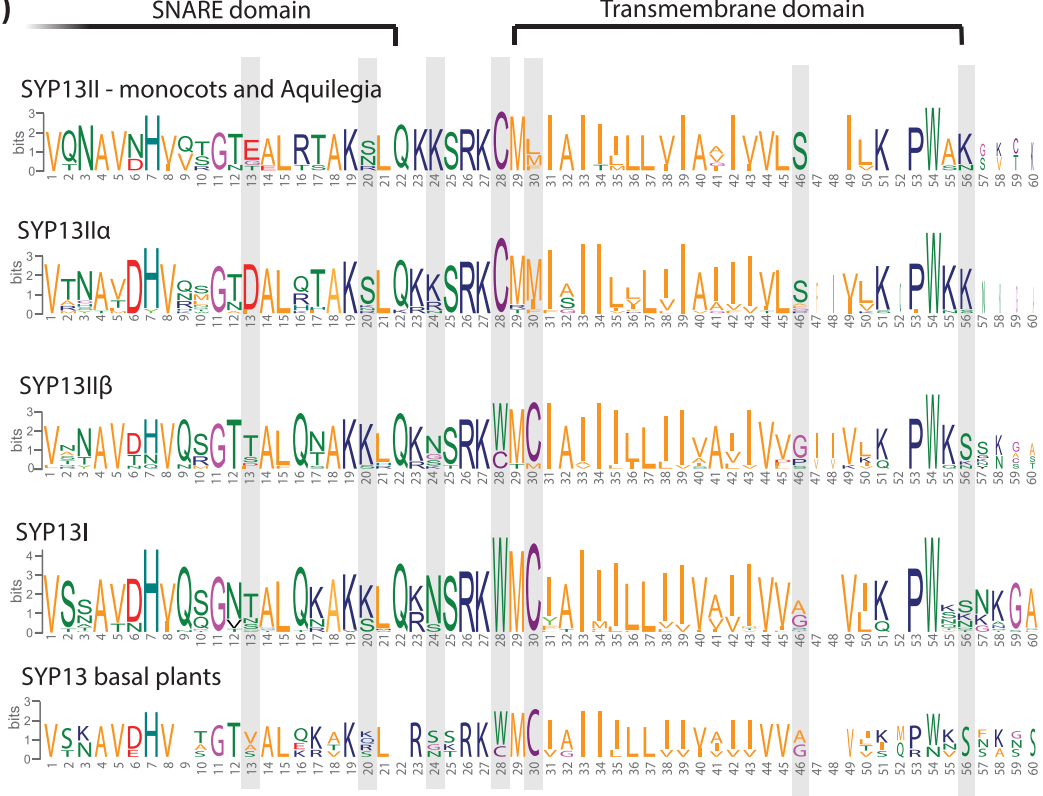

(b)

)

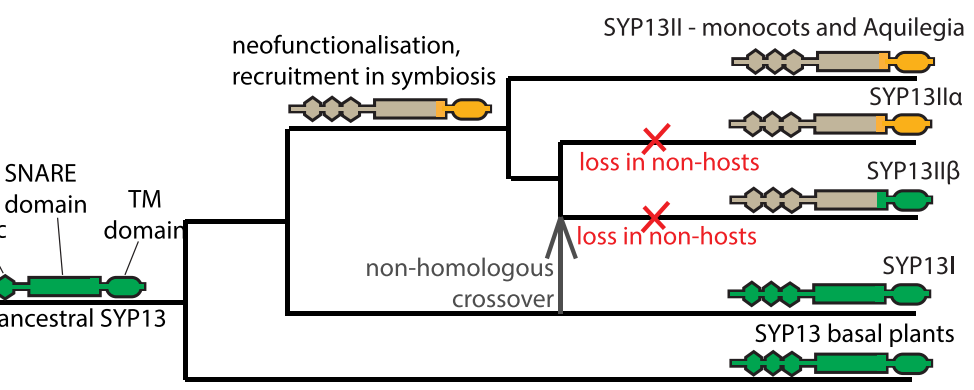

Figure 5. The evolution of SYP13II.

(a) Consensus sequences of the last exon of SYP13 genes from different plant species listed in figure 1b (see also Fig. S3). The height of characters reflects their bit score/level of conservation between species. Grey boxes indicate amino acid residues that are conserved among species, but different between a and $\beta$-splicing. (b) Proposed evolutionary scenario for SYP13 genes, based on the SYP13 phylogeny presented in Fig. $1 \mathrm{~b}$ and the comparison of the last exon sequences in panel a. Early in the angiosperm lineage, a gene duplication resulted in the non-symbiotic SYP13/ and SYP13/l that acquired a role in symbiosis. In dicots, after the split of Aquilegia, alternative splicing evolved, likely from an unequal crossover event between SYP13/ and SYP13II, creating SYP13II. Subsequently, the SYP13/l gene was lost in species that lost the ability to host AM fungi. 


\section{SYP132 $\beta$ retained the ancestral SYP13II function in arbuscule formation}

To understand the biological relevance of alternative splicing of SYP132, we questioned whether the proteins encoded by the two spliceforms are functionally different. Therefore, we investigated whether the SYP132 $\beta$ isoform can make up for the loss of SYP132 $\alpha$, if it is expressed at similar levels as SYP132a. To achieve this, we made a construct carrying the SYP132a RNAi cassette complemented with SYP132 $\beta$ expressed from its endogenous promoter. As a positive control, we complemented SYP132a RNAi using SYP132a itself. The last exon of both expression cassettes were codon-scrambled, to avoid silencing by the RNAi construct and to allow the independent detection of endogenous and transgene transcripts. After inoculation with R. irregularis, arbuscule morphology was fully restored in the SYP132a RNAi plants complemented with SYP132a and indistinguishable from plants transformed with an empty vector (Fig. 6). Strikingly, also in SYP132a RNAi plants complemented with SYP132 $\beta$ the arbuscule phenotype was restored. Thus, SYP132 $\beta$ protein has retained the ancestral ability of SYP13II to function in arbuscule formation.

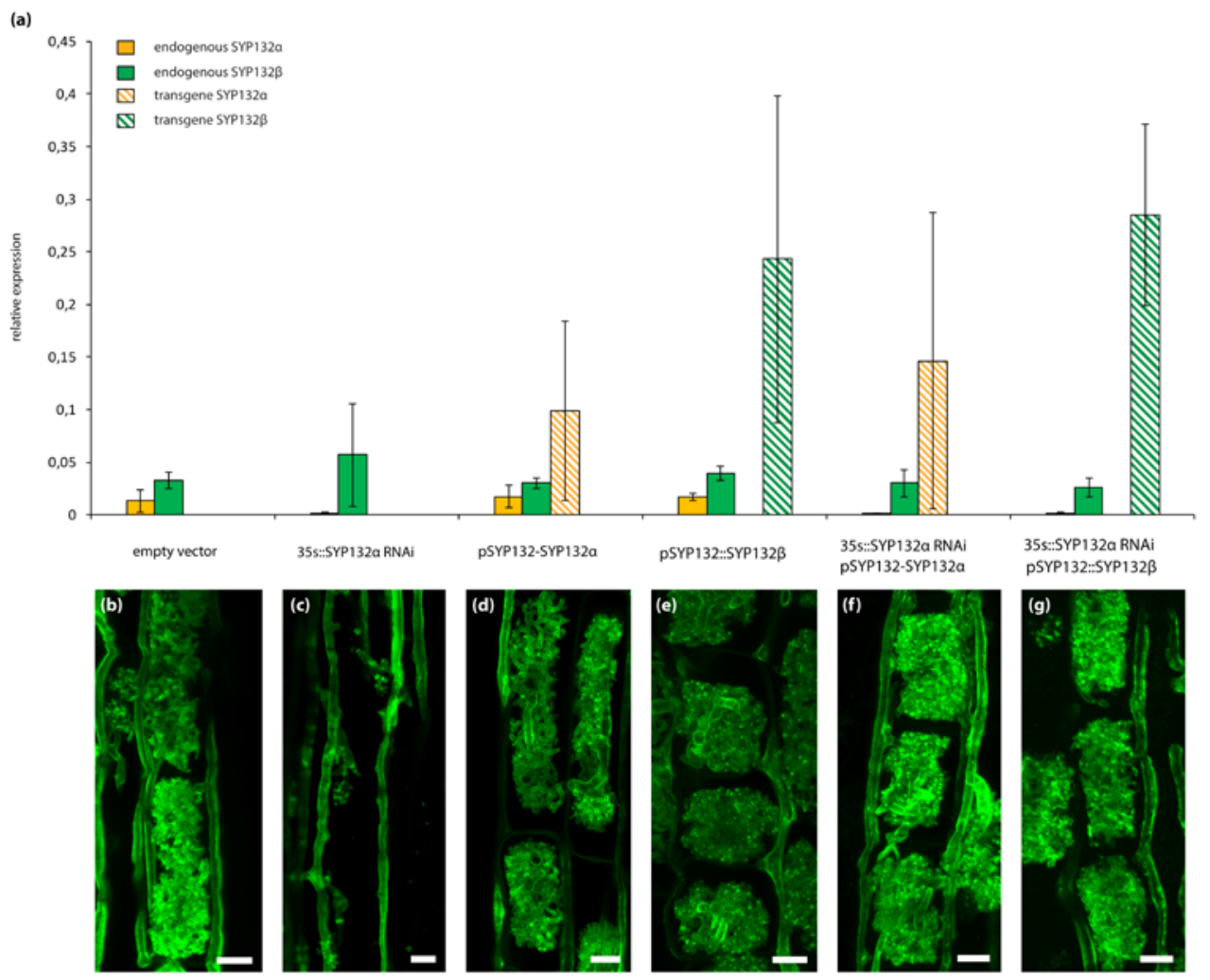

Figure 6. Medicago truncatula SYP132 $\beta$ is able to function in interface formation.

(a) qRT-PCR measurement of the endogenous and transgenic expression of both SYP132 isoforms, relative to reference gene MtActin2, in mycorrhized roots transformed with different constructs. Data are represented as the mean of 5 biological replicates \pm standard deviation. (b-g) WGA-alexafluor 488 stained mycorrhized roots transformed with constructs carrying different combinations of SYP132a RNAi cassettes and codon scrambled expression cassettes. scale bars are $10 \mu \mathrm{m}$. 


\section{SYP132 $\alpha$ and $\beta$ differentially localize to degenerating arbuscules}

Since the alternative splicing of SYP13II has been conserved since the split of Asterids and Rosids around 100 million years ago (Moore et al., 2010), and is tightly correlated with symbiosis, we argue that it is likely to be biologically relevant for mycorrhizal symbiosis. Since the main differences between the two isoforms were found around the transmembrane domain, we decided to re-analyse the subcellular localization of the two spliceforms in more detail and included later stages of arbuscule development.

Therefore, we co-expressed both spliceforms using the PT4 promoter, one tagged with GFP and the other tagged with mCherry in a single binary construct. This allowed the simultaneous localization of both isoforms in arbuscule cells. In cells that contained young or mature arbuscules, both isoforms labelled the arbuscule fine branches and we did not observe a difference in the localization between SYP132 $\beta$ and SYP132a (Fig. S6) We did, however, observe a striking difference in localization between SYP132 $\alpha$ and SYP132 $\beta$ at degenerating parts of the arbuscules, which was clearly visible in cells that contained partially degrading arbuscules. In these cells, SYP132 $\beta$ clearly labels the degrading part of the arbuscule, while its presence on the functional part largely decreases (Fig. 7a-c). The accumulation pattern of SYP132a is complementary as it preferentially accumulates on the non-degrading arbuscular fine branches. Swapping mCherry and GFP, confirmed the differential localizations (Fig. S6). To quantify the difference, we measured the fluorescence intensity of 32 different cells harbouring partially collapsing arbuscules, and we compared the abundance of each spliceform on functional and collapsing arbuscules. This showed that SYP132 $a$ is significantly more abundant on functional arbuscules, while SYP132 $\beta$ is significantly more abundant on collapsing arbuscules (Fig7 d-e). So, there is a clear difference in localization between the two spliceforms when both are expressed from the same promoter. This reveals that their subcellular localization is differentially regulated at a post-translational level when arbuscule degradation is initiated and cannot be attributed to timing of expression. 


\section{Chapter 3}

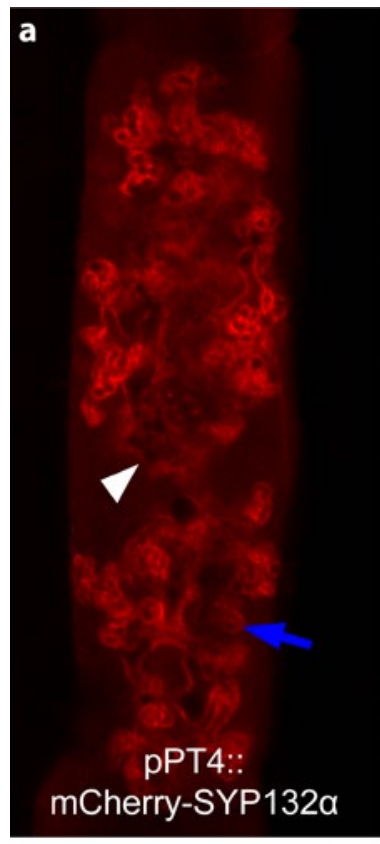

(b)

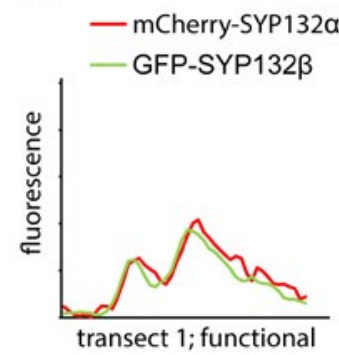

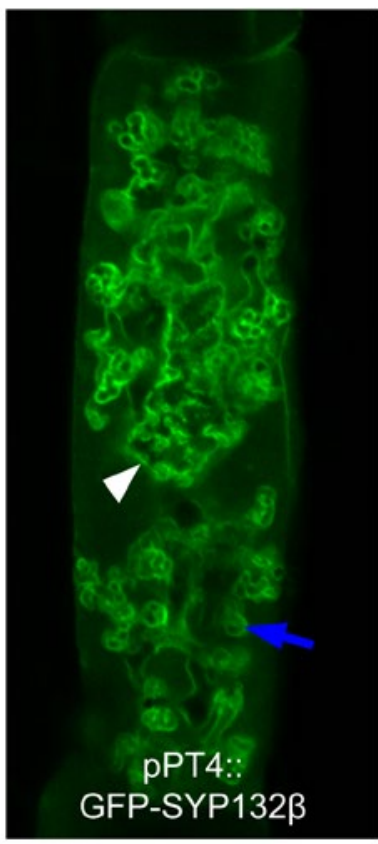

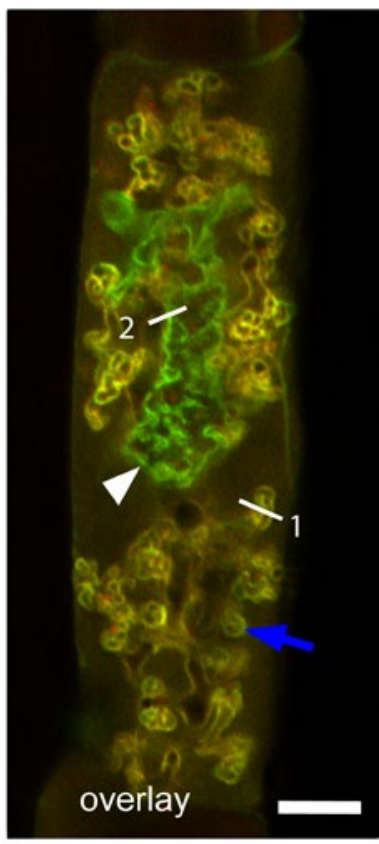

(c)
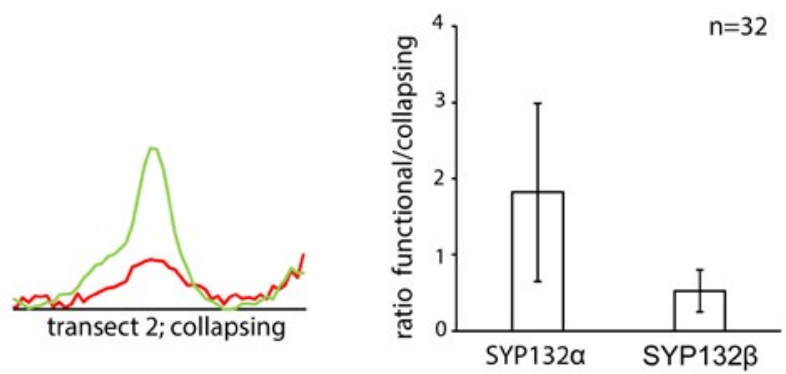

Figure 7. MtSYP132 $\alpha$ and $-\beta$ show different subcellular localization during arbuscule degeneration.

(a) Co-expression of GFP-MtSYP132 $\beta$ and mCherry-MtSYP132 $\alpha$ expressed from the MtPT4 promoter in Medicago truncatula roots. A difference in fluorescence intensity was observed between functional arbuscule branches (blue arrow) and collapsing arbuscule branches (whit arrowhead). Scale bar is $10 \mu \mathrm{m}$. (b) graphs showing fluorescence intensity in transects of functional and collapsing arbuscules, marked in panel a. (c) quantification of the ratio of fluorescence on functional arbuscules compared to collapsing arbuscules for both SYP132 $\alpha$ and SYP132 $\beta$. Data are represented as the mean \pm standard deviation. 32 partially collapsing arbuscules were analysed, using different combinations of fluorophores and spliceforms. SYP132a is significantly more abundant on functional arbuscules compared to collapsing arbuscules (Wilcoxon signed rank test, $p<0,01$ ), whereas SYP132 $\beta$ is more abundant on collapsing arbuscules compared to functional arbuscules (Wilcoxon signed rank test, $p<0,01$ ). Note that the ratio of SYP132 $\alpha$ and $\beta$ abundance among healthy and degrading arbuscules can only be determined for individual fluorophores. 


\section{DISCUSSION}

To study how plants create a specialized symbiotic host-microbe interface that is distinguishable from the plasma membrane, we focused on t-SNAREs involved in exocytosis. We have shown that multiple syntaxins are expressed in arbuscular cells in Medicago. Of these syntaxins, only MtSYP132a is transcriptionally co-regulated with symbiotic interface formation and is essential for the formation of a stable host-microbe interface in both AM and rhizobial endosymbioses. This indicates that SYP132 allows a symbiosis specific regulation of exocytosis. Thus, there is specificity in the SNAREs that are used to form an interface, which indicates that besides the timing of expression of interface components, also the use of differently regulated trafficking pathways contributes to the specialization of the interface membrane. In most dicots, two spliceforms of SYP13II are present that localize differently to functional and degrading arbuscule branches. This suggests that the two spliceforms of SYP13II may facilitate exocytosis at different functional domains.

The existence of a dedicated subset of SNAREs to mediate traffic to the interface suggests that they are indeed key players in the regulation of traffic to the interface. Our initial hypothesis was that $t$-SNAREs determine the sub-cellular location of vesicle fusion and that different syntaxins could, for example, distinguish the interface from the membrane surrounding the arbuscule trunk or plasma membrane. However, we show here that all syntaxins that are (dominantly) expressed in arbuscule cells localize to both the plasma membrane, arbuscular trunk as well as the arbuscule fine branches. Furthermore, we did not observe a preferential localization of syntaxins at the tips of arbuscule fine branches, which indicates that t-SNAREs are unlikely to be the vectors that determine where vesicle fusion takes place. MtSYP122 was more abundant on the trunk domain of the arbuscule. The lower abundance of SYP122 on the peri-arbuscular membrane surrounding the fine branches correlates with the reduction of SYP122 expression in arbuscular cells compared to uninfected cortex cells (Fig. 2c). This is in line with the conclusion of Pumplin et al. (Pumplin et al., 2012) that the subcellular localization of proteins in arbuscular cells partially depends on the timing of their expression. The proteins encoded by the two spliceforms of SYP132 labelled the plasma membrane and peri-arbuscular membrane at equal levels in this study. However, it should be noted that the regulation by alternative splicing was bypassed in this experiment. Given their observed expression pattern, it is likely that SYP132 $\beta$ abundance in the natural situation is higher at the plasma membrane, while SYP132a is the dominant isoform that is present on the peri-arbuscular membrane. Thus, although t-SNAREs may not be the determinants of the location of vesicle fusion, their abundance at subcellular locations is variable. This suggests that each t-SNAREs may differentially affect the efficiency of vesicle fusion at different locations. The exact location of vesicle fusion is likely to be regulated by protein complexes such as the exocyst of which components accumulate at the tips of growing arbuscule branches (Genre et al., 
2012; Zhang et al., 2015b), in combination with remodelling of the cytoskeleton (Ivanov \& Harrison, 2014). EXO70i, an exocyst subunit that is dedicated to symbiosis, is not the only EXO70 subunit expressed in arbuscular cells, and is not essential to form the arbuscule trunk domain (Zhang et al., 2015b). This suggests that different EXO70 family members mediate vesicle fusion at different membrane compartments. Specific SNARE proteins could work together with distinct exocyst complexes to provide the specificity in secretory events at the different locations (Dubuke et al., 2015).

The reduction of arbuscules to small clumps after knock-down of SYP132a indicates that SYP132a is required for the formation or the maintenance of arbuscules. This strongly suggests that SYP132a controls the delivery of components that are required for arbuscule formation/maintenance. Likely candidates for such components are nutrient transporters such as the phosphate transporter PT4 and/or the essential half-ABC transporters (STR1/2), which when mutated also lead to premature collapse of the arbuscules (Javot et al., 2007; Zhang et al., 2010a). Apparently, the presence of SYP121 and SYP122 at the arbuscules is not sufficient to sustain the interface. This is striking since especially SYP121 is present at equal levels as SYP132a in arbuscular cells. This indicates that SYP121 controls different exocytosis events compared to SYP132a. At present we cannot rule out a role for the SYP12 orthologs in arbuscule development as there might be genetic redundancy among the three SYP12 members, as has been reported for SYP12 genes in Arabidopsis (Assaad et al., 2004). The observation that arbuscule formation in SYP132a RNAi roots can be complemented with SYP132 $\beta$ shows that the latter isoform has maintained the ability to function in interface formation. So in arbuscule forming cells they most likely mediate the fusion of the same vesicles, in line with their near-identical SNARE domains.

Our phylogenetic analysis indicates that SYP132a is the ancestral isoform and SYP132 $\beta$ evolved later. In this light, it is somewhat odd that Medicago SYP132 $\beta$ acquired an essential role in development, whereas it was lost in all analysed dicot AM non-hosts as well as in Cucumis and Vitis. The seemingly different role of SYP $13 \| \beta$ in different plant species may be explained by the observation that the evolution of SYP13॥ $\beta$ created a chimera between SYP13Ila and SYP13I, likely involving an unequal crossover event (Fig. 5). Therefore, the new properties of SYP13॥ $\beta$ may be identical or similar to the non-symbiotic SYP13I. This allows SYP13II $\beta$ to take over the role of SYP13I in plant development. In this scenario, the requirement for SYP13॥ 3 in certain plant species might merely reflect a low expression of SYP13I. Furthermore, if SYP13II $\beta$ is similar to SYP13I, its evolution may have provided a way to replace SYP13I with a similar protein that can be precisely inversely regulated to SYP13lla by alternative splicing.

Why do plants replace SYP132 $\beta$ during host-microbe interface formation with SYP132a, a near-identical protein, when also SYP132 $\beta$ is sufficient to form an interface? Based on our localization studies we postulate that the use of SYP132a allows the plant to restrict 
exocytosis to functional arbuscule branches. In partially collapsing arbuscules SYP132 $\beta$ preferentially labels the degrading parts of arbuscules, whereas SYP132a occurs mostly on the functional branches. The difference in SNARE abundance between the plasma membrane, arbuscule trunk and peri-arbuscular membrane could be attributed to the timing of expression. However, the observed difference in localization between the two spliceforms, when both are expressed from the same promoter, reveals that their subcellular location is differentially regulated at a post-translational level when arbuscule degradation is initiated. From our data, we cannot distinguish between a difference in delivery or turnover of the two spliceforms at functional and degrading arbuscule domains. A specific localization to functional arbuscule domains has also been reported for the phosphate transporters PT4 (Harrison et al., 2002) in Medicago and PT11 in rice (Kobae et al., 2014). Therefore, an intriguing hypothesis is that the turnover or delivery of SYP132a is directly linked to the symbiotic nutrient exchange across the membrane. This implicates that only arbuscules that deliver nutrients are maintained by the plant, as previously suggested by Javot and colleagues (2007), by SYP132a mediated delivery of nutrient transporters that is restricted to functional branches. This could act as a molecular sanctioning mechanism where carbon delivery to the fungus becomes dependent on nutrient delivery to the plant (Kiers et al., 2011). In this scenario, the difference between the two isoforms is not their ability to mediate the fusion of different vesicles, but their different dependence on nutrient exchange. The inverse transcriptional regulation of the two spliceforms by alternative splicing may then be crucial to avoid interference of SYP132 $\beta$ while the SYP132a-dependent sanctioning mechanism is active.

In conclusion, our results indicate that the regulation of vesicle traffic to the arbuscule during its formation and degradation is specialized and involves distinct SNAREs. The timing of gene expression relative to the moment of re-direction of exocytosis from the plasma membrane to the arbuscule is crucial to create the specific composition of the peri-arbuscular membrane (Pumplin et al., 2012). In addition, we have shown that this re-direction involves specific SNAREs, which implies that selectivity of exocytosis pathways may also contribute to the unique composition of the host-microbe interface. SYP132a is a symbiosis-dedicated regulator of exocytosis that has been co-opted by rhizobia to form a host microbe interface. This is in line with the co-option of the symbiotic signalling cascade and AM-symbiosis dedicated v-SNAREs in rhizobium symbiosis (Parniske, 2000; Ivanov et al., 2012). In AM symbiosis, alternative splicing of SYP13II may link arbuscule maintenance to functionality, although the exact role of this alternative splicing remains enigmatic and a topic for further study. 


\section{MATERIALS AND METHODS}

\section{Phylogenetic analysis}

The proteins sequence of all Arabidopsis exocytosis related t-SNAREs (Sanderfoot, 2007) were retrieved from the Arabidopsis information resource (TAIR) database. We identified their homologs in other plant species by BLASTing the genomes listed in Tables S1. For the SYP13 phylogeny, the SYP13 identity of all proteins was confirmed by their clustering with Arabidopsis and Medicago SYP13 proteins in a tree that contains all Arabidopsis and Medicago SYP1 proteins. Multiple sequence alignments were produced using the ClustalW algorithm in MEGA 6.0 (Tamura et al., 2013). From the alignment we reconstructed Neighbor Joining and Maximum likelihood trees, with 1,000 bootstrap replicates to assess clade support using MEGA 6.0. To generate consensus sequences, the aligned sequences of the last exon of SYP13 proteins were used as input sequence in WebLogo 3 , version 3.4 (http://weblogo.threeplusone.com/) (Crooks et al., 2004).

\section{Plant growth, transformation and inoculation}

Medicago truncatula Jemalong A17 was used in all experiments. For transformation, Agrobacterium rhizogenes MSU440 was used according to (Limpens et al., 2004). For nodulation assays, plants were transferred to perlite saturated with Färhaeus medium without $\mathrm{Ca}\left(\mathrm{NO}_{3}\right)_{2}$ and grown at $21^{\circ} \mathrm{C}$ at a $16 / 8 \mathrm{~h}$ light/dark regime. After three days, plants were inoculated with Sinorhizobium melilotii 2011 and grown for 2 weeks. For mycorrhization assays, plants were transferred to pots containing a 5:3 (v/v) ratio mix of expanded clay and sand, saturated with modified Hoagland medium ( $5 \mathrm{mM} \mathrm{MgSO}_{4^{\prime}} 2.5 \mathrm{mM} \mathrm{Ca}\left(\mathrm{NO}_{3}\right)_{2^{\prime}}$ $2.5 \mathrm{mM} \mathrm{KNO}_{3^{\prime}} 2 \mathrm{mM} \mathrm{NH}_{4} \mathrm{NO}_{3^{\prime}}, 500 \mu \mathrm{M}$ MES, $50 \mu \mathrm{M} \mathrm{NaFeEDTA}, 20 \mu \mathrm{M} \mathrm{KH}_{2} \mathrm{PO}_{4^{\prime}}, 12,5 \mu \mathrm{M} \mathrm{HCl}$, $10 \mu \mathrm{M} \mathrm{H}_{3} \mathrm{BO}_{3^{\prime}} 2 \mu \mathrm{M} \mathrm{MnCl}_{2^{\prime}} 1 \mu \mathrm{M} \mathrm{ZnSO}_{4^{\prime}} 0.5 \mu \mathrm{M} \mathrm{CuSO}_{4^{\prime}} 0.2 \mu \mathrm{M} \mathrm{Na}_{2} \mathrm{MoO}_{4^{\prime}} 0.2 \mu \mathrm{M} \mathrm{CoCl}_{2^{\prime}} \mathrm{pH}$ 6.1). Plants were either co-cultivated with Rhizophagus irregularis infected Allium schoenoprasum (used in initial analysis of SYP132a RNAi), or inoculated with dried Rhizophagus irregularis infected maize roots obtained from Plant Health Cure (http://www.phc.eu). Plants were grown for four weeks at $21^{\circ} \mathrm{C}$ at a $16 / 8 \mathrm{~h}$ light/dark regime.

\section{Laser capture microdissection}

Roots of mycorrhized Medicago plants and uninfected control plants were harvested and fixed in Farmer's fixative (75\% ethanol, 25\% acetic acid) substituted with 0,01\% Chlorazol Black $E$ to stain AM fungi, and vacuum infiltrated for 30 minutes on ice. Then, the roots were incubated in Farmer's fixative for 16 hours at $4^{\circ} \mathrm{C}$ on a spinning wheel. After fixation, the roots were dehydrated in an ethanol dehydration series $(80 \%, 85 \%, 90 \%, 95 \% 30$ minutes each followed by $100 \%$, overnight) Steedman wax was prepared by mixing $90 \%$ polyethylene glycol400 distearate and $10 \% 1$-hexadecanol at $65{ }^{\circ} \mathrm{C}$. Steedman wax was infiltrated by incubating the roots in $50 \%$ Steedman wax and $50 \%$ ethanol for two hours 
at $38{ }^{\circ} \mathrm{C}$, followed by 3 incubations in $100 \%$ Steedman wax for 2 hours at $38{ }^{\circ} \mathrm{C}$. Finally, the samples were transferred to room temperature to allow the wax to solidify. Solidified blocks of Steedman wax were cut into $20 \mu \mathrm{m}$ thick sections using a microtome, and transferred to PEN-membrane slides (Leica). Arbuscule containing cells and uninfected cortical cells were harvested using a Leica LMD7000 laser capture microdissection microscope. RNA was isolated using a RNeasy micro kit (Qiagen). cDNA was synthesized using the iScript Select cDNA synthesis kit (Bio-Rad), using random hexamer primers. Finally, the amplicons of all genes to be tested were pre-amplified using the SsoAdvanced PreAmp Supermix (Bio-Rad) before quantitative real-time (qRT)-PCR.

\section{RNA isolation and qRT-PCR}

RNA was isolated from plant tissue using the EZNA Plant RNA mini kit (omega). cDNA was synthesized from $1 \mu \mathrm{g}$ of RNA using the iScript cDNA synthesis kit (BioRad). Equal amounts of cDNA were used for qPCR using SYBR green supermix (Bio-Rad) in a Bio-Rad CFX connect real-time system qPCR machine. Gene expression levels were determined using gene specific primers listed in table S2. The gene expression was normalized using Actin2 as reference gene, or Actin2, Ubiquitin 10 and UB9 in case of the laser microdissected samples. To allow the detection of relative expression levels, the amplification efficiency for each primer combination was determined using standard curves based on a dilution series of cDNA.

\section{Plasmid construction}

The coding sequences of MtSYP132a, $-\beta$, MtSYP121 and MtSYP122, and short fragments for RNAi were amplified by PCR using primers listed in table $S 2$, of which the forward primers contain a CACC adapter for TOPO cloning. To create codon scrambled versions of SYP132, the last part of the cds of SYP132a, starting from a unique EcoRI site, was replaced using restriction/digestion with de novo synthesized gene fragments (gBlocks, Integrated DNA technologies), encoding the last part of either SYP132 $a$ or $-\beta$, flanked by an EcoRI site and an Ascl site (Fig S1). The 2 kb upstream promoter regions of MtSYP132, MtSYP121 and MtSYP122, and the PT4 promoter as reported by Pumplin et al. (Pumplin et al., 2012) were amplified by PCR using primers listed in table S2, of which the forward primers contain a CACC adapter as well as a HindIII restriction site. The PCR fragments were cloned into a pENTR/D-TOPO entry vector using TOPO cloning (Invitrogen). The promoters were subsequently cloned into pENTR4-1 entry vectors containing GFP or mCherry using HindIII-Ascl restriction/ligation.

For generation of RNAi constructs, the short gene fragments were recombined into a pK7GWIWG2(II) binary vector containing a dsRed selection marker (Limpens et al., 2004), using Gateway technology (Invitrogen). Codon scrambled expression cassettes as well as GFP and mCherry fusions were created using multisite Gateway technology (Invitrogen) 
in the pKGW-RR-MGW destination vector (Ovchinnikova et al., 2011). For cloning multiple expression cassettes into one binary vector, single expression cassettes were constructed as described above, and subsequently amplified by PCR using primers listed in table S2, adding Spel and Swal sites (GFP fusion constructs) or Swal and Apal sites (mCherry fusion constructs). Then both fragments were cloned into one pKGW binary vector by Spel-SwalApal restriction and ligation. To combine codon scrambled expression cassettes with RNAi vectors, the expression cassettes were amplified adding Apal and Eco81I sites, and cloned into the RNAi vectors by restriction and ligation.

\section{Mycorrhizal staining and quantification of mycorrhization}

For quantification of mycorrhization, roots were incubated in $10 \%(\mathrm{w} / \mathrm{v}) \mathrm{KOH}$ at $98{ }^{\circ} \mathrm{C}$ for 10 minutes. Then roots were washed 3 times with $5 \%$ acetic acid. After washing, the roots were stained in $5 \%$ ink in $5 \%$ acetic acid, for 2 minutes at $98{ }^{\circ} \mathrm{C}$. after staining the roots were destained in 5\% acetic acid, refreshing the destaining solution several times. For staining with WGA alexafluor 488 , roots were incubated in $10 \%(\mathrm{w} / \mathrm{v}) \mathrm{KOH}$ at $60{ }^{\circ} \mathrm{C}$ for 3 hours. Then, roots were washed 3 times in PBS $\left(150 \mathrm{mM} \mathrm{NaCl}, 10 \mathrm{mM} \mathrm{Na}{ }_{2} \mathrm{HPO}_{4^{\prime}} 1.8 \mathrm{mM}\right.$ $\mathrm{KH}_{2} \mathrm{PO}_{4^{\prime}} \mathrm{pH}$ 7.4), and incubated in $0.2 \mu \mathrm{g} / \mathrm{mL}$ WGA-Alexafluor 488 (Molecular Probes) in PBS at room temperature for 16 hours. For quantification of mycorrhization, roots were cut into $1 \mathrm{~cm}$ fragments, and the colonization and arbuscule abundance was scored and calculated according to Trouvelot et al. (Trouvelot et al., 1986).

\section{Plastic embedding and staining}

Nodules and roots were fixed in $5 \%$ glutaraldehyde in $0.1 \mathrm{M} \mathrm{PO}_{4}$ buffer $(\mathrm{pH} 7)$ for one hour at room temperature. Then, the samples were dehydrated in an ethanol dehydration series, followed by infiltration of Solution A (Technovit 7100 solution (Heraeus-Kulzer), 2,5\% PEG400, 1 pack/100 $\mathrm{ml}$ Hardener I). After infiltration, the plant material was embedded in polymerization solution (15ml solution A, $1 \mathrm{ml}$ Hardener II), and incubated overnight at room temperature. Polymerized blocks were then glued on holders for sectioning with Technovit 3040 (66\% powder, 33\% liquid), and cut into $6 \mu \mathrm{m}$ sections on a microtome (Leica 2035). Plastic sections were stained for 30 seconds in $0.05 \mathrm{M}$ Toluidine Blue and destained in water for 10 minutes.

\section{Microscopy}

For conventional light microscopy, a Leica DM5500 light microscope was used. For confocal imaging, a Zeiss 710 and Leica SP8 confocal microscopes were used. An excitation wavelength of $488 \mathrm{~nm}$ was used for GFP. An excitation wavelength of $543 \mathrm{~nm}$ was used for mCherry. Appropriate emission filter settings were used to separate the fluorophores used in each experiment. 


\section{Quantification of fluorescence}

To determine the ratio of fluorescence on functional and collapsing parts of the arbuscule, transects were drawn across at least 3 different locations of collapsing and functional arbuscules using ImageJ software. The average of fluorescence of each fluorophore was compared between the two subdomains for each image.

\section{Statistical analysis}

For all statistical analyses, SPSS statistics version 22 (IBM) was used. For quantification of nodule formation, root length, number of roots and mycorrhization, a Shapiro Wilk test was used to determine whether the data was normally distributed. Since this generally was not the case, the nonparametric Mann Whitney $U$ test was applied. The different fraction of NifH-GFP positive and NifH-GFP minus nodules was tested using a chi-Square test. qPCR data was analysed using the Students t-test.

\section{ACKNOWLEDGEMENTS}

We thank Ilias Gkikas for his contribution to the project and Dr. Robin van Velzen for help with the phylogenetic analysis. We thank Pius Floris of Plant Health Cure for supplying mycorrhizal inoculum. The authors declare no conflict of interest. R.H., T.B. and E.L. are supported by the European Research Council (ERC-2011-AdG294790). The Medicago truncatula tnt-1 insertion plants utilized in this research project, which are jointly owned by the Centre National De La Recherche Scientifique, were obtained from The Samuel Roberts Noble Foundation, Inc. and were created through research funded, in part, by a grant from the National Science Foundation, NSF-0703285.

\section{AUTHOR CONTRIBUTIONS}

R.H., T.B and E.L conceived and designed experiments. R.H., J.H., K.M., J.W and E.L performed experiments. R.H. and E.L analyzed the data. R.H., T.B. and E.L wrote the manuscript. 


\title{
Chapter 3
}

\section{SUPPLEMENTAL INFORMATION}

\begin{abstract}
$>$ SYP132 $\alpha$ scramble
actGAATTCCAGACTCTCAGACAAAGAATACAAGATGAATATCGTGAGGTTGTGGAGAGAAGAGTTATTACAG TTACGGGAACTAGACCAGATGATGAGACGATTGACCACCTGATAGAAACTGGAAACAGTGAGCAAATCTTCCA GCAGGCAATTCTTGAAGCAGGCCGAGGACAGGTCGTGAGCACTGTAGAAGAAATTCAGGAGAGACATGATGC TGTGAAAGAAATTGAGAAAAACTTCTTGATTTACATCAGATTTACCTTGACATGGCAGTGTTAGTTGAAGCTC AAGGAGAAATTITAGACAACATCGAAAGCCAGGTTGCAAACGCTACAGATCACGTGAAAAGCGGAAATGACG CTITGCACACAGCAAAGTCTTTACAGAAGAAaTCAAGAAAATGtATGATGATaGCAATTATTCTCGTCTTACTTA TTGCTATITTATTGTTCTTGGGGTAGTGAAgCCCTGGAAaAAAtgaGGCGCGCCtaa
\end{abstract}

\begin{abstract}
$>$ SYP132 $\beta$ scramble
actGAATTCCAGACTCTCAGACAAAGAATACAAGATGAATATCGTGAGGTTGTGGAGAGAAGAGTTATTACAG TIACGGGAACTAGACCAGATGATGAGACGATTGACCACCTGATAGAAACTGGAAACAGTGAGCAAATCTTCCA GCAGGCAATTCTTGAAGCAGGCCGAGGACAGGTCGTGAGCACTGTAGAAGAAATTCAGGAGAGACATGATGC TGTGAAAGAAATTGAGAAAAAACTTCTTGATTTACATCAGATTTACCTTGACATGGCAGTGTTAGTTGAAGCTC AAGGAGAAATTTTAGACAACATCGAAAGCCAGGTTAACAATGCTGTAGATCACGTGCAAAGAGGCACCACCG CTITACAATCTGCCAAGAAGCTCCAAAAGAACTCAAGAAAgTGGATGTGTATTGCTATAATTATCTTGCTTATTA TIGTAGCTATTATCGTTGTGGGAGTTCTTAAGCCATGGAAGTCCTCTtgaGGCGCGCCtaa
\end{abstract}

\section{Fig.S1 synthesized DNA fragments for cloning codon-scrambled constructs}

Gene fragments synthesized for cloning of codon scrambled constructs. Highlighted in grey is the sequence of the last exon of each spliceform that was codon scrambled by using the IDT codon optimization tool (http://eu.idtdna.com/CodonOpt). Highlighted in yellow and green are the EcoRI and Ascl site that were used to replace the original SYP132 sequence with these fragments.
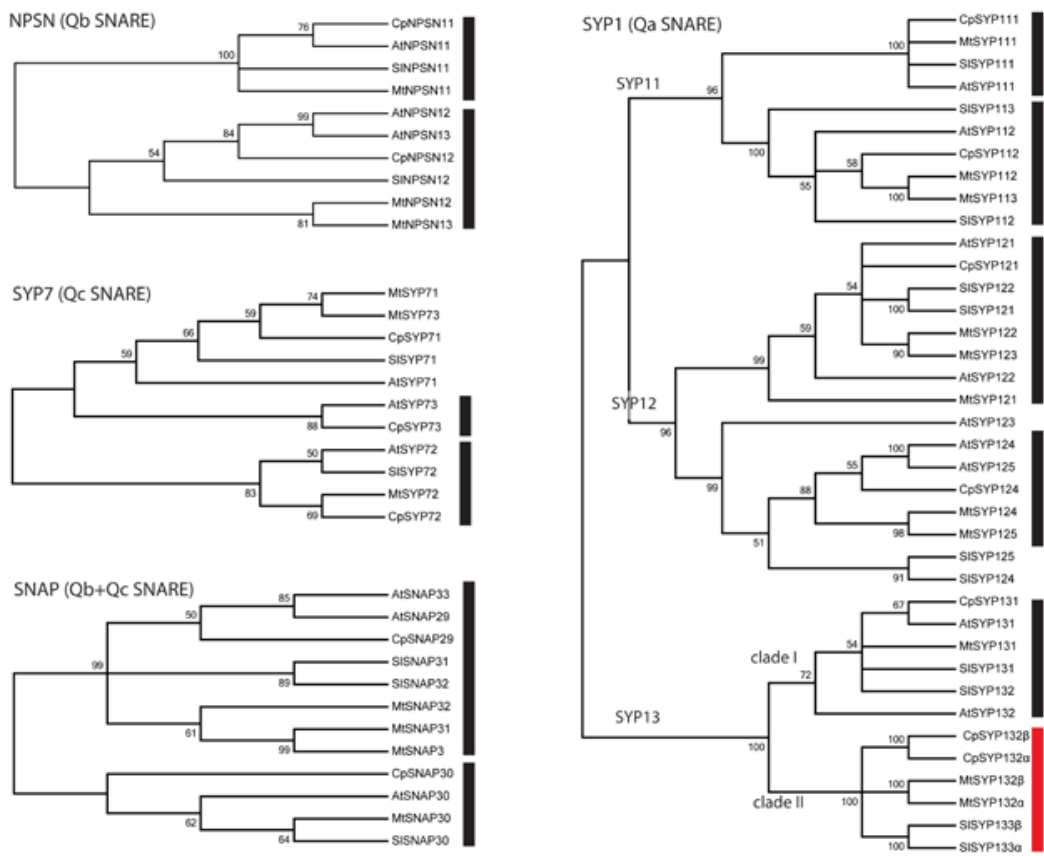

Fig. S2 Phylogenetic analysis of all exocytosis related t-SNAREs in different plant species.

Un-rooted Neighbour joining trees, showing the phylogeny of all exocytosis related t-SNARE families (Sanderfoot et al., 2007) of Arabidopsis (At), Medicago (Mt), papaya (Cp) and tomato (SI) (Suplemental table S1). Bars indicate orthology groups; a group of genes derived from a single ancestral gene that is likely to have conserved function in different species. For assigning orthology groups, we allowed intra-species gene duplications, and demanded a bootstrap support of at least 70. Black bars indicate orthology groups containing an Arabidopsis orthologs. The red bar indicates an orthology group (SYP13 cladell) that contains genes from Medicago, papaya and tomato, but lacks an Arabidopsis ortholog. 

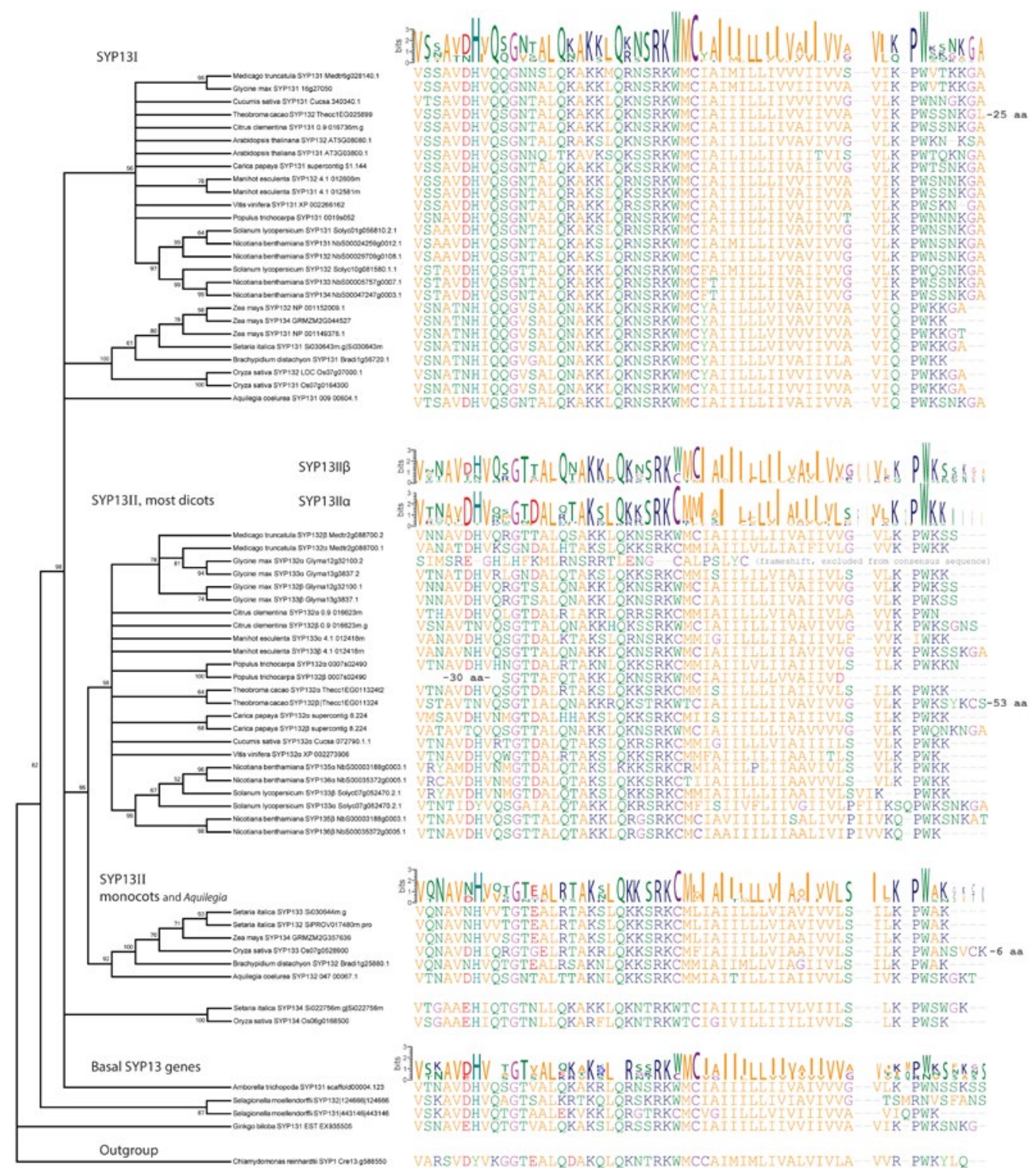

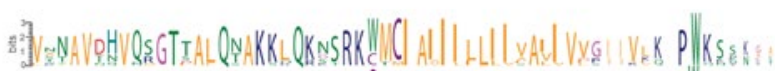

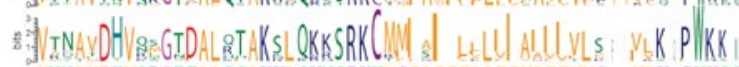

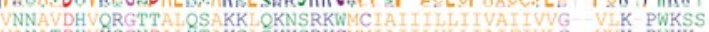

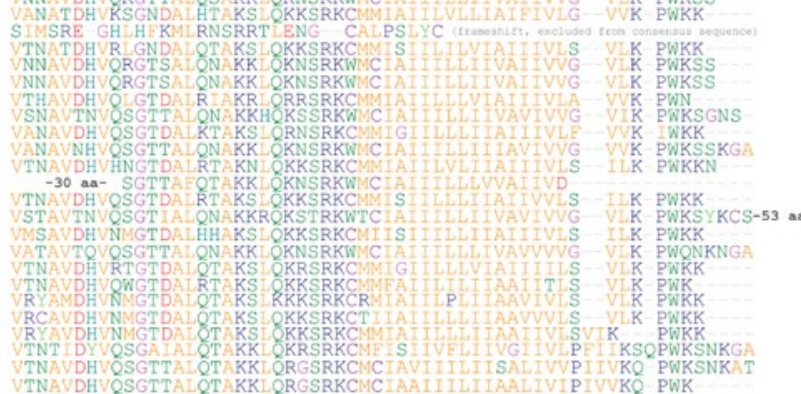

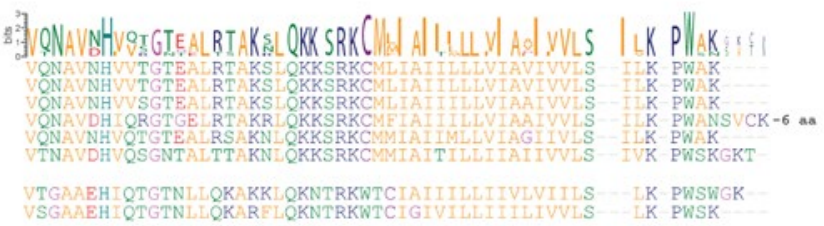
负

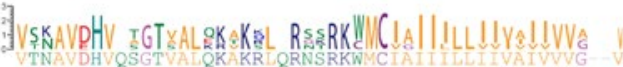

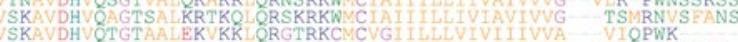

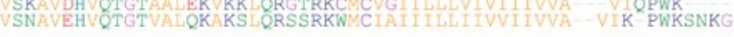
VARSVDYVKGGTEALQDAKQLQKNTRKWNCCAIMIMLIVALVIVLA VVR PWKYLQ

Fig. S3 Evolution of the SYP13 clade in land plants.

Maximum likelihood tree showing the phylogeny of SYP13 genes in a wide range of plant species. The full length amino acid sequence of all genes was used (Table S1). Chlamydomonas reinhardtii SYP1 was used as an outgroup. The amino acid sequence of the last exon of all transcripts is shown, on which the consensus sequence as shown in Figure 5 was based. 


\section{Chapter 3}

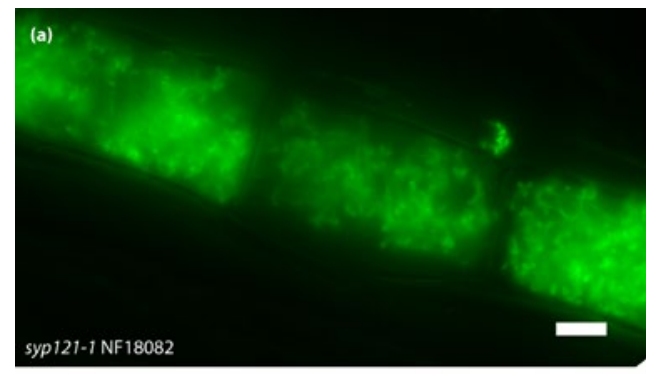

(d)
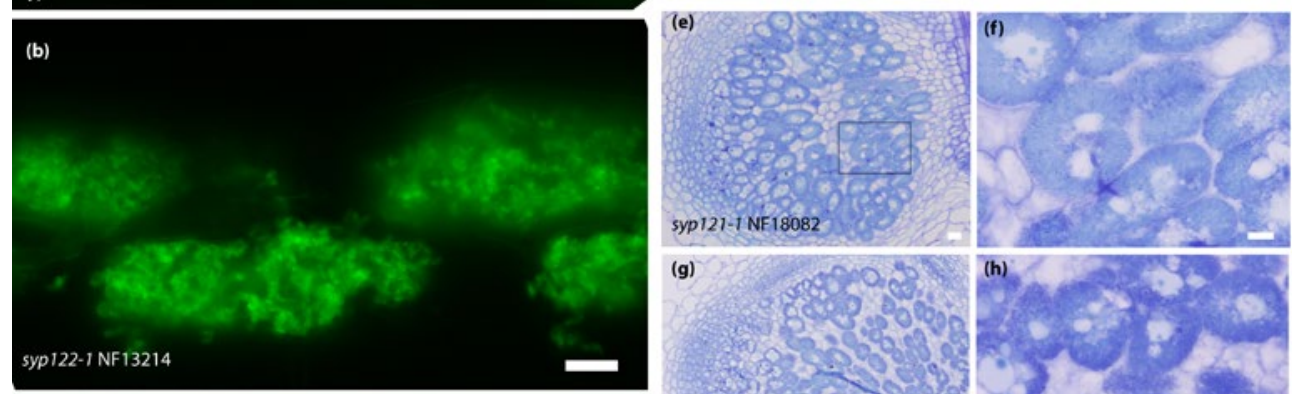

(g)
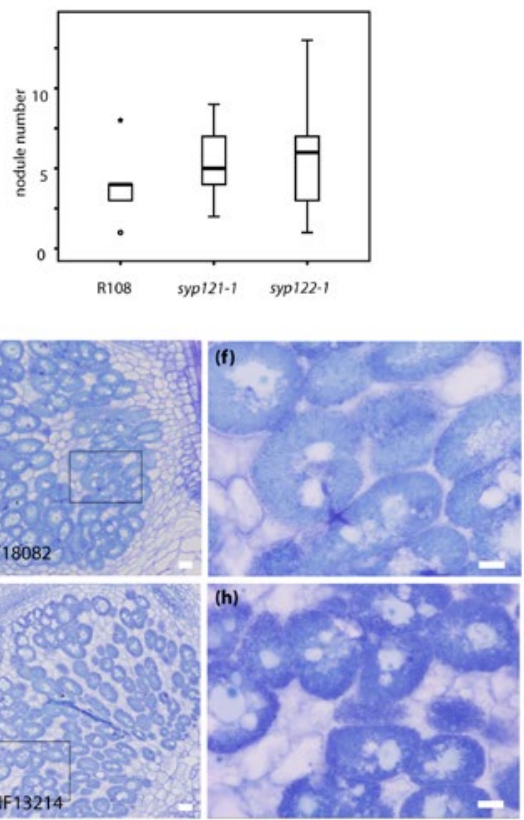

(c)
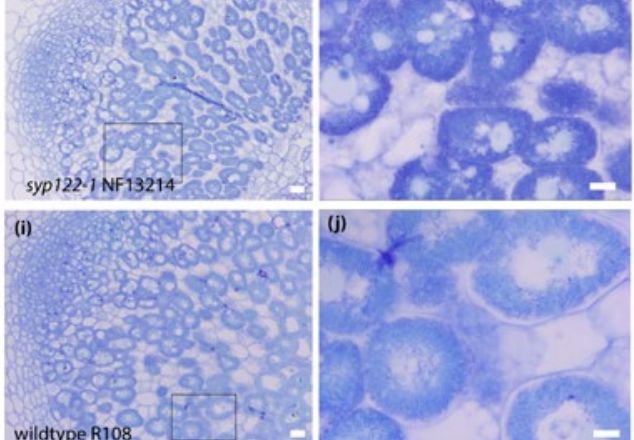

\section{Fig. S4 SYP121 and SYP122 are not required to form a host microbe interface.}

(a-c) Microscope images showing WGA-alexafluor 488 stained arbuscules in the syp121-1 mutant (a), the syp122-1 mutant, (b) and wildtype roots (c). scalebars are $10 \mu \mathrm{m}$. (d) Graph displaying the amount of nodules formed on wildtype, syp121-1 and syp122-1 roots. Differences are not significant (Mann-Whitney U test). (e-j) Semi-thin $(6 \mu \mathrm{m})$ section of a nodules (14 dpi) on syp121-1 mutant roots (e-f), syp122-1 mutant roots ( $g-h)$ and wildtype roots (i-j) scalebars are $10 \mu \mathrm{m}$. 
(a) number of transgenic roots per plant

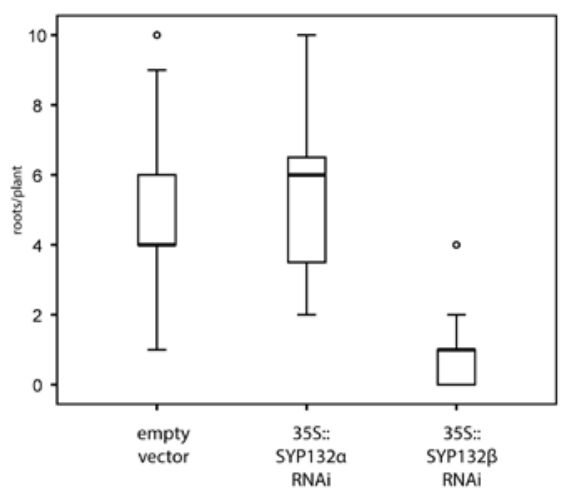

(c)

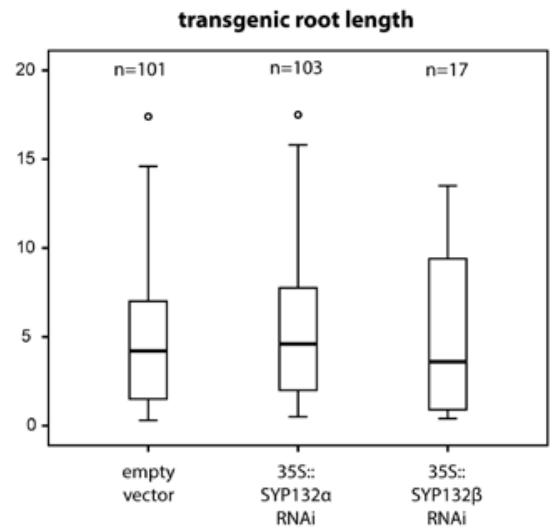

(b) gene expression in transgenic roots

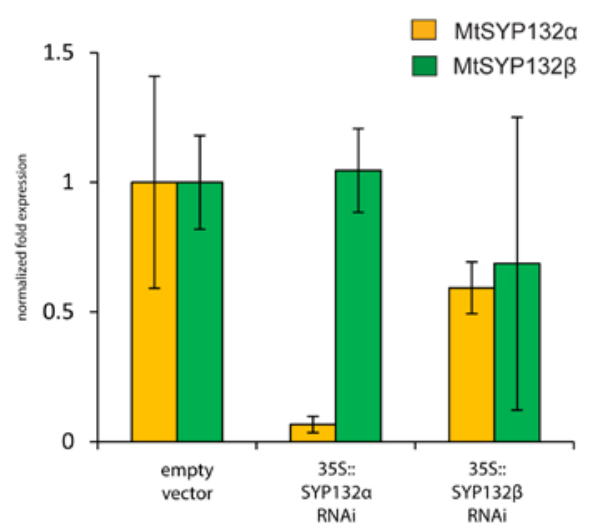

\section{Fig. S5 Non-symbiotic phenotype of SYP132 $\alpha$ and SYP132 $\beta$ RNAi}

(a) Box plot displaying the number of transgenic roots formed on plants transformed with a silencing construct targeting SYP132a or SYP132 $\beta$, compared to plants transformed with an empty vector. On plants transformed with the SYP132 $\beta$ silencing construct, significantly less roots were formed ( $n=20$ plants, Mann Whitney $U$ test, $p<0.01$ ). Differences between SYP132a RNAi and empty vector were not significant. The experiment has been carried out three times with similar results. (b) qRT-PCR showing the knockdown of SYP132a and SYP132 $\beta$ in transformed roots. Data are represented as the mean of 5 biological replicates \pm standard deviation. (c) Box plot displaying the root length of transgenic roots on plants transformed with the different silencing constructs. Differences are not significant (Mann Whitney $\mathrm{U}$ test). 


\section{Chapter 3}

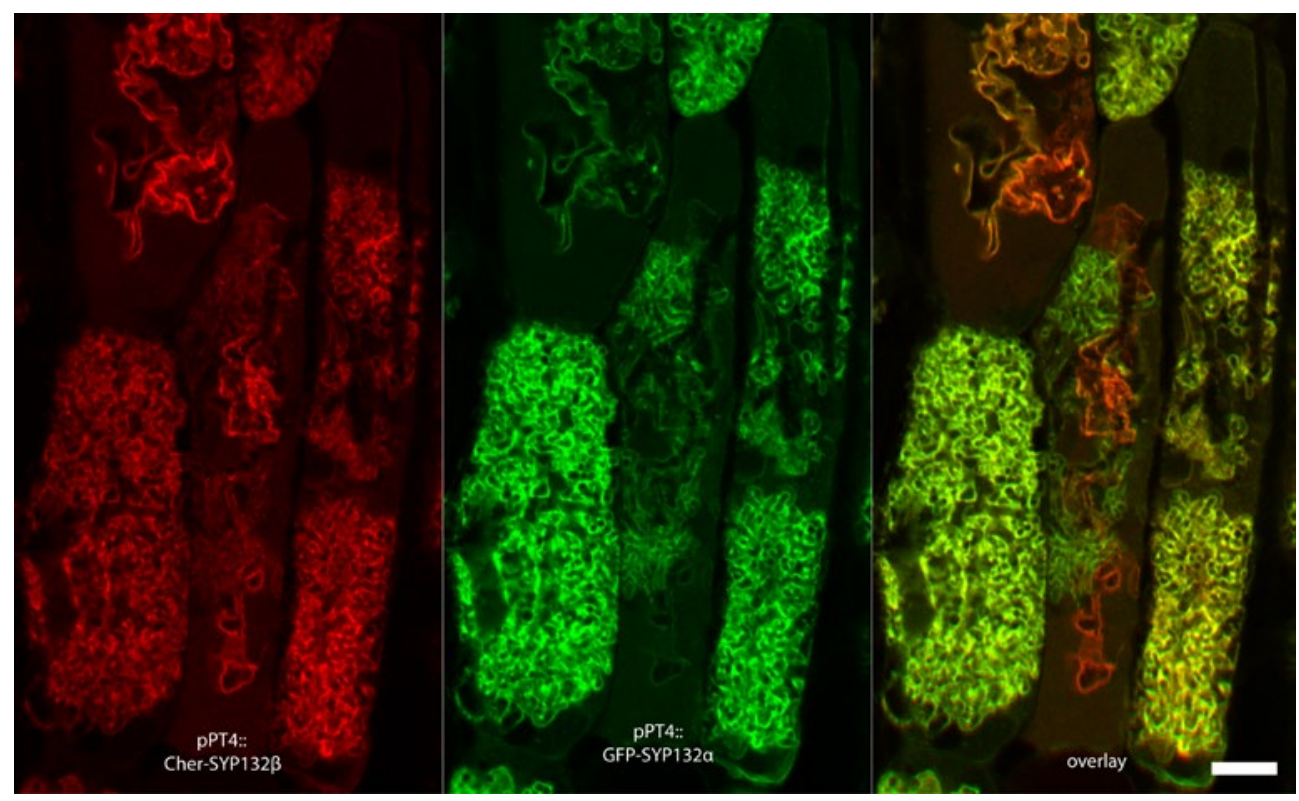

\section{Fig. S6 MtSYP132 $\alpha$ and - $\beta$ show different subcellular localization during arbuscule degeneration}

Double localization of GFP-labeled SYP132 $a$ and mCherry labeled SYP132 $\beta$ expressed from the PT4 promoter. The overview allows comparison between cells in different stages of arbuscule development. In cells harboring mature arbuscules, both spliceforms are present on the peri-arbuscular membrane. In cells harboring collapsing arbuscules, SYP132a has a clear preference for the functional part of the arbuscule, while SYP $132 \beta$ is preferentially localizing to the collapsed part of the arbuscule. In cells where the entire arbuscule is collapsed, leaving only a clump, both spliceforms are present on the clump, although the ratio is shifted to SYP132 $\beta$ compared to the healthy mature arbuscules.

\section{TableS1 Sequence information of t-SNAREs used in this study}

may be found online at onlinelibrary.wiley.com/store/10.1111/nph.13973/asset/supinfo/nph13973-sup-0002-TableS1.xlsx 


\section{A SYP13/l isoform controls host-microbe interface formation}

\section{Table S2}

primers used in this study

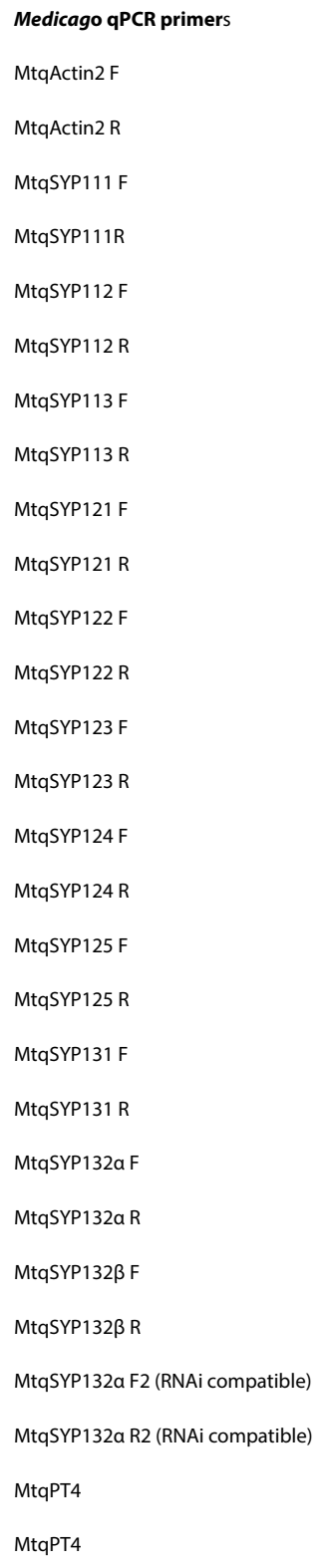

CAGATGTGGATCTCCAAGGGTGA

TGACTGAAATATGGCACAAGACTGAGA

GCTGTTACTAATGGCTTGCG

CATCATTTTCTGCCTAAGTCCC

CTACACTGTCACAGGCGAGG

CTCTGAATATCCATCGCAGC

ACCGAATGGATTCCGATATG

GCTTCGATTCGCAATGTTTG

AGAGAGACTGTTCAACGGCG

TCCAGAATTCTTCCCCTGC

TCGAAATGTCATCACCGAAC

GTGAAGGGTCTTGCTCCTCTC

CCCGTGAATGGACATGTTTT

CATAATCAATCAACCTTCACAGTAGC

AGGAAAACTCGAAGCATTGG

CTTCCCTAAACCACTAACCACTG

GTTCGAATGCCGCGAATAG

TCCCAATCCACTAACCACTG

ATTTGAGATCCCTCGTGGTC

CTATCCAAGCCAAGCTCTCC

ATGTGAAGTCGGGTAACGATG

CAATCATCATGCACTTCCTTG

CAGGTCAACAATGCAGTCG

CAATGCACATCCATTTTCGAG

GCTCAAGGAGAAATTTTAGACAAC

CAATCATCATGCACTTCCTTG

TCTCATGATCGGTGCTGTTC

TGTTTCGCGTTCCCTTCTAC

Nicotiana qPCR primers

NbqActin

NbqActin

NbqSYP135a F

NbqSYP135a R

NbqSYP136a F
NbqSYP136a R

NbqSYP135 $\beta$ F

NbqSYP135 $\beta$ R

NbqSYP136 $\beta \mathrm{F}$

NbqSYP136ß R

Plasmid construction

Coding sequence

MtcdsSYP132 F

MtcdsSYP132a R

MtcdsSYP132 $\beta R$

MtcdsSYP122 F

MtcdsSYP122 R

MtcdsPIP2.7 F

MtcdsPIP2.7 R

promoters

MtpSYP132a F

MtpSYP132a R

MtpSYP121 F

MtpSYP121 R

MtpSYP122 F

MtpSYP122 R

MtpPT4 F

MtpPT4 R

RNAicontructs

MtrnaisYP132a F

MtrnaiSYP132a R

MtrnaiSYP132 $\beta \mathrm{F}$

MtrnaiSYP132 $\beta$ R

Combining expression cassetes

Spel-Gateway F

Swal-Gateway R

Swal-Gateway F

Apal-Gateway R
TTGAGAACCGAGAGCACGAC

GCAAAGAAACTACAAAGGGGC

ATGATGGGGACAACTATTAAAGC

TGCAAAGAAACTACAGAGGGG

GGGGATGACTATTAAAGCCG

CACCATGAACGACCTTCTCACTG
GAGATACCATGAACCTTGTTAAACTG
TCAATCCTGCTCTAAGATGTTCC

CACCATGAATGATTTATTCTCCGGC

CTATTTTTTAAGGACAATAGGTAAAACTATGA

CACCCATTCAAATACTTATTTTGTTTT

GTTTGTGTTCTTGTGTTTGTGC

CACCAAGCTTCATTTATGGGAGTTTGAAGTTAGG

GAGAGTAAAACGGCAACAAGGA

CACCAAGCTTCTATTGAAGTCATACTCAGAGATG

AAAGAGTTTTGAAGTCAATGGATATTT

CACCAAGCTTCTGGACAACACAAAACTGA

CTTCTTGTTTATGTAAGAAAAAATAGAGAAGA

CACCAAGCTTGACTCGATCCACAACAAAGATT

AAAAAGGAAGACTCTCTCAAGTTGG

CACCAGGTGGCAAACGCAACTGA

GAGATACCATGAACCTTGTTAAACTG

CACCTCAACAATGCAGTCGATCA

CAAGGAAATAAGAAGCCATGA

TGACTAGTACGCCAAGCTATCAACTTTGT

GCATTTAAATCACGACGGCCAGTGAAT

TGATTTAAATACGCCAAGCTATCAACTTTGT

TCATAACGTGACTCCCTTAATTCTC 


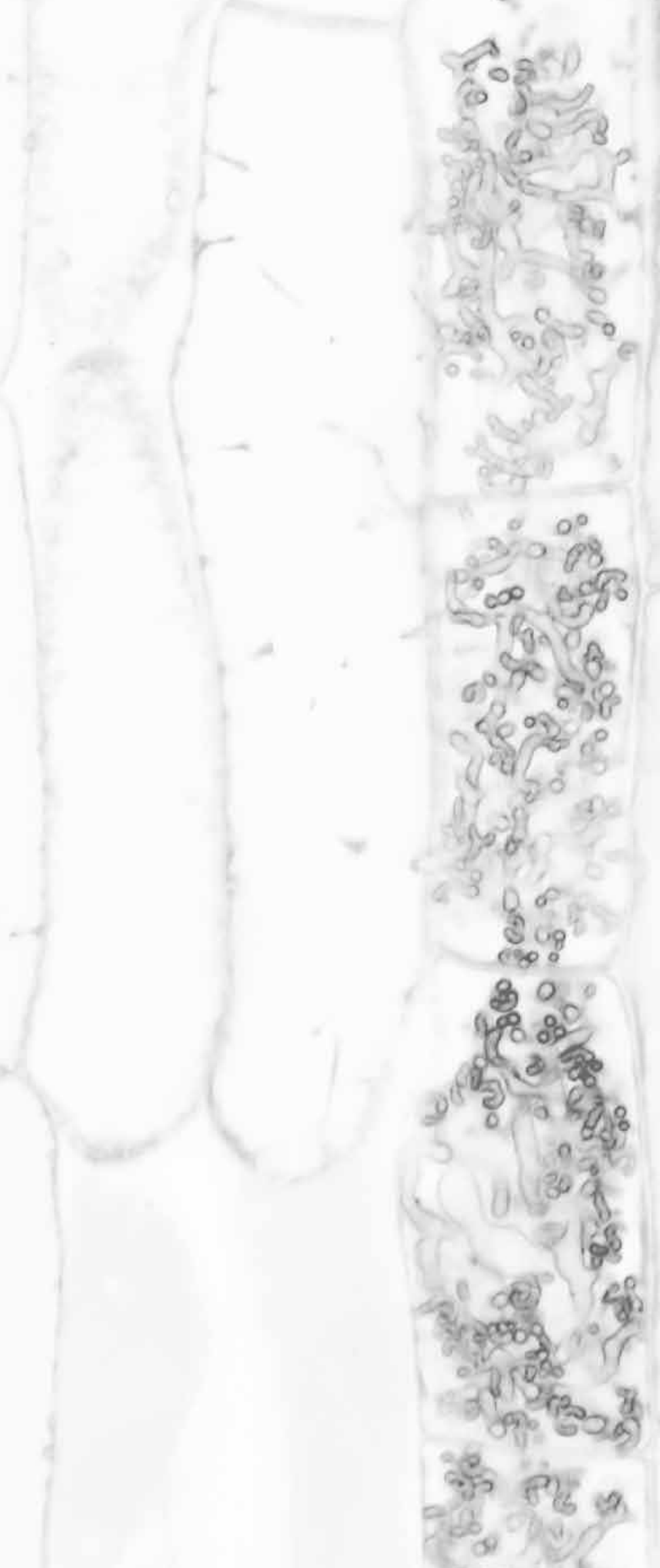




\section{Chapter 4}

Functional reduncancy among plant exocytotic SNARE proteins to form a symbiotic interface

Rik Huisman, Ton Bisseling and Erik Limpens

Directional growth and local responses of plant cells to their environment require the targeted delivery of vesicles and proteins to parts of the plasma membrane, creating a distinct subdomain. In plants, it is largely unknown how the polar localization of proteins to different membrane domains is controlled. An example of a specialized plasma membrane domain with a distinct protein composition is the peri-arbuscular membrane surrounding the arbuscules formed by arbuscular mycorrhiza (AM) fungi during AM symbiosis. Peri-arbuscular membrane formation depends on specific symbiosis dedicated v-SNAREs and t-SNAREs involved in exocytosis. This raised the hypothesis that cognate interaction of these SNAREs directs a subset of vesicles to the peri-arbuscular membrane. Therefore, we tested whether different SNAREs mark different plasma membrane domains in arbuscule containing cells. Furthermore, we tested whether the interactions between v-SNAREs and t-SNARE syntaxins on the peri-arbuscular membrane are selective. Finally, we systematically tested the ability of non-symbiotic SNAREs to replace the symbiotic SNAREs during symbiosis. Our data show that most secretory SNAREs localize to the peri-arbuscular membrane and can functionally replace the symbiotic SNAREs when expressed at sufficient levels to allow arbuscule formation. This functional redundancy is in line with the ability of all tested $v$ - and t-SNARE combinations to form SNARE complexes at the peri-arbuscular membrane, although the amount of v-SNAREs that co-purified with syntaxins was lowest for the combination of symbiotic SNAREs, suggesting a more strict regulation of SNARE complex stability. Our data show that the essential role of symbiotic SNAREs in arbuscule formation can be largely explained by their dominant expression level in arbuscule forming cells instead of specific protein properties. Together, our data suggest that the symbiotic SNAREs do not selectively interact to define a distinct vesicle trafficking pathway, but allow a more strict control over vesicle fusion during symbiosis, which is instrumental in forming a stable arbuscule. 


\section{INTRODUCTION}

The growth and maintenance of eukaryotic cells requires the continuous delivery of vesicles to the plasma membrane; exocytosis. The fusion of vesicles with their target membrane is driven by the interaction of vesicle SNAREs ( $v$-SNAREs or R SNAREs) on the vesicle with a complex of target membrane SNAREs (t-SNAREs) on the target membrane. t-SNAREs are classified into $\mathrm{Qa}, \mathrm{Qb}, \mathrm{Qc}$ and $\mathrm{Qb}+\mathrm{Qc}$ subclasses, based on the SNARE domains they contribute to the SNARE-complex. In plants, the number of SNAREs involved in exocytosis has expanded to be encoded by families of at least partially redundant proteins (Sanderfoot, 2007).

Different secretory SNARE family members are required for different biological processes (Chapter 6). This suggests that the evolutionary expansion of SNARE proteins allow the adaptation of exocytosis to these different biological processes. A key example of a biological process that depends on specific SNARE proteins is the formation of the peri-arbuscular membrane during the endosymbiotic interaction of plants with arbuscular mycorrhizal (AM) fungi. The peri-arbuscular membrane is a subdomain of the plasma membrane that surrounds the highly branched arbuscules in the plant cell, and has an unique protein composition to facilitate nutrient exchange between the two partners (Gutjahr \& Parniske, 2013). We have previously shown that the formation of arbuscules in Medicago truncatula (Medicago) depends on the two v-SNAREs MtVAMP721d and MtVAMP721e, and on the Qa-type t-SNARE (syntaxin) MtSYP132a (Ivanov et al., 2012; Huisman et al., 2016). Silencing of VAMP721d/e by RNAi results in a phenotype where the formation of arbuscular branches is almost completely blocked. RNAi of SYP132a results in small arbuscules that collapse prematurely. For simplicity, we will refer to these SNAREs as 'symbiotic' SNAREs while we will call other SNAREs'non-symbiotic', even though the latter ones may be involved in symbiosis as well.

The expansion of the number of secretory SNAREs in plants has been suggested to allow the presence of multiple exocytosis pathways in one cell (Sanderfoot, 2007; Kanazawa \& Ueda, 2017). We define an exocytosis pathway as the traffic and fusion of a distinct population of vesicles and associated cargo to the plasma membrane or subdomain. The use of separate exocytosis pathways would be a simple mechanism to create different plasma membrane subdomains with a distinguishable composition, like the peri-arbuscular membrane. Since the formation of the peri-arbuscular membrane depends on both a specialized $v$ - and t-SNARE, it is a particularly interesting model to study whether different SNAREs can mark distinct exocytosis pathways. Therefore, we questioned what the difference is between symbiotic SNAREs and their non-symbiotic paralogs, to find out whether they can define a specialized symbiotic exocytosis pathway that distinguishes the peri-arbuscular membrane from the plasma membrane. 
SNAREs are ideal candidates to define vesicle trafficking pathways. The idea that selective interaction between v-SNAREs and t-SNAREs encode specificity of vesicle fusion to the right membrane (sub)compartment is as old as the discovery of SNAREs themselves (Sollner et al., 1993). Indeed, different cellular compartments are labelled by unique sets of SNARE proteins, that control the fusion of vesicles to the proper compartment (Rothman, 1994; Mcnew et al., 2000). An example of the use of different exocytosis pathways to create specialized membrane domains can be found in animal cells: In mammalian polarized epithelial cells two plasma membrane domains with a distinct protein composition are present; an apical domain and a basolateral domain (Mostov et al., 2003). Trafficking of proteins to these domains is mediated by distinct populations of vesicles, which depend on distinct v-SNAREs (Martinez-Arca et al., 2003) and different t-SNAREs that are present at the two domains (Kreitzer et al., 2003).

Here, we studied to what extent the symbiotic SNAREs are different from their non-symbiotic family members and whether symbiotic SNAREs define a distinct symbiotic membrane trafficking pathway by studying their functional redundancy with their closest non-symbiotic paralogs, as well as with family members that are expressed in the same cell. Furthermore, we compared the localization and SNARE-interactions of symbiotic and non-symbiotic SNAREs in arbuscule cells. We showed that all tested SYP1 family proteins are able to complement the defect in arbuscule formation upon knockdown of SYP132a suggesting functional redundancy between the t-SNAREs. Also some of the non-symbiotic VAMP72 family proteins were able to complement the loss of symbiotic v-SNAREs VAMP721d/e. Surprisingly, although all combinations of SYPs and VAMPs showed interaction in arbuscule cells, the amount of v-SNAREs that co-purified with SYPs was lowest for the combination of symbiotic SNAREs. Together, our data suggest that the symbiotic SNAREs do not selectively interact to define a symbiotic vesicle trafficking pathway, but allow a more strict control over vesicle fusion during symbiosis, which is instrumental in forming a stable arbuscule. 


\section{RESULTS}

\section{The Qa- and R-SNARE repertoire of arbuscule containing cells.}

To guide functional comparisons we first investigated the phylogenetic relationship of the symbiotic SNAREs with other secretory SNAREs, and determined which family members are expressed in arbuscule cells. The individual SNARE genes were divided into orthogroups that are shared and conserved among a wide range of plant species (Fig 1a). This classification allows comparison of our data with earlier studies on SNARE interactions in other plant species (Table 1). Furthermore, comparing SNARE genes from separate orthogroups is most likely to reveal specialization, since proteins from different orthogroups are more likely to be functionally different than proteins from within one orthogroup. Finally, the distribution of orthogroups among different clades reveals the evolutionary trajectory of the symbiotic SNAREs, indicating when they evolved.

The vast expansion of the number of syntaxins occurs at the base of the angiosperms: Whereas we found only two conserved orthogroups in the gymnosperms, this number increases to 6 orthogroups in angiosperms, including SYP13/I that we linked to symbiosis earlier (Chapter 3). Evolution of the latter orthogroup is strictly linked to the ability of plant species to interact with AM fungi: It is conserved in all analysed AM host plant species $(16 / 16)$, and lost in all analysed plant species that independently lost the ability to interact with AM fungi (6/6). In the dicot lineage, SYP13/l is spliced into two different transcripts encoding SYP13lla and SYP13॥ $\beta$ that are at least partially functionally redundant (Chapter 3).

Within the VAMP72 family, 4 orthogroups can be found. Most individual VAMP genes of both Arabidopsis and Medicago are the result of independent and recent expansions within the VAMP72/ orthogroup. The symbiotic MtVAMP721d and MtVAMP721e genes form a clear group together with other VAMP genes from dicots, embedded within the VAMP72I orthogroup. We named this group VAMP72II. VAMP721/l does not contain monocot genes, nor genes from Aquilegia coerulea, the most basal sequenced eudicot. Thus, the symbiotic VAMPs likely evolved at the base of the dicot lineage, after the split of $A$. coeurulea. The conservation of VAMP72/l is largely, but not strictly, correlated to the ability of plants to host AM fungi. In particular, it is conserved in 1 out of 5 of the analyzed dicot AM non-host plants (Striga hermonthica), whereas it is lost in 2 out of 10 analysed dicot AM host plants (Manihot esculenta and Carica papaya).

To get an accurate overview of the relative expression of symbiotic SNAREs and their non-symbiotic paralogs in arbuscule cells, we isolated RNA specifically from arbuscule containing cells by making use of laser microdissection. Next, digital droplet PCR (ddPCR) was used to measure the absolute levels of transcripts of the different SNAREs. We limited our analysis to the genes that showed expression in this cell-type based on previous 
A
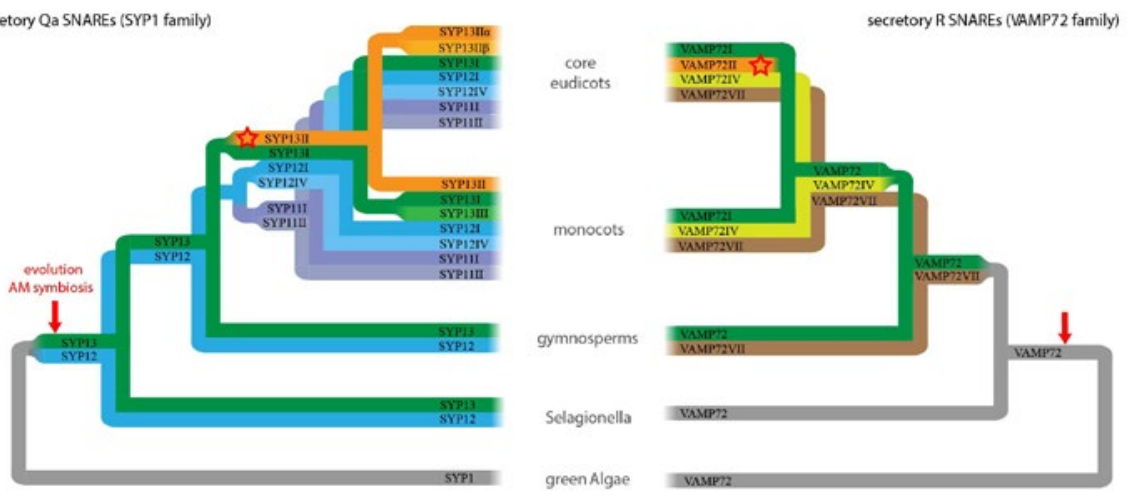

B
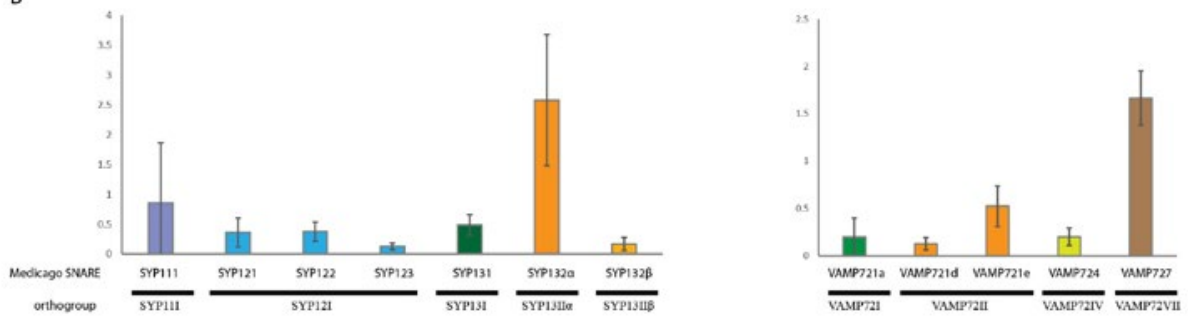

Figure 1. The SNARE repertoire of arbuscule containing cells.

A. Schematic phylogenetic trees showing the evolution of the exocytosis related SNARE families SYP1 and VAMP72. The trees are showing conserved orthogroups instead of individual genes, indicated by the use of roman numbers. Orthogroups were named following Sanderfoot 2007. Both schematic trees are based on the actual phylogenetic trees shown in supp. Figures 1 and 2. B. digital droplet PCR on CDNA from arbuscule containing cells. only SYP1 and VAMP72 genes that are expressed in arbuscule cells are shown. The copy number of each gene was normalised against the average copy number of all tested genes in each cDNA. Error bars represent standard deviation of three biological replicates.

qPCR analyses (Huisman et al., 2016). This showed that multiple SNAREs are expressed in arbuscule-containing cells. In these cells, MtSYP132a is clearly the dominant syntaxin (Fig 1b). VAMP727 is the most highly abundant v-SNARE transcript, followed by the symbiotic VAMP721e (Fig 1b).

Based on their phylogeny and expression in arbuscule containing cells, we selected SNARE proteins to be studied in more detail in a functional comparison. We selected the highest expressed symbiotic VAMP VAMP721e, along with the highest expressed gene of all other orthogroups; VAMP721a, VAMP724 and VAMP727. We selected both spliceforms of the symbiotic SYP13II; SYP132a and SYP132 $\beta$, their closest non-symbiotic paralog SYP131, and the more distantly related SYP121, all of which are expressed in arbuscule cells.(Fig 1b). 


\section{Most exocytosis related SNAREs localize to the peri-arbuscular membrane.}

We first investigated whether the selected SNARE proteins localize to the peri-arbuscular membrane (PAM). Therefore, we expressed the different SNAREs fused to GFP from the Medicago PT4 promoter that is highly and exclusively active in arbuscule cells (Pumplin \& Harrison, 2009), and determined their localization by confocal microscopy. All syntaxins localized to the peri-arbuscular membrane (Fig 2a-d), as shown earlier for SYP132a, SYP132 $\beta$ and SYP121 when expressed from their native promoter (Chapter 3). Even though v-SNAREs function on vesicles, overexpression typically results in their accumulation on the target membrane. VAMP721a, VAMP721e and VAMP724 accumulated on the peri-arbuscular membrane (Fig 2e-g). In contrast VAMP727 accumulated in punctuate intracellular structures, as well as on the tonoplast (Fig $2 \mathrm{~h}$ ).
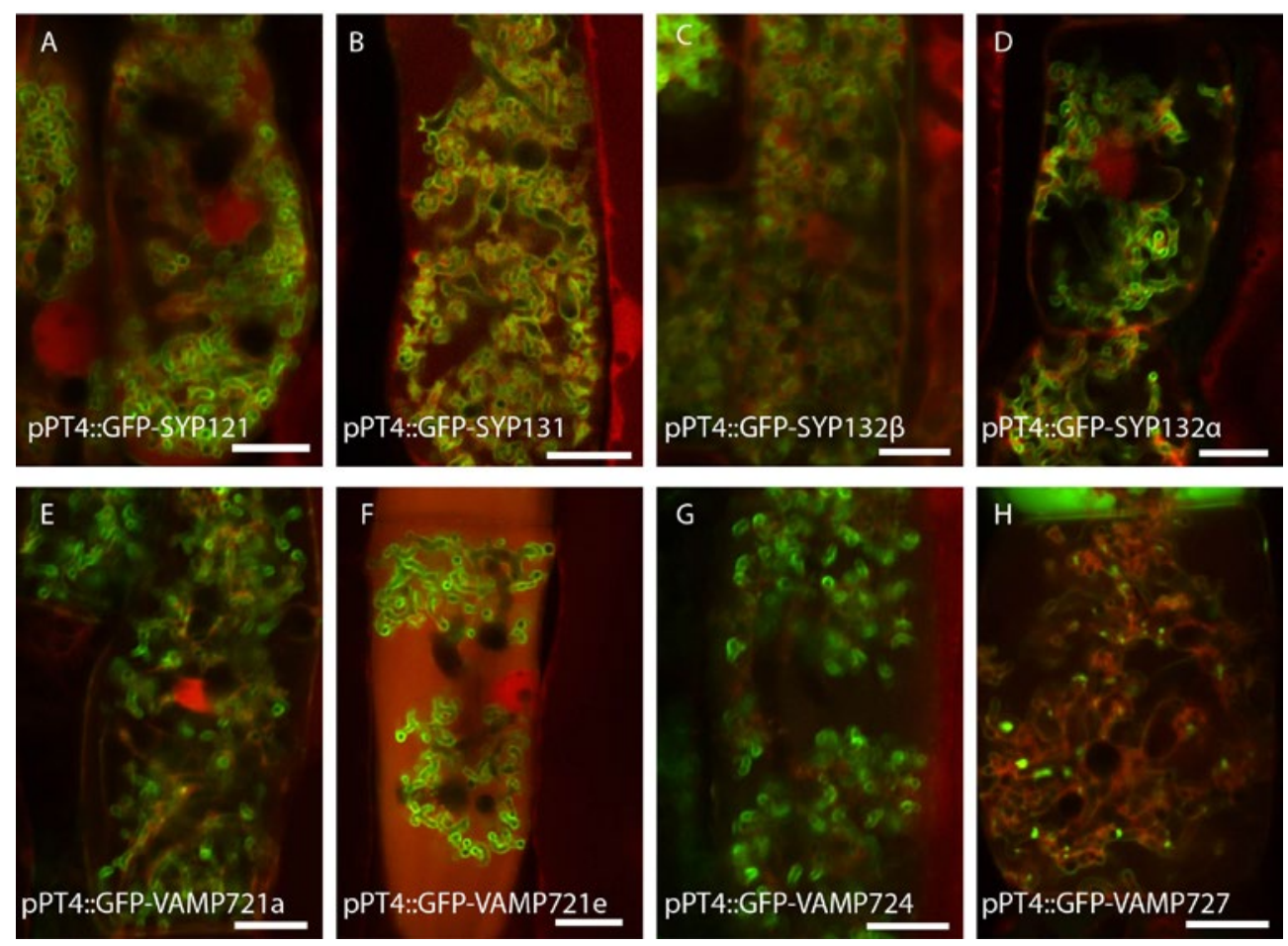

Figure 2. Intracellular localization of exocytosis related SNAREs

Intracellular localization of syntaxins (A-D) and VAMPs (E-H) in arbuscule containing cells. Different SNAREs fused to GFP were expressed from the arbuscule specific PT4 promoter, combined with dsRed that marks the cytoplasm and nucleus. Scalebars are $10 \mu \mathrm{m}$. 


\section{Interaction between v- and t-SNAREs}

Next, we tested whether there is specificity in SNARE interactions at the PAM, by using a co-immunoprecipitation (co-IP) approach. Therefore, we expressed combinations of GFP-labelled syntaxins and triple HA-tag labelled VAMPs from the PT4 promoter in mycorrhized Medicago roots. The GFP labelled syntaxins were pulled down from root extracts, and the co-IP of the VAMPs was determined by western blot using an antibody against the HA-tag (Fig 3). For all combinations of syntaxins and VAMPs, the anti-GFP blot revealed two bands of around $63 \mathrm{kDa}$ and $25 \mathrm{kDa}$, representing the SYP-GFP fusion protein and free GFP respectively. The bands in the IP lanes were consistently much stronger than the band in the input lane, indicating an efficient IP of all GFP labelled syntaxins. On the anti-HA blot, one clear band around $30 \mathrm{kDa}$ was visible for all input fractions corresponding to the 3 HA-VAMP fusion proteins. For all combinations, a band was also visible in the IP lane, representing the $3 \mathrm{HA}-\mathrm{VAMP}$ fusion proteins that co-purified with the GFP labelled SYPs. In general, the individual SNAREs show different co-IP levels: SYP121 and SYP131 interact stronger than both spliceforms of SYP132. VAMP724 interacts less than the other VAMPs. Most striking, in case of SYP132a the co-IPs of all VAMPs was extremely weak with signals barely detectable. To further confirm the ability of SYP132a to interact with VAMPs we used a BiFC approach in Nicotiana benthamiana leaves. This showed that SYP132a interacted with all tested VAMPs; VAMP721a, VAMP721d and VAMP721e (Supp. fig3). Thus, we consider it most likely that SYP132a does interact with all VAMPs, but that complexes are more rapidly disassembled.
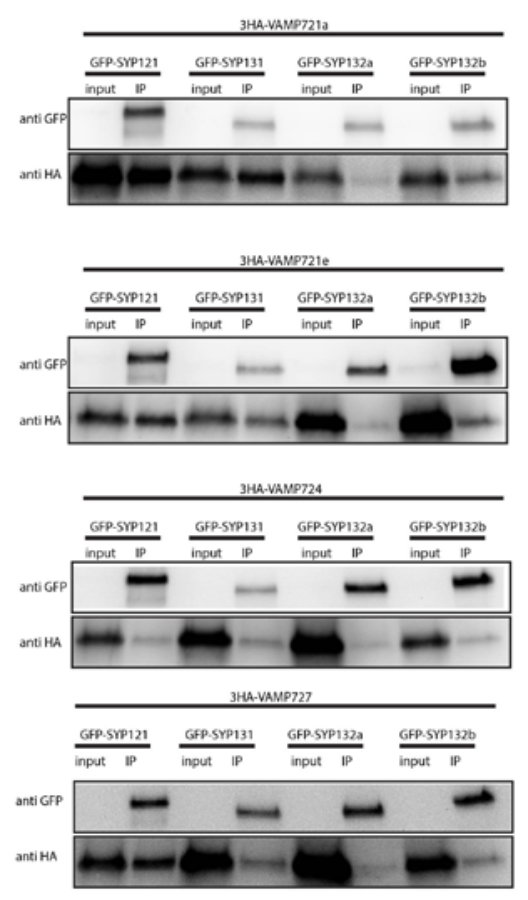

Figure 3. Co-immunoprecipitation analysis of SNARE interactions.

Representative western blot showing GFP-labelled syntaxins and 3HA-labelled VAMPs, expressed from the Medicago PT4 promoter, in extracts of mycorrhized Medicago roots before (input) and after immunoprecipitation (IP) using anti-GFP coated beads. 


\section{Symbiotic SNAREs are largely redundant with their non-symbiotic paralogs}

Since all of the analysed non-symbiotic syntaxins localize to the PAM, and are able to form SNARE complexes with any of the VAMPs, we wondered whether they are functionally redundant to the symbiotic SNAREs. To test this, we used a RNAi complementation approach. Therefore, we combined in one binary vector the RNAi constructs targeting SYP132a or VAMP721d/e (Ivanov et al., 2012, Chapter 3), and expression cassettes expressing the non-symbiotic SNAREs from the promoter of their symbiotic family member. As positive controls, we expressed VAMP721e lacking its native 3'UTR or codon-scrambled SYP132a, both of which escape silencing by the RNAi constructs. We used Agrobacterium rhizogenes mediated transformation to generate transgenic roots expressing these constructs. 6 weeks after inoculation with AM fungi, roots were harvested, and successful RNAi was confirmed in each individual root by qRT-PCR (Supp. fig4). Next, we quantified the mycorrhization level in the silenced roots as and determined the arbuscule phenotype by confocal microscopy after staining with WGA-Alexa488 (Fig 4b-g, i-m). Knockdown of SYP132a resulted in a severe reduction of mature arbuscules, as most arbuscules were stunted or collapsed. Expression of any of the tested syntaxins was sufficient to restore the arbuscule morphology of SYP132a knockdown to that of non-silenced control roots (Fig. 4b-g), although complementation with SYP121 resulted in a slightly lower amount of arbuscules that fill the whole cell (Fig. 4a). The phenotype of VAMP721d/e RNAi was slightly stronger than SYP132a RNAi. As described earlier, arbuscule formation does not proceed beyond the formation of the arbuscular trunk (Ivanov et al., 2012). Furthermore, the levels of mycorrhization were reduced (Fig. 4h). After expression of VAMP721a, VAMP721e or VAMP724, the arbuscule phenotype was fully restored (Fig. 4i-I), showing many mature arbuscules. In contrast, the arbuscule morphology was not restored after expression of VAMP727 (Fig. 4m). The levels of mature arbuscules after complementation with VAMP721a or VAMP724 were slightly lower than the empty vector control, but not significantly different from the positive control; complementation with VAMP721e itself (Fig. 4h).

Figure 4. RNAi complementation analysis.

A and $\mathrm{H}$; quantification of mycorrhized root length and arbuscule abundance according to Trouvelot et al., 1986 in Medicago roots transformed with different constructs. Error bars represent standard deviation of 3-4 biological replicates. Different letters indicate statistical difference $(\mathrm{p} \leq 0.05)$ determined using a pairwise Student's t-test. B-G and I-M; projection of confocal image stacks showing AM fungi in Medicago roots transformed with different constructs, stained with wheat germ agglutinin conjugated to alexa488. 

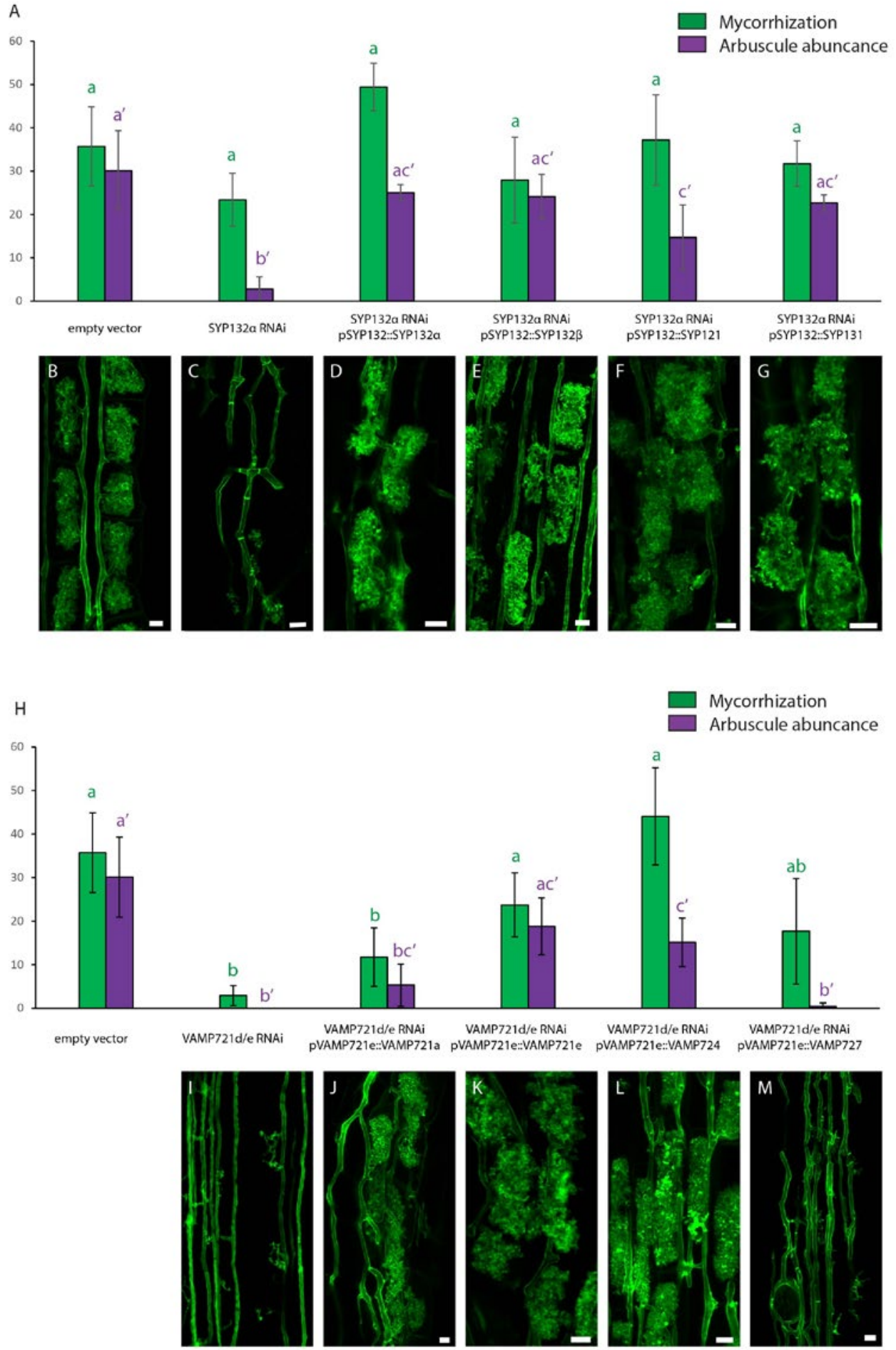


\section{Chapter 4}

\section{Table 1}

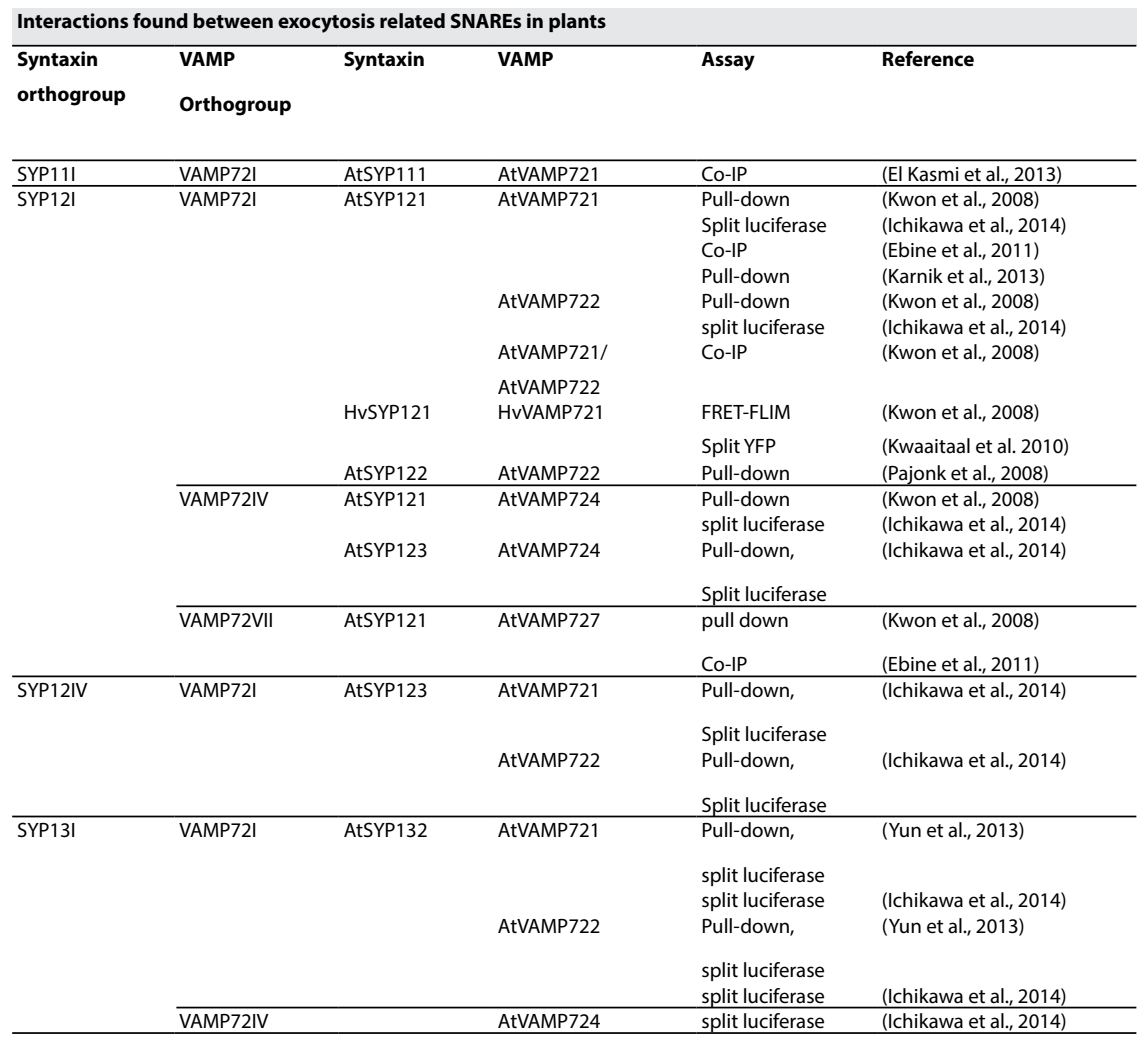

\begin{tabular}{|c|c|c|c|c|c|}
\hline $\begin{array}{l}\text { Syntaxin } \\
\text { orthogroup }\end{array}$ & $\begin{array}{l}\text { VAMP } \\
\text { Orthogroup }\end{array}$ & Syntaxin & VAMP & Assay & Reference \\
\hline SYP11I & VAMP72VII & AtSYP111 & AtVAMP727 & Co-IP & (Ebine et al., 2011) \\
\hline SYP12I & VAMP72I & AtSYP121 & $\begin{array}{l}\text { AtVAMP723 } \\
\text { (ER/pseudogene) }\end{array}$ & split luciferase & (Ichikawa et al., 2014) \\
\hline SYP12IV & VAMP72I & AtSYP123 & $\begin{array}{l}\text { AtVAMP723 } \\
\text { (ER/ pseudogene) }\end{array}$ & split luciferase & (Ichikawa et al., 2014) \\
\hline SYP13I & VAMP72I & AtSYP132 & $\begin{array}{l}\text { AtVAMP723 } \\
\text { (ER/ pseudogene) }\end{array}$ & $\begin{array}{l}\text { split luciferase } \\
\text { split luciferase }\end{array}$ & $\begin{array}{l}\text { (Yun et al., 2013) } \\
\text { (Ichikawa et al., 2014) }\end{array}$ \\
\hline
\end{tabular}

\section{DISCUSSION}

In this chapter, we have shown that the requirement for symbiotic SNAREs during the formation of arbuscules can largely be explained by their dominant expression levels in arbuscular cells. The lack of specificity in SNARE interactions and the ability of non-symbiotic SNAREs to complement for the loss of symbiotic SNAREs indicate that the symbiotic SNAREs do not mark a distinct secretory pathway. 
Based on the phylogeny of secretory SNAREs, several lines of evidence support the view that arbuscule formation does not depend on a dedicated trafficking pathway defined by SYP13II and VAMP72II: 1). Symbiotic SNAREs evolved after the evolution of AM symbiosis. Thus, the formation of an arbuscule did initially not depend on specialized SNAREs. 2). Symbiosis specialized VAMP72II evolved later than the symbiosis specialized SYP13II. Thus, the initial advantage of retaining SYP13/I (and still in monocots) could not be dependent on a selective interaction with VAMP72II. 3). VAMP72II is very similar to and embedded in the VAMP72I orthogroup. Furthermore, its conservation is not strictly following the ability of plants to host AM fungi. This can be explained by the functional overlap between VAMP72I and VAMP72II when expressed at sufficient level.

Does the diversification of exocytosis SNAREs in general allow multiple exocytosis pathways to co-exist in the same cell? In this study, we confirmed that the early separation of VAMP72VII from the other VAMPs in early seed plants did in fact facilitate a new trafficking pathway. MtVAMP727 mainly localizes to endosomes and the vacuole. This is in line with earlier studies on its Arabidopsis ortholog AtVAMP727, which accumulates on endosomes/pre-vacuolar compartments closely associated with the tonoplast (Uemura et al., 2004). AtVAMP727 is involved in vesicle fusion with the tonoplast by forming a SNARE complex with vacuolar Qa-SNARE AtSYP22, Qb-SNARE AtVTI11 and Qc-SNARE AtSYP51, and is required for the transport of protein storage vacuole marker CT24 to the vacuole (Ebine et al., 2008). Besides this, AtVAMP727 can also interact with AtSYP121 at the plasma membrane and is regulated by the plant specific RAB5-GTPase Ara6 to control endosome to plasma membrane traffic (Ebine et al., 2011). Thus, VAMP72VII defines a vesicle trafficking pathway that can be directed to the vacuole as well as to the plasma membrane (Ebine et al., 2011). After the appearance of VAMP72VII, VAMP72IV and VAMP72II evolved in angiosperms and dicots respectively. Here, we have shown that MtVAMP724 and MtVAMP721a can complement for the loss of MtVAMP721d/e. Thus, the minimal components for the formation of the PAM can also be recruited in MtVAMP724 and MtVAMP721a labelled vesicles, and are targeted to the PAM. Although it cannot be excluded that the cargo destined for the PAM is loaded into a different vesicle population in absence of VAMP721d/e coated vesicles.

Our co-IP analysis showed that there are no absolute specificity in the formation of SNARE complexes between the different secretory SNAREs, which is in line with and extends earlier studies on specificity of SNARE interactions in Arabidopsis (summarized in Table 1). Surprisingly though, very low amounts of VAMPs co-purified with the symbiotic SNARE SYP132a. The most straight-forward explanation would be that SYP132a does not interact with the tested VAMPs under these conditions. However, we consider this to be unlikely, since the possible SNARE partners of SYP132a are finite, and we tested members of all conserved VAMP orthogroups as well as all VAMPs that are expressed in arbuscule cells (with the exception of VAMP721d that is redundant and nearly identical to VAMP721e). 
The observation that RNAi of SYP132a can be complemented by both SYP121 and SYP131 shows that their role in vesicle fusion is conserved between these paralogs. Furthermore, using a BiFC approach we could confirm that SYP132a interacts with different VAMPs. These results raise the question what causes the observed difference in co-IP between different syntaxins. The actual event of vesicle fusion in which a trans-SNARE complex between the v-SNARE on the vesicle and a t-SNARE on the target membrane is formed, is followed by a phase were a cis-SNARE complex is present. In a cis-SNARE complex both $v$ - and t-SNAREs are present on the same membrane until they are disassembled by the ATPase NSF. It was recently shown that also before vesicle fusion SNAREs involved in cytokinesis are transported to their site of action as cis-SNARE complexes, which may apply to other secretory SNAREs as well (Karnahl et al., 2017). The actual vesicle fusion event is short-lived compared to the lifetime of cis-SNARE complexes. Therefore, it is likely that the complexes that are detected in our co-IP study - or any previous study on SNARE interactions in plants - are in fact mostly representing cis-SNARE complexes, which may be exaggerated by the strong expression from the PT4 promoter. In this scenario, the amount of VAMPs that co-purify with syntaxins is not a measure for the amount of vesicle fusion events driven by this particular complex, but merely represents the speed of cis-complex formation and dissociation. This implies that SYP132a containing SNARE complexes are more rapidly dissembled, possibly to recycle the syntaxin for subsequent fusion reactions at the PAM.

Since there does not seem to be specificity in SNARE interactions, and since both v- and t-SNARE homologs are highly redundant, the initial idea that distinct t-SNAREs control the selective fusion of vesicles labelled with different v-SNAREs is very unlikely. What is then the reason for the expansion of t-SNAREs in plants? Instead of marking different exocytosis pathways, it is more likely that plants limit vesicle fusion in general by controlling the expression and activity of t-SNAREs. In Arabidopsis, different secretory t-SNAREs are expressed in different plant tissues (Enami et al., 2009), show specific subcellular localization patterns (Lauber et al., 1997; Collins et al., 2003; Ângelo Silva et al., 2010; Ichikawa et al., 2014), turnover (Reichardt et al., 2011) or dynamics (Nielsen \& Thordal-Christensen, 2012). Besides the spatiotemporal presence of syntaxins, also their activity can be regulated on protein level. At their amino-terminal end, three helical domains ( $\mathrm{Ha}, \mathrm{Hb}$ and $\mathrm{Hc}$ ) are present that can fold back on the SNARE domain creating a so-called 'closed' conformation which prevents it from forming SNARE complexes. The regulation of the open and closed states by accessory proteins, such as Sec1/Munc18 proteins, allows additional control over vesicle fusion and prevents the formation of cis-SNARE complexes before vesicle fusion (Park et al., 2012; Grefen et al., 2015). Such accessory proteins have also been shown to control SNARE recycling after complex formation (Karnik et al., 2015). The relatively low amount of VAMPs that co-purified with SYP132a may therefore reflect a more stricter regulation of SYP132a activity compared to the other syntaxins. 
With the exception of VAMP727 that marks a different pathway, the symbiotic SNAREs are clearly the highest expressed exocytotic SNAREs in arbuscule cells. Previously, we have shown that different syntaxins are expressed in cells in which an arbuscule is formed (Huisman et al., 2016). This analysis was based on relative expression of genes measured by qPCR. The ddPCR approach used here is independent of primer efficiency and does not rely on pre-amplification of RNA or CDNA that could introduce artefacts, which makes it more suitable to compare the expression of different genes. Since the symbiotic SNAREs are the dominantly expressed secretory SNAREs, their evolutionary conservation can largely be explained by their expression pattern. The exception to this is the conservation of the two spliceforms of SYP13II. Since the alternative splicing of SYP13II does not affect the total level of SYP13/I transcripts, a difference in expression cannot explain the conservation of the two spliceforms. Therefore, they are likely functionally different. In line with this we observed a difference in localization at later stages of arbuscule development (Chapter 3). Furthermore, lower amount of VAMPs co-precipitated with SYP132a compared to SYP132 $\beta$, suggesting differential regulation of complex formation. The nature of the difference between both spliceforms may nevertheless be subtle since both are sufficient for arbuscule formation, although subtle effects on arbuscule lifetime or function may be undetectable by A. rhizogenes mediated root transformation.

The unique composition of the PAM does not appear to depend on a secretory pathway that is different from the secretory pathway directed to the plasma membrane. Instead, the difference between the plasma membrane and the PAM is largely the result of a drastic transcriptional change of cargo proteins during PAM formation, as shown by Pumplin and colleagues (Pumplin et al., 2012). Also in other plant cells where proteins are localizing to different plasma membrane domains, this does not seem to be the result of the use of different trafficking pathways, but the result of selective endocytosis and recycling of proteins (Geldner et al., 2003; Nielsen et al., 2012; Łangowski et al., 2016). Nevertheless, there are some indications that different secretory pathways co-exist. There is accumulating evidence that secretory vesicles can originate from different endomembrane compartments. Instead of the conventional route from ER via Golgi and trans-Golgi network (TGN) to the plasma membrane, secretion of pectin methylesterase in tobacco pollen tubes bypasses the TGN (Wang et al., 2016). Furthermore, recycling of the auxin efflux carrier PIN1 is shown to be insensitive to the chemical inhibitor of post-Golgi traffic Endosin 16, suggesting that it bypasses the TGN as well (Li et al., 2017). Finally, the recycling of different auxin efflux carriers in the epidermis is differentially sensitive to ARF-GFF inhibitor Brefeldin A (Kleine-Vehn et al., 2008). Together, these results show that not all cargo destined for the plasma membrane follows the same route, suggesting the existence of different secretory pathways. Based on our data, we consider a regulatory role for SNAREs in separating these pathways unlikely. How such different secretory pathways are regulated instead remains to be revealed. 


\section{MATERIALS AND METHODS}

\section{Phylogenetic analysis of SNAREs}

The protein sequences of all SYP1 and VAMP72 family members were retrieved from the Phytozome database (www.phytozome.net) for the AM host species; Aquilegia coerulea, Brachipodium distachyon, Citrus clementina, Carica papaya, Cucumis sativus, Fragaria vesca, Ginkgo biloba, Musa acuminate, Manihot esculenta, Medicago truncatula, Oryza sativa, Phoenix dactylifera, Prunus persica, Populus trichocarpa, Setaria italic, Solanum lycopersicum, Selagionella moelendorfii, Theobroma cacao and Vitis vinifera, and the AM non-host species; Arabidopsis thaliana, Beta vulgaris, Dianthus caryophyllus, Marchantia polymorpha, Nelumbo nucifera, Physcomitrella patens, Pinus taeda, Striga hermonthica, Spirodela polyrhiza and Utricularia gibba. To ensure the recovery of all family members, missing species in each orthogroup were confirmed by repeated homology searches using orthologs from that group as an input. The species were choses to include a large and diverse range of AM hosts and non-hosts. The Protein sequences were aligned in Mega5 using the ClustalW algorithm. Subsequently, trees were constructed using the neighbour-joining method with 100 bootstrap iterations.

\section{Plant growth and mycorrhization}

Medicago truncatula Jemalong A17 was used in all experiments. For transformation, Agrobacterium rhizogenes MSU440 was used according to (Limpens et al., 2004). For mycorrhization assays, plants were transferred to pots containing a 5:3 $(\mathrm{v} / \mathrm{v})$ ratio mix of expanded clay and sand, saturated with modified Hoagland medium $\left(5 \mathrm{mM} \mathrm{MgSO}_{4^{\prime}} 2.5\right.$ $\mathrm{mM} \mathrm{Ca}\left(\mathrm{NO}_{3}\right)_{2^{\prime}} 2.5 \mathrm{mM} \mathrm{KNO}_{3^{\prime}}, 2 \mathrm{mM} \mathrm{NH}_{4} \mathrm{NO}_{3^{\prime}} 500 \mu \mathrm{M}$ MES, $50 \mu \mathrm{M} \mathrm{NaFeEDTA}, 20 \mu \mathrm{M} \mathrm{KH}_{2} \mathrm{PO}_{4^{\prime}}$ $12,5 \mu \mathrm{M} \mathrm{HCl}, 10 \mu \mathrm{M} \mathrm{H}_{3} \mathrm{BO}_{3^{\prime}} 2 \mu \mathrm{M} \mathrm{MnCl}_{2^{\prime}} 1 \mu \mathrm{M} \mathrm{ZnSO}_{4^{\prime}} 0.5 \mu \mathrm{M} \mathrm{CuSO}_{4^{\prime}} 0.2 \mu \mathrm{M} \mathrm{Na}_{2} \mathrm{MoO}_{4^{\prime}} 0.2$ $\mu \mathrm{M} \mathrm{CoCl}{ }_{2}, \mathrm{pH}$ 6.1). Plants were inoculated with dried Rhizophagus irregularis infected maize roots obtained from Plant Health Cure (http://www.phc.eu). Plants were grown for four weeks at $21^{\circ} \mathrm{C}$ at a $16 / 8 \mathrm{~h}$ light/dark regime.

\section{Laser capture microdissection and ddPCR}

Roots of mycorrhized Medicago plants and uninfected control plants were harvested and fixed in Farmer's fixative (75\% ethanol, 25\% acetic acid) substituted with 0,01\% Chlorazol Black E to stain AM fungi, and vacuum infiltrated for 30 minutes on ice. Then, the roots were incubated in Farmer's fixative for 16 hours at $4^{\circ} \mathrm{C}$ on a spinning wheel. After fixation, the roots were dehydrated in an ethanol dehydration series $(80 \%, 85 \%, 90 \%, 95 \% 30$ minutes each followed by $100 \%$, overnight) Steedman wax was prepared by mixing $90 \%$ polyethylene glucol400 distearate and $10 \%$ 1-hexadecanol at $65^{\circ} \mathrm{C}$. Steedman wax was infiltrated by incubating the roots in $50 \%$ Steedman wax and $50 \%$ ethanol for two hours at $38^{\circ} \mathrm{C}$, followed by 3 incubations in $100 \%$ Steedman wax for 2 hours at $38^{\circ} \mathrm{C}$. Finally, 
the samples were transferred to room temperature to allow the wax to solidify. Solidified blocks of Steedman wax were cut into $20 \mu \mathrm{m}$ thick sections using a microtome, and transferred to PEN-membrane slides (Leica). Arbuscule containing cells and uninfected cortical cells were harvested using a Leica LMD7000 laser capture microdissection microscope. RNA was isolated using a RNeasy micro kit (Qiagen). cDNA was synthesized using the iScript cDNA synthesis kit (Bio-Rad). in a total volume of $20 \mu \mathrm{l} .1 \mu \mathrm{l}$ cDNA was then used per ddPCR reaction. For this, a ddPCR mastermix containing evaGreen as a probe was used (BioRad), as well gene specific primers (1-24, Table S1). Then the PCR mix was suspended in oil using the QX200 Droplet Generator (Biorad). The PCR was carried out following manufacturer's instructions. Subsequently, the absolute number of positive droplets was counted using a QX200 Droplet Reader.

\section{RNA isolation, CDNA synthesis, qRT-PCR}

RNA was isolated from plant tissue using the EZNA Plant RNA mini kit (omega). cDNA was synthesized from $1 \mu \mathrm{g}$ of RNA using the iScript cDNA synthesis kit (BioRad). Equal amounts of cDNA were used for qPCR using SYBR green supermix (Bio-Rad) in a Bio-Rad CFX connect real-time system qPCR machine. Gene expression levels were determined using gene specific primers listed in table S1. The gene expression was normalized using Actin2 and Ubiquitin 10 as reference genes.

\section{Plasmid construction}

The SYP132a RNAi construct and the empty RNAi control vector are described in chapter 3. The VAMP721d/e RNAi vector was described in (Ivanov et al., 2012). All other expression cassettes were created using multisite Gateway technology (Invitrogen) in the pKGWRR-MGW destination vector (Ovchinnikova et al., 2011). For all reactions a pENTR2-3 carrying a 35s terminator was used. For GFP fusions driven by the PT4 promoter, the pENTR4-1 described in chapter 3 was used. To obtain a pENTR4-1 containing a triple HA-tag driven by the PT4 promoter, A triple HA tag flanked by Ascl and Acc65I restriction sites was de-novo synthesized (Integrated DNA technologies). Using Ascl-Acc65I restriction-ligation, The GFP in the pENTR4-1 pPT4::GFP was swapped for the triple HA-tag. pENTR2-3 vectors containing nGFP or cGFP were obtained from VIB Ghent. For n-terminal split GFP fusions, nGFP and CGFP were amplified from these vectors using primers 37-40 listed in table S1. Primers were designed to remove stop codons, and to add a start codon and Ascl/Acc65I restriction sites. Subsequently, both fragments were cloned into a pENTR4-1 vector containing a $35 \mathrm{~S}$ promoter using Ascl-Acc65I restriction/ligation. The pENTR1-2 vectors containing the coding sequences of SYP121, SYP122, SYP132a and SYP132 $\beta$ and the pENTR4-1 carrying the SYP132 promoter are described in chapter 3 . The pENTR1-2 vectors containing the coding sequences of VAMP721a, VAMP721d and VAMP721e, and the pENTR4-1 carrying the VAMP721e promoter were described in (Ivanov et al., 2012). 
The pENTR1-2 vectors containing the coding sequence of SYP131, VAMP724 and VAMP727 were constructed by amplifying the respective genes from Medicago A17 cDNA using primers 25-30, adding a cacc sequence adapter at the 5' end. The PCR fragment was then cloned into a pENTR/D-TOPO entry vector using TOPO cloning (Invitrogen). To combine GFP fusion cassettes with 3HA fusion cassettes, the GFP fusion constructs were amplified using primers 31 and 32 (Table S1), adding Spel and Swal restriction sites. The HA fusion constructs were amplified using primers 33 and 34 (Table S1), adding Swal and Apal restriction sites. Using 3-point restriction/ligation, the two constructs were inserted into a pKGW-MGW binary vector. To combine SNARE expression cassettes with RNAi constructs for RNAi complementation, the SNARE expression cassette was apmplified using primers 35 and 36 (Table S1), adding Apal and Eco81I restriction sites. Using Apal-Eco81I restriction/ligation, the cassettes were inserted into the SYP132 $a$ and VAMP721d/e RNAi vectors.

\section{Microscopy}

For confocal imaging, a Leica SP8 confocal microscope was used. An excitation wavelength of $488 \mathrm{~nm}$ was used for WGA-alexa488 and GFP. An excitation wavelength of 543 $\mathrm{nm}$ was used for dsRed. Appropriate emission filter settings were used to separate the fluorophores used in each experiment.

\section{Co-Immunoprecipitation}

The roots of mycorrhized plants (5 weeks post inoculation) expressing different combinations of GFP-labelled syntaxins and 3HA-labeled VAMPs were harvested, and residual sand was washed away. The roots were flash-frozen in liquid nitrogen, and ground using a mortar and pestle. One gram of plant material was added to $6 \mathrm{ml}$ of RIPA buffer $(10 \mathrm{mM}$ Tris/Cl pH 7,5, 150mM NaCl, 0,1\% SDS, 1\% triton X-100, 1\% Sodium Deoxycholate, 0,5mM

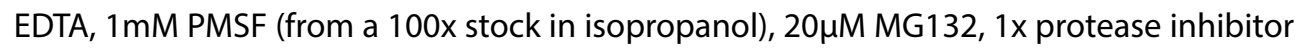
mix (Roche cOmplete, EDTA free)), and ground in a potter tube. After 20 minutes incubation on ice, the extracts were centrifuged twice at $9000 \mathrm{~g}$ after which the supernatant was transferred to a new tube each time. The input fraction was harvested at this point, and mixed in a 1:1 ratio with 4 X SDS-sample buffer (200mM TrisHcl 6.8, 8\% SDS, 40\% glycerol, $4 \% \beta$-mercaptoethanol, 50mM EDTA, $0.08 \%$ bromophenol blue). Then $30 \mu \mathrm{l}$ of anti-GFP coated agarose beads (Chromotek), equilibrated in washing buffer (50mM TrisHcl8.0, $150 \mathrm{mM} \mathrm{NaCl}, 0.1 \%$ Triton X-100, 1x protease inhibitor mix (Roche cOmplete, EDTA free)) were added to the protein extracts. The samples were incubated for 1 hour at $4{ }^{\circ} \mathrm{C}$ on a spinning wheel. Then, the beads were harvested by centrifugation at $2000 \mathrm{~g}$ for $2 \mathrm{~min}$, and washed 3 times with $1 \mathrm{ml}$ of washing buffer. The washing buffer was removed, and $100 \mu \mathrm{l}$ 2x SDS-sample buffer was added, after which the samples were incubated for $10 \mathrm{~min}$ at 98 ${ }^{\circ} \mathrm{C}$. After centrifugation for $2 \mathrm{~min}$ at $2700 \mathrm{~g}$, the supernatant was transferred to a new tube and stored at $-20^{\circ} \mathrm{C}$ until gel electrophoresis. 


\section{Western blotting}

Proteins were separated on a precast $4-12 \%$ poly acrylamide gradient mini-gel (Bio-Rad) at $300 \mathrm{~V}$. Then the proteins were transferred to a PVDF membrane using the BioRad TransBlot turbo system. The blot was blocked for 1 hour with 3\% BSA in TBST (50mM TrisHcl 7.4, $150 \mathrm{mM} \mathrm{NaCl}, 0.3 \%$ Tween 20) while shaking and washed 3 times with TBST. Antibodies against GFP (Miltenyi Biotec) or HA (Pierce scientific) conjugated to horse radish peroxidase diluted 5000x in TBST with 1\% BSA were added, and incubated for 1 hour while shaking. Then, the blot was washed 3 times with TBST, and one time with TBS $(50 \mathrm{mM}$ TrisHcl 7.4, $150 \mathrm{mM} \mathrm{NaCl}$ ). Finally, $1 \mathrm{ml}$ of supersignal west femto ECL substrate (Thermo scientific) was added to the blot, and luminescence was measured for 5 minutes, using a G:box detection system (Syngene).

\section{Agrobacterium infiltration of Nicotiana benthamiana}

Agrobacterium tumefaciens C58 expressing split-GFP constructs were grown in liquid LB with appropriate antibiotics for 2 days at $28^{\circ} \mathrm{C}$. The bacteria were collected by centrifugation, resuspended in MMi medium (10 g/l sucrose, $5 \mathrm{~g} / \mathrm{l}$ MS basal salts (Duchefa), $2 \mathrm{~g} / \mathrm{l}$ MES, $200 \mu \mathrm{M}$ acetosyringone, $\mathrm{pH}$ 5.6) to an OD600 of 0.1 and incubated for 1 hour at room temperature. Different combinations of split-GFP constructs were made by mixing the appropriate bacterial suspensions in a 1:1 ratio. The suspensions were then injected into the leaves of Nicotiana benthamiana plants which were then grown in a greenhouse at 21 ${ }^{\circ} \mathrm{C}$. Three days post infiltration, the infiltrated parts were analysed by confocal microscopy.

\section{AM staining and quantification}

For quantification of mycorrhization, roots were incubated in $10 \%(\mathrm{w} / \mathrm{v}) \mathrm{KOH}$ at $98{ }^{\circ} \mathrm{C}$ for 10 minutes. Then roots were washed 3 times with $5 \%$ acetic acid. After washing, the roots were stained in $5 \%$ ink in $5 \%$ acetic acid, for 2 minutes at $98{ }^{\circ} \mathrm{C}$. after staining the roots were destained in $5 \%$ acetic acid, refreshing the destaining solution several times. For staining with WGA alexafluor 488 , roots were incubated in $10 \%(\mathrm{w} / \mathrm{v}) \mathrm{KOH}$ at $60{ }^{\circ} \mathrm{C}$ for 3 hours. Then, roots were washed 3 times in PBS $\left(150 \mathrm{mM} \mathrm{NaCl}, 10 \mathrm{mM} \mathrm{Na} \mathrm{HPO}_{4^{\prime}} 1.8 \mathrm{mM}\right.$ $\mathrm{KH}_{2} \mathrm{PO}_{4^{\prime}} \mathrm{pH} 7.4$ ), and incubated in $0.2 \mu \mathrm{g} / \mathrm{mL}$ WGA-Alexafluor 488 (Molecular Probes) in PBS at room temperature for 16 hours. For quantification of mycorrhization, roots were cut into $1 \mathrm{~cm}$ fragments, and the colonization and arbuscule abundance was scored and calculated according to Trouvelot et al.(Trouvelot et al., 1986).

\section{ACKNOWLEDGEMENTS}

We thank Ilias Gkikas and Kate Ligthart for their contribution to this project 


\section{SUPPLEMENTAL INFORMATION}

Table S1. Primers used in this study.

\begin{tabular}{|c|c|c|c|}
\hline \multirow{25}{*}{ ddPRC } & number & name & Sequence $5^{\prime} \rightarrow 3^{\prime}$ \\
\hline & 1 & SYP111F & TCCCATTGTCACTAGTTTCAGC \\
\hline & 2 & SYP111R & GGCAATTCAAACGACGATG \\
\hline & 3 & SYP121F & ATGCAGCAGCTATTCCCATC \\
\hline & 4 & SYP121R & TTCACAAGGTTTGCCTCACC \\
\hline & 5 & SYP122F & AGCGAGGAAGAACAAGATCAG \\
\hline & 6 & SYP122R & CCGATTGTAACCCTTGTTCC \\
\hline & 7 & SYP123F & TCTCAAATGGTGGCTACTGG \\
\hline & 8 & SYP123R & TGATGCTTGACTTGACTACCAAG \\
\hline & 9 & SYP131F & CGGTAACACATTGTITCCACAAC \\
\hline & 10 & SYP131R & GAGACCAATGTTTGTTTCAACC \\
\hline & 11 & SYP132AF & CAGCAGTTTAACAAGGTTCATGG \\
\hline & 12 & SYP132AR & AATATGCACAGAAAGCCAATTC \\
\hline & 13 & SYP132BF & GTTGTGTTGGTTGGGAAAGC \\
\hline & 14 & SYP132BR & CAGAGACACACCGATGTATTAGC \\
\hline & 15 & V721AF & AGGAAGGGAAGTGAATAAGGTGTG \\
\hline & 16 & V721AR & AGCATGCTTTCTATACACAATTCC \\
\hline & 17 & V721DF & TTGTAAATTGTTTGCTTTGCG \\
\hline & 18 & V721DR & AAAAAGCCACAGGTCCAATC \\
\hline & 19 & V721EF & AAGCTITTACAGTATGATGGTGATG \\
\hline & 20 & V721ER & ATGATAACAGGATGGGTCGG \\
\hline & 21 & V724F & AAGAAAATCAACCCAGGAGGG \\
\hline & 22 & V724R & CTITATCCACCAGCAACAAGG \\
\hline & 23 & V727F & TTGTACATTGAATTTGGTGCG \\
\hline & 24 & V727R & CTTGATTCAAAGTGACCAGCAG \\
\hline \multirow{2}{*}{$\begin{array}{l}\text { Cloning cds } \\
\text { SYP131 }\end{array}$} & 25 & cdsSYP131F & CACCATGAATGATCTTCTAACGGAATCA \\
\hline & 26 & cdsSYP131R & CTATGCACCCTITITGGTAACC \\
\hline \multirow{2}{*}{$\begin{array}{l}\text { Cloning cds } \\
\text { VAMP724 }\end{array}$} & 27 & cdsVAMP724F & CACCATGAGTCAAGAATCGTTCATATACAGC \\
\hline & 28 & cdsVAMP724R & CTAATTTGAACAGTTAAATCCACCG \\
\hline \multirow{2}{*}{$\begin{array}{l}\text { Cloning cds } \\
\text { VAMP727 }\end{array}$} & 29 & cdsVAMP727F & CACCATGAGTCAAAGGGGTTTGATATAT \\
\hline & 30 & cdsVAMP727R & TCAACATTTGAAACCCCCAC \\
\hline \multirow{6}{*}{$\begin{array}{l}\text { PCR expression } \\
\text { cassettes }\end{array}$} & 31 & MGWSpeI_F & TGACTAGTACGCCAAGCTATCAACTTTGT \\
\hline & 32 & MGWSwaI_R & GCATTTAAATCACGACGGCCAGTGAAT \\
\hline & 33 & MGWSwaI_F & TGATTTAAATACGCCAAGCTATCAACTTTGT \\
\hline & 34 & MGWApaI_R & TCATAACGTGACTCCСТTAATTCTC \\
\hline & 35 & MGWApaI_F & TAGGGCCCTACGCCAAGCTATCAACTTTGT \\
\hline & 36 & MGWEco81I_R & AACCTTAGGTCACGACGGCCAGTGAAT \\
\hline \multirow{4}{*}{$\begin{array}{l}\text { Split-GFP vector } \\
\text { construction }\end{array}$} & 37 & AscI-GFPnF & AGGCGCGCCATGGTGAGCAAGGGCGAG \\
\hline & 38 & Acc65I-GFPnR & AGGTACCGGCCATGATATAGACGTTGTG \\
\hline & 39 & AscI-START-GFPcF & AGGCGCGCCATGGACAAGCAGAAGAACGGCAT \\
\hline & 40 & Acc65I-GFPcR & AGGTACCCTTGTACAGCTCGTCCATGC \\
\hline
\end{tabular}




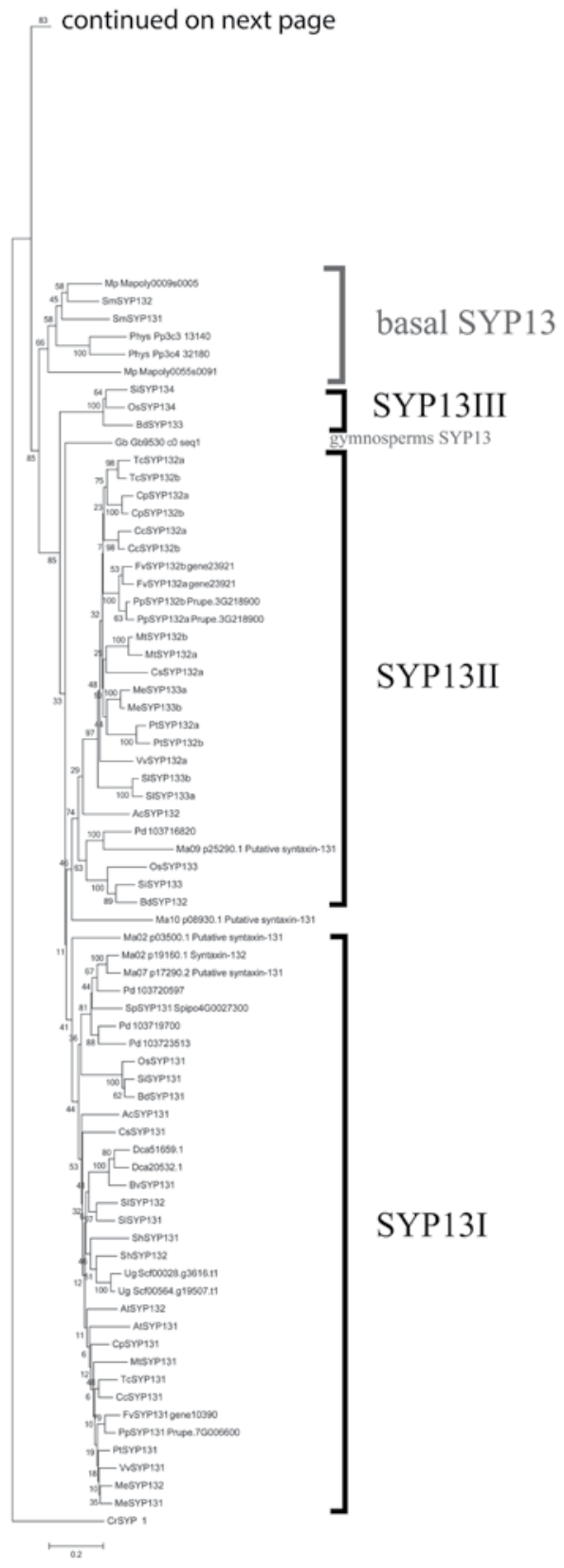

Supplemental figure 1. Neighbour-joining tree showing the SYP1 protein family. Included AM-host (black) and non-host (red) species are: Aquilegia coerulea (Ac), Arabidopsis thaliana (At), Brachipodium distachyon (Bd), Beta vulgaris (Bv), Citrus clementina (CC), Chlamydomonas reinhardtii (Cr), Carica papaya (Cp), Cucumis sativus (Cs), Dianthus caryophyllus (Dc), Fragaria vesca (Fv), Ginkgo biloba (Gb), Musa acuminate (Ma), Manihot esculenta (Me), Marchantia polymorpha (Mp), Medicago truncatula (Mt), Nelumbo nucifera (Nn), Oryza sativa (Os), Phoenix dactylifera (Pd), Physcomitrella patens (Phys), Pinus taeda, Prunus persica (Pp) Populus trichocarpa (Pt), Striga hermonthica (Sh), Setaria italic (Si) Solanum lycopersicum (SI), Selagionella moelendorfii (Sm), Spirodela polyrhiza (Sp), Theobroma cacao (Tc), Utricularia gibba (Ug), Vitis vinifera (Vv). 


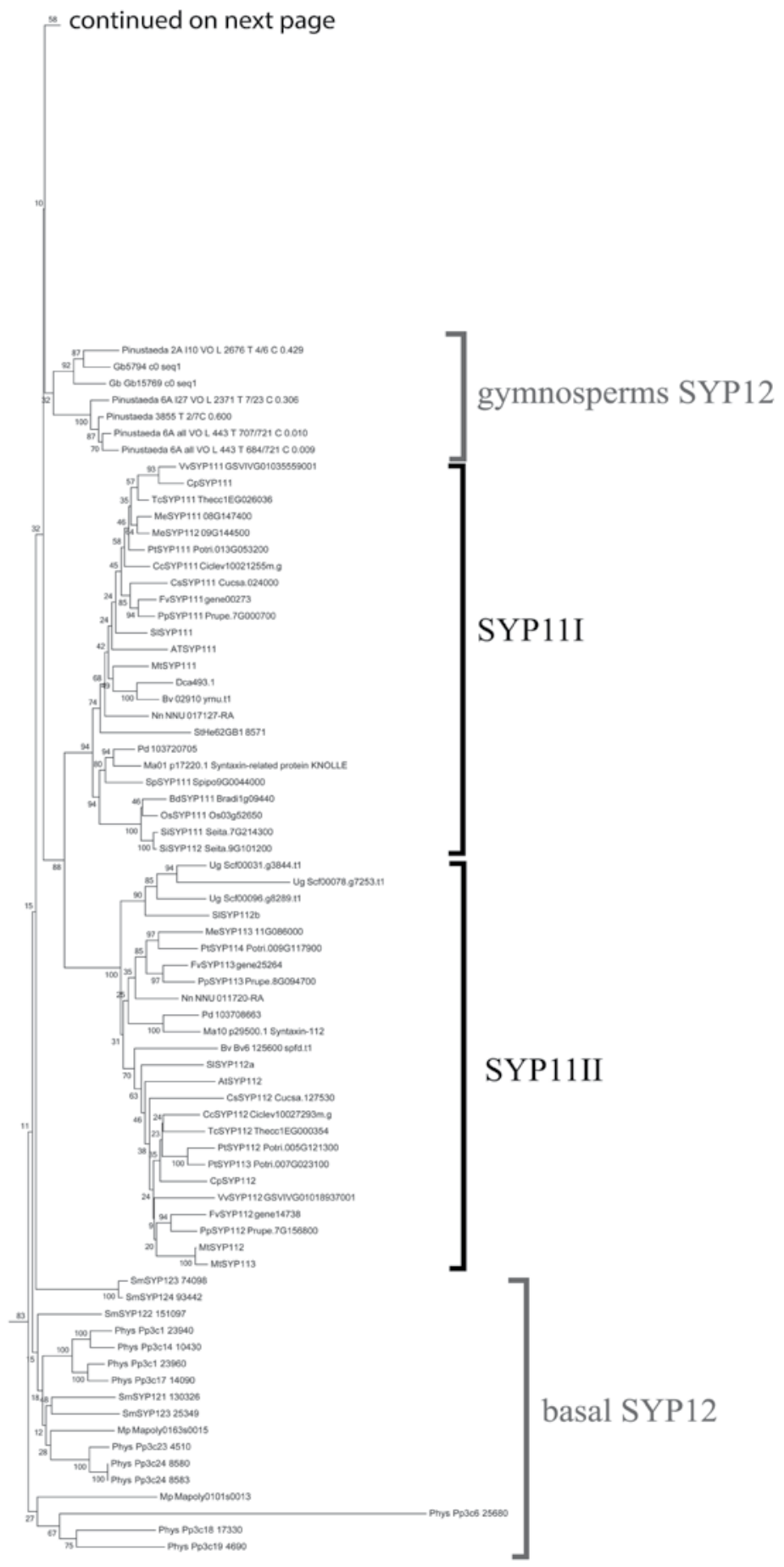




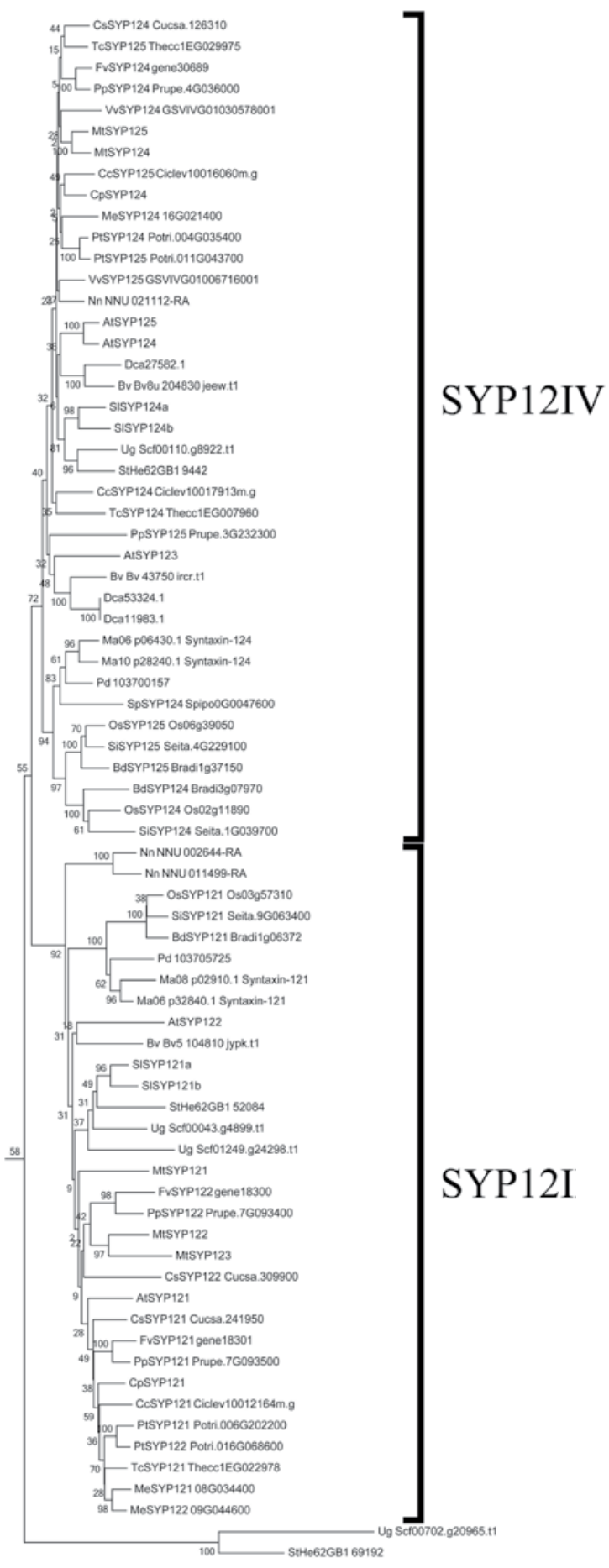




\section{Chapter 4}

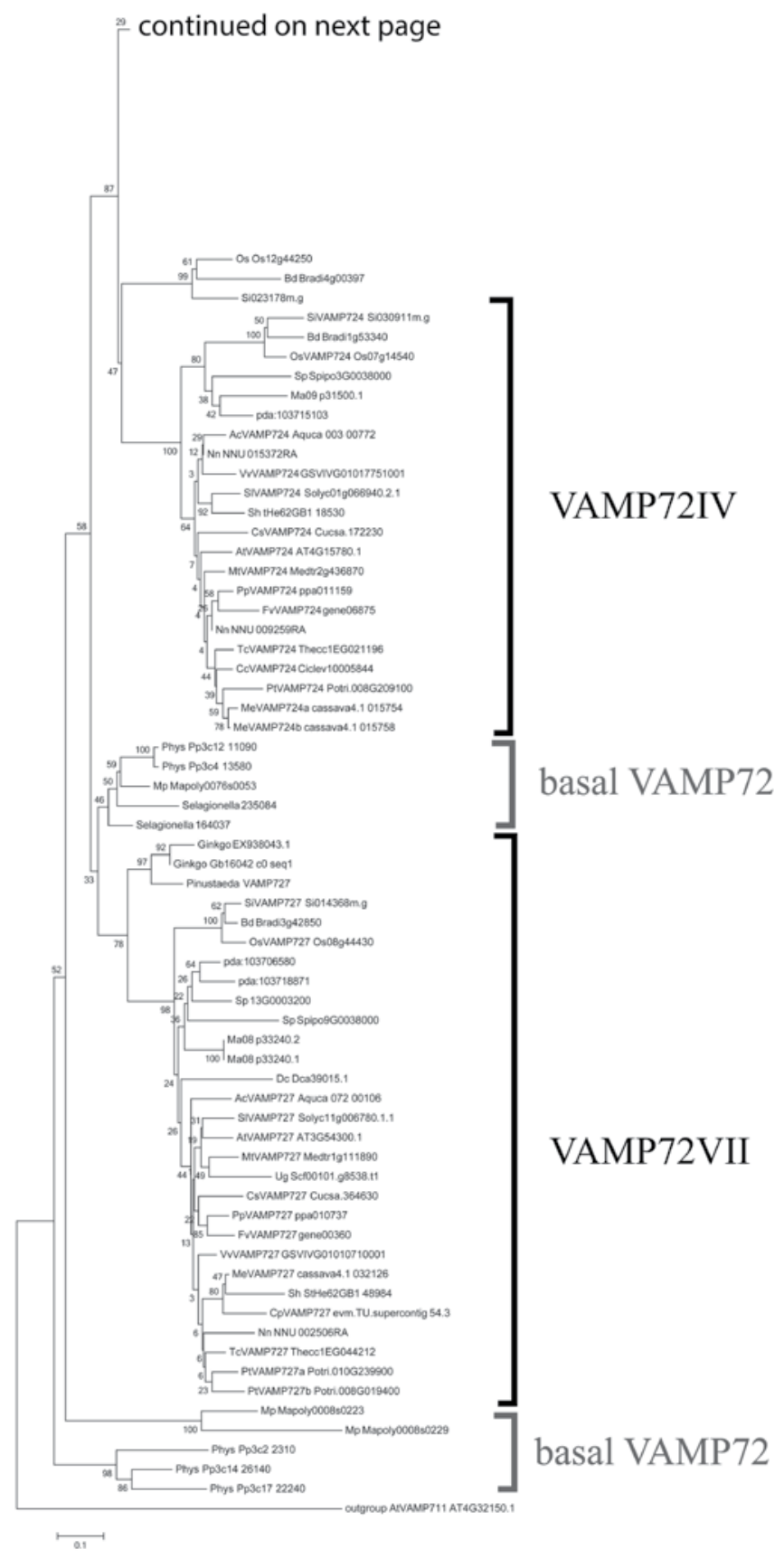

Supplemental Figure 2. Neighbour-joining tree showing the VAMP72 family. Included AM-host (black) and non-host (red) species are: Aquilegia coerulea (Ac), Arabidopsis thaliana (At), Brachipodium distachyon (Bd), Beta vulgaris (Bv), Citrus clementina (Cc), Carica papaya (Cp), Cucumis sativus (Cs), Dianthus caryophyllus (DC), Fragaria vesca (Fv), Ginkgo biloba (Gb), Musa acuminate (Ma), Manihot esculenta (Me), Marchantia polymorpha (Mp), Medicago truncatula (Mt), Nelumbo nucifera (Nn), Oryza sativa (Os), Phoenix dactylifera (Pda), Physcomitrella patens (Phys), Pinus taeda, Prunus persica (Pp) Populus trichocarpa (Pt), Striga hermonthica (Sh), Setaria italic (Si) Solanum lycopersicum (SI), Selagionella moelendorfii, Spirodela polyrhiza (Sp), Theobroma cacao (Tc), Utricularia gibba (Ug), Vitis vinifera $(V v)$. 


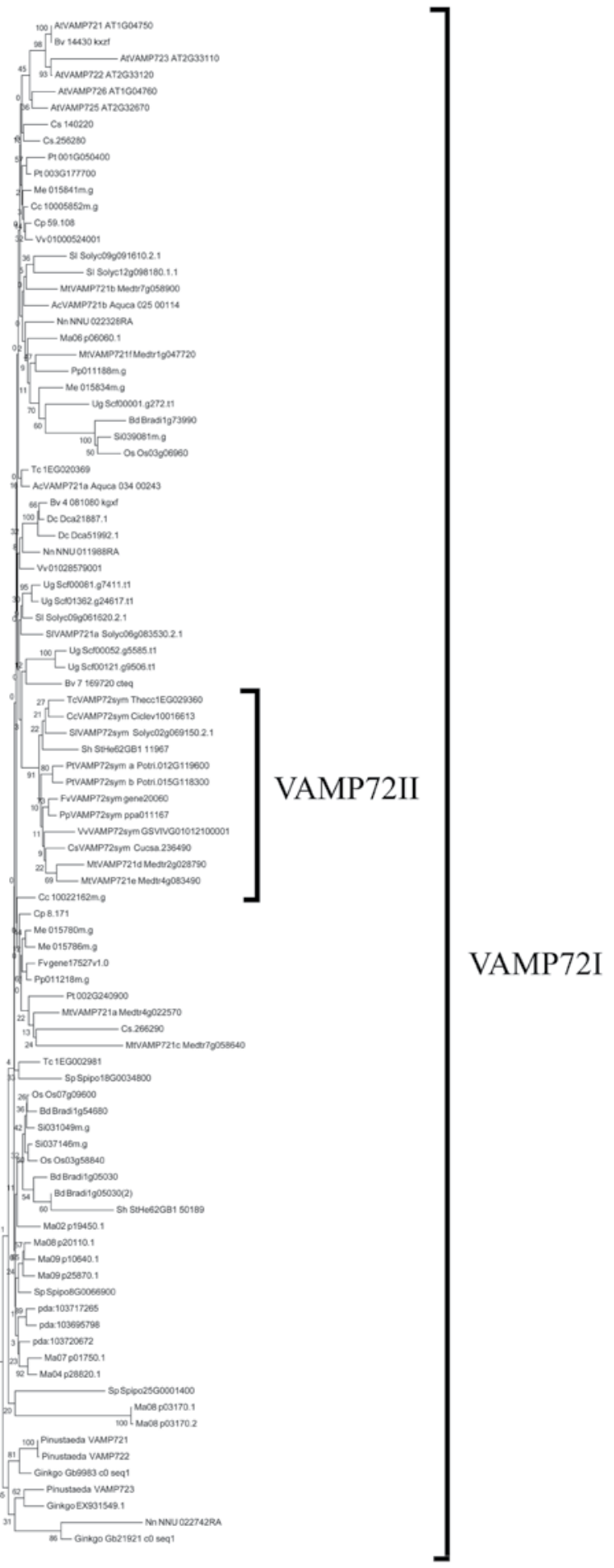




\section{Chapter 4}

GFPC-VAMP721a

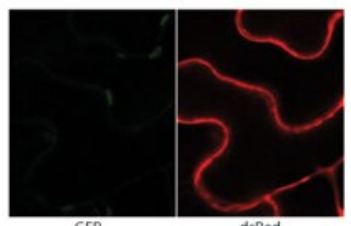

GFP

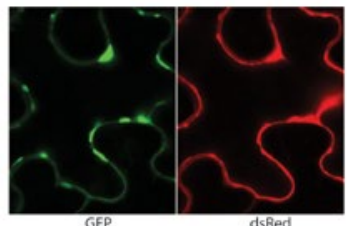

GFP
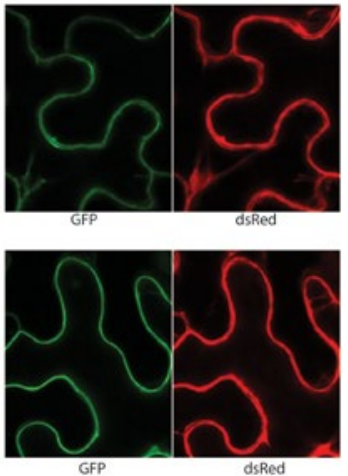

dsRed

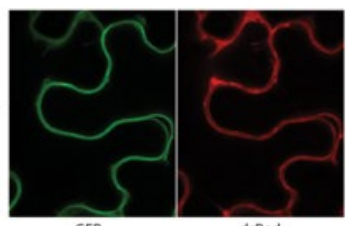

GFPc-VAMP721d

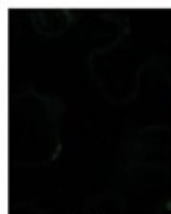

GFP
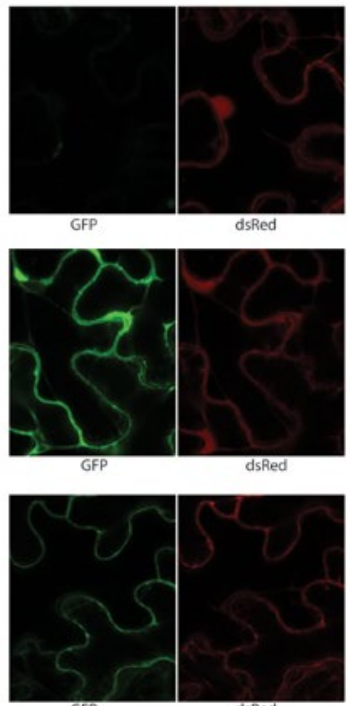

GFP

dsRed
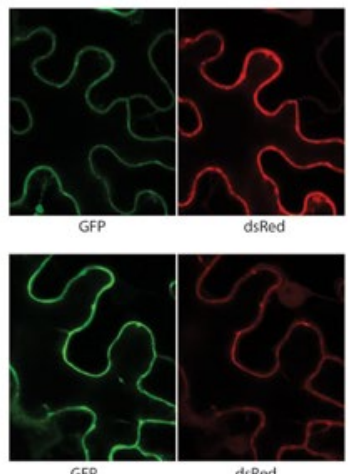

GFPc-VAMP721e

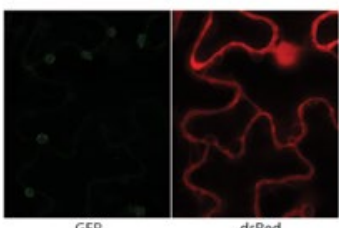

GFP

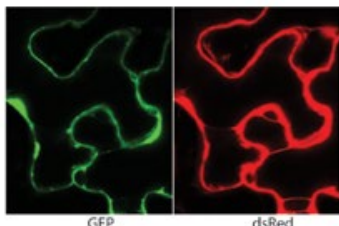

GFP
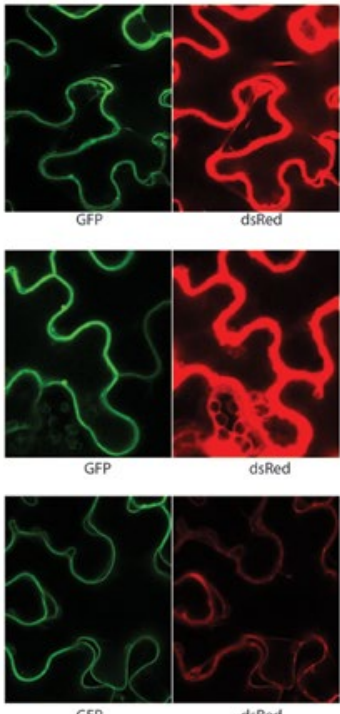

GFP

dsRed

\section{Supplemental figure 3. Split GFP analysis of SNARE interactions.}

Confocal images of Nicotiana benthamiana leaves transiently expressing different combinations of split-GFP fusion constructs. Syntaxins SYP121, SYP122, SYP132 $\beta$ and SYP132a, and plasma membrane protein MtPIP2.7 (negative control) fused to the n-terminal half of GFP are co-expressed with symbiotic v-SNAREs VAMP721d, VAMP721e, and their non-symbiotic paralog MtVAMP721a fused to the c-terminal half of GFP. Combinations of all syntaxins, but not MtPIP2.7, with all VAMPs results in a green fluorescent signal on the plasma membrane. 

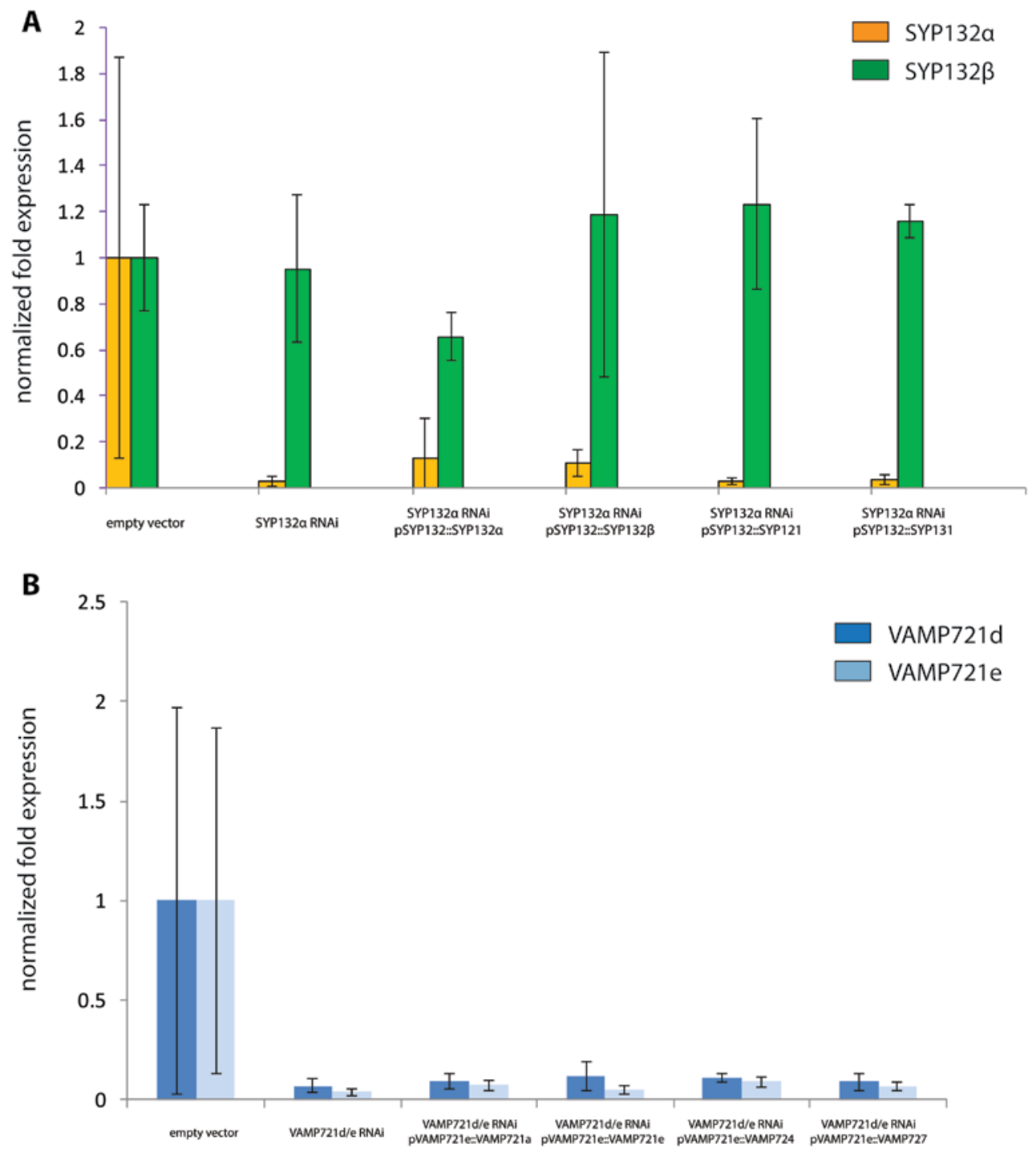

\section{Supplemental figure 4.}

qRT-PCR showing the expression of SYP132 and VAMP721d/e in RNAi complementation experiments shown in figure 4. Error bars represent standard deviation of 3-4 biological replicates. 
o

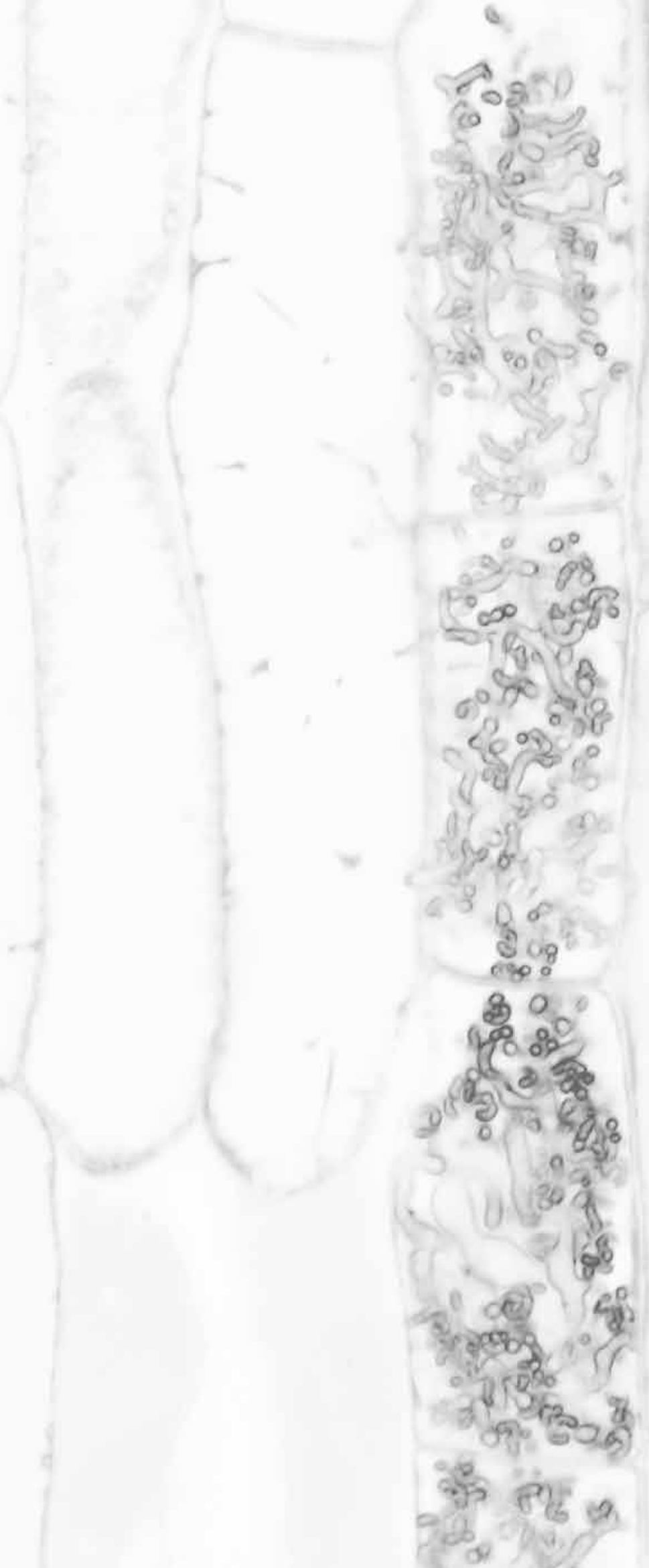




\section{Chapter 5}

\section{Specialization of SYP132a to control arbuscule functionality}

\section{Abstract}

Rik Huisman, Jan Hontelez, Ton Bisseling and Erik Limpens

Arbuscular mycorrhizal fungi and nitrogen fixing rhizobia are hosted inside living plant cells where they are surrounded by a plant derived membrane that forms a specialized host-microbe interface. We have previously shown that the formation of a stable hostmicrobe interface depends on the t-SNARE SYP132a, a symbiosis dedicated spliceform of SYP132. The dependence of host-microbe interface formation on SYP132 can be largely explained by its dominant expression in cells that host microbes. However, the conservation of SYP132 alternative splicing in the dicot lineage suggests that the two spliceforms do functionally differ, as splicing does not necessarily affect the total level of SYP132 transcripts. Here, we generated syp132a-1, a mutant line in which SYP132 is constitutively spliced into the SYP132 $\beta$ isoform, which allowed us to study the difference between SYP132 $\alpha$ and $-\beta$ in more detail. We show that arbuscules are formed in this line that, in number, morphology and lifetime, are indistinguishable from arbuscules in wild type plants. Nevertheless, the biomass of mycorrhized syp 132a-1 mutants remains smaller when these plants are mycorrhized, suggesting that the arbuscules are not fully functional. By using fluorescent timer fusions to track the turnover of proteins, we found that SYP132a localizes preferentially to functional arbuscule branches, as a result of increased stability or recycling of SYP132a compared to its non-symbiotic isoform.Our results show that SYP132 $\alpha$ is functionally different from SYP132 $\beta$, and required for the full functionality of arbuscules. 


\section{INTRODUCTION}

Arbuscular mycorrhizal (AM) fungi and rhizobium bacteria are hosted inside living plant cells during endosymbiosis with plants. This requires the formation of specialized host-derived membranes that surround the microbes. These membranes form an intimate host-microbe interface that facilitates optimal nutrient exchange between plant and microbe. We have shown that the formation of the host-microbe interface depends on a specific set of SNARE proteins. In Medicago two V-SNAREs (MtVAMP721- $d$ and $-e$ ) as well as an alternative splice-isoform of the t-SNARE SYNTAXIN132 (MtSYP132a) were shown to be essential for the formation of a (stable) interface (Ivanov et al., 2012, Chapter 3). These symbiotic SNARE proteins are highly conserved in dicot plants that host AM fungi and are absent in non-host lineages, which is a strong indication that they are dedicated to symbiosis (Chapter 3 ). The proteins encoded by the symbiosis dedicated SNAREs are not unique in their ability to mediate vesicle fusion to the peri-arbuscular membrane, as the symbiotic SNAREs are functionally interchangeable with their non-symbiotic counterparts. Knock-down of MtSYP132a by RNAi can be complemented by expression of MtSYP132 $\beta$, MtSYP131 or MtSYP121, from the MtSYP132 promoter (Chapters 3 \& 4). Similarly, MtVAMP721d/e knock-down can be complemented by expression of their non-symbiotic family members (Chapter 4). This raises the question why (dicot) plants use dedicated SNAREs for the formation of a host-microbe interface.

In Chapter 4, we have shown that the dominant expression level of symbiotic SNAREs is the main determinant for their essential role in arbuscule formation. However, since the alternative splicing of SYP132 does not affect the total level of SYP132 transcripts, there must be another reason for the conservation of alternative splicing of SYP132 in most dicot plants. Therefore, we hypothesized that SYP132a must have unique symbiosis-dedicated properties, that are lacking in SYP132 $\beta$. These properties are not required for the formation of arbuscules as this can also be mediated by SYP $132 \beta$. Instead, we hypothesize that the unique properties of SYP132a are important at later stages of arbuscule development, for example to control its lifetime or functionality.

The complementation of the SYP132a RNAi has proven to be a suitable approach to show the redundancy of SNAREs in arbuscule formation, since the formation of arbuscules is an easy to observe qualitative phenotype (Chapter 4). However, since we hypothesize that the difference between SYP132 $a$ and other t-SNAREs is most relevant after arbuscule formation, the replacement of SYP132a with another SNARE may result in more subtle, quantitative phenotypes. To analyse such phenotypes the use of hairy root transformation is unsuitable, because it introduces variation in RNAi efficiency, transgene expression and hormone balance of transformed roots. This can introduce variation in mycorrhization or plant growth that masks subtle phenotypes. Therefore, we investigated the functional differences between the two spliceforms of SYP132 using a stable line in which SYP132a has 
been replaced by SYP132 $\beta$. Using CRISPR-Cas9 genome editing, we generated syp132a-1, a mutant in which all transcripts are exclusively spliced into SYP132 $\beta$. We show that there is no difference in colonization of this mutant by either AM fungi or rhizobia. Furthermore, the morphology and lifetime of arbuscules in this mutant are indistinguishable from wildtype. However, we did observe that the SYP132a-CRISPR line shows a significant reduction in plant growth, especially root growth, in a mycorrhiza-dependent manner. This offers a first explanation for the strict conservation of SYP132a in mycorrhizal host plants. Furthermore, by using fluorescent timer fusions to track the turnover of proteins, we found that the preferential localization of SYP132a to healthy parts of partially collapsing arbuscules is the result of the increased stability or recycling of SYP132a on functional branches. The endocytic turnover of SYP132a was increased upon overexpression of VAMP721e causing an accumulation of SYP132a at the degrading parts of arbuscules. Our data suggest that SYP132a controls arbuscule activity/functionality independent from its role in arbuscule formation, although the mechanistic basis for the symbiosis-specific function of SYP132a remains a topic for further study. 


\section{RESULTS}

\section{Generation of syp132a-1, a constitutively SYP132 $\beta$-splicing mutant.}

To generate a SYP132a knockout line, we designed a CRISPR construct that targets the last exon of SYP132a, which is specific for this isoform. This construct was introduced into the Medicago ecotype R108 through stable transformation and offspring was analysed for mutations in SYP132a. Eight independent transgenic lines expressing the CRISPR-Cas construct were isolated, and screened for mutation in SYP132a. One line was identified that was heterozygous for a mutation in SYP132a representing a 532 bp deletion, which includes the entire a-specific exon as well as $34 \mathrm{bp}$ of the last intron (Figure 1a). In the next generation, plants homozygous for this mutation were selected. Offspring of this line, named syp132a-1, was screened for symbiotic defects during AM symbiosis and rhizobium symbiosis, both of which were previously shown to be severely affected by spliceform-specific RNAi of SYP132a (Chapter 3).
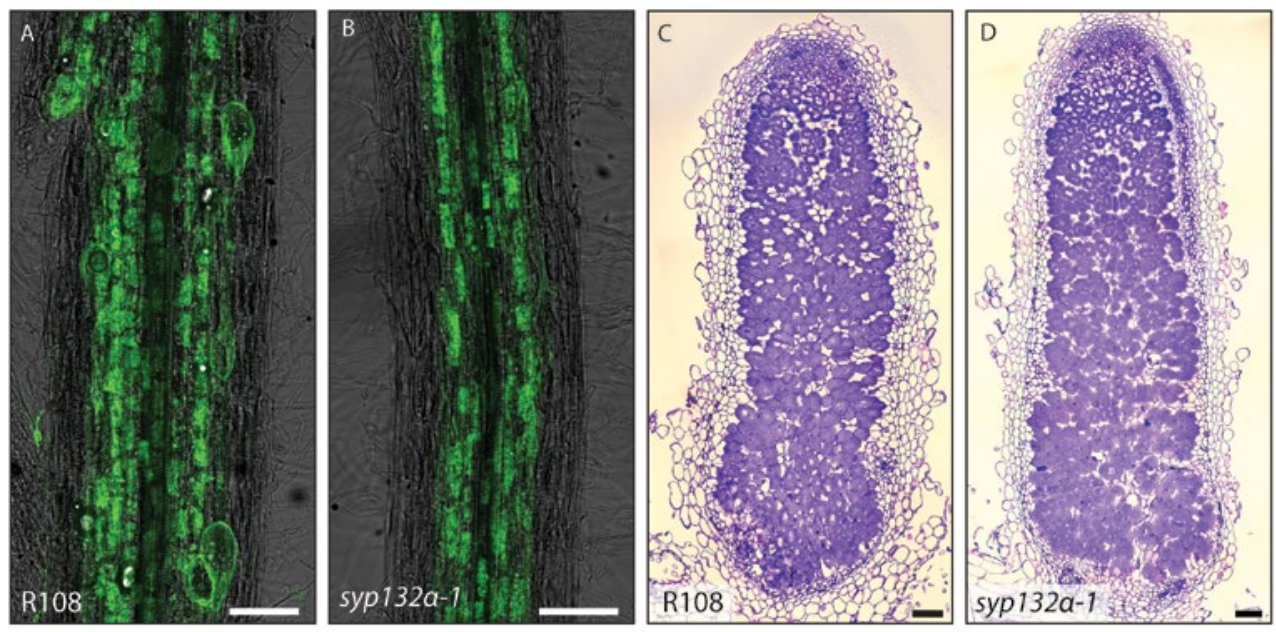

$\mathrm{E}$

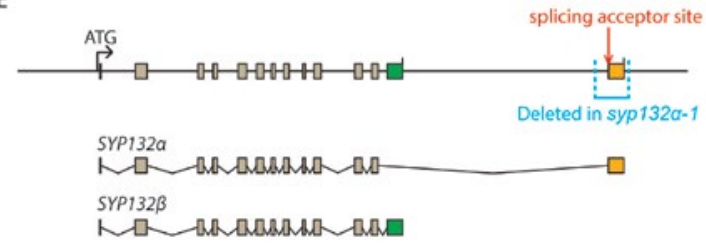

Figure 1.

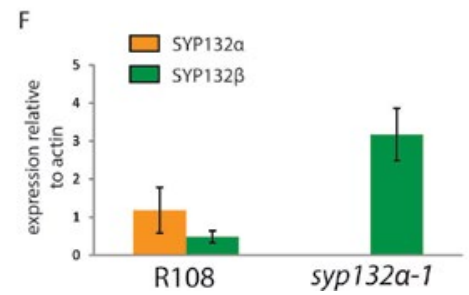

A-B Mycorrhized wildtype (A) and syp132a-1 roots stained with WGA-Alexa488. Scale bars are $100 \mu \mathrm{m}$. D-E Toluidine blue stained sections of nodules on wildtype (C) and syp132a-1 roots. Scale bars are $100 \mu \mathrm{m}$. E Schematic representation of the SYP132 exons in the genome of Medicago, the different spliced transcripts, and the region deleted in the syp132a-1 mutant. $\mathrm{F}$ digital droplet PCR measurements of the transcript levels of SYP132a and $-\beta$ in the arbuscular cells of wildtype plants and syp132a-1 plants. Error bars represent standard deviation of 4 (R108) or 3 (syp132a-1) biological replicates. 
Surprisingly, we did not observe the premature degradation of arbuscules or impaired symbiosome development phenotype previously observed upon silencing of the SYP132a isoform. Instead, the formation of arbuscules and symbiosomes appeared similar to wild-type (Fig. 1a-d).

Since the deletion in this mutant includes the splice acceptor site in front of the SYP132a-specific last exon, we hypothesised that in the syp32a-1 mutant all SYP132 transcripts are eventually spliced into the $\beta$-isoform in absence of the alternative $\alpha$-splicing acceptor site. In such case the lack of SYP132a will be compensated by an equal increase of SYP132 $\beta$ levels. To test for this compensation, we harvested arbuscule containing cells using laser capture microdissection and used digital droplet PCR (ddPCR) to measure absolute transcript levels of both spliceforms in these cells. As shown in figure 1f, the lack of SYP132a in the syp132a-1 mutant is indeed compensated by an increase of SYP132 $\beta$ levels. This suggests that SYP132 $\beta$ can functionally replace SYP132a, in line with the previously reported complementation of SYP132a RNAi through expression of SYP132 $\beta$ (Chapter 3).

\section{syp132a-1 plants remain smaller than wildtype plants when mycorrhized}

The syp132a-1 CRISPR mutant line offered an elegant opportunity to test to which extent SYP132 $\alpha$ and $-\beta$ are truly interchangeable or reveal subtle differences. To study the symbiotic phenotype of syp132a-1 in more detail, we quantified several parameters of mycorrhization, according to Trouvelot et al. (1986). We found that the level of mycorrhization (F\%) of wildtype and syp132a-1 was identical, as well as the number of arbuscules (A\%) (Fig. 2a). In addition, we counted the number of fungal storage vesicles per mycorrhized root length. From this, we found that the number of vesicles formed in syp132a-1 was slightly, but significantly higher than wild-type (Student's t-test, $p \leq 0.05$; Fig. 2b). As a measure of effectiveness of the symbiosis, we measured the phosphate levels of the shoots of mycorrhized plants as described in Novozamsky et al. (1983). The levels of phosphate per dry weight was the same between syp132a-1 and wildtype plants (Fig. 2c). Next, we tested the expression of AM symbiosis marker genes by qPCR. We tested markers for symbiotic signalling (RAM1; Gobbato et al., 2012), arbuscule degradation (MYB1; Floss et al., 2017), and genes involved in fungal or plant nutrition (PT4; Harrison et al., 2002; SWEET7; J. An Unpublished; RAM2, Bravo et al., 2017). None of these showed a significant difference in expression between syp132a-1 and wildtype plants (Fig. 2d).

Although none of our analyses revealed striking differences in morphology or level of mycorrhization, we noted that in all our experiments (4 independent repetitions), the mycorrhized syp 132a-1 mutant plants remained smaller than wildtype control plants (Fig. 2e). The fresh weight of mycorrhized syp132a-1 and wildtype plants was quantified for roots and shoots separately, showing that especially the root biomass of syp132a-1 was severely reduced (Fig. 2f). Although also the average shoot biomass was reduced in all 


\section{Chapter 5}

R108 $\square$ syp132a-1

A

mycorrhization

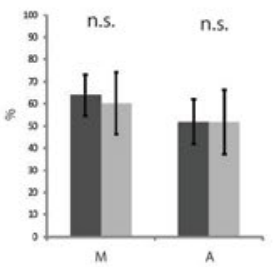

$D^{25}$
B nuber of vesicles

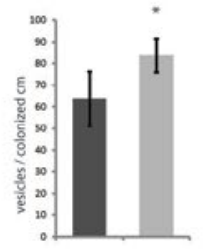

C shoot phosphate content

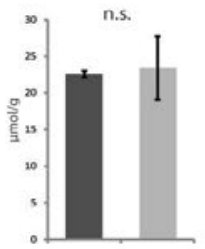

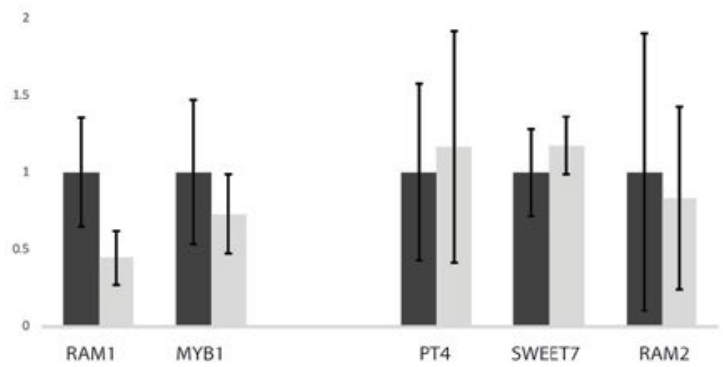

$E$

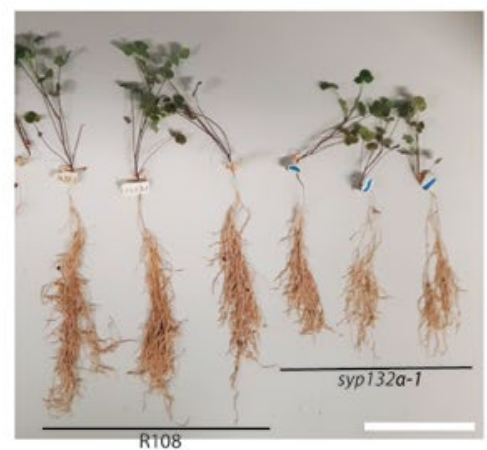

F

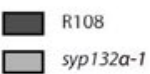

plant fresh weight

syp 132a-1

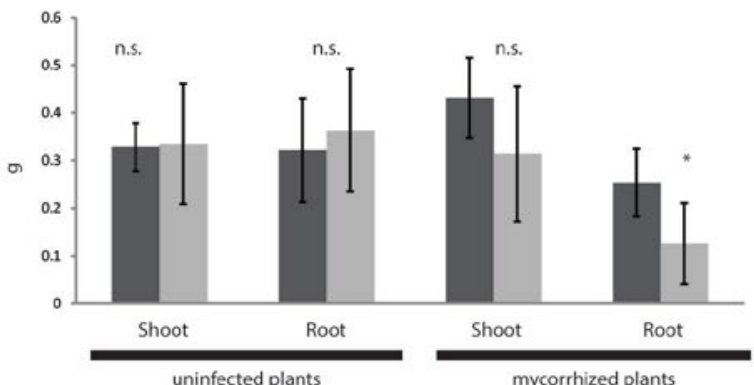

Figure 2

A. Mycorrhization (M\%) and arbuscule abundance (A\%) in wildtype and syp132a-1

roots, 6 weeks after inoculation with

R. irregularis. Parameters are scored according to Trouvelot et al., 1986. Error bars represent the standard deviation of 5 biological replicates B. number of fungal storage vesicles corrected for the mycorrhization levels in A. Error bars represent the standard deviation of 5 biological replicates. $C$. Shoot phosphate content in wildtype and syp132a-1 roots, 6 weeks after inoculation with $R$. irregularis. Error bars represent the standard deviation of 5 biological replicates. D. qRT-PCR on CDNA isolated from mycorrhized in wildtype and syp $132 a-1$ roots, 6 weeks after inoculation with $R$. irregularis. E image showing macroscopic difference between in wildtype and syp $132 a-1$ roots, 6 weeks after inoculation with $R$. irregularis. Scale bar is $5 \mathrm{~cm}$. F. Fresh weights of plants 6 weeks after inoculation with $R$. irregularis, and non-inoculated control plants. A-D, F. n.s. indicates non-significant differences, asterisk indicates significant difference (Student's t-test $\mathrm{p} \leq 0.05$ ). 
repetitions of the experiment, this was not always significant. The effect on plant growth was dependent on mycorrhizal colonization, as no significant difference between the wild-type R108 and syp132a-1 plants was observed in the absence of mycorrhiza. This suggested a symbiotic deficiency in the syp $132 a-1$ plants which prompted us to examine arbuscule development in the mutant line in more detail.

\section{Arbuscule development in syp 132a-1}

Since the biomass of mycorrhized syp132a-1 plants was lower than wildtype, we suspected that the functionality or lifetime of the arbuscules was reduced in the mutant. To test whether specialized peri-arbuscular membrane cargo is still able to reach the peri-arbuscular membrane in the mutant, we analysed the localization of the symbiotic phosphate transporter PT4 fused to GFP expressed from its own promoter (Pumplin \& Harrison, 2009). In both mutant and wildtype plants it localized to the tips of the growing arbuscule with no apparent difference in intensity (Fig. 3a and b). Since a previous study on arbuscule lifetime found subtle differences in the size distribution of arbuscules (Floss et al., 2017), we also determined the arbuscule size distribution in the syp132a-1 mutant, by measuring the arbuscule size in images of 1000 arbuscules per root of wildtype and mutant plants $(n=4)$. This revealed no differences in arbuscule size distribution (Fig. $3 c$ ). From the same images we quantified the fraction of collapsed arbuscules, which was not significantly different. To get more direct data on the lifetime of arbuscules, we developed a construct that expresses the fluorescent timer reporter protein dsRed-E5 fused to a nuclear localization tag from the PT4 promoter. DsRed-E5 slowly maturates from green to red fluorescence with a half-time of approximately 10 hours (Mirabella et al., 2004). Since the PT4 promoter is switched on precisely at the start of the formation of the peri-arbuscular membrane (Pumplin et al., 2012), the ratio of red/green fluorescence can be used as a proxy for arbuscule age. We expressed the PPT4::Timer-NLS construct in mycorrhized Medicago roots and determined the red/green ratio of the nucleus of approximately 100 arbuscular cells per root $(n=4)$. We observed a clear correlation between the colour of the nucleus and the phenotype of the arbuscule: Arbuscules with young (turgoid) branches that did not yet completely fill the cell displayed a low nuclear red/green ratio, while collapsed arbuscules displayed a high nuclear red/green ratio. Mature arbuscules displayed intermediate red/green ratio's (Fig. 3d). This confirmed the suitability of the construct to measure arbuscule age. The age distribution of arbuscule cells in syp132a-1 plants was not significantly different from the age distribution in wildtype plants (Fig. 3e). In summary, despite the observed effect on plant growth, these analyses did not reveal any effect on arbuscule morphology or lifetime in the syp132a-1 mutant line. 

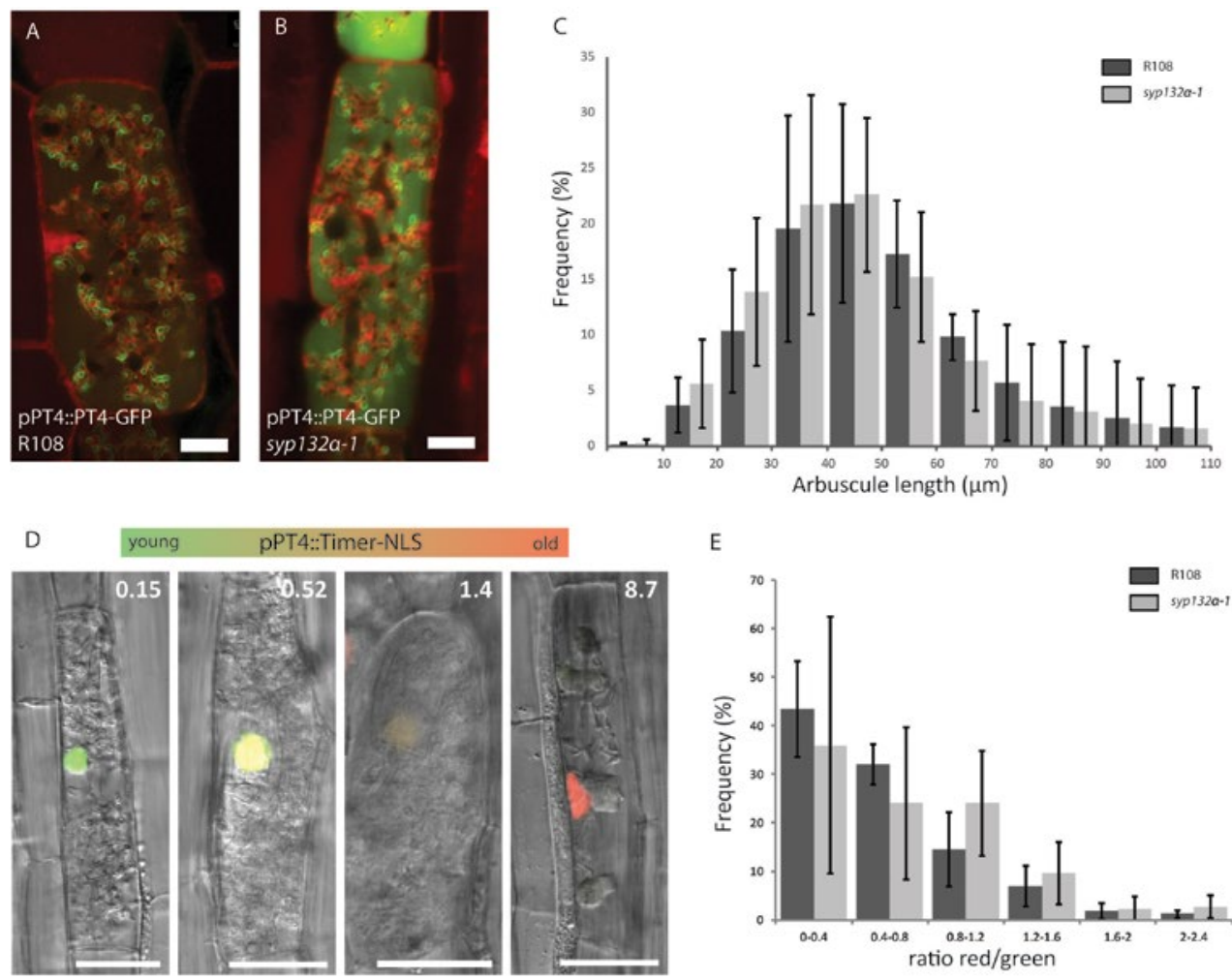

\section{pPT4::Timer-NLS}

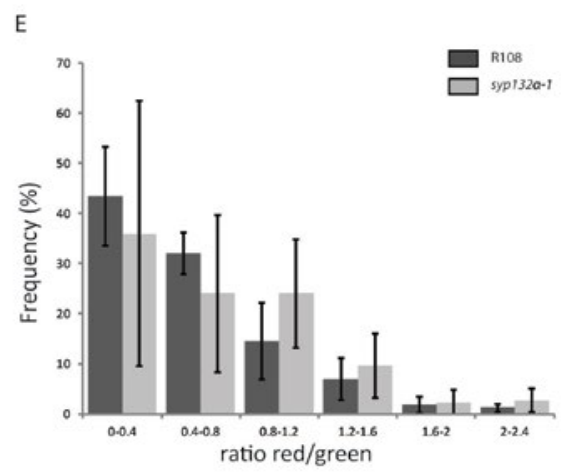

Figure 3.

A-B Confocal laser scanning microscopy images showing localisation of PHOSPHATE TRANSPORTER 4 (PT4) labelled with GFP driven by its native promoter in arbuscular cells of wildtype and syp132a-1 roots. The cytoplasm and nuclei are labelled with dsRed driven by the Arabidopsis ubiquitin 3 promoter. C. Arbuscule length distribution measured in wildtype and syp132a-1 roots infected with R. irregularis. Around 1000 arbuscules per root were measured. Error bars represent standard deviation of 4 different roots. D. Confocal laser scanning microscopy images overlaid with DIC images showing expression of nuclear localized dsRed-E5 (Timer) driven by the PT4 promoter in arbuscular cells of wildtype plants. The numbers in the upper-right corner indicate the ratio between red and green fluorescence. E. Age distribution of arbuscules in wildtype and syp132a-1 roots. The age of 100 arbuscules was measured per root. Collapsed arbuscules were not measured. Error bars represent standard deviation of 4 individual roots. $C$ and $E$ no significant differences between wild-type and syp132a-1 were found (Student's t-test $p \leq 0.05$ ).

\section{SYP132 isoforms show a differential turn-over upon arbuscule collapse}

The reduced biomass of mycorrhized syp132a-1 plants suggests that there is a functional difference between SYP132 $\alpha$ and SYP132 $\beta$. Previously, we observed a difference in localization between the SYP132 isoforms when arbuscules start to collapse, with SYP132a being more confined to the non-degrading ("healthy") arbuscule branches and SYP132 $\beta$ accumulating on degrading parts (Huisman et al., 2016; Chapter 3). Since polar localization of proteins to different parts of the plasma membrane can be the result of directional secretion and/or selective recycling (tangowski et al., 2016), we investigated 
the relative stability of both SYP132 isoforms on different arbuscule branches by fusing them to the fluorescent timer dsRed-E5, driven from the arbuscule-specific PT4 promoter. To correct for the time the PT4 promoter is active in the cell, we simultaneously expressed dsRED-E5 fused to a nuclear localization signal driven by the same promoter. Before arbuscules collapse, both isoforms displayed identical behaviour: The red/green ratio on arbuscular branches was more shifted to red compared to the signal on the tips of growing arbuscules (Fig. 4a; only SYP132 $\beta$ shown). This indicates that both isoforms are delivered preferentially to the tip of growing arbuscules. When the arbuscules collapse partially, this pattern changes; the collapsed part is labelled mostly by red (older) proteins, whereas the 'healthy' turgoid parts are mostly green/young. At this stage the healthy arbuscule branches appeared to be evenly labelled, with no difference between tips and older branches. Both SYP132 isoforms showed a similar pattern. (Fig. 4b-d). Strikingly, the healthy part of partly collapsing arbuscules showed a lower red/green ratio than the arbuscule branches of mature arbuscules before collapse, as a result of lower levels of red fluorescence (Fig. $4 \mathrm{~d}, \mathrm{f}$ ). This indicates that more older/red proteins are being removed at this stage. The removal of old/red proteins from the healthy parts of collapsing arbuscules coincides with a stronger red labelling of the collapsed parts, suggesting that such proteins are recycled from the healthy to the collapsed part. Both SYP132 $\alpha$ and $-\beta$ eventually label the collapsed parts (Fig 4e-f). However, we observed a difference in the absolute levels of both isoforms on the healthy branches of collapsing arbuscules; the amount of red fluorescent SYP132a is significantly higher than observed for SYP132 $\beta$ on these branches (Fig. 4f). Also the amount of green/young SYP132a seemed to be higher, although this difference was not significant (Fig. 4e). These observations suggest that the earlier observed difference in localization of both isoforms in partly collapsing arbuscule cells (Chapter 3 ) is not the result of a difference in delivery of newly formed protein, but the result of slower turn-over/re-localization of SYP132a to the collapsed part compared to SYP132 $\beta$, or a preferential recycling of SYP132a back to the healthy domains.

Strikingly, when we co-expressed SYP132a with VAMP721e both from the PT4 promoter (Chapter 4), we noticed that SYP132a localized mostly to the collapsed part of partly degrading arbuscules, whereas it was nearly absent from the healthy parts (Fig. $4 \mathrm{~g}$ ). This localization was observed in 13 out of 13 partially collapsing arbuscules, whereas without overexpression of VAMP721e, SYP132a preferentially localized to the healthy branches in 28 out of 29 partially collapsing arbuscules (Fig. 4h). This indicates that overexpression of VAMP721e causes the re-localization of SYP132a towards collapsing arbuscule domains. 


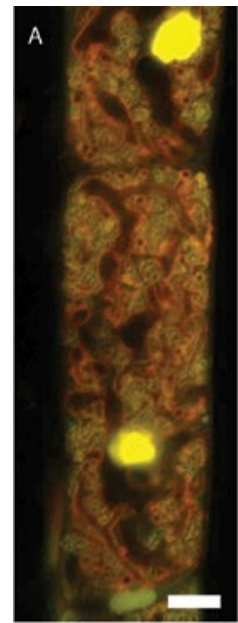

pPT4::Timer-SYP132 PPT4::Timer-NLS

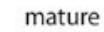
arbuscules

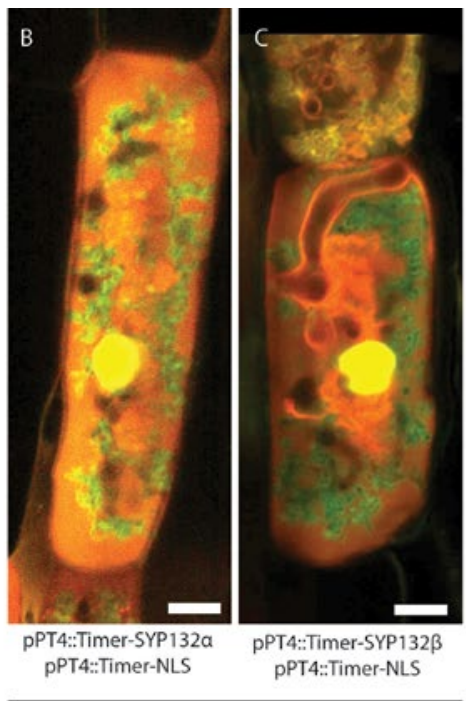

partially collapsing

arbuscules
D SYP132 age $=\frac{\mathrm{red} / \text { green membrane }}{\mathrm{red} / \text { green nucleus }}$

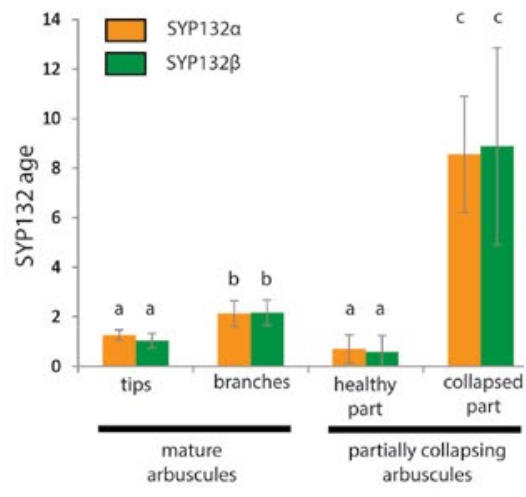

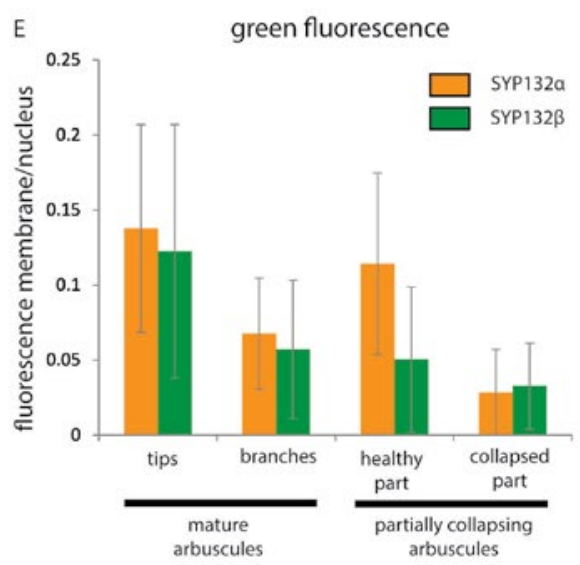
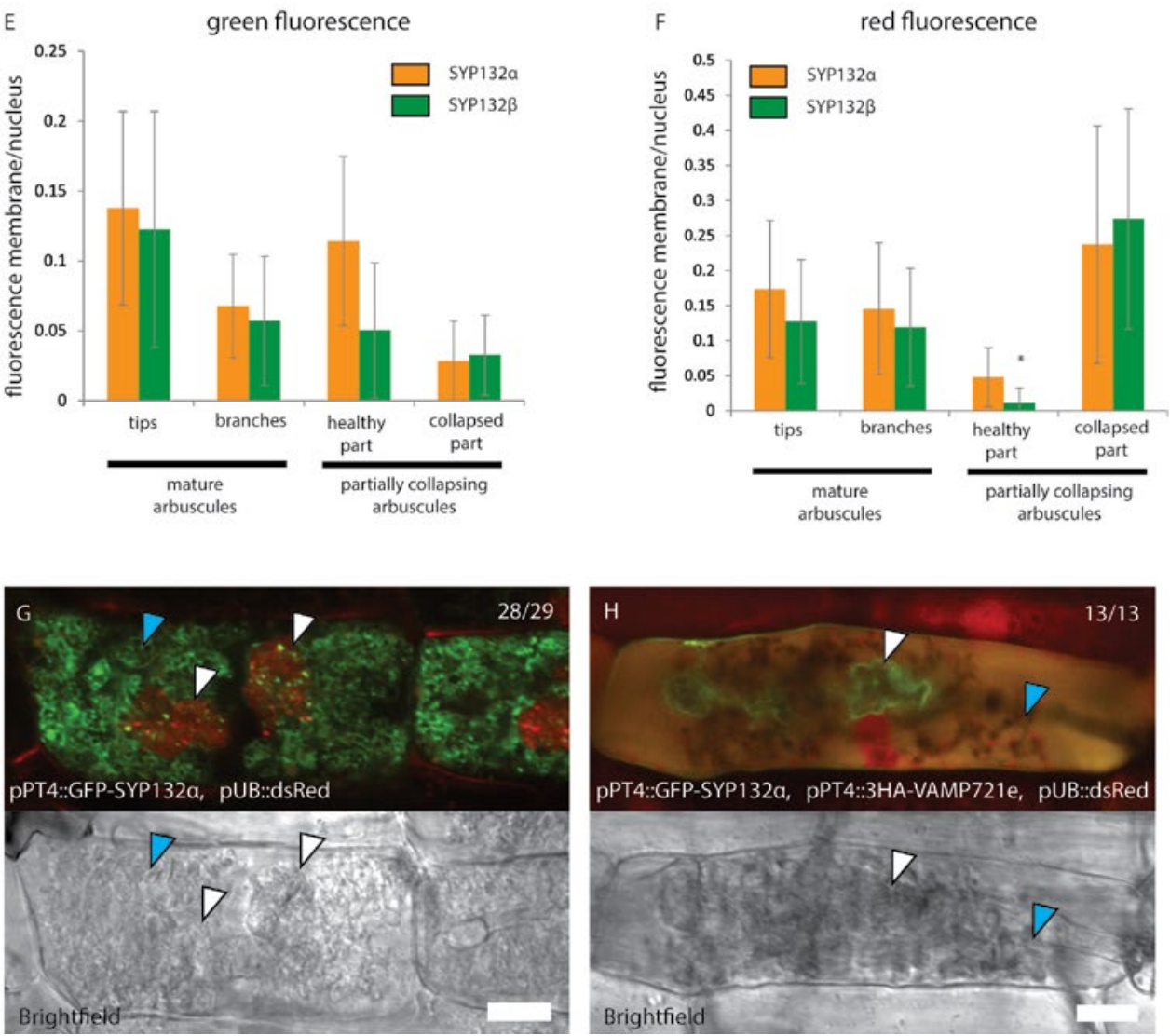


\section{DISCUSSION}

The evolutionary conservation of the SYP132a isoform in mycorrhizal-host plants, suggested that this isoform has a symbiosis-specific function. Here, we have studied the functional differences between SYP132 $\alpha$ and $-\beta$ by generating syp 132 $\alpha-1$, a constitutive $\beta$-splicing mutant, and by studying the difference in dynamics between the two proteins. We have shown that although both SYP132 $\alpha$ and SYP132 $\beta$ are sufficient for the formation of arbuscules, the biomass of mycorrhized syp132a-1 is severely reduced, suggesting that these arbuscules are functionally impaired. Despite extensive investigations, we did not observe any difference in the morphology or lifetime of the arbuscules compared to wildtype. Therefore, the syp132a-1 mutant appears to uncouple arbuscule morphology from function, as discussed below.

Our initial goal in generating a syp132a mutant through CRISPR-Cas9 technology was to knock out SYP132a without affecting the SYP132 $\beta$ levels, resembling the specific RNAi of SYP132a (Chapter 3). Such mutant could be used to knock-in different non-symbiotic SYP1 paralogs. Instead, we obtained the syp132a-1 mutant where the absence of SYP132a transcripts is compensated by the increased levels of SYP132 $\beta$ transcripts. In this respect, the syp 132a-1 mutant resembles the SYP132a RNAi plants complemented with SYP132 $\beta$ (fig. 6 of Chapter 3). In this previous experiments, the observed complementation could still be attributed to the partial knockdown of SYP132a in combination with an approx. 10-fold overexpression of SYP132 $\beta$. However, the absolute expression levels of both isoforms in arbuscule cells of syp132a-1 and wildtype plants show that the compensation of SYP132a knockout by SYP $132 \beta$ does not rely on its overexpression. The fact that during nodulation syp $132 a-1$ is also not impaired in symbiosome release or differentiation further shows that both isoforms are largely interchangeable in rhizobium symbiosis.

\section{Figure 4.}

A-C. Confocal laser scanning microscopy images showing SYP132 $\alpha$ and $-\beta$, fused to dsRed-E5 (Timer), co expressed with nuclear localized Timer, both driven by the PT4 promoter. The brightness of the red and green channels in all three images were normalized against the red and green levels in the nuclei, to allow comparison between images. Note, in this figure, nuclei were overexposed to visualize weaker Timer-SYP132 fusion proteins, but care was taken not to saturated raw images used for the analyses. D-F. Quantification of red and green florescence on different subcellular regions of mature and partially collapsing arbuscules expressing Timer-SYP132 fusions. Error bars represent standard deviation of 15 (SYP132a, mature) 7 (SYP132a, collapsed) 13 (SYP132 $\beta$, mature) or 18 (SYP132 $\beta$, collapsed) cells. Different letters or asterisk indicate significant difference (Student's t-test $\mathrm{p} \leq 0.05)$. G-H. Confocal laser scanning microscopy images and accompanying bright-field images showing the localization of SYP132a fused to GFP without $(\mathrm{G})$ and with $(\mathrm{H})$ co-expression of triple HA-tag labelled VAMP721e, both driven by the PT4 promoter. Collapsed branches are marked by white arrowheads, healthy branches are marked by blue arrowheads. The red signal shows autofluorescence of collapsed arbuscules and the nuclei and cytoplasm that are labelled with dsRed driven by the Arabidopsis ubiquitin 3 promoter. Scale bars are $10 \mu \mathrm{m}$. 
We found that the symbiotic phosphate transporter PT4 is expressed in syp $132 a-1$ mutants and delivered to the peri-arbuscular membrane as in wild-type roots. Further, similar phosphate levels were measured in the leaves of mycorrhized syp132a-1 and wild-type plants. Therefore, the mycorrhiza-dependent reduction in plant growth in the mutant is unlikely to be caused by a reduction of phosphate transfer to the plant. Interestingly, we noticed a higher amount of fungal storage vesicles in the syp132a-1 roots, which might indicate a difference in the amount of carbon transferred to the fungus.

Which biological process is impaired in the syp132a-1 mutant is a major question that still remains, however the delivery of membrane to arbuscules in this mutant appears to be sufficient to support their growth to full maturation. Since SYP132a does not selectively interact with specific v-SNAREs to mediate the fusion of a specific population of vesicles (Chapter 4), it can be assumed that the same vesicles mediate the growth of the peri-arbuscular membrane in syp132a-1 mutant plants and wild type plants. Therefore, the specific use of SYP132a seems to be independent of its role in vesicle fusion. A potential explanation for the specialization of SYP132a could involve an interaction with specific peri-arbuscular membrane localized transporters. Such a scenario is not without precedent. For example, the t-SNARE AtSYP121 was shown to interact with $\mathrm{K}^{+}$-channels KC1 and KAT1. As a result of this interaction, $\mathrm{K}^{+}$uptake is reduced in Atsyp121 mutants (Grefen et al., 2010). Also in animal systems, syntaxins have been shown to interact with and directly regulate a range of ion channels or transporters (Michaelevski et al., 2003; Chao et al., 2011). Interaction of SYP132a with nutrient transporters on the peri-arbuscular membrane may affect the nutrient exchange, leading to impaired root growth in the syp132a-1 mutant.

Previously, we found that SYP132a and $-\beta$ differentially mark healthy and degrading arbuscule branches, when arbuscules start to collapse (Chapter 3 ). Based on this, we hypothesized that SYP132 a mediates vesicle transport to the healthy parts, while SYP132 $\beta$ could mediate vesicle transport that allows a cell to degrade arbuscules and return to a non-symbiotic state. However, this hypothesis did not take into account the encasement-like character of the membranes surrounding the collapsed arbuscular branches. From transmission electron microscopy images, it was shown that collapsed arbuscule branches are encased by depositions of cell-wall like material, with many paramural bodies (Cox \& Sanders, 1974). Such encasement is highly similar to the encasement of haustoria from filamentous pathogens, which occur as result of a defence response towards these pathogens. During the encasement of haustoria multivesicular bodies (MVBs), which normally fuse to the vacuole to target membrane proteins for degradation, are redirected towards the haustorial encasement. This results in the inclusion of the inner vesicles of MVB within the deposited cell-wall material as exosomes (Assaad et al., 2004; An et al., 2006; Micali et al., 2011). Following this route, membrane localized proteins including the t-SNARE SYP121 accumulate in the encasement (Nielsen et al., 2012). Since 
the SYP121 SNARE domain will be on the inside of the exosomes, accumulation of SYP121 at encasements does not reflect a specialized role in vesicle fusion to the peri-haustorial membrane(Meyer et al., 2009; Nielsen \& Thordal-Christensen, 2013). Consistent with an encasement-like arbuscule degeneration process, the localisation of SYP132 on the collapsing parts of arbuscules appeared to be wider and more irregular and included fluorescent signal in the middle of the clumps indicating localisation to paramural bodies (see for example Fig $4 \mathrm{H}$ ). Therefore, similar to the accumulation of SYP121 in the encasement of haustoria, the presence of SYP132 on collapsing arbuscules may represent a retargeting of MVB's containing endocytosed syntaxins to the PAM-encasement (Figure 5). In this scenario, the biologically relevant difference between SYP132a and $-\beta$ is their presence on the healthy branches, not their different accumulation on the collapsing branches.

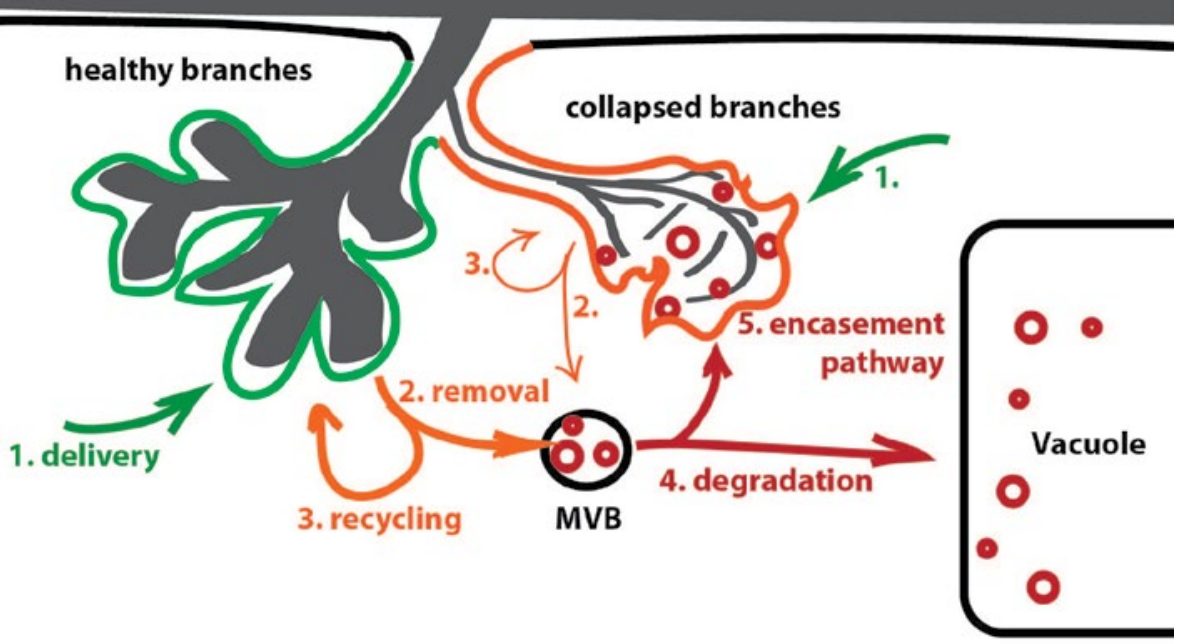

\section{Figure 5. Model predicting SYP132 dynamics during arbuscule collapse}

Colours indicate the average age of SYP132, from young (green), to intermediate (orange) to old (red). 1. Newly synthesized proteins are delivered to both functional and collapsed branches. 2. Proteins are removed from the membrane by endocytosis. After endocytosis, they can be recycled to the peri-arbuscular membrane (3), or enter the endosomal pathway for degradation. The net removal of SYP132a from the healthy branches is lower than SYP132 $\beta$. This can be due to lower endocytosis, or due to increased recycling. The average age of SYP132 on the collapsed branches is higher than on the healthy branches. This may be due to a lower removal rate from collapsed branches. 4. Membrane proteins that are targeted for degradation are translocated to the inner vesicles of multi vesicular bodies (MVBs) that fuse with the vacuole. 5 . The lytic degradation and encasement of collapsed arbuscules may involve the re-direction of MVBs bodies to the collapsed branches. This results in the presence of SYP132 labelled exosomes in the encasements, that contribute to the high average age of SYP132 on collapsed branches. 
The use of fluorescent timer constructs allowed us to monitor the relative age of arbuscules and associated SYP132 proteins at different parts of the arbuscules. We observed that the age of SYP132 proteins on the collapsed arbuscule branches was much older than on the healthy parts. This pattern holds true for both spliceforms of SYP132. It suggests that both isoforms are endocytosed from the healthy parts, while at the collapsed parts they are more stationary or recycled back after endocytosis. The stationary presence of proteins at the collapsed part could be the result of their presence within the encasement, as described above, from which they cannot be removed. These analyses further revealed a difference in the dynamics of the SYP132 isoforms upon collapse; The amount of SYP132a at the healthy branches was higher compared to SYP132 $\beta$, indicating a slower turn-over or enhanced recycling of SYP132a compared to SYP132 $\beta$. As a consequence, we expected the average age of SYP132a on healthy branches to be older than SYP132 $\beta$. However, the half-life of the timer may be insufficient to show these differences. A model to explain the different behaviour of the SYP132 isoforms during arbuscule collapse is shown in Figure 5.

Interestingly, when we overexpressed VAMP721e together with SYP132a, the turn-over of SYP132a became similar to SYP132 $\beta$. We previously showed that significantly more VAMP721e protein is found in SNARE complexes with SYP132 $\beta$ than with SYP132a, which suggested that SYP132a-containing cis-SNARE complexes were more rapidly disassembled (Chapter 4). Since VAMP721e also accumulates on the encasement (not shown), the endocytotic turn-over of both SYP132 isoforms may be enhanced by their interaction with VAMP721e. Therefore, the difference in localisation between the two SYP132 isoforms may be the result of differences in cis-SNARE complex stability. The different behaviour of SYP132 $a$ and SYP132 $\beta$ is most apparent when the arbuscule collapses partially, but a difference in protein/SNARE complex stability on the peri-arbuscular membrane may be of biological relevance already before the collapse of arbuscules. The fact that a difference in protein localization is only observed during collapse of arbuscules may be due to the use of the PT4 promoter to drive the expression of the fusion-proteins. The PT4 promoter is switched off when arbuscules collapse (Volpe et al., 2016), allowing visualisation of protein stability without interference from newly synthesised proteins at this stage.

A difference in protein stability between the two SYP132 isoforms may also occur at the symbiosome membrane during rhizobium symbiosis. Until now, there has been some controversy related to the differential localization of the two SYP132 spliceforms in nodule cells. We reported that both SYP132 spliceforms equally localize to symbiosomes and the plasma membrane (Chapter 3). In contrast, Pan and co-workers reported that SYP132 $\beta$ strictly localized to the plasma membrane without labelling symbiosomes and SYP132a mainly localized to symbiosomes (Pan et al., 2016). Based on these findings, Pan and co-workers hypothesized that SYP132 $\alpha$ and $-\beta$ separate traffic to the symbiosomes and plasma membrane. Both their and our studies made use of artificial promoters; we 
used the Arabidopsis EF1 $a$ and pea Leghaemoglobin promoters, while Pan and co-workers used the Medicago DNF1 promoter. The DNF1 promoter is switched off in the fixation zone (Wang et al., 2010a). Thus, if SYP132a is more stable on symbiosomes compared to SYP132 $\beta$, it may remain on differentiated symbiosomes for a longer time after it's expression has been shut down, while SYP132 $\beta$ may be removed much faster. This difference in stability is not observed using the EF1a and Leghaemoglobin promoters, which remain active during symbiosome development (Auriac \& Timmers, 2007; Limpens et al., 2009). Nevertheless, these promoters show that there is no difference in targeting of the two isoforms to the symbiosomes. The observation that SYP132 $\beta$ can compensate for the loss of SYP132a in syp132a-1 nodules shows that the two spliceforms do not separate traffic to the symbiosomes and the plasma membrane.

Summarizing, our results indicate that SYP132a is dedicated to symbiosis and required for full arbuscule functionality, explaining its conservation in all AM-host plants after its evolution. Although our results do not yet reveal the mechanism(s) by which SYP132a performs a symbiosis-dedicated function, the insights obtained here offer a starting point for future investigations that may reveal new aspects of arbuscule maintenance that are required for efficient nutrient transfer.

\section{MATERIALS AND METHODS}

\section{Plasmid construction}

To make a CRISPR-Cas9 construct for SYP132a we made use of the PCAMBIA1302-Cas9 vector described by Jiang et al. (Jiang et al., 2013). A 20 bp region (GCAACTGATCATGTGAAGTC) targeting the last exon of SYP132a was chosen as sgRNA target sequence. Overlap PCR was performed to introduce the selected 20 bp target sequence into the U6-sgRNA cassette from pCAMBIA1302-Cas9, flanked by Sall and Kpnl restriction sites, using the primers 1-4 (Table S1). The resulting SYP132a-sgRNA fragment was introduced into the $p C A M B I A-C A S 9$ vector via Sall-Kpnl restriction-ligation. pCAMBIA-CAS9 additionally contains the CAS9 gene under the control of the CaMV35 promoter and a hygromycin resistance gene for selection in the plant.

A pPT4::PT4 GFP expression vector was provided by Prof. Maria Harrison and described in (Pumplin \& Harrison, 2009). This expression cassette amplified by PCR, using primers 29 and 30 (table S1) adding Bsal restriction sites that generate a Bsp120l compatible sticky overhang. Using Bsal-Bsp120l restriction digestion, the PCR fragment was inserted into an empty binary RNAi vector carrying cytoplasm marker dsRed driven by the pUB promoter described in (Huisman et al., 2016).

All other expression cassettes were constructed using multisite gateway technology (Invitrogen) in the pKGW-MGW destination vector, using a pENTR2-3 carrying a $35 \mathrm{~S}$ 
terminator (Ovchinnikova et al., 2011). For this, a pENTR4-1 vector carrying a PT4 promoter, a pENTR4-1 carrying a PT4 promoter fused to GFP, and pENTR1-2 vectors carrying the coding sequences of SYP132 $\alpha$ and $-\beta$ were described in (Huisman et al., 2016). a pENTR1-2 carrying a Timer-NLS construct was generated by amplifying the timer-NLS cds from a vector described in (Mirabella et al., 2004), using primers 31 and 32 (Table S1) adding a cacc sequences in the forward primer. The PCR fragment was then cloned into a pENTR/D-TOPO entry vector using TOPO cloning (Invitrogen). A pENTR4-1 carrying the fluorescent timer driven by the PT4 promoter was constructed by amplifying the timer cds using primers 33 and 34 (Table S1), while adding Ascl-Kpnl restriction sites. By Ascl-Kpnl restriction digestion, the GFP of the pENTR4-1 pPT4-GFP was swapped for Timer. The Timer-SYP132 fusions were combined with the Timer-NLS constructs, by amplifying the Timer-NLS expression cassette using primers 35 and 36 (Table S1), while adding Apal and Eco81I restriction sites. Using Apal-Eco81I restriction digestion, the cassete was then inserted into the binary vectors already carrying the Timer-SYP132 fusion constructs.

\section{Stable transformation of Medicago truncatula}

The binary plasmid carrying SYP132a-sgRNA/35s::CAS9 construct the was introduce into Agrobacterium tumefaciens AGL1 via electroporation. Stable transformation of Medicago truncatula R108 cotyledon and young leaf explants was done according to Chabaud et al. (Chabaud et al., 2003). Transformants were selected using $10 \mathrm{mg} / \mathrm{L}$ hygromycin B. DNA was extracted from the transformed lines using the standard CTAB miniprep method. The resulting lines were genotyped, and resulting PCR amplicons sequenced, using the primers 5 and 6 (Table S1). The presence of the Cas9 gene in the obtained lines was checked by PCR using primers 7 and 8 (Table S1).

\section{Plant growth, Transient transformation and inoculation}

For transformation, Agrobacterium rhizogenes MSU440 was used according to (Limpens et al., 2004). For nodulation assays, plants were transferred to perlite saturated with Färhaeus medium without $\mathrm{Ca}\left(\mathrm{NO}_{3}\right)_{2}$ and grown at $21^{\circ} \mathrm{C}$ at a $16 / 8 \mathrm{~h}$ light/dark regime. After three days, plants were inoculated with Sinorhizobium melilotii 2011 and grown for 4 weeks. For mycorrhization assays, plants were transferred to pots containing a 5:3 (v/v) ratio mix of expanded clay and sand, saturated with modified Hoagland medium (5 mM MgSO ${ }_{4^{\prime}} 2.5$ $\mathrm{mM} \mathrm{Ca}\left(\mathrm{NO}_{3}\right)_{2}, 2.5 \mathrm{mM} \mathrm{KNO}_{3}, 2 \mathrm{mM} \mathrm{NH}_{4} \mathrm{NO}_{3}, 500 \mu \mathrm{M}$ MES, $50 \mu \mathrm{M}$ NaFeEDTA, $20 \mu \mathrm{M} \mathrm{KH}_{2} \mathrm{PO}_{4}$ 12,5 $\mu \mathrm{M} \mathrm{HCl}, 10 \mu \mathrm{M}$ H3BO3, $2 \mu \mathrm{M}$ MnCl2, $1 \mu \mathrm{M}$ ZnSO4, $0.5 \mu \mathrm{M}$ CuSO4, $0.2 \mu \mathrm{M}$ Na2MoO4, $0.2 \mu \mathrm{M} \mathrm{CoCl}_{2}, \mathrm{pH}$ 6.1). Plants were inoculated with dried Rhizophagus irregularis infected maize roots obtained from Plant Health Cure (http://www.phc.eu). Plants were grown for four weeks at $21^{\circ} \mathrm{C}$ at a $16 / 8 \mathrm{~h}$ light/dark regime. 


\section{Laser capture microdissection and ddPCR}

Roots of mycorrhized Medicago plants and uninfected control plants were harvested and fixed in Farmer's fixative (75\% ethanol, $25 \%$ acetic acid) substituted with $0,01 \%$ Chlorazol Black E to stain AM fungi, and vacuum infiltrated for 30 minutes on ice. Then, the roots were incubated in Farmer's fixative for 16 hours at $4^{\circ} \mathrm{C}$ on a spinning wheel. After fixation, the roots were dehydrated in an ethanol dehydration series $(80 \%, 85 \%, 90 \%, 95 \% 30$ minutes each followed by $100 \%$, overnight) Steedman wax was prepared by mixing $90 \%$ polyethylene glucol 400 distearate and $10 \% 1$-hexadecanol at $65^{\circ} \mathrm{C}$. Steedman wax was infiltrated by incubating the roots in $50 \%$ Steedman wax and $50 \%$ ethanol for two hours at $38^{\circ} \mathrm{C}$, followed by 3 incubations in $100 \%$ Steedman wax for 2 hours at $38^{\circ} \mathrm{C}$. Finally, the samples were transferred to room temperature to allow the wax to solidify. Solidified blocks of Steedman wax were cut into $20 \mu \mathrm{m}$ thick sections using a microtome, and transferred to PEN-membrane slides (Leica). Arbuscule containing cells and uninfected cortical cells were harvested using a Leica LMD7000 laser capture microdissection microscope. RNA was isolated using a RNeasy micro kit (Qiagen). cDNA was synthesized using the iScript CDNA synthesis kit (Bio-Rad), in a total volume of $10 \mu \mathrm{l} .1 \mu \mathrm{l} \mathrm{CDNA}$ was then used per ddPCR reaction. For this, a ddPCR mastermix containing evaGreen as a probe was used (BioRad), as well gene specific primers (9-14, Table S1). Then the reaction mix was suspended in oil using the QX200 Droplet Generator (Biorad). The PCR was carried out following manufacturer's instructions. Subsequently, the absolute number of positive droplets was counted using a QX200 Droplet Reader.

\section{RNA isolation and qRT-PCR}

RNA was isolated from plant tissue using the EZNA Plant RNA mini kit (omega). CDNA was synthesized from $1 \mu \mathrm{g}$ of RNA using the iScript CDNA synthesis kit (BioRad). Equal amounts of CDNA were used for qPCR using SYBR green supermix (Bio-Rad) in a Bio-Rad CFX connect real-time system qPCR machine. Gene expression levels were determined using gene specific primers 13-26 listed in table S1. The gene expression was normalized using Actin2 and Ubiquitin 10 as reference genes.

\section{Mycorrhizal staining and quantification of mycorrhization}

For quantification of mycorrhization, roots were incubated in $10 \%(\mathrm{w} / \mathrm{v}) \mathrm{KOH}$ at $98{ }^{\circ} \mathrm{C}$ for 10 minutes. Then roots were washed 3 times with $5 \%$ acetic acid. After washing, the roots were stained in $5 \%$ ink in $5 \%$ acetic acid, for 2 minutes at $98{ }^{\circ} \mathrm{C}$. after staining the roots were destained in $5 \%$ acetic acid, refreshing the destaining solution several times. For staining with WGA alexafluor 488 , roots were incubated in $10 \%(\mathrm{w} / \mathrm{v}) \mathrm{KOH}$ at $60^{\circ} \mathrm{C}$ for 3 hours. Then, roots were washed 3 times in PBS $(150 \mathrm{mM} \mathrm{NaCl}, 10 \mathrm{mM} \mathrm{Na} 2 \mathrm{HPO} 4,1.8 \mathrm{mM}$ $\mathrm{KH} 2 \mathrm{PO} 4, \mathrm{pH} 7.4$ ), and incubated in $0.2 \mu \mathrm{g} / \mathrm{mL}$ WGA-Alexafluor 488 (Molecular Probes) in $\mathrm{PBS}$ at room temperature for 16 hours. For quantification of mycorrhization, roots were cut into $1 \mathrm{~cm}$ fragments, and the colonization and arbuscule abundance was scored and calculated according to Trouvelot et al. (Trouvelot et al., 1986). 
Chapter 6

General Discussion 
During arbuscular mycorrhiza (AM) symbiosis and rhizobium symbiosis, the microbial partner is hosted within plant cells, where it is surrounded by a plant derived membrane. This peri-microbial membrane forms the host-microbe interface that is highly specialized for the bidirectional transport of carbon and nutrients across the membrane.

In this thesis, I have studied how plant membrane trafficking is regulated to create a symbiotic host-microbe interface. Previously, it was shown that the formation of a symbiotic host microbe interface during both of these symbioses depends on the vesicle SNAREs (v-SNAREs) VAMP721d and -e (Ivanov et al., 2012). These SNAREs are embedded in the VAMP72 gene family of v-SNAREs, which are required for exocytosis (Lipka et al., 2007). This shows that the formation of a symbiotic host-microbe interface depends on an exocytosis-like membrane trafficking pathway, that is shared between AM symbiosis and rhizobium symbiosis. Furthermore, it shows that the host-microbe interface is a subdomain of the plasma membrane. For simplicity, I will use the term plasma membrane to describe the non-symbiotic domain which surrounds the (host) cell. The genomes of most dicot plants that can host AM fungi contain an ortholog of MtVAMP721d/e. In contrast, most plants that lost the ability to interact with AM fungi like Arabidopsis have lost their copy of this gene. (Ivanov et al., 2012; Chapter 4). Genes that are essential for-, and are dedicated to AM symbiosis, often show a pattern of conservation in all AM-host plants, whereas they are lost in all non-hosts like Arabidopsis (Delaux et al., 2014; Bravo et al., 2016). Since the evolution of VAMP721d/e largely follows this pattern, and since knockdown of these VAMPs does not affect root development (Ivanov et al., 2012), we conclude that they are dedicated to symbiosis. I will refer to these SNAREs that are dedicated to symbiosis as 'symbiotic' SNAREs while I will call their paralogs 'non-symbiotic', even though the latter ones may be involved in symbiosis as well. The existence of symbiotic VAMPs provided the starting point for the research presented in this thesis.

\section{Do (hemi-)biotrophic pathogens require VAMP721d/e to form haustoria?}

Besides mutualist symbionts, also (hemi-)biotrophic filamentous pathogens infect living plant cells in which they form haustoria that are surrounded by a peri-haustorial membrane. The peri-haustorial membrane forms a pathogenic host-microbe interface. To delineate the trafficking regulated by the symbiotic VAMPs, we first questioned whether also haustorium formation depends on VAMP721d and -e. To form haustoria, the pathogen must somehow force the plant into producing the peri-haustorial membrane by making use of pre-existing plant pathways. It has been a long-standing hypothesis that pathogens depend on plant mechanisms involved in symbiotic host-microbe interface formation to forma a pathogenic host-microbe interface (Parniske, 2000; Rey \& Schornack, 2013). In Chapter 2, I have shown that the hemi-biotrophic oomycete Phytophthora palmivora is still able to form haustoria in the roots of Medicago mutants in symbiotic signalling genes that are required for the formation of a symbiotic host-microbe interface. This is in line 
with similar observation by Rey and colleagues (Rey et al., 2015). Further, I have shown that also VAMP721d/e are not required for haustorium formation: In root cultures where VAMP721d/e are knocked down to levels where arbuscule formation is blocked, haustoria are still formed by Phytophthora palmivora. This shows that the formation of a pathogenic host-microbe interface is regulated independent of symbiotic signalling, and does not depend on the same adaptations to exocytosis as symbiotic host-microbe interface formation. In line with this, it was shown by several independent labs, that the peri-haustorial membrane of several different pathogens accumulates endosome markers Rab5, and sometimes Rab7, suggesting that other trafficking pathways than exocytosis are involved in their biogenesis (Lu et al., 2012; Bozkurt et al., 2015; Inada et al., 2016).

\section{The expansion of regulators of exocytosis related to symbiosis}

It is a major question in cell biology how plants target vesicles in a polarized fashion to create distinguishable plasma membrane subdomains such as the host-microbe interface. One simple way to achieve this is the use of multiple secretory pathways; different populations of vesicles to target different cargo to the different domains. The requirement for dedicated VAMPs for the formation of a host-microbe interface raised the idea that they may mark an additional exocytosis pathway. We hypothesized that the duplication and neo-/ subfuctionalization of symbiotic VAMPs alone cannot be sufficient to define a new pathway, since at least components that distinguish between different membrane domains are required. Therefore, we analysed whether the duplication of VAMPs is part of a more general expansion of regulators of the exocytosis machinery, and whether this expansion resulted in a new exocytosis pathway.

In Chapter 3, we focussed in particular on target membrane SNAREs (t-SNAREs). T-SNAREs were promising candidates to have symbiosis dedicated family members, since secretory t-SNAREs are encoded by small gene families, interact with v-SNAREs, and may therefore determine the location of VAMP721d/e mediated vesicle fusion. We found that only the Qa-type t-SNARE SYP132 follows the conservation pattern of symbiosis dedicated genes (Delaux et al., 2013b); it is conserved in all AM-hosts, while it is lost in all AM non-hosts. We found that in dicot plants, SYP132 is spliced into two splice-forms that we named SYP132a and SYP132 $\beta$. In non-symbiotic root tissues, SYP132 $\beta$ is the dominant splice-form, whereas in nodules and arbuscular cells, splicing shifts towards SYP132a. RNAi of SYP132a blocks the full development of arbuscules; some fine branches are formed, but the arbuscules collapse pre-maturely. During rhizobium symbiosis, RNAi of SYP132a inhibits the release and differentiation of symbiosomes. We found that a knockout mutation of the total SYP132 gene was lethal and RNAi of SYP132 $\beta$ did not result in transgenic roots. This shows that SYP132 $\beta$ likely is essential for root development. Similar results were obtained by Pan and co-workers (Pan et al., 2016). Finally, by comparing the sequences of SYP132a and $-\beta$ to that of the SYP132 of monocots, we could show that SYP132a is the most ancient splice-form, while SYP132 $\beta$ evolved later. Together, this shows that SYP132 $\alpha$ is a symbiosis dedicated t-SNARE. 
In addition to v- and t-SNAREs, also other key regulators of exocytosis were found to be duplicated in relation to symbiosis. Before fusion, vesicles are docked to their target membrane by a tethering complex. Different trafficking pathways make use of different tethering complexes. In the case of exocytosis, this is the exocyst complex (Zhang et al., 2010b). The tethering of vesicles is the first step in vesicle fusion, and may therefore be of major importance for the targeting of vesicles to their right location. The exocyst consists of eight subunits, of which in particular the EXO70 subunit has been duplicated many times, to be encoded by a large gene family. It was found that one EXO7O family member, EXO70l, is specifically required for arbuscule formation. (Zhang et al., 2015b). The EXO70 subunit associates with the target membrane and is able to determine the exocyst complex localization, and may as such determine the location of vesicle fusion. Different EXO70 subunits are involved in different biological processes involving localized secretion (Sekereš et al., 2017). Since multiple EXO70 subunits can be expressed in one cell, including arbuscular cells (Harrison \& Ivanov, 2017), it has been suggested that different paralogs can regulate the traffic to different plasma membrane domains to create plasma domains of distinct protein composition (Žárský \& Potocký, 2010; Harrison \& Ivanov, 2017; Sekereš et al., 2017). EXO70l was shown to interact with VAPYRIN (VPY). Both EXO70I and VPY were shown to be transcriptionally induced by the AM symbiosis signalling transcription factor RAM1 (Park et al., 2015; Pimprikar et al., 2016; Chapter 1), linking the common symbiosis signalling pathway to the regulation of traffic to the peri-arbuscular membrane (Zhang et al., 2015b). Mutation of EXO70l does not affect host-microbe interface formation during rhizobium symbiosis (Zhang et al., 2015b). This implies that other EXO70 homologs enable traffic to the symbiosomes.

Besides the regulators of exocytosis that were empirically shown to be dedicated to symbiosis, other regulators were found based on the conservation of AM symbiosis dedicated genes in AM hosts and loss in non-host species. A number of studies have exploited this pattern on a whole genome scale to identify new symbiosis dedicated genes (Delaux et al., 2014; Favre et al., 2014; Bravo et al., 2016). In these studies, also SYP132 and EXO70l were retrieved. In addition, symbiosis related duplication of the genes encoding SEC14 and MLO were found. These proteins were shown to be involved in membrane phospholipid composition and micro domain formation. Although their exact role is unknown, mutations in these proteins affects secretory traffic (Bhat et al., 2005; Panstruga, 2005; Curwin et al., 2009).

The symbiosis associated duplication of different proteins involved in exocytosis raises the question whether these duplications are independent, each allowing the optimization of traffic during symbiosis in its own way, or whether the different symbiosis specific proteins act together. The most extreme case of the latter is that simultaneous duplication of multiple genes encoding regulators of exocytosis resulted in the emergence of a new exocytosis pathway to separate traffic to the host-microbe interface from traffic to 
the plasma membrane. One approach to determine whether the different proteins act together is to determine when they evolved. In Chapter 3, I have shown that SYP132a evolved at the base of the angiosperms, and is conserved between monocots and dicots. A similar conservation pattern was found for EXO70I (Zhang et al., 2015b; Bravo et al., 2016). However, in Chapter 4 I have shown that monocots and the basal dicot Aquilegia do not have a VAMP721d/e ortholog. Therefore, the symbiosis dedicated VAMPs most likely have evolved at the base of the dicots, after the spit of Aquilegia. This means that the selective advantage of having a symbiosis dedicated t-SNARE or EXO7O ortholog was in the common angiosperm ancestor independent of the presence of a symbiosis dedicated v-SNARE, which is still the case in monocots. Nevertheless, the conservation of symbiosis dedicated VAMPs may still be related to the presence of other symbiosis dedicated regulators of exocytosis.

The next question that should be addressed following the identification of symbiosis dedicated regulators of vesicle traffic, is whether these can be sufficient to form a trafficking pathway that can be separated from default traffic to the plasma membrane. The different well established trafficking pathways towards the Golgi, ER, vacuole and plasma membrane are regulated by different v-SNAREs, t-SNAREs, tethering complexes, coat proteins, and small GTPases. Comparison of the protein sequences encoding these proteins shows that the proteins performing each step in the different trafficking pathways are related, and the result of an ancient duplication in the last common eukaryotic ancestor (Cavalier-Smith, 2002; Dacks \& Doolittle, 2002; Jékely, 2003; Yoshizawa et al., 2006; Dacks $\&$ Field, 2007). Thus, by duplication of all of these regulators, new trafficking pathways were created. If a more recent evolution of an additional secretory pathway depends on duplication of the same regulators, then a novel small GTPase and coat protein are still not discovered. Using a modelling approach, it was shown that the minimal components to create distinguishable membrane compartments are a set of v-SNAREs and t-SNAREs that selectively interact with each other, as well as proteins that allow cargo selection into vesicles such as coat proteins (Heinrich \& Rapoport, 2005). This would also suggest that a protein that allows cargo selection into vesicles is still missing from the list of symbiosis dedicated regulators if they encode a separate trafficking pathway. A possible explanation for this is that the parameters in the phylogenomics studies were chosen to select for genes that are conserved between monocots and dicots. If a symbiosis dedicated pathway evolved in dicots specifically, regulators like VAMP721d/e are not picked up. Alternatively, the duplication of regulators of exocytosis does not represent the duplication of secretory pathways. 


\section{Are there multiple secretory pathways that allow the separation of vesicle traffic to the host-microbe interface and the plasma membrane?}

To understand the degree to which vesicle traffic to the host-microbe interface formation is regulated differently from exocytosis towards the plasma membrane, I compared the localization and SNARE interactions of symbiotic SNAREs and their non-symbiotic paralogs in Chapter 4. Further, I tested whether the non-symbiotic SNAREs are able to replace their symbiotic paralogs when expressed at the right level by complementation of the symbiotic SNARE RNAi. These experiments showed clearly that there is no selectivity in SNARE interactions and that the expression of the symbiotic SNAREs is the main determinant for their requirement for arbuscule formation, and not any specific adaptation on protein level. This shows that the symbiosis dedicated SNAREs do not mark a separate exocytosis pathway.

The conclusion that the symbiosis dedicated SNAREs do not mark a symbiosis dedicated trafficking pathway does not mean that such a pathway does not exist. The comparison of other symbiosis dedicated key regulators like EXO7OI and other proteins identified by phylogenomics to their non-symbiotic paralogs may provide new clues on the regulation of membrane trafficking during host-microbe interface formation. This may again point to a dedicated secretory pathway. However, without SNAREs specifically involved in a separate trafficking pathway, the alternative model to target proteins specifically to the host-microbe interface proposed by Pumplin and co-workers is far more likely (Pumplin et al., 2012). These authors have shown that the timing of expression of a gene encoding a membrane protein is a major determinant for the localization of this protein. For example the phosphate transporter PT1 will be exclusively located on the plasma membrane of arbuscular cells when its expression is driven by the $35 \mathrm{~S}$ promoter that switches of when arbuscule formation starts. However, the same protein will be exclusively present on the peri-arbuscular membrane when its expression is driven by the PT4 promoter that switches on when arbuscule formation starts (Pumplin et al., 2012). Based on their findings, the authors conclude that a default traffic to the plasma membrane is transiently redirected to the peri-arbuscular membrane. It is interesting to note that the switch of splicing of SYP132 from predominantly SYP132 $\beta$ in cortical cells to SYP132a in arbuscular cells may coincide with the switch of secretion from the plasma membrane to the peri-arbuscular membrane. This would mean that the two spliceforms of SYP132 do mark different membrane domains, even if they are not targeted differently.

Like during AM symbiosis, the use of a single exocytosis pathway for trafficking to both the plasma membrane and the host-microbe interface may apply to symbiosome formation during rhizobium symbiosis as well. In line with this, there has not been a single report showing the exclusive localization of proteins towards the plasma membrane when they are expressed in cells where symbiosomes are present. The transient re-orientation 
of exocytosis implies that during arbuscule development and possibly during the full arbuscule life-time, there is no traffic to the plasma membrane. Such a scenario could explain the short lifetime of arbuscules, but still seems unlikely, as the plasma membrane normally depends on a constant cycling of vesicles and proteins for its maintenance (Jürgens, 2004). In case of rhizobium symbiosis, the cells in which bacteria are released continue growing. Furthermore, plasma membrane proteins expressed from the Leghaemoglobin promoter, which is only active in cells containing symbiosomes, are transported to the plasma membrane as well as the symbiosomes (Limpens et al., 2009). Thus, if also symbiosome formation depends on default exocytosis, the secretory traffic is only partially redirected to the host-microbe interface. It is likely that this is also the case in arbuscular cells, although the relative amount of traffic towards the arbuscule may be much higher resulting in the apparent exclusive localisation of fluorescent markers towards the peri-arbuscular membrane.

To fully understand the regulation of exocytosis during arbuscule formation, all of the identified regulators of exocytosis should be compared to their non-symbiotic paralogs. It would for example be interesting to test the functional specialisation of EXO70I compared to other EXO70 homologs. Although multiple EXO70 paralogs were shown to be expressed in arbuscular cells, EXO70l is the highest expressed family member (Harrison \& Ivanov, 2017), and thus its expression may be the main reason for its requirement during arbuscule formation similar to the symbiotic SNAREs. To find functional differences between paralogs, the trans-complementation of plant mutants is most straightforward and highly informative, as was done for example for the AM-symbiosis dedicated fatty acid biosynthesis enzyme FatM. FatM mutants can be fully complemented by the non-symbiotic FatB gene, when expressed from the FatM promoter, showing that it is required to increase the production of fatty acids through existing biochemical pathways (Bravo et al., 2017). When plant mutants are not available, complementation of RNAi can be used as an alternative strategy, as shown in chapters 3 and 4.

\section{How do SNAREs involved in symbiosis differ from their non-symbiotic paralogs?}

Although the complementation of RNAi was a useful approach to show that the symbiotic SNAREs do not encode a separate exocytosis pathway, it is unsuitable to study more subtle phenotypes that can be masked by the biological variation caused by RNAi and the use of composite plants. For this, the syp132a-1 mutant described in chapter 5 is more suitable. In this mutant, the a-specific exon including the splicing acceptor site has been deleted, resulting in a constitutive splicing of SYP132 transcripts into SYP132 $\beta$. The amount of arbuscules formed in this mutant, and the morphology of these arbuscules is indistinguishable from wild type plants, which is in line with the idea SYP132 $\beta$ mediates the fusion of the same vesicles as SYP132a. Nevertheless, the biomass of syp132a-1 mutants remains significantly lower compared to wild type, when the plants are mycorrhized. This 
suggests that there is a functional difference between SYP132 $\alpha$ and $-\beta$, that is not related to arbuscule formation, but which affects arbuscule functionality.

To understand how symbiotic SNAREs can be functionally different, while they mediate the fusion of the same vesicles, it is useful to consider the current knowledge on other SNAREs that are specialized for different processes. The evolutionary stability of 7 orthogroups of SYP1 and 4 orthogroups of VAMP72 proteins in dicots (Chapter 4) suggests that each of these orthogroups is essential for maximal fitness of these plants. These SNAREs differ greatly in their level and pattern of expression in different plant tissues (Enami et al., 2009), and mutations in some of these SNAREs have phenotypes associated with specific biological processes (Lauber et al., 1997; Collins et al., 2003). In particular the different proteins encoded by the Qa-type t-SNARE family have different properties that are potentially important for their role in different biological processes. Nevertheless, there is still a high level of functional redundancy between family members. The classic use of the term redundancy refers to genetic redundancy, where knock out mutations in a gene do not lead to a phenotype (full redundancy), or a phenotype that is less severe than would be expected based on the activity of that gene (partial redundancy). Here, I use the term functional redundancy to describe the ability of proteins to perform the same biochemical action in a cell, if expressed at the same level. To illustrate how SNAREs can be adapted to specific processes or redundant compared to their paralogs, I will first review the current knowledge on specialisation and redundancy of plant SNAREs in different biological processes. This is by no means a complete overview of all plant SNARE research, but a selection of the best studied systems that provide useful inspiration on adaptation of symbiotic SNAREs to host-microbe interface formation. To allow comparison of genes between plant species, I will use roman numbering to refer to conserved orthogroups instead of genes as introduced in Chapters 3 \& 4 . I will use the prefix At, Mt, Nt and Hv to refer to individual genes of Arabidopsis thaliana, Medicago truncatula, Nicotiana tabacum and Hordeum vulgare respectively.

\section{VAMP72VII: A new vacuolar trafficking route}

Within the VAMP72 family, VAMP72VII has diverged from the other VAMP72 homologs at the base of the seed plants (Chapter 4). It has neofunctionalized to be involved in vesicle trafficking to either the vacuole or the plasma membrane. AtVAMP727 mediates vesicle fusion to the tonoplast, where it interacts with the vacuolar Qa-SNARE SYP22 (Uemura et al., 2004; Ebine et al., 2011). On the tonoplast, it is required for the transport of proteins to protein storage vacuoles (Ebine et al., 2008). In addition to vacuolar SNAREs, it can also interact with plasma membrane SNARE AtSYP121 (Ebine et al., 2011). In Chapter 4, I have shown that the interaction with plasma membrane SNAREs is not limited to MtSYP121, but extends to MtSYP131, MtSYP132a and MtSYP132 $\beta$. Further, I have shown that also Medicago VAMP727 accumulates on the vacuole and punctuate compartments, showing 
a conservation of its role in vacuolar traffic across different plant species. The role of the VAMP72VII orthogroup in vacuolar vesicle traffic functionally separates it from the other VAMP72 homologs, that do not mediate traffic to the vacuole, and which accumulate on the plasma membrane when overexpressed (Uemura et al., 2004).

\section{Cytokinesis}

During plant cytokinesis, a cell plate or phragmoplast is formed that divides the two daughter cells. The formation of a cell plate is a special case of exocytosis, involving homotypic vesicle fusion; the fusion of vesicles with each other instead of with a planar target membrane. This requires the presence of both $\mathrm{v}$ - and t-SNAREs on the same vesicle. The Arabidopsis SYP11/ ortholog; AtSYP111 (also known as KNOLLE), is specialized for the formation of a cell plate during cell division. Atsyp111 mutants form many incomplete cell plates leading to strongly disturbed patterns of cell division. The plants remain severely dwarfed and are nonviable (Lukowitz et al., 1996). The turnover rate of AtSYP111 is high compared to other Qa SNAREs like AtSYP121 and AtSYP132, and its expression is strictly limited to cells in anaphase (Lauber et al., 1997; Reichardt et al., 2011). This effectively confines the AtSYP111 protein to the phragmoplast. AtSYP111 can form multiple redundant SNARE complexes; It can form a trimeric SNARE complex with Qb+Qc-SNARE AtSNAP33 and AtVAMP721, or a tetrameric complex with Qb-SNARE AtNPSN11, Qc-SNARE AtSYP71 and AtVAMP721 (El Kasmi et al., 2013). The AtSYP111 protein is specialized to be active only at the plane of cell division. The linker sequence between the helical domain and the SNARE domain is bound by the regulatory protein SEC11/KEULE. This binding results in the stabilisation of the open conformation of AtSYP111. SEC11 is transported to the plane of cell division independent of AtSYP111, strictly confining AtSYP111 activity to the right location (Park et al., 2012). The AtSYP111 domain essential for SEC11 binding in open conformation is unique to SYP111, thus its evolution represents a specific adaptation of a SNARE to a process. Despite its unique properties, the macroscopic phenotype Arabidopsis syp111 mutants can still be complemented by AtSYP112 (Müller et al., 2003), and AtSYP132 (Reichardt et al., 2011), when expressed from the AtSYP111 promoter. This shows that there is a high level of functional redundancy between SNAREs when just the ability to form a new cell wall is considered. There may still be a difference between the different syntaxins in the amount of protein that is required to form this cell wall (Reichardt et al., 2011), suggesting that the more accurate spatial regulation of AtSYP111 results in a more efficient cell wall formation.

\section{Penetration resistance}

Plants can defend themselves against invading powdery mildew fungi by the local delivery of callose containing cell wall reinforcements called papillae at the site of attempted penetration by the fungus (Nielsen et al., 2012). In mutant screens for plants 
compromised in this ability in barley and Arabidopsis, SYP12l orthologs AtSYP121/PEN1 and HvSYP121/ROR2 were identified (Collins et al., 2003).The formation of papillae is delayed in Atsyp 121 mutants (Assaad et al., 2004). Arabidopsis has two SYP12l orthologs; AtSYP121 and AtSYP122 that are the result of a Brasicaceae specific duplication. Despite their relatively recent split, the two proteins are functionally different. The penetration resistance phenotype of Atsyp 121 mutants cannot be complemented by overexpression of AtSYP122 (Nielsen et al., 2012). Also expression of AtSYP132 does not restore penetration resistance in Atsyp121 (Reichardt et al., 2011). The functional specialisation of AtSYP121 is not related to the SNARE complexes it forms; It mediates the fusion of AtVAMP721/722 labelled vesicles (Kwon et al., 2008). These VAMPs also form complexes with AtSYP122 and AtSYP132 (Pajonk et al., 2008; Yun et al., 2013; Chapter 4). Instead of the complexes it forms, the functional specialisation of AtSYP121 is related to its dynamic behaviour. whereas AtSYP122 and AtSYP132 are stable on the plasma membrane, AtSYP121 is continuously cycling between the plasma membrane and endosomes. This results in accumulation of AtSYP121 in so called BFA-bodies after treatment with the fungal toxin Brefeldin A (BFA). BFA inhibits GNOM that is involved in budding of vesicles from endosomes and the plasma membrane, which is required for cycling of proteins between these compartments. In contrast to AtSYP121, AtSYP122 and AtSYP132 remain on the plasma membrane upon BFA treatment (Reichardt et al., 2011; Nielsen \& Thordal-Christensen, 2012). The recycling of AtSYP121 is crucial for its role in penetration resistance as mutations in GNOM or BFA treatment block callose appositions and penetration resistance. AtSYP121 accumulates within the papillae were it is located on exosomes (Meyer et al., 2009). These exosomes are the result of fusion of multi-vesicular bodies tot the papillae (An et al., 2006). As the SNARE domain of AtSYP121 is on the inside of the exosomes, it cannot have a relevant role in vesicle fusion anymore at this location (Nielsen \& Thordal-Christensen, 2013). Therefore, it has been suggested that AtSYP121 functions on endosomes instead of the plasma membrane during penetration resistance (Nielsen et al., 2012).

\section{$\mathrm{K}^{+}$-uptake/voltage dependent secretion}

A screen for tobacco proteins involved in ABA-mediated stomatal closure identified the tobacco SYP12l ortholog NtSYP121/SYR1 (Leyman et al., 1999). Following this, it was shown that Arabidopsis AtSYP121 interacts directly with $\mathrm{K}^{+}$-Channels KAT1 and KC1, which shifts their voltage sensitivity, increasing $\mathrm{K}^{+}$-uptake (Honsbein et al., 2009). This interaction depends on the FxRF motif at its N-terminus of AtSYP121 (Grefen et al., 2010), that binds to the voltage sensing domain of the $\mathrm{K}^{+}$-Channels (Grefen et al., 2015). The AtSYP121 FxRF motif is lost in AtSYP122, but highly conserved among SYP12l orthologs in dicots with each species having at least one SYP12I carrying the FXRF motif. The coupling of AtSYP121 mediated exocytosis and $\mathrm{K}^{+}$-uptake was hypothesized to be important to balance the growth of plant cells with their solute content to maintain turgor pressure (Grefen \& Blatt, 
2008). Besides increasing $\mathrm{K}^{+}$-uptake, the interaction between AtSYP121 and $\mathrm{K}^{+}$-channels also increases exocytosis in regions with a membrane potential that opens the $\mathrm{K}^{+}$-channels (Grefen et al., 2015). This is most likely the case because the interaction of AtSYP121 with $\mathrm{K}^{+}$-channels stabilizes the open conformation of the syntaxin. This offers alternative/ additional explanations for the interaction between $\mathrm{K}^{+}$-channels and SYP12I, namely that it makes SYP12I mediated traffic voltage dependent. This means that membrane potential can provide a spatial cue for secretion. Further, it provides a negative feedback loop, since secretion decreases the membrane potential by opening of $\mathrm{K}^{+}$-channels, which in turn inhibits secretion.

\section{Root hair growth}

The Arabidopsis SYP12IV ortholog AtSYP123 was found to be highly expressed in trichoblasts and localizes to the tip of growing root hairs (Enami et al., 2009) The polar localisation of AtSYP123 depends on its recycling, as it accumulates in BFA bodies after BFA treatment (Ichikawa et al., 2014). Mutation of Atsyp123 results in only a mild reduction of root hair length. This is because AtSYP123 is partially redundant with SYP13/ ortholog AtSYP132 that is also expressed in trichoblasts. Knockdown of AtSYP132 by amiRNAs reduces root hair length, showing that also AtSYP132 functions in root hair growth, even though it does not localize specifically to the tip (Ichikawa et al., 2014). Based on the requirement of both AtSYP123 and AtSYP132 for full root hair growth, it was postulated that the two SNAREs must act in concert during root hair growth. Interestingly, AtSYP123 is not widely conserved between plant species. It does not have an own conserved orthogroup, but is embedded within the SYP12IV group that seems in other plant species to be mainly involved in pollen tube growth (see below). For example both Medicago SYP12IV orthologs MtSYP124 and MtSYP125 are not expressed in roots, but only in flowers, like the remaining two Arabidopsis SYP12IV orthologs AtSYP124 and AtSYP125. This means that either the role of AtSYP123 in root hair growth can be taken over completely by SYP13/ in other species, or that its role is fulfilled by other SNAREs. For this, SYP12I orthologs are likely candidates, since they are in Medicago also highly expressed in trichoblasts. Like AtSYP123, Medicago SYP12I proteins also cycle between the plasma membrane and endosomes as they accumulate in BFA compartments (not shown), and AtSYP121 accumulates at the tip of Arabidopsis root hairs when expressed in these cells (Ichikawa et al., 2014). It is therefore likely that AtSYP123 is functionally fully redundant to Medicago SYP12I proteins during root hair growth.

\section{Pollen tube growth}

Pollen tube growth is highly similar to root hair growth as it is also a tip-growing system, that depends on both SYP12IV and SYP13/ orthologs that are partially redundant (Slane et al., 2017). In Arabidopsis these are AtSYP124 and AtSYP125, which accumulate 
slightly behind the growing apex (Ângelo Silva et al., 2010; Ul-Rehman et al., 2011) and AtSYP131 that uniformly labels the plasma membrane of pollen tubes (Ichikawa et al., 2015). Knockout mutations in either of these three syntaxins does not result in an obvious phenotype. However, double mutation of AtSYP124 and AtSYP125 do not segregate in a Mendelian ratio, as these mutations were ineffectively transferred through the male line. Triple mutants in AtSYP124, AtSYP125 and AtSYP131 were never recovered as these were unable to be transmitted through the male line (Slane et al., 2017). This suggest that AtSYP124, AtSYP125 and AtSYP131 are redundantly required for pollen tube growth. This shows, that even though SYP12IV orthologs are functionally adapted to accumulate at the growing tip of pollen tubes, the relevance of this adaptation is limited, as pollen tubes are still growing in syp124/syp125 double mutants due to AtSYP131 mediated secretion.

In general, the mechanisms of functional specialization of SNAREs in the processes described above does not involve specific interactions between pairs of $v$ - and t-SNAREs. Because of this, most SNAREs can be replaced by non-related paralogs if these are expressed in the same cell. However, the redundancy between SNAREs is sometimes partial. Even small and seemingly trivial differences in root hair length, pollen tube growth rate or accuracy of cell plate formation may be the driving force for the evolution and conservation of specialized SNAREs. The same principle may be true for host-microbe interface formation.

\section{Symbiosis dedicated SNAREs}

Below, I discuss possible scenarios that can explain the relevance of symbiosis dedicated SNAREs. More than one of these scenarios may be true, since the specialization of the symbiotic VAMPs may have a different reason than the specialization of SYP13lla. This is in line with the different time points of their evolution. Whereas VAMP72II evolved in dicots, SYP13lla evolved at the base of the angiosperms. Thus, the advantage of SYP13II in monocots is independent of the presence of symbiotic VAMPs. Finally, the role of symbiotic SNAREs in AM symbiosis is sufficient for their conservation. Therefore, it is possible that the functional specialization of symbiotic SNARE proteins is not as relevant for rhizobium symbiosis as for AM symbiosis.

symbiotic SNAREs are required for their high expression in cells were an host-microbe interface is formed

The most straightforward explanation for the evolution of symbiosis dedicated SNAREs is their level of expression in cells where a host-microbe interface is formed. Since the host-microbe interface represents a great expansion of the total amount of membrane, it seems likely that its formation requires an upregulation of the exocytosis machinery components, including SNAREs. Therefore, the use of specific SNARE genes for host microbe interface formation can be advantageous even if there is not any specialization 
on the protein level. There are however several reasons that argue against the hypothesis that the need for and increased amount of SNAREs is the sole reason for the evolution of symbiosis dedicated SNAREs: 1). The complementation of MtVAMP721d/e RNAi by MtVAMP721a only partially rescues the mycorrhization level (Chapter 4). The complementation of MtSYP132a knockout by MtSYP132 $\beta$ in the syp132a-1 mutant seems to result in arbuscules of a lower functionality, as the mycorrhized plants stay smaller (Chapter 5). 2). During both rhizobium symbiosis and AM symbiosis, the level of MtVAMP721a is drastically downregulated. This is not in line with the hypothesis that the total level of SNAREs needs to be increased irrespective of which SNARE. Sinharoy and co-workers found that the expression of MtVAMP721a is actively suppressed in nodules by direct binding of the RSD transcription factor to the MtVAMP721a promoter (Sinharoy et al., 2013). Thus, instead of just upregulating the total amount of VAMP72 proteins, MtVAMP721a is purposely down-regulated in nodules. Also the splicing of MtSYP132 results in the replacement of MtSYP132 $\beta$ by MtSYP132a without altering the total transcript levels. Together, these observations strongly suggest that it does matter which SNARE is used for host-microbe interface formation, and that the proteins encoded by symbiotic SNAREs are functionally different from their non-symbiotic paralogs. 3).The symbiotic SNAREs behave different from their paralogs. In Chapter 5, I have shown that the stability of MtSYP132 $a$ and $-\beta$ on the peri-arbuscular membrane are different. Further, the amount of VAMPs that co-purify with MtSYP132a is lower than the other SYPs. Finally, during the co-IP experiments described in Chapter 4, we noted for example that the amount of 3HA-MtVAMP721a in the protein extracts was in general lower than the amount of 3HA-MtVAMP721e, while both constructs were expressed from the PT4 promoter. Thus, the turnover rate of MtVAMP721a in arbuscular cells appears to be higher than that of MtVAMP721e.

\section{Restriction of SNARE activity}

In Chapter 4, I have shown that less VAMPs co-precipitate with MtSYP132a compared to its non-symbiotic paralogs. The lower amount of complexes that we found may be the result of a lower activity of MtSYP132a. This would indicate that SYP132a has a dual role in vesicle fusion to the host- microbe interface; a positive role enabling vesicle fusion that can be taken over by any other plasma membrane syntaxins, as well as a negative role in restricting vesicle fusion. This latter role may be unique to MtSYP132a. The restriction of vesicle fusion may be achieved by control of its open and closed conformation in analogy to the regulation of AtSYP121 and AtSYP111 by interaction with $\mathrm{K}^{+}$-channels or SEC11. A stricter control of MtSYP132a may be important to prevent cis-SNARE complexes before vesicle fusion, which may be important to increase the efficiency of vesicle fusion. An alternative explanation for the lower amount of VAMPs that co-purify with MtSYP132a is that not the formation of SNARE complexes is restricted, but that the cis-SNARE complexes are more rapidly assembled. Both scenarios can result in a lower amount of 
MtSYP132a containing SNARE complexes. After vesicle fusion, VAMPs are endocytosed to be used in a next round of vesicle fusion, or to be degraded. When t-SNAREs are still in complex with these VAMPs, they may be co-endocytosed lowering their stability on the target membrane. The observed increased stability of MtSYP132a on the peri-arbuscular membrane compared to MtSYP132 $\beta$ can therefore be a result of the lower interaction with VAMPs. When also MtVAMP721e is overexpressed, more SYP132a containing complexes may be formed, resulting in the observed faster turnover of MtSYP132a.

The accumulation of MtVAMP721d/e seems to be regulated on protein level. In non-symbiotic cortex cells, these VAMPs could not be observed by immunolocalization. In contrast, in arbuscular cells, in cells where rhizobia are released and in dividing cells where a cell plate is formed the proteins accumulated (Ivanov et al., 2012). Therefore, also the use of MtVAMP721d/e may allow plants to restrict SNARE activity to these cells, by controlling protein turnover.

\section{Increased stability on the peri-arbuscular membrane}

In Chapter3, I have shown that MtSYP132 $\alpha$ and $-\beta$ show a clear difference in protein localization during the partial collapse of arbuscules. Whereas MtSYP132a is relatively more present on the healthy branches, MtSYP132 $\beta$ is relatively more present on the encasement surrounding the collapsed branches. In Chapter 5, we argue that the localisation of MtSYP132 $\alpha$ and $-\beta$ to different domains of degrading arbuscules is mainly the result of a difference in protein stability on the healthy parts of arbuscules, and not a difference in abundance on or in the encasement surrounding the collapsed parts. As argued above, the difference in protein stability may be a direct result of the lower level of interaction with MtVAMP721e. Therefore, the difference in localisation of MtSYP132a and $-\beta$ to healthy and degrading arbuscule parts may be without biological relevance. Alternatively, the prolonged presence of MtSYP132a on the peri-arbuscular membrane is required to sustain exocytosis after arbuscule formation, which may be required for its full functionality.

\section{SNAREs as chaperones for peri-arbuscular membrane proteins}

The expression of membrane proteins in arbuscular cells is a major determinant for their localization on the peri-arbuscular membrane. In addition, proteins should be loaded into vesicles to exit the endoplasmic reticulum (ER) and the Golgi apparatus. In non-symbiotic cortex cells, different proteins are allowed to leave the ER compared to and arbuscular cells. For example the plasma membrane protein AtPIP2 is retained in the ER in arbuscular cells, while the peri-arbuscular membrane specific phosphate transporter MtPT4 is retained in the ER in non-symbiotic cells (Pumplin et al., 2012). These observations are best explained if these proteins rely on chaperones to be exported from the ER. The different expression of their chaperones in symbiotic and non-symbiotic cells may explain their ability to reach 
the plasma membrane or peri-arbuscular membrane. Symbiotic SNAREs may act as or interact with these chaperones to allow some peri-arbuscular membrane specific cargo to exit the ER. This scenario is more likely for MtVAMP721d/e than for MtSYP132a, since MtSYP132a is not required for PT4 to leave the ER (Chapter 5).

\section{Interaction with peri-arbuscular membrane located transporters}

Finally, the specialization of MtSYP132a may be unrelated to membrane trafficking: In analogy with the interaction of AtSYP121 with $\mathrm{K}^{+}$-Channels that increases the $\mathrm{K}^{+}$-uptake by these channels, MtSYP132a may interact with nutrient transporters on the peri-arbuscular membrane. This interaction may be required for the full functionality of these transporters and the peri-arbuscular membrane.

\section{Concluding remarks}

Studies on differences in behaviour of SNARE proteins are essential to understand the mechanisms of functional specialization of symbiotic SNAREs. It is however difficult to judge which of these are biologically most relevant. For example, the increased stability of MtSYP132a on the peri-arbuscular membrane may be essential for its function, or a mere side effect of a lower interaction with VAMPs. To get more insight into the difference between MtSYP132a and other t-SNAREs, a mass spec approach on immunoprecipitated SNAREs could be used to identify differences in protein interactions. If MtSYP132a would be a chaperone for specific host-microbe interface components, or modulates the activity of transporters on the peri-arbuscular membrane, this will be revealed using this approach.

By generating a mutant line in which MtSYP132a transcripts are replaced by MtSYP132 $\beta$ transcripts, we have generated a unique tool to study the biological relevance of the functional specialization of MtSYP132a. The lower biomass of mycorrhized syp132a-1 roots shows that the use of the right t-SNARE is highly important for plan fitness. Therefore, further research should focus on this mutant. Since SYP132a acts at the peri-arbuscular membrane, it is most likely that the reduced biomass of syp132a-1 plants is the result of a reduced functionality of arbuscules or arbuscule cells, via mechanisms as discussed above. To get more insight in the mechanisms that explain how a t-SNARE affects arbuscule morphology, it should first be tested whether this phenomena is completely independent of its role in vesicle traffic. For this, it is useful to analyse the phenotype of the arbuscules in this mutant by transmission electron microscopy to screen for morphological defects of the peri-arbuscular membrane or other membrane compartments that are invisible with light microscopy. This research has the potential to uncover new mechanisms that determine the functionality of arbuscules that is independent from their morphology. 
o

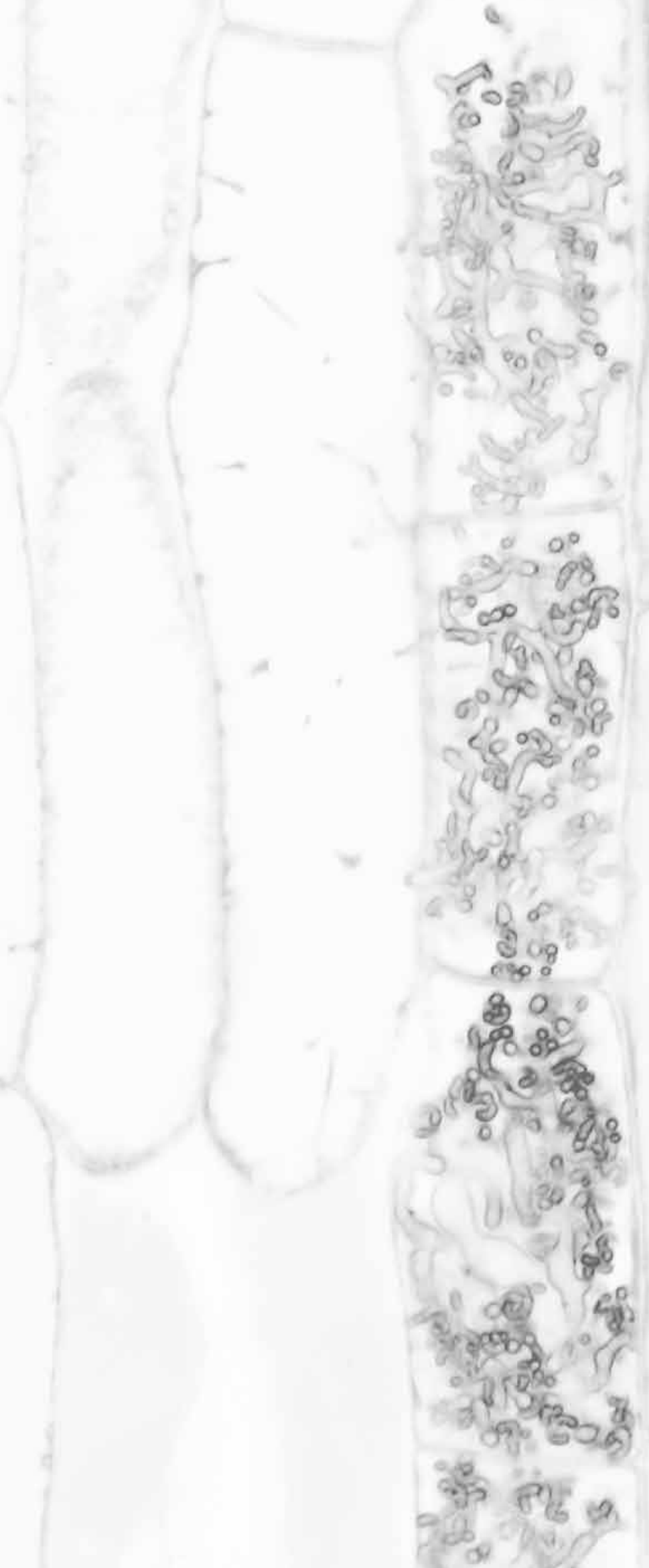


Summary 
At the heart of endosymbiosis microbes are hosted inside living cells in specialized membrane compartments that from a host-microbe interface, where nutrients and signal are efficiently exchanged. Such symbiotic interfaces include arbuscules produced by arbuscular mycorrhiza (AM) and organelle-like symbiosomes formed during the rhizobium-legume symbiosis. Also during pathogenic interactions, microbes such as biotrophic fungi and oomycetes are hosted in specialized membrane compartments called haustoria. The formation of such new membrane compartments requires a major reorganization of the host endomembrane system, with a special role for the targeting of secretory/exocytotic vesicles and their cargo to the newly forming interfaces. In this thesis, I studied how exocytotic membrane traffic is regulated to facilitate the formation and maintenance of a host-microbe interface. Therefore, I especially focussed on the role of SNARE (Soluble NSF Attachment Protein Receptor) proteins, as key components of the exocytotic machinery, in symbiotic interface formation.

In Chapter 1, I introduce the different symbioses in which host-microbe interfaces are formed, and the role of the host-microbe interface in these symbioses. Further, I introduce the evolutionary relationship between the different symbioses: AM symbiosis is the most ancient endosymbiosis in plants, which provided the blueprint for different symbioses that evolved later; other symbiotic microbes including rhizobia co-opted the signalling program and adaptations to membrane trafficking required for arbuscule formation, to be hosted inside cells. Finally, I will introduce the symbiosis dedicated SNAREs as key regulators of exocytosis to form a host-microbe interface.

In Chapter 2, we tested the long-standing hypothesis that pathogens make use of the AM symbiotic program to allow the formation of haustoria. To test this, we set up a pathosystem using the biotrophic oomycete Phytophthora palmivora that is able to form haustoria in Medicago truncatula root cells. Using $M$. truncatula mutants impaired in $\mathrm{AM}$ and rhizobium symbioses, we demonstrated that neither the common symbiotic signalling genes, nor symbiosis dedicated regulators of vesicle trafficking are required for haustorium formation. This showed that biotrophic pathogens like P. palmivora, do not hijack the symbiotic program to be accommodated inside plant cells.

In Chapter 3, we identified the t-SNARE SYP132a as a key regulator of both arbuscule and symbiosome formation. During vesicle fusion, a vesicle SNARE ( $v$-SNARE) on the vesicle forms a complex with a target membrane SNAREs (t-SNAREs) on the target membrane. Previous work in our lab identified specific exocytotic v-SNAREs required for arbuscule and symbiosome formation. We identified the t-SNARE counterpart SYP132, and demonstrated that in most dicot plants SYP132 is spliced into two spliceforms; SYP132a and SYP132 $\beta$. Interestingly, alternative splicing of SYP132 leading to the dominant use of a SYP132a-specific last exon coincides with the accommodation of AM fungi in arbuscule forming root cortex cells and rhizobium bacteria in nodule cells. Using a spliceform-specific RNAi 
construct, we showed that SYP132a is specifically required for the formation of a stable host-microbe interface in both AM symbiosis and rhizobium symbiosis. Furthermore, we showed that during arbuscular collapse, the two spliceforms localize differently to healthy and degrading arbuscule branches. These results indicated that alternative splicing of SYP132 allows plants to replace a t-SNARE involved in traffic to the plasma membrane with a t-SNARE that is more stringent in its localization to functional arbuscules.

The evolutionary expansion of SNAREs in plants has been hypothesized to have allowed the adaptation of exocytosis to different biological processes. In Chapter 4, we studied what makes the symbiotic SNAREs so special in comparison to their non-symbiotic family members, of which many are also expressed in arbuscule cells. We hypothesized that symbiotic SNAREs define a distinct secretory pathway, that ensures specificity of protein delivery to the host-microbe interface. We show that all tested SYP1 family proteins, and most of the non-symbiotic VAMP72 members, were able to complement the defect in arbuscule formation upon knock-down of their symbiotic counterparts when expressed at sufficient levels. This functional redundancy is in line with the ability of all tested vand t-SNARE combinations to form SNARE complexes at the peri-arbuscular membrane. This showed that the symbiotic SNAREs do not selectively interact to define a distinct vesicle trafficking pathway, but that their essential role in arbuscule formation can be largely explained by their dominant expression level. Interestingly, the symbiotic t-SNARE SYP132a appeared to occur less in SNARE complexes with v-SNAREs compared to the non-symbiotic syntaxins in the arbuscule cells, suggesting a more strict regulation of symbiotic SNARE complexes at the interface.

Since the alternative splicing of SYP132 does not affect the total transcript levels, we hypothesized that there must be a functional difference between SYP132 $\alpha$ and $-\beta$, potentially leading to subtle phenotypes that may have gone undetected in the Agrobacterium rhizogenes mediated complementation approach applied in Chapter 4. In Chapter 5, we therefore generated and characterized a stable mutant line in which all SYP132 transcripts are constitutively spliced into the non-symbiotic SYP132 $\beta$ form. Although this mutant is normally colonized by AM fungi, with no effects on arbuscule morphology, it has a severely reduced biomass after mycorrhization. This hints to a yet unknown role for SYP132a to control arbuscule functionality, and offers an explanation for the evolutionary conservation of the SYP132 alternative splicing in dicot plants. Finally, using fluorescent timer fusions to both SYP132 isoforms, we showed that the difference in localization of the two proteins during arbuscular collapse is the result of a different (endocytic) turnover of the two spliceforms at the healthy/functional arbuscule branches, possibly due to a difference in interactions with VAMPs. Together, our data show that, although both SYP132 isoforms can mediate arbuscule formation, SYP132 $\alpha$ is functionally different from SYP132 $\beta$, which may reveal new aspects of the control of nutrient exchange. 
In Chapter 6, I discuss the data generated during my thesis research in relation to additional symbiosis dedicated regulators of exocytosis, as well as in relation to other biological processes that depend on specific secretory SNAREs. Following our conclusion that the symbiotic SNAREs do not mark a separate exocytosis pathway, but are functionally different from non-symbiotic SNAREs, I will speculate on the possible scenarios in which symbiosis dedicated SNAREs are specialized for host-microbe interface functionality. 
References 
Albrecht C, Geurts R, Lapeyrie F, Bisseling T. 1998. Endomycorrhizae and rhizobial nod factors both require SYM8 to induce the expression of the early nodulin genes PsENOD5 and PsENOD12A. The Plant Journal 15: 605-614.

Alexander T, Meier R, Toth R, Weber HC. 1988. Dynamics of arbuscule development and degeneration in mycorrhizas of Triticum aestivum L. and Avena sativa L. with reference to Zea mays L. New Phytologist 110: 363-370.

Alexander T, Toth R, Meier R, Weber HC. 1989. Dynamics of arbuscule development and degeneration in onion, bean, and tomato with reference to vesicular-arbuscular mycorrhizae in grasses. Canadian Journal of Botany 67: 2505-2513.

Allen FHE, Friend J. 1983. Resistance of potato tubers to infection by Phytophthora infestans: a structural study of haustorial encasement. Physiological Plant Pathology 22: 285-IN4.

Amor B Ben, Shaw SL, Oldroyd GED, Maillet F, Penmetsa RV, Cook D, Long SR, Dénarié J, Gough C. 2003. The NFP locus of Medicago truncatula controls an early step of Nod factor signal transduction upstream of a rapid calcium flux and root hair deformation. The Plant Journal 34: 495-506.

An Q, Hückelhoven R, Kogel KH, van Bel AJE. 2006. Multivesicular bodies participate in a cell wall-associated defence response in barley leaves attacked by the pathogenic powdery mildew fungus. Cellular Microbiology 8: 1009-1019.

Ângelo Silva P, UI-Rehman R, Rato C, Di Sansebastiano G-P, Malhó R. 2010. Asymmetric localization of Arabidopsis SYP124 syntaxin at the pollen tube apical and sub-apical zones is involved in tip growth. BMC Plant Biology 10: 179.

Arrighi J-F, Barre A, Amor B Ben, Bersoult A, Soriano LC, Mirabella R, de Carvalho-Niebel F, Journet E-P, Ghérardi M, Huguet T, et al. 2006. The Medicago truncatula Lysin Motif-Receptor-like Kinase Gene Family Includes NFP and New Nodule-Expressed Genes. Plant Physiology 142: 265.

Assaad FF, Qiu JL, Youngs H, Ehrhardt D, Zimmerli L, Kalde M, Wanner G, Peck SC, Edwards H, Ramonell $\mathbf{K}$, et al. 2004. The PEN1 syntaxin defines a novel cellular compartment upon fungal attack and is required for the timely assembly of papillae. Molecular biology of the cell 15: 5118-5129.

Augé RM. 2001. Water relations, drought and vesicular-arbuscular mycorrhizal symbiosis. Mycorrhiza 11: 3-42.

Auriac M-C, Timmers ACJ. 2007. Nodulation studies in the model legume Medicago truncatula: advantages of using the constitutive EF1alpha promoter and limitations in detecting fluorescent reporter proteins in nodule tissues. Molecular plant-microbe interactions: MPMI 20: 1040-1047.

Badreddine I, Lafitte C, Heux L, Skandalis N, Spanou Z, Martinez Y, Esquerre-Tugaye M-T, Bulone V, Dumas B, Bottin A. 2008. Cell Wall Chitosaccharides Are Essential Components and Exposed Patterns of the Phytopathogenic Oomycete Aphanomyces euteiches. Eukaryotic Cell 7: 1980-1993.

Baier MC, Keck M, Gödde V, Niehaus K, Küster H, Hohnjec N. 2010. Knockdown of the symbiotic sucrose synthase MtSucS1 affects arbuscule maturation and maintenance in mycorrhizal roots of Medicago truncatula. Plant Physiology 152: 1000-14.

Bainard LD, Bainard JD, Newmaster SG, Klironomos JN. 2011. Mycorrhizal symbiosis stimulates endoreduplication in angiosperms. Plant, Cell and Environment 34: 1577-1585.

Balestrini R, Bonfante P. 2014. Cell wall remodeling in mycorrhizal symbiosis: a way towards biotrophism. Frontiers in plant science 5: 237.

Baxter L, Tripathy S, Ishaque N, Boot N, Cabral A, Kemen E, Thines M, Ah-Fong A, Anderson R, Badejoko W, et al. 2010. Signatures of adaptation to obligate biotrophy in the Hyaloperonospora arabidopsidis genome. Science 330: 1549-51.

Bécard G, Fortin JA. 1988. Early events of vesicular-arbuscular mycorrhiza formation on Ri T-DNA transformed roots. New Phytologist 108: 211-218.

Becquer A, Trap J, Irshad U, Ali MA, Claude P. 2014. From soil to plant, the journey of P through trophic relationships and ectomycorrhizal association. Frontiers in Plant Science 5: 1-7.

Bhat R a, Miklis M, Schmelzer E, Schulze-Lefert P, Panstruga R. 2005. Recruitment and interaction dynamics of plant penetration resistance components in a plasma membrane microdomain. Proceedings of the $\mathrm{Na}$ tional Academy of Sciences of the United States of America 102: 3135-3140.

Bozkurt TO, Belhaj K, Dagdas YF, Chaparro-Garcia A, Wu CH, Cano LM, Kamoun S. 2015. Rerouting of Plant Late Endocytic Trafficking Toward a Pathogen Interface. Traffic 16: 204-226. 
Bozsokia Z, Cheng J, Feng F, Gysela K, Vinther M, Andersen KR, Oldroyd G, Blaise M, Radutoiu S, Stougaard J. 2017. Receptor mediated chitin perception in legume roots is functionally separable from Nod factor perception Short title: Legume receptors perceiving chitin. Proceedings of the National Academy of Sciences.

Bradbury SM, Peterson RL, Bowley SR. 1991. Interactions between three alfalfa nodulation genotypes and two Glomus species. New Phytologist 119: 115-120.

Bravo A, Brands M, Wewer V, Dörmann P, Harrison MJ. 2017. Arbuscular mycorrhiza-specific enzymes FatM and RAM2 fine-tune lipid biosynthesis to promote development of arbuscular mycorrhiza. New Phytologist 214: $1631-1645$.

Bravo A, York T, Pumplin N, Mueller LA, Harrison MJ. 2016. Genes conserved for arbuscular mycorrhizal symbiosis identified through phylogenomics. Nature Plants 2: doi: 10.1038/nplants.2015.208.

Breuillin-Sessoms F, Floss DS, Gomez SK, Pumplin N, Ding Y, Levesque-Tremblay V, Noar RD, Daniels D a., Bravo A, Eaglesham JB, et al. 2015. Suppression of Arbuscule Degeneration in Medicago truncatula phosphate transporter4 Mutants Is Dependent on the Ammonium Transporter 2 Family Protein AMT2;3. The Plant Cell 27: 1352-1366.

Cai J, Liu X, Vanneste K, Proost S, Tsai W-C, Liu K-W, Chen L-J, He Y, Xu Q, Bian C, et al. 2014. The genome sequence of the orchid Phalaenopsis equestris. Nature Genetics 47: 65-72.

Carotenuto G, Chabaud M, Miyata K, Capozzi M, Takeda N, Kaku H, Shibuya N, Nakagawa T, Barker DG, Genre A. 2017. The rice LysM receptor-like kinase OsCERK1 is required for the perception of short-chain chitin oligomers in arbuscular mycorrhizal signaling. New Phytologist 214: 1440-1446.

Catalano CM, Czymmek KJ, Gann JG, Sherrier DJ. 2007. Medicago truncatula syntaxin SYP132 defines the symbiosome membrane and infection droplet membrane in root nodules. Planta 225: 541-550.

Catalano CM, Lane WS, Sherrier DJ. 2004. Biochemical characterization of symbiosome membrane proteins from Medicago truncatula root nodules. Electrophoresis 25: 519-531.

Catoira R, Galera C, de Billy F, Penmetsa R, Journet E, Maillet F, Rosenberg C, Cook D, Gough C, Dénarié J. 2000. Four Genes of Medicago truncatula Controlling Components of a Nod Factor Transduction Pathway. The Plant Cell 12: 1647-1666.

Cavalier-Smith T. 2002. The phagotrophic origin of eukaryotes and phylogenetic classification of Protozoa Introduction : revising the neomuran theory of the origin of eukaryotic cells. International Journal of Systematic and Evolutionary Microbiology 52: 297-354.

Cerri MR, Frances L, Laloum T, Auriac M-C, Niebel A, Oldroyd GED, Barker DG, Fournier J, de Carvalho-Niebel F. 2012. Medicago truncatula ERN Transcription Factors: Regulatory Interplay with NSP1/NSP2 GRAS Factors and Expression Dynamics throughout Rhizobial Infection. Plant Physiology 160: 2155-2172.

Cerri MR, Wang Q, Stolz P, Folgmann J, Frances L, Katzer K, Li X, Heckmann AB, Wang TL, Downie JA, et al. 2017. The ERN1 transcription factor gene is a target of the CCaMK/CYCLOPS complex and controls rhizobial infection in Lotus japonicus. New Phytologist 215: 323-337.

Chabaud M, De Carvalho-Niebel F, Barker DG. 2003. Efficient transformation of Medicago truncatula cv. Jemalong using the hypervirulent Agrobacterium tumefaciens strain AGL1. Plant Cell Reports 22: 46-51.

Chandran D, Inada N, Hather G, Kleindt CK, Wildermuth MC. 2010. Laser microdissection of Arabidopsis cells at the powdery mildew infection site reveals site-specific processes and regulators. Proceedings of the National Academy of Sciences of the United States of America 107: 460-465.

Chao CCT, Mihic A, Tsushima RG, Gaisano HY. 2011. SNARE protein regulation of cardiac potassium channels and atrial natriuretic factor secretion. Journal of Molecular and Cellular Cardiology 50: 401-407.

Charpentier M, Bredemeier R, Wanner G, Takeda N, Schleiff E, Parniske M. 2008. Lotus japonicus CASTOR and POLLUX are ion channels essential for perinuclear calcium spiking in legume root endosymbiosis. The Plant Cell 20: 3467-79.

Charpentier M, Sun J, Martins TV, Radhakrishnan G V, Findlay K, Soumpourou E, Thouin J, Very AA, Sanders D, Morris RJ, et al. 2016. Nuclear-localized cyclic nucleotide-gated channels mediate symbiotic calcium oscillations. Science 352: 1102-1105.

Clarholm M. 1985. Interactions of bacteria, protozoa and plants leading to mineralization of soil nitrogen. Soil Biology and Biochemistry 17: 181-187. 
Clarke VC, Loughlin PC, Day DA, Smith PMC. 2014. Transport processes of the legume symbiosome membrane. Frontiers in Plant Science 5: 1-9.

Clarke VC, Loughlin PC, Gavrin A, Chen C, Brear EM, Day DA, Smith PMC. 2015. Proteomic Analysis of the Soybean Symbiosome Identifies New Symbiotic Proteins. Molecular \& Cellular Proteomics 14: 1301-1322.

Collins NC, Thordal-Christensen H, Lipka V, Bau S, Kombrink E, Qiu J-LL, Hückelhoven R, Stein M, Freialdenhoven A, Somerville SC, et al. 2003. SNARE-protein-mediated disease resistance at the plant cell wall. Nature 425: 973-977.

Cox G, Sanders F. 1974. Ultrastructure of the Host-Fungus Interface in a Vesicular-Arbuscular Mycorrhiza. New Phytologist 73: 901-912.

Crooks GE, Hon G, Chandonia JM, Brenner SE. 2004. WebLogo: A sequence logo generator. Genome Research 14: $1188-1190$.

Curwin AJ, Fairn GD, McMaster CR. 2009. Phospholipid transfer protein Sec14 is required for trafficking from endosomes and regulates distinct trans-golgi export pathways. Journal of Biological Chemistry 284: 7364-7375.

Dacks JB, Doolittle WF. 2002. Novel syntaxin gene sequences from Giardia, Trypanosoma and algae: implications for the ancient evolution of the eukaryotic endomembrane system. Journal of cell science 115: 1635-42.

Dacks JB, Field MC. 2007. Evolution of the eukaryotic membrane-trafficking system: origin, tempo and mode. Journal of cell science 120: 2977-85.

Delaux PM, Bécard G, Combier JP. 2013a. NSP1 is a component of the Myc signaling pathway. New Phytologist 199: 59-65.

Delaux PM, Séjalon-Delmas N, Bécard G, Ané JM. 2013b. Evolution of the plant-microbe symbiotic 'toolkit'. Trends in Plant Science 18: 298-304.

Delaux PM, Varala K, Edger PP, Coruzzi GM, Pires JC, Ané JM. 2014. Comparative Phylogenomics Uncovers the Impact of Symbiotic Associations on Host Genome Evolution. PLoS Genetics 10.

Doyle JJ. 2011. Phylogenetic Perspectives on the Origins of Nodulation. Molecular Plant-Microbe Interactions 24: 1289-1295.

Dubuke ML, Maniatis S, Shaffer SA, Munson M. 2015. The exocyst subunit Sec6 interacts with assembled exocytic SNARE complexes. Journal of Biological Chemistry 290: 28245-28256.

Duplessis S. 2011. Obligate biotrophy features unraveled by the genomic analysis of rust fungi. Proceedings of the National Academy of Sciences of the United States of America: 1-23.

Ebine K, Fujimoto M, Okatani Y, Nishiyama T, Goh T, Ito E, Dainobu T, Nishitani A, Uemura T, Sato MH, et al. 2011. A membrane trafficking pathway regulated by the plant-specific RAB GTPase ARA6. Nature cell biology 13: 853-859.

Ebine K, Okatani Y, Uemura T, Goh T, Shoda K, Niihama M, Morita MT, Spitzer C, Otegui MS, Nakano A, et al. 2008. A SNARE complex unique to seed plants is required for protein storage vacuole biogenesis and seed development of Arabidopsis thaliana. The Plant Cell 20: 3006-3021.

Enami K, Ichikawa M, Uemura T, Kutsuna N, Hasezawa S, Nakagawa T, Nakano A, Sato MH. 2009. Differential expression control and polarized distribution of plasma membrane-resident SYP1 SNAREs in Arabidopsis thaliana. Plant and Cell Physiology 50: 280-289.

Endre G, Kereszt A, Kevei Z, Mihacea S, Kaló P, Kiss GB. 2002. A receptor kinase gene regulating symbiotic nodule development. Nature 417: 962-966.

Favre P, Bapaume L, Bossolini E, Delorenzi M, Falquet L, Reinhardt D. 2014. A novel bioinformatics pipeline to discover genes related to arbuscular mycorrhizal symbiosis based on their evolutionary conservation pattern among higher plants. BMC Plant Biology 14: 333.

Floss DS, Gomez SK, Park HJ, MacLean AM, Müller LM, Bhattarai KK, Lévesque-Tremblay V, Maldonado-Mendoza IE, Harrison MJ. 2017. A Transcriptional Program for Arbuscule Degeneration during AM Symbiosis Is Regulated by MYB1. Current Biology 27: 1206-1212.

Floss DS, Levy JG, Lévesque-Tremblay V, Pumplin N, Harrison MJ. 2013. DELLA proteins regulate arbuscule formation in arbuscular mycorrhizal symbiosis. Proceedings of the National Academy of Sciences of the United States of America 110: E5025-34. 
Fonouni-Farde C, Tan S, Baudin M, Brault M, Wen J, Mysore KS, Niebel A, Frugier F, Diet A. 2016. DELLA-mediated gibberellin signalling regulates Nod factor signalling and rhizobial infection. Nature Communications 7: 12636.

Garcia K, Delaux PM, Cope KR, Ané JM. 2015. Molecular signals required for the establishment and maintenance of ectomycorrhizal symbioses. New Phytologist 208: 79-87.

Gavrin A, Kaiser BN, Geiger D, Tyerman SD, Wen Z, Bisseling T, Fedorova EE. 2014. Adjustment of Host Cells for Accommodation of Symbiotic Bacteria: Vacuole Defunctionalization, HOPS Suppression, and TIP1g Retargeting in Medicago. The Plant Cell 26: 3809-22.

Geldner N, Anders N, Wolters H, Keicher J, Kornberger W, Muller P, Delbarre A, Ueda T, Nakano A, Jürgens G. 2003. The Arabidopsis GNOM ARF-GEF mediates endosomal recycling, auxin transport, and auxin-dependent plant growth. Cell 112: 219-230.

Genre A, Chabaud M, Balzergue C, Puech-Pagès V, Novero M, Rey T, Fournier J, Rochange S, Bécard G, Bonfante $P$, et al. 2013. Short-chain chitin oligomers from arbuscular mycorrhizal fungi trigger nuclear $\mathrm{Ca} 2+$ spiking in Medicago truncatula roots and their production is enhanced by strigolactone. New Phytologist 198: 190-202.

Genre A, Chabaud M, Timmers T, Bonfante P, Barker DG. 2005. Arbuscular mycorrhizal fungi elicit a novel intracellular apparatus in Medicago truncatula root epidermal cells before infection. The Plant Cell 17: 3489-3499.

Genre A, Ivanov S, Fendrych M, Faccio A, Žárský V, Bisseling T, Bonfante P. 2012. Multiple exocytotic markers accumulate at the sites of perifungal membrane biogenesis in arbuscular mycorrhizas. Plant and Cell Physiology 53: 244-255.

Genre A, Ortu G, Bertoldo C, Martino E, Bonfante P. 2009. Biotic and Abiotic Stimulation of Root Epidermal Cells Reveals Common and Specific Responses to Arbuscular Mycorrhizal Fungi. Plant Physiology 149: 1424-1434.

Genre A, Russo G. 2016. Does a Common Pathway Transduce Symbiotic Signals in Plant-Microbe Interactions? Frontiers in plant science 7.

Gherbi H, Markmann K, Svistoonoff S, Estevan J, Autran D, Giczey G, Auguy F, Peret B, Laplaze L, Franche C, et al. 2008. SymRK defines a common genetic basis for plant root endosymbioses with arbuscular mycorrhiza fungi, rhizobia, and Frankiabacteria. Proceedings of the National Academy of Sciences of the United States of America 105: 4928-4932.

Giraldo MC, Dagdas YF, Gupta YK, Mentlak TA, Yi M, Martinez-Rocha AL, Saitoh H, Terauchi R, Talbot NJ, Valent B. 2013. Two distinct secretion systems facilitate tissue invasion by the rice blast fungus Magnaporthe oryzae. Nature Communications 4: 1-12.

Gobbato E, Marsh JF, Vernié T, Wang E, Maillet F, Kim J, Miller JB, Sun J, Bano SA, Ratet P, et al. 2012. A GRAS-type transcription factor with a specific function in mycorrhizal signaling. Current Biology 22: 22362241.

Gobbato E, Wang E, Higgins G, Bano SA, Henry C, Schultze M, Oldroyd GED. 2013. RAM1 and RAM2 function and expression during arbuscular mycorrhizal symbiosis and Aphanomyces euteiches colonization. Plant signaling \& behavior 8: $1-5$.

Grefen C, Blatt MR. 2008. SNAREs-molecular governors in signalling and development. Current Opinion in Plant Biology 11:600-609.

Grefen C, Chen Z, Honsbein A, Donald N, Hills A, Blatt MR. 2010. A Novel Motif Essential for SNARE Interaction with the K+ Channel KC1 and Channel Gating in Arabidopsis. The Plant Cell 22: 3076-3092.

Grefen C, Karnik R, Larson E, Lefoulon C, Wang Y, Waghmare S, Zhang B, Hills A, Blatt MR. 2015. A vesicle-trafficking protein commandeers Kv channel voltage sensors for voltage-dependent secretion. Nature Plants 1: 15108.

Guether M, Neuhauser B, Balestrini R, Dynowski M, Ludewig U, Bonfante P. 2009. A Mycorrhizal-Specific Ammonium Transporter from Lotus japonicus Acquires Nitrogen Released by Arbuscular Mycorrhizal Fungi. Plant Physiology 150: 73-83.

Güimil S, Chang H-S, Zhu T, Sesma A, Osbourn A, Roux C, loannidis V, Oakeley EJ, Docquier M, Descombes $\mathbf{P}$, et al. 2005. Comparative transcriptomics of rice reveals an ancient pattern of response to microbial colonization. Proceedings of the National Academy of Sciences of the United States of America 102: 8066-70. 
Gutjahr C, Banba M, Croset V, An K, Miyao A, An G, Hirochika H, Imaizumi-Anraku H, Paszkowski U. 2008. Arbuscular Mycorrhiza-Specific Signaling in Rice Transcends the Common Symbiosis Signaling Pathway. The Plant Cell 20: 2989-3005.

Gutjahr C, Parniske M. 2013. Cell and developmental biology of arbuscular mycorrhiza symbiosis. Annual Review of Cell and Developmental Biology 29: 593-617.

Gutjahr C, Radovanovic D, Geoffroy J, Zhang Q, Siegler H, Chiapello M, Casieri L, An K, An G, Guiderdoni E, et al. 2012. The half-size ABC transporters STR1 and STR2 are indispensable for mycorrhizal arbuscule formation in rice. The Plant Journal 69: 906-920.

Hardham AR. 2007. Cell biology of plant-oomycete interactions. Cellular Microbiology 9: 31-39.

Harpole WS, Ngai JT, Cleland EE, Seabloom EW, Borer ET, Bracken MES, Elser JJ, Gruner DS, Hillebrand H, Shurin JB, et al. 2011. Nutrient co-limitation of primary producer communities. Ecology Letters 14: 852-862.

Harrison MJ. 2012. Cellular programs for arbuscular mycorrhizal symbiosis. Current Opinion in Plant Biology 15: 691-698.

Harrison MJ, Dewbre GR, Liu JY. 2002. A phosphate transporter from Medicago truncatula involved in the acquisiton of phosphate released by arbuscular mycorrhizal fungi. The Plant Cell 14: 2413-2429.

Harrison MJ, Ivanov S. 2017. Exocytosis for endosymbiosis: membrane trafficking pathways for development of symbiotic membrane compartments. Current Opinion in Plant Biology 38: 101-108.

Heinrich R, Rapoport TA. 2005. Generation of nonidentical compartments in vesicular transport systems. Journal of Cell Biology 168: 271-280.

Hirsch AM, Bang M, Ausubel FM. 1983. Ultrastructural Analysis of Ineffective Alfalfa Nodules Formed By nif::Tn5 Mutants of Rhizobium meliloti. Journal of Bacteriology 155: 367-380.

Hirsch S, Kim J, Munoz A, Heckmann AB, Downie JA, Oldroyd GED. 2009. GRAS Proteins Form a DNA Binding Complex to Induce Gene Expression during Nodulation Signaling in Medicago truncatula. The Plant Cell 21: 545-557.

Hogekamp C, Arndt D, Pereira P a., Becker JD, Hohnjec N, Kuster H, Küster H, Kuster H. 2011. Laser Microdissection Unravels Cell-Type-Specific Transcription in Arbuscular Mycorrhizal Roots, Including CAAT-Box Transcription Factor Gene Expression Correlating with Fungal Contact and Spread. Plant Physiology 157: 2023-2043.

Hohnjec N, Lenz F, Fehlberg V, Vieweg MF, Baier MC, Hause B, Küster H. 2009. The signal peptide of the Medicago truncatula modular nodulin MtNOD25 operates as an address label for the specific targeting of proteins to nitrogen-fixing symbiosomes. Molecular plant-microbe interactions : MPMI 22: 63-72.

Honsbein A, Blatt MR, Grefen C. 2011. A molecular framework for coupling cellular volume and osmotic solute transport control. Journal of Experimental Botany 62: 2363-2370.

Honsbein A, Sokolovski S, Grefen C, Campanoni P, Pratelli R, Paneque M, Chen Z, Johansson I, Blatt MR. 2009. A Tripartite SNARE-K+ Channel Complex Mediates in Channel-Dependent $K+$ Nutrition in Arabidopsis. The Plant Cell 21: 2859-2877.

Horváth B, Yeun LH, Domonkos Á, Halász G, Gobbato E, Ayaydin F, Miró K, Hirsch S, Sun J, Tadege M, et al. 2011. Medicago truncatula IPD3 Is a Member of the Common Symbiotic Signaling Pathway Required for Rhizobial and Mycorrhizal Symbioses. Molecular Plant-Microbe Interactions 24: 1345-1358.

Huisman R, Hontelez J, Mysore KS, Wen J, Bisseling T, Limpens E. 2016. A symbiosis-dedicated SYNTAXIN OF PLANTS 13ll isoform controls the formation of a stable host-microbe interface in symbiosis. New Phytologist 211: 1338-1351.

Huisman R, Ovchinnikova E, Bisseling T, Limpens E. 2012. Endocytic Accomodation of Microbes in Plants. Šamaj, Jozef (ed.), Endocytosis in Plants: 271-295.

Ichikawa M, Hirano T, Enami K, Fuselier T, Kato N, Kwon C, Voigt B, Schulze-Lefert P, Baluška F, Sato MH. 2014. Syntaxin of plant proteins SYP 123 and SYP1 32 mediate root hair tip growth in arabidopsis thaliana. Plant and Cell Physiology 55: 790-800.

Ichikawa M, Iwano M, Sato MH. 2015. Nuclear membrane localization during pollen development and apex-focused polarity establishment of SYP124/125 during pollen germination in Arabidopsis thaliana. Plant Reproduction 28: 143-151. 
Inada N, Betsuyaku S, Shimada TL, Ebine K, Ito E, Kutsuna N, Hasezawa S, Takano Y, Fukuda H, Nakano A, et al. 2016. Modulation of plant RAB GTPase-mediated membrane trafficking pathway at the interface between plants and obligate biotrophic pathogens. Plant and Cell Physiology 57: 1854-1864.

Ivanov S, Fedorova EE, Limpens E, De Mita S, Genre a., Bonfante P, Bisseling T. 2012. Rhizobium-legume symbiosis shares an exocytotic pathway required for arbuscule formation. Proceedings of the National Academy of Sciences 109: 8316-8321.

Ivanov S, Harrison MJ. 2014. A set of fluorescent protein-based markers expressed from constitutive and arbuscular mycorrhiza-inducible promoters to label organelles, membranes and cytoskeletal elements in Medicago truncatula. The Plant Journal 80: 1151-1163.

Javot H, Penmetsa RV, Terzaghi N, Cook DR, Harrison MJ. 2007. A Medicago truncatula phosphate transporter indispensable for the arbuscular mycorrhizal symbiosis. Proceedings of the National Academy of Sciences of the United States of America 104: 1720-1725.

Jékely G. 2003. Small GTPases and the evolution of the eukaryotic cell. BioEssays 25: 1129-1138.

Jiang Y, Wang W, Xie Q, Liu N, Wang D, Zhang X, Yang C, Chen X, Tang D, Wang E. 2017. Plants transfer lipids to sustain colonization by mutualistic mycorrhizal and parasitic fungi. Science 356: 1172-1175.

Jiang W, Zhou H, Bi H, Fromm M, Yang B, Weeks DP. 2013. Demonstration of CRISPR/Cas9/sgRNA-mediated targeted gene modification in Arabidopsis, tobacco, sorghum and rice. Nucleic Acids Research 41.

Jin Y, Liu H, Luo D, Yu N, Dong W, Wang C, Zhang X, Dai H, Yang J, Wang E. 2016. DELLA proteins are common components of symbiotic rhizobial and mycorrhizal signalling pathways. Nature Communications $\mathbf{7}$ : 12433.

Jürgens G. 2004. Membrane Trafficking in Plants. Annual Review of Cell and Developmental Biology 20: $481-504$.

Kalo P, Gleason C, Edwards A, Marsh J, Mitra RM, Hirsch S, Jakab J, Sims S, Long SR, Rogers J, et al. 2005. Nodulation signaling in legumes requires NSP2, a member of the GRAS family of transcriptional regulators. Science 308: 1786-1789.

Kanazawa T, Ueda T. 2017. Exocytic trafficking pathways in plants: why and how they are redirected. New Phytologist 215: 952-957.

Kankanala P, Czymmek K, Valent B. 2007. Roles for rice membrane dynamics and plasmodesmata during biotrophic invasion by the blast fungus. The Plant Cell 19: 706-724.

KarnahI M, Park M, Mayer U, Hiller U, Jürgens G. 2017. ER assembly of SNARE complexes mediating formation of partitioning membrane in Arabidopsis cytokinesis. eLife 6: 1-10.

Karnik R, Grefen C, Bayne R, Honsbein A, Köhler T, Kioumourtzoglou D, Williams M, Bryant NJ, Blatt MR. 2013. Arabidopsis Sec1/Munc18 protein SEC11 Is a competitive and dynamic modulator of SNARE binding and SYP121-dependent vesicle traffic. The Plant Cell 25: 1368-1382.

Karnik R, Zhang B, Waghmare S, Aderhold C, Grefen C, Blatt MR. 2015. Binding of SEC11 Indicates Its Role in SNARE Recycling after Vesicle Fusion and Identifies Two Pathways for Vesicular Traffic to the Plasma Membrane. The Plant Cell 27: 675-694.

El Kasmi F, Krause C, Hiller U, Stierhof Y-D, Mayer U, Conner L, Kong L, Reichardt I, Sanderfoot A a, Jürgens G. 2013. SNARE complexes of different composition jointly mediate membrane fusion in Arabidopsis cytokinesis. Molecular biology of the cell 24: 1593-601.

Kevei Z, Lougnon G, Mergaert P, Horvath G V, Kereszt A, Jayaraman D, Zaman N, Marcel F, Regulski K, Kiss GB, et al. 2007. 3-hydroxy-3-methylglutaryl coenzyme A reductase1 interacts with NORK and is crucial for nodulation in Medicago truncatula. The Plant Cell 19: 3974-3989.

Keymer A, Pimprikar P, Wewer V, Huber C, Brands M, Bucerius SL, Delaux P-M, KlingI V, Von Roepenack-Lahaye E, Wang TL, et al. 2017. Lipid transfer from plants to arbuscular mycorrhiza fungi. elife 0: 1-33.

Kiers ET, Duhamel M, Beesetty Y, Mensah JA, Franken O, Verbruggen E, Fellbaum CR, Kowalchuk GA, Hart MM, Bago A, et al. 2011. Reciprocal Rewards Stabilize Cooperation in the Mycorrhizal Symbiosis. Science 333: 880-882.

Kim H, O'Connell R, Maekawa-Yoshikawa M, Uemura T, Neumann U, Schulze-Lefert P. 2014. The powdery mildew resistance protein RPW8.2 is carried on VAMP721/722 vesicles to the extrahaustorial membrane of haustorial complexes. The Plant Journal 79: 835-847. 
Kistner C, Winzer T, Pitzschke A, Mulder L, Sato S, Kaneko T, Tabata S, Sandal N, Stougaard J, Webb KJ, et al. 2005. Seven Lotus japonicus genes required for transcriptional reprogramming of the root during fungal and bacterial symbiosis. The Plant Cell 17: 2217-2229.

Kleemann J, Rincon-Rivera LJ, Takahara H, Neumann U, van Themaat EVL, van der Does HC, Hacquard S, Stüber K, Will I, Schmalenbach W, et al. 2012. Sequential delivery of host-induced virulence effectors by appressoria and intracellular hyphae of the phytopathogen colletotrichum higginsianum. PLoS Pathogens 8.

Kleine-Vehn J, Dhonukshe P, Sauer M, Brewer PB, Wiśniewska J, Paciorek T, Benková E, Friml J. 2008. ARF GEF-Dependent Transcytosis and Polar Delivery of PIN Auxin Carriers in Arabidopsis. Current Biology 18: 526-531.

Knauf GM, Welter K, Müller M, Mendgen K. 1989. The haustorial host-parasite interface in rust-infected bean leaves after high-pressure freezing. Physiological and Molecular Plant Pathology 34: 519-530.

Kobae Y, Fujiwara T. 2014. Earliest colonization events of rhizophagus irregularis in rice roots occur preferentially in previously uncolonized cells. Plant and Cell Physiology 55: 1497-1510.

Kobae Y, Gutjahr C, Paszkowski U, Kojima T, Fujiwara T, Hata S. 2014. Lipid droplets of arbuscular mycorrhizal fungi emerge in concert with arbuscule collapse. Plant and Cell Physiology 55: 1945-1953.

Kobae Y, Hata S. 2010. Dynamics of periarbuscular membranes visualized with a fluorescent phosphate transporter in arbuscular mycorrhizal roots of rice. Plant and Cell Physiology 51: 341-353.

Kobae Y, Ohmori Y, Saito C, Yano K, Ohtomo R, Fujiwara T. 2016. Phosphate treatment strongly inhibits new arbuscule development but not the maintenance of arbuscule in mycorrhizal rice roots. Plant Physiology 171: pp.00127.2016.

Kobae Y, Tamura Y, Takai S, Banba M, Hata S. 2010. Localized expression of arbuscular mycorrhiza-inducible ammonium transporters in soybean. Plant and Cell Physiology 51: 1411-1415.

Kouchi H, Imaizumi-Anraku H, Hayashi M, Hakoyama T, Nakagawa T, Umehara Y, Suganuma N, Kawaguchi M. 2010. How many peas in a pod? Legume genes responsible for mutualistic symbioses underground. Plant and Cell Physiology 51: 1381-1397.

Krajinski F, Courty P-E, Sieh D, Franken P, Zhang H, Bucher M, Gerlach N, Kryvoruchko I, Zoeller D, Udvardi $\mathbf{M}$, et al. 2014. The H+-ATPase HA1 of Medicago truncatula Is Essential for Phosphate Transport and Plant Growth during Arbuscular Mycorrhizal Symbiosis. The Plant Cell 26: 1808-1817.

Kreitzer G, Schmoranzer J, Low SH, Li X, Gan Y, Weimbs T, Simon SM, Rodriguez-Boulan E. 2003. Three-dimensional analysis of post-Golgi carrier exocytosis in epithelial cells. Nature Cell Biology 5: 126-136.

Krusell L, Krause K, Ott T, Desbrosses G, Krämer U, Sato S, Nakamura Y, Tabata S, James EK, Sandal N, et al. 2005. The sulfate transporter SST1 is crucial for symbiotic nitrogen fixation in Lotus japonicus root nodules. The Plant Cell 17: 1625-1636.

Kwaaitaal M, Keinath NF, Pajonk S, Biskup C, Panstruga R. 2010. Combined bimolecular fluorescence complementation and Forster resonance energy transfer reveals ternary SNARE complex formation in living plant cells. Plant Physiology 152: 1135-1147.

Kwon C, Neu C, Pajonk S, Yun HS, Lipka U, Humphry M, Bau S, Straus M, Kwaaitaal M, Rampelt H, et al. 2008. Co-option of a default secretory pathway for plant immune responses. Nature 451: 835-40.

Łangowski L, Wabnik K, Li H, Vanneste S, Naramoto S, Tanaka H, Friml J. 2016. Cellular mechanisms for cargo delivery and polarity maintenance at different polar domains in plant cells. Cell Discov 2: 16018.

Lauber MH, Waizenegger I, Steinmann T, Schwarz H, Mayer U, Hwang I, Lukowitz W, Jürgens G. 1997. The Arabidopsis KNOLLE protein is a cytokinesis-specific syntaxin. Journal of Cell Biology 139: 1485-1493.

Leigh J, Hodge A, Fitter AH. 2009. Arbuscular mycorrhizal fungi can transfer substantial amounts of nitrogen to their host plant from organic material. New Phytologist 181: 199-207.

Levy J, Bres C, Geurts R, Chalhoub B, Kulikova O, Duc G, Journet E-P, Ane J-M, Lauber E, Bisseling T, et al. 2004. A Putative $\mathrm{Ca} 2+$ and Calmodulin-Dependent Protein Kinase Required for Bacterial and Fungal Symbioses. Science 303: 1361-1364.

Lewis DG, Quirk JP. 1967. Phosphate diffusion in soil and uptake by plants. Plant and Soil 27: 445-453.

Leyman B, Geelen D, Quintero FJ, Blatt MR. 1999. A Tobacco Syntaxin with a Role in Hormonal Control of Guard Cell Ion Channels. Science 283: 537-540. 
Liang Y, Tóth K, Cao Y, Tanaka K, Espinoza C, Stacey G. 2014. Lipochitooligosaccharide recognition: an ancient story. New Phytologist 204: 289-296.

Li R, Rodriguez-Furlan C, Wang J, van de Ven W, Gao T, Raikhel N V, Hicks GR. 2017. Different endomembrane trafficking pathways establish apical and basal polarities. The Plant Cell 29: 90-108.

Limpens E, Franken C, Smit P, Willemse J, Bisseling T, Geurts R. 2003. LysM Domain Receptor Kinases Regulating Rhizobial Nod Factor-Induced Infection. Science 302: 630-633.

Limpens E, Ivanov S, van Esse W, Voets G, Fedorova EE, Bisseling T. 2009. Medicago N2-Fixing Symbiosomes Acquire the Endocytic Identity Marker Rab7 but Delay the Acquisition of Vacuolar Identity. The Plant Cell 21: tpc.108.064410.

Limpens E, Mirabella R, Fedorova E, Franken C, Franssen H, Bisseling T, Geurts R. 2005. Formation of organelle-like N2-fixing symbiosomes in legume root nodules is controlled by DMI2. Proceedings of the National Academy of Sciences of the United States of America 102: 10375-80.

Limpens E, Moling S, Hooiveld G, Pereira PA, Bisseling T, Becker JD, K??ster H. 2013. Cell- and Tissue-Specific Transcriptome Analyses of Medicago truncatula Root Nodules. PLoS ONE 8.

Limpens E, Ramos J, Franken C, Raz V, Compaan B, Franssen H, Bisseling T, Geurts R. 2004. RNA interference in Agrobacterium rhizogenes-transformed roots of Arabidopsis and Medicago truncatula. Journal of Experimental Botany 55: 983-992.

Lipka V, Kwon C, Panstruga R. 2007. SNARE-ware: the role of SNARE-domain proteins in plant biology. Annual review of cell and developmental biology 23: 147-174.

Livak KJ, Schmittgen TD. 2001. Analysis of relative gene expression data using real-time quantitative PCR and. Methods 25: 402-408.

Lu YJ, Schornack S, Spallek T, Geldner N, Chory J, Schellmann S, Schumacher K, Kamoun S, Robatzek S. 2012. Patterns of plant subcellular responses to successful oomycete infections reveal differences in host cell reprogramming and endocytic trafficking. Cellular Microbiology 14: 682-697.

LuginbuehI LH, Menard GN, Kurup S, Van Erp H, Radhakrishnan G V., Breakspear A, Oldroyd GED, Eastmond PJ. 2017. Fatty acids in arbuscular mycorrhizal fungi are synthesized by the host plant. Science 356: 1175-1178.

Lukowitz W, Mayer U, Jürgens G. 1996. Cytokinesis in the Arabidopsis embryo involves the syntaxin-related KNOLLE gene product. Cell 84: 61-71.

Madsen EB, Madsen LH, Radutoiu S, Olbryt M, Rakwalska M, Szczyglowski K, Sato S, Kaneko T, Tabata S, Sandal N, et al. 2003. A receptor kinase gene of the LysM type is involved in legume perception of rhizobial signals. Nature 425: 637-640.

Maillet F, Poinsot V, André O, Puech-Pagès V, Haouy A, Gueunier M, Cromer L, Giraudet D, Formey D, Niebel A, et al. 2011. Fungal lipochitooligosaccharide symbiotic signals in arbuscular mycorrhiza. Nature 469: 58-63.

Marcel S, Sawers R, Oakeley E, Angliker H, Paszkowski U. 2010. Tissue-adapted invasion strategies of the rice blast fungus Magnaporthe oryzae. The Plant Cell 22: 3177-3187.

Marsh JF, Rakocevic A, Mitra RM, Brocard L, Sun J, Eschstruth A, Long SR, Schultze M, Ratet P, Oldroyd GED. 2007. Medicago truncatula NIN Is Essential for Rhizobial-Independent Nodule Organogenesis Induced by Autoactive Calcium/Calmodulin-Dependent Protein Kinase. Plant Physiology 144: 324-335.

Martin F, Kohler A, Murat C, Veneault-Fourrey C, Hibbett DS. 2016. Unearthing the roots of ectomycorrhizal symbioses. Nature Reviews Microbiology 14: 760-773.

Martinez-Arca S, Rudge R, Vacca M, Raposo G, Camonis J, Proux-Gillardeaux V, Daviet L, Formstecher E, Hamburger A, Filippini F, et al. 2003. A dual mechanism controlling the localization and function of exocytic v-SNAREs. Proceedings of the National Academy of Sciences of the United States of America 100: 9011-6.

Matter K, Mellman I. 1994. Mechanisms of cell polarity: Sorting and transport in epithelial cells. Current Opinion in Cell Biology 6: 545-554.

Mcnew JA, Parlati F, Fukuda R, Johnston RJ, Paz K, Paumet F, Sollner TH, Rothman JE. 2000. Compartmental speci city of cellular membrane fusion encoded in SNARE proteins. Nature 407: 153-159.

Meijer HJG, van de Vondervoort PJI, Yin QY, de Koster CG, Klis FM, Govers F, de Groot PWJ. 2006. Identification of cell wall-associated proteins from Phytophthora ramorum. Molecular Plant-Microbe Interactions 19: 1348-1358. 
Mellersh D, Parniske M. 2006. Common symbiosis genes of Lotus japonicus are not required for intracellular accomodation of the rust fungus Uromyces loti. New Phytologist 170: 641-644.

Mellman I, Nelson W. 2008. Coordinated protein sorting, targeting and distribution in polarized cells. Nat Rev Mol Cell Biol 9: 833-845.

Meyer D, Pajonk S, Micali C, O'Connell R, Schulze-Lefert P. 2009. Extracellular transport and integration of plant secretory proteins into pathogen-induced cell wall compartments. The Plant Journal 57: 986-999.

Micali CO, Neumann U, Grunewald D, Panstruga R, O\&apos;Connell R. 2011. Biogenesis of a specialized plant-fungal interface during host cell internalization of Golovinomyces orontii haustoria. Cellular Microbiology 13: 210-226.

Michaelevski I, Chikvashvili D, Tsuk S, Singer-Lahat D, Kang Y, Linial M, Gaisano HY, Fili O, Lotan I. 2003. Direct interaction of target SNAREs with the Kv2.1 channel: Modal regulation of channel activation and inactivation gating. Journal of Biological Chemistry 278: 34320-34330.

Middleton PH, Jakab J, Penmetsa R V., Starker CG, Doll J, Kalo P, Prabhu R, Marsh JF, Mitra RM, Kereszt A, et al. 2007. An ERF Transcription Factor in Medicago truncatula That Is Essential for Nod Factor Signal Transduction. The Plant Cell 19: 1221-1234.

Miller JB, Pratap A, Miyahara A, Zhou L, Bornemann S, Morris RJ, Oldroyd GED. 2013. Calcium/Calmodulin-dependent protein kinase is negatively and positively regulated by calcium, providing a mechanism for decoding calcium responses during symbiosis signaling. The Plant Cell 25: 5053-66.

Mirabella R, Franken C, Krogt GNM, Bisseling T, Geurts R. 2004. Use of the Fluorescent Timer DsRED-E5 as Reporter to Monitor Dynamics of Gene Activity in Plants. Plant Physiology 135: 1879-1887.

De Mita S, Streng A, Bisseling T, Geurts R. 2014. Evolution of a symbiotic receptor through gene duplications in the legume - rhizobium mutualism. New Phytologist: 961-972.

Miyata K, Kozaki T, Kouzai Y, Ozawa K, Ishii K, Asamizu E, Okabe Y, Umehara Y, Miyamoto A, Kobae Y, et al. 2014. The bifunctional plant receptor, OsCERK1, regulates both chitin-triggered immunity and arbuscular mycorrhizal symbiosis in rice. Plant and Cell Physiology 55: 1864-1872.

Moore MJ, Soltis PS, Bell CD, Burleigh JG, Soltis DE. 2010. Phylogenetic analysis of 83 plastid genes further resolves the early diversification of eudicots. Proceedings of the National Academy of Sciences of the United States of America 107: 4623-8.

Morandi D, Prado E, Sagan M, Duc G. 2005. Characterisation of new symbiotic Medicago truncatula (Gaertn.) mutants, and phenotypic or genotypic complementary information on previously described mutants. Mycorrhiza 15: 283-289.

Mosse B. 1973. Advances in the study of vesicular-arbuscular mycorrhiza. Annual revieuw of Phytopathology 3570: 171-196.

Mostov K, Su T, ter Beest M. 2003. Polarized epithelial membrane traffic: conservation and plasticity. Nature cell biology 5: 287-293.

Müller I, Wagner W, Völker A, Schellmann S, Nacry P, Küttner F, Schwarz-Sommer Z, Mayer U, Jürgens G. 2003. Syntaxin specificity of cytokinesis in Arabidopsis. Nature cell biology 5: 531-4.

Murray JD, Muni RRD, Torres-Jerez I, Tang Y, Allen S, Andriankaja M, Li G, Laxmi A, Cheng X, Wen J, et al. 2011. Vapyrin, a gene essential for intracellular progression of arbuscular mycorrhizal symbiosis, is also essential for infection by rhizobia in the nodule symbiosis of Medicago truncatula. The Plant Journal 65 : 244-252.

Nakagawa T, Kaku H, Shimoda Y, Sugiyama A, Shimamura M, Takanashi K, Yazaki K, Aoki T, Shibuya N, Kouchi H. 2011. From defense to symbiosis: Limited alterations in the kinase domain of LysM receptor-like kinases are crucial for evolution of legume-Rhizobium symbiosis. The Plant Journal 65: 169-180.

Nanjareddy K, Arthikala MK, Gómez BM, Blanco L, Lara M. 2017. Differentially expressed genes in mycorrhized and nodulated roots of common bean are associated with defense, cell wall architecture, $\mathrm{N}$ metabolism, and P metabolism. PLOS ONE 12: 1-25.

Nars A, Lafitte C, Chabaud M, Drouillard S, Mélida H, Danoun S, Le Costaouëc T, Rey T, Benedetti J, Bulone V, et al. 2013. Aphanomyces euteiches Cell Wall Fractions Containing Novel Glucan-Chitosaccharides Induce Defense Genes and Nuclear Calcium Oscillations in the Plant Host Medicago truncatula. PLoS ONE 8: 1-13. 
Nielsen ME, Feechan A, Böhlenius H, Ueda T, Thordal-Christensen H. 2012. Arabidopsis ARF-GTP exchange factor, GNOM, mediates transport required for innate immunity and focal accumulation of syntaxin PEN1. Proceedings of the National Academy of Sciences of the United States of America 109: 11443-8.

Nielsen ME, Thordal-Christensen H. 2012. Recycling of Arabidopsis plasma membrane PEN1 syntaxin. Plant signaling \& behavior 7: 1541-1543.

Nielsen ME, Thordal-Christensen H. 2013. Transcytosis shuts the door for an unwanted guest. Trends in Plant Science 18: 611-616.

Novozamsky I, Houba VJG, Eck R van, Vark W van. 1983. A novel digestion technique for multi-element plant analysis. Communications in Soil Science and Plant Analysis 14: 239-248.

O'Connell RJ, Panstruga R. 2006. Tete a tete inside a plant cell: establishing compatibility between plants and biotrophic fungi and oomycetes. New Phytologist 171: 699-718.

O'Connell RJ, Uronu AB, Waksman G, Nash C, Keon JPR, Bailey JA. 1993. Hemibiotrophic infection of Pisum sativum by Colletotrichum truncatum. Plant Pathology 42: 774-783.

Oldroyd GED. 2013. Speak, friend, and enter: signalling systems that promote beneficial symbiotic associations in plants. Nature reviews. Microbiology 11: 252-63.

Oldroyd GED, Long SR. 2003. Identification and Characterization of Nodulation-Signaling Pathway 2 , a Gene of Medicago truncatula Involved in Nod Factor Signaling. Plant Physiology 131: 1027-1032.

Op den Camp R, Streng A, De Mita S, Cao Q, Polone E, Liu W, Ammiraju JSS, Kudrna D, Wing R, Untergasser A, et al. 2011. LysM-Type Mycorrhizal Receptor Recruited for Rhizobium Symbiosis in Nonlegume Parasponia. Science 331: 909-912.

Ovchinnikova E, Journet E-P, Chabaud M, Cosson V, Ratet P, Duc G, Fedorova E, Liu W, den Camp RO, Zhukov V, et al. 2011. IPD3 Controls the Formation of Nitrogen-Fixing Symbiosomes in Pea and Medicago Spp. Molecular Plant-Microbe Interactions 24: 1333-1344.

Pajonk S, Kwon C, Clemens N, Panstruga R, Schulze-Lefert P. 2008. Activity determinants and functional specialization of Arabidopsis PEN1 syntaxin in innate immunity. Journal of Biological Chemistry 283: 2697426984.

Pan H, Oztas O, Zhang X, Wu X, Stonoha C, Wang E, Wang B, Wang D. 2016. A symbiotic SNARE protein generated by alternative termination of transcription. Nature Plants 2: 15197.

Panstruga R. 2005. Serpentine plant MLO proteins as entry portals for powdery mildew fungi. Biochemical Society transactions 33: 389-392.

Park H-J, Floss DS, Levesque-Tremblay V, Bravo A, Harrison MJ. 2015. Hyphal Branching during Arbuscule Development Requires Reduced Arbuscular Mycorrhiza1. Plant Physiology 169: 2774-88.

Park M, Touihri S, Müller I, Mayer U, Jürgens G. 2012. Sec1/Munc18 Protein Stabilizes Fusion-Competent Syntaxin for Membrane Fusion in Arabidopsis Cytokinesis. Developmental Cell 22: 989-1000.

Parniske M. 2000. Intracellular accommodation of microbes by plants: A common developmental program for symbiosis and disease? Current Opinion in Plant Biology 3: 320-328.

Parniske M. 2008. Arbuscular mycorrhiza: the mother of plant root endosymbioses. Nature reviews. Microbiology 6: 763-75.

Petre B, Kamoun S. 2014. How Do Filamentous Pathogens Deliver Effector Proteins into Plant Cells? PLoS Biology 12.

Pierre O, Engler G, Hopkins J, Brau F, Boncompagni E, Hérouart D. 2013. Peribacteroid space acidification: A marker of mature bacteroid functioning in Medicago truncatula nodules. Plant, Cell and Environment 36 2059-2070.

Pimprikar P, Carbonnel S, Paries M, Katzer K, Klingl V, Bohmer MJ, Karl L, Floss DS, Harrison MJ, Parniske $\mathbf{M}$, et al. 2016. A CCAMK-CYCLOPS-DELLA complex activates transcription of RAM1 to regulate arbuscule branching. Current Biology 26: 987-998.

Pozo MJ, Jung SC, Lopez-Raez JA, Azcon-Aguilar C. 2010. Impact of Arbuscular Mycorrhizal Symbiosis on Plant Response to Biotic Stress: The Role of Plant Defense Mechanisms. In: Arbuscular Mycorrhizas: Physiology and Function. 193-207.

Pumplin N, Harrison MJ. 2009. Live-cell imaging reveals periarbuscular membrane domains and organelle location in Medicago truncatula roots during arbuscular mycorrhizal symbiosis. Plant Physiology 151: 809-19. 
Pumplin N, Mondo SJ, Topp S, Starker CG, Gantt JS, Harrison MJ. 2010. Medicago truncatula Vapyrin is a novel protein required for arbuscular mycorrhizal symbiosis. The Plant journal 61: 482-94.

Pumplin N, Zhang X, Noar RD, Harrison MJ. 2012. Polar localization of a symbiosis-specific phosphate transporter is mediated by a transient reorientation of secretion. Proceedings of the National Academy of Sciences of the United States of America 109: E665-72.

Radutoiu S, Madsen LH, Madsen EB, Felle HH, Umehara Y, Grønlund M, Sato S, Nakamura Y, Tabata S, Sandal N, et al. 2003. Plant recognition of symbiotic bacteria requires two LysM receptor-like kinases. Nature 425: 585-592.

Redecker D, Kodner R, Graham LE. 2000. Glomalean fungi from the Ordovician. Science 289: 1920-1921.

Rehman RU, Stigliano E, Lycett GW, Sticher L, Sbano F, Faraco M, Dalessandro G, Di Sansebastiano G Pietro. 2008. Tomato Rab1 1a characterization evidenced a difference between SYP121-dependent and SYP122-dependent exocytosis. Plant and Cell Physiology 49: 751-766.

Reichardt I, Slane D, El Kasmi F, Knöll C, Fuchs R, Mayer U, Lipka V, Jürgens G. 2011. Mechanisms of Functional Specificity Among Plasma-Membrane Syntaxins in Arabidopsis. Traffic 12: 1269-1280.

Rey T, Chatterjee A, Buttay M, Toulotte J, Schornack S. 2015. Medicago truncatula symbiosis mutants affected in the interaction with a biotrophic root pathogen. New Phytologist 206: 497-500.

Rey T, Nars A, Bonhomme M, Bottin A, Huguet S, Balzergue S, Jardinaud MF, Bono JJ, Cullimore J, Dumas B, et al. 2013. NFP, a LysM protein controlling Nod factor perception, also intervenes in Medicago truncatula resistance to pathogens. New Phytologist 198: 875-886.

Rey T, Schornack S. 2013. Interactions of beneficial and detrimental root-colonizing filamentous microbes with plant hosts. Genome biology 14: 121.

Robertson JG, Lyttleton P. 1984. Division of peribacteroid membranes in root nodules of white clover. Journal of cell science 69: 147-57.

Roth LE, Stacey G. 1989. Bacterium release into host-cells of nitrogen-fixing soybean nodules - The symbiosome membrane comes from 3 sources. European Journal of Cell Biology 49: 13-23.

Rothman JE. 1994. Mechanism of Intracellular Protein-Transport. Nature 372: 55-63.

Samac DA, Peñuela S, Schnurr JA, Hunt EN, Foster-Hartnett D, Vandenbosch KA, Gantt JS. 2011. Expression of coordinately regulated defence response genes and analysis of their role in disease resistance in Medicago truncatula. Molecular Plant Pathology 12: 786-798.

Sanderfoot A. 2007. Increases in the number of SNARE genes parallels the rise of multicellularity among the green plants. Plant physiology 144: 6-17.

Schirawski J, Mannhaupt G, Münch K, Brefort T, Schipper K, Doehlemann G, Di Stasio M, Rössel N, Mendoza-Mendoza A, Pester D, et al. 2010. Pathogenicity determinants in smut fungi revealed by genome comparison. Science 330: 1546-8.

Sekereš J, Pejchar P, Šantrůček J, Vukašinović N, Žárský V, Potocký M. 2017. Analysis of Exocyst Subunit EXO70 Family Reveals Distinct Membrane Polar Domains in Tobacco Pollen Tubes. Plant Physiology 173: 1659-1675.

Serrano MS, De Vita P, Carbonero MD, Fernández F, Fernández-Rebollo P, Sánchez ME. 2012. Susceptibility to Phytophthora cinnamomi of the commonest morphotypes of Holm oak in southern Spain. Forest Pathology 42: 345-347.

Sharma N, Low SH, Misra S, Pallavi B, Weimbs T. 2006. Apical targeting of syntaxin 3 is essential for epithelial cell polarity. Journal of Cell Biology 173: 937-948.

Singh S, Katzer K, Lambert J, Cerri M, Parniske M. 2014. CYCLOPS, A DNA-binding transcriptional activator, orchestrates symbiotic root nodule development. Cell Host and Microbe 15: 139-152.

Sinharoy S, Torres-Jerez I, Bandyopadhyay K, Kereszt A, Pislariu Cl, Nakashima J, Benedito VA, Kondorosi E, Udvardi MK. 2013. The $\mathrm{C} 2 \mathrm{H} 2$ transcription factor regulator of symbiosome differentiation represses transcription of the secretory pathway gene VAMP721a and promotes symbiosome development in Medicago truncatula. The Plant Cell 25: 3584-3601.

Slane D, Reichardt I, El Kasmi F, Bayer M, Jürgens G. 2017. Evolutionarily diverse SYP1 Qa-SNAREs jointly sustain pollen tube growth in Arabidopsis. The Plant Journal 38: 42-49.

Smit P, Raedts J, Prtyanko V, Debelle F, Gough C, Bisseling T, Geurts R. 2005. NSP1 of the GRAS Protein Family is Essential for Rhizobial Nod Factor-Induced Transcription. Science 308: 1789-1791. 
Smith SE, Read D. 2008. Mycorrhizal Symbiosis.

Sollner T, Whiteheart SW, Brunner M, Erdjument-Bromage H, Geromanos S, Tempst P, Rothman JE. 1993. SNAP receptors implicated in vesicle targeting and fusion. Nature 362: 318-324.

Spanu PD, Abbott JC, Amselem J, Burgis TA, Soanes DM, Stüber K, Ver Loren van Themaat E, Brown JKM, Butcher S a, Gurr SJ, et al. 2010. Genome Expansion and Gene Loss in. Science 330: 1543-1546.

Sprent J. 2009. The genus evolutionary structure significance of the by legume Chamaecrista, as determined nodule. 122: 487-492.

Stein A, Weber G, Wahl MC, Jahn R. 2009. Helical extension of the neuronal SNARE complex into the membrane. Nature 460: 525-528.

Stracke S, Kistner C, Yoshida S, Mulder L, Sato S, Kaneko T, Tabata S, Sandal N, Stougaard J, Szczyglowski K, et al. 2002. A plant receptor-like kinase required for both bacterial and fungal symbiosis. Nature 417: 959-962.

Sun J, Miller JB, Granqvist E, Wiley-Kalil A, Gobbato E, Maillet F, Cottaz S, Samain E, Venkateshwaran M, Fort S, et al. 2015. Activation of symbiosis signaling by arbuscular mycorrhizal fungi in legumes and rice. The Plant Cell 27: 823-38.

Svistoonoff S, Hocher V, Gherbi H. 2014. Actinorhizal root nodule symbioses: What is signalling telling on the origins of nodulation? Current Opinion in Plant Biology 20: 11-18.

Tisserant E, Malbreil M, Kuo A, Kohler A, Symeonidi A, Balestrini R, Charron P, Duensing N, Frey NFD, Gianinazzi-Pearson V, et al. 2013. Genome of an arbuscular mycorrhizal fungus provides insight into the oldest plant symbiosis. Proceedings of the National Academy of Sciaences of the United States of America 110: 20117-20122.

Trinick MJ. 1979. Structure of nitrogen-fixing nodules formed by Rhizobium on roots of Parasponia andersonii Planch. Canadian journal of microbiology 25: 565-578.

Trouvelot A, Kough JL, Gianinazzi-Pearson V. 1986. du taux de mycorhization VA d'un système radiculaire. Recherche de méthodes d'estimation ayant une signification fonctionnelle. Mycorhizes: physiologie et génétique: 217-220.

Truchet G, Roche P, Lerouge P, Vasse J, Camut S, Debilly F, Prome JC, Denarie J. 1991. Sulphated lipo-oligosaccharide signals of Rhizobium meliloti elicit root nodule organogenesis in alfalfa. Nature 351: 670-673.

Udvardi M, Poole PS. 2013. Transport and Metabolism in Legume-Rhizobia Symbioses. Annual Review of Plant Biology 64.

Uemura T, Ueda T, Ohniwa RL, Nakano A, Takeyasu K, Sato MH. 2004. Systematic analysis of SNARE molecules in Arabidopsis: dissection of the post-Golgi network in plant cells. Cell structure and function 29: 49-65.

UI-Rehman R, Silva PÂ, Malhó R. 2011. Localization of arabidopsis SYP125 syntaxin in the plasma membrane sub-apical and distal zones of growing pollen tubes. Plant Signaling \& Behavior 6: 665-670.

Van de Velde W, Guerra JCP, De Keyser A, De Rycke R, Rombauts S, Maunoury N, Mergaert P, Kondorosi E, Holsters M, Goormachtig S. 2006. Aging in legume symbiosis. A molecular view on nodule senescence in Medicago truncatula. Plant Physiology 141: 711-20.

Van de Velde W, Zehirov G, Szatmari A, Debreczeny M, Ishihara H, Kevei Z, Farkas A, Mikulass K, Nagy A, Tiricz H, et al. 2010. Plant peptides govern terminal differentiation of bacteria in symbiosis. Science 327: $1122-6$.

Van Velzen R, Holmer R, Bu F, Rutten L, Van Zeijl A, Liu W, Santuari L, Cao Q, Sharma T, Shen D, et al. 2017. Parallel loss of symbiosis genes in relatives of nitrogen-fixing non-legume Parasponia. bioRxiv.

Venkateshwaran M, Jayaraman D, Chabaud M, Genre A, Balloon AJ, Maeda J, Forshey K, den Os D, Kwiecien NW, Coon JJ, et al. 2015. A role for the mevalonate pathway in early plant symbiotic signaling. Proceedings of the National Academy of Sciences of the United States of America 112: 9781-9786.

Vernié T, Kim J, Frances L, Ding Y, Sun J, Guan D, Niebel A, Gifford ML, de Carvalho-Niebel F, Oldroyd GED. 2015. The NIN Transcription Factor Coordinates Diverse Nodulation Programs in Different Tissues of the Medicago truncatula Root. The Plant Cell 27: 3410-3424.

Vijn I, Govers F. 2003. Agrobacterium tumefaciens mediated transformation of the oomycete plant pathogen Phytophthora infestans. Molecular Plant Pathology 4: 459-467. 
Vinardell JM, Fedorova E, Cebolla A, Kevei Z, Horvath G, Kelemen Z, Tarayre S, Roudier F, Mergaert P, Kondorosi A, et al. 2003. Endoreduplication mediated by the anaphase-promoting complex activator CCS52A is required for symbiotic cell differentiation in Medicago truncatula nodules. The Plant Cell 15: 2093-2105.

Volpe V, Giovannetti M, Sun XG, Fiorilli V, Bonfante P. 2016. The phosphate transporters LjPT4 and MtPT4 mediate early root responses to phosphate status in non mycorrhizal roots. Plant, Cell and Environment 39: 660-671.

Walder F, Van Der Heijden MGA. 2015. Regulation of resource exchange in the arbuscular mycorrhizal symbiosis. Nature Plants 1: 1-7.

Wang S, Boevink PC, Welsh L, Zhang R, Whisson SC, Birch PRJ. 2017. Delivery of cytoplasmic and apoplastic effectors from Phytophthora infestans haustoria by distinct secretion pathways. New Phytologist.

Wang Y, Bouwmeester K, de Mortel JE, Shan W, Govers F. 2013. A novel Arabidopsis-oomycete pathosystem: differential interactions with Phytophthora capsici reveal a role for camalexin, indole glucosinolates and salicylic acid in defence. Plant cell and environment 36: 1192-1203.

Wang D, Griffitts J, Starker C, Fedorova E, Limpens E, Ivanov S, Bisseling T, Long S. 2010a. A Nodule-Specific Protein Secretory Pathway Required for Nitrogen-Fixing Symbiosis. Science 327: 1126-1129.

Wang Y, Meng Y, Zhang M, Tong X, Wang Q, Sun Y, Quan J, Govers F, Shan W. 2011. Infection of Arabidopsis thaliana by Phytophthora parasitica and identification of variation in host specificity. Molecular Plant Pathology 12: 187-201.

Wang E, Schornack S, Marsh JF, Gobbato E, Schwessinger B, Eastmond P, Schultze M, Kamoun S, Oldroyd GED. 2012. A common signaling process that promotes mycorrhizal and oomycete colonization of plants. Current Biology 22: 2242-2246.

Wang W, Wen Y, Berkey R, Xiao S. 2009. Specific targeting of the Arabidopsis resistance protein RPW8.2 to the interfacial membrane encasing the fungal Haustorium renders broad-spectrum resistance to powdery mildew. The Plant Cell 21: 2898-913.

Wang B, Yeun LH, Xue JY, Liu Y, An?? JM, Qiu YL. 2010b. Presence of three mycorrhizal genes in the common ancestor of land plants suggests a key role of mycorrhizas in the colonization of land by plants. New Phytologist 186: 514-525.

Wang E, Yu N, Bano SA, Liu C, Miller AJ, Cousins D, Zhang X, Ratet P, Tadege M, Mysore KS, et al. 2014. A $\mathrm{H}+$-ATPase that energizes nutrient uptake during Mycorrhizal Symbioses in Rice and Medicago truncatula. The Plant Cell 26: 1818-1830.

Wang H, Zhuang X, Wang X, Law AHY, Zhao T, Du S, Loy MMT, Jiang L. 2016. A Distinct Pathway for Polar Exocytosis in Plant Cell Wall Formation. Plant Physiology 172: 1003-1018.

Wei X, Chen J, Zhang C, Pan D. 2016. Differential Gene Expression in Rhododendron fortunei Roots Colonized by an Ericoid Mycorrhizal Fungus and Increased Nitrogen Absorption and Plant Growth. Frontiers in Plant Science 7: 1-13.

van West P, Reid B, Campbell TA, Sandrock RW, Fry WE, Kamoun S, Gow NAR. 1999. Green fluorescent protein (GFP) as a reporter gene for the plant pathogenic oomycete Phytophthora palmivora. FEMS Microbiology letters 178: 71-80.

Whisson SC, Boevink PC, Moleleki L, Avrova AO, Morales JG, Gilroy EM, Armstrong MR, Grouffaud S, van West P, Chapman S, et al. 2007. A translocation signal for delivery of oomycete effector proteins into host plant cells. Nature 450: 115-118.

Williamson VM, Hussey RS. 1996. Nematode pathogenesis and resistance in plants. The Plant Cell 8: 17351745.

Xue L, Cui H, Buer B, Vijayakumar V, Delaux P-M, Junkermann S, Bucher M. 2015. Network of GRAS Transcription Factors Involved in the Control of Arbuscule Development in Lotus japonicus. Plant Physiology 167: 854-871.

Yang S-Y, Grønlund M, Jakobsen I, Grotemeyer MS, Rentsch D, Miyao A, Hirochika H, Kumar CS, Sundaresan V, Salamin N, et al. 2012. Nonredundant regulation of rice arbuscular mycorrhizal symbiosis by two members of the phosphate transporter1 gene family. The Plant Cell 24: 4236-51.

Yang X, Zhao W, Hua C, Zheng X, Jing M, Li D, Govers F, Meijer HJG, Wang Y. 2013. Chemotaxis and oospore formation in Phytophthora sojae are controlled by G-protein-coupled receptors with a phosphatidylinositol phosphate kinase domain. Molecular Microbiology 88: 382-394. 
Yano K, Yoshida S, Mueller J, Singh S, Banba M, Vickers K, Markmann K, White C, Schuller B, Sato S, et al. 2008. CYCLOPS, a mediator of symbiotic intracellular accommodation. Proceedings of the National Academy of Sciences of the United States of America 105: 20540-20545.

Yoshizawa AC, Kawashima S, Okuda S, Fujita M, Itoh M, Moriya Y, Hattori M, Kanehisa M. 2006. Extracting sequence motifs and the phylogenetic features of SNARE-dependent membrane traffic. Traffic 7: 11041118.

Yun HS, Kwaaitaal M, Kato N, Yi C, Park S, Sato MH, Schulze-Lefert P, Kwon C. 2013. Requirement of vesicle-associated membrane protein 721 and 722 for sustained growth during immune responses in Arabidopsis. Molecules and Cells: 1-8.

Žárský V, Potocký M. 2010. Recycling domains in plant cell morphogenesis: small GTPase effectors, plasma membrane signalling and the exocyst. Biochemical Society Transactions 38: 723.

Van Zeijl A, Op Den Camp RHM, Deinum EE, Charnikhova T, Franssen H, Op Den Camp HJM, Bouwmeester H, Kohlen W, Bisseling T, Geurts R. 2015. Rhizobium Lipo-chitooligosaccharide Signaling Triggers Accumulation of Cytokinins in Medicago truncatula Roots. Molecular Plant 8: 1213-1226.

Zhang Q, Blaylock LA, Harrison MJ. 2010a. Two Medicago truncatula Half-ABC Transporters Are Essential for Arbuscule Development in Arbuscular Mycorrhizal Symbiosis. The Plant Cell 22: 1483-1497.

Zhang X, Dong W, Sun J, Feng F, Deng Y, He Z, Oldroyd GED, Wang E. 2015a. The receptor kinase CERK1 has dual functions in symbiosis and immunity signalling. The Plant Journal 81: 258-267.

Zhang Y, Liu CM, Emons AMC, Ketelaar T. 2010b. The plant exocyst. Journal of Integrative Plant Biology 52: 138-146.

Zhang X, Pumplin N, Ivanov S, Harrison MJ. 2015b. EXO70I is required for development of a sub-domain of the periarbuscular membrane during arbuscular mycorrhizal symbiosis. Current Biology 25: 2189-2195. 


\section{List of Publications}

Huisman, R., Hontelez, J., Mysore, K.S., Wen, J., Bisseling, T., and Limpens, E. 2016. A symbiosis-dedicated SYNTAXIN OF PLANTS 13ll isoform controls the formation of a stable host-microbe interface in symbiosis. New Phytologist 211:1338-1351.

Huisman, R., Bouwmeester, K., Brattinga, M., Govers, F., Bisseling, T., and Limpens, E. 2015. Haustorium formation in Medicago truncatula roots infected by Phytophthora palmivora does not involve the common endosymbiotic program shared by arbuscular mycorrhizal fungi and rhizobia. Molecular Plant-Microbe Interactions 28:1271-1280.

Huisman, R., and Bisseling, T. 2015. Growth and development: Close relations of secretion and $\mathrm{K}+$. Nature Plants 1.

Maintz, J., Cavdar, M., Tamborski, J., Kwaaitaal, M., Huisman, R., Meesters, C., Kombrink, E., and Panstruga, R. 2014. Comparative analysis of MAMP-induced calcium influx in arabidopsis seedlings and protoplasts. Plant and Cell Physiology 55:1813-1825.

Huisman, R., Ovchinnikova, E., Bisseling, T., and Limpens, E. 2012. Endocytic accommodation of microbes in plants. Pages 271-295 in: Endocytosis in Plants.

Kwaaitaal, M., Huisman, R., Maintz, J., Reinstadler, A., and Panstruga, R. 2011. lonotropic glutamate receptor (iGluR)-like channels mediate MAMP-induced calcium influx in Arabidopsis thaliana. Biochemical Journal 440:355-365.

Schnettler, E., Hemmes, H., Huisman, R., Goldbach, R., Prins, M., and Kormelink, R. 2010. Diverging affinity of tospovirus RNA silencing suppressor proteins, NSs, for various RNA duplex molecules. Journal of Virology 84:11542-11554. 


\section{Acknowledgements}

I am pleased that my work in the lab of MolBi has finally resulted in this PhD thesis. But more important than reaching this milestone, is the time spent along the way. Wageningen, the Radix, and the lab of MolBi are amazing places to work, both because of the science and the people. I really enjoyed my time in the lab, out of the lab, in the coffee room (also out of the lab) and during work discussions (also in the coffee room). With so many helpful and friendly people around, time flies and before you know it four years have passed. Or seven. I want to take this opportunity to name some of the people that greatly contributed to the completion of this thesis:

Erik. I am most grateful to have worked with you. You are an amazing day to day supervisor since you were always there when I needed help, you are patient and critical, a great example on the lab (including lab-bench organization), and incredible fast in returning my manuscripts and chapters after revisions.

Ton. I want to thank you for your trust to let me start without a project, and to give me some extra time in the lab. I admire how you can reduce experiments, ideas and problems to their core, and ask the most relevant questions.

Ton and Erik, I want to thank you both for the freedom and opportunity to form my own hypotheses and chase them. Whether it was a role for SYP121 in infection thread formation or the involvement of SYP132 in sanctioning, I turned out to be wrong. Nevertheless, looking back I feel that this has been the most valuable experience in my PhD.

For the work described in Chapter 2, I owe gratitude to Klaas and Francine. Klaas, you never ran out of possible solutions for any difficulties we met working with a wide range of pathogens. your positive mindset was of great help to me when I felt nothing would ever work. Francine, I also want to thank you for being my external supervisor.

Tian, Jan Ho, Jiangyong, Jelle and Peng. The mycorrhiza squad. Life is so much easier when your colleagues do the same type of experiments and share protocols. Tian, especially you helped me out many times using laser capture microdissection and solving problems with the mycorrhization assay. Thanks for joining me on stage as my paranymph.

Henk, my first real exposure to MolBi was as a toolbox student. From then onwards, I greatly enjoyed talking to you about science, life and music, your sense of humour, and occasional wisdom. Together with Olga and Marijke you made the lunchbreaks always interesting. 
Elena, when someone asks you what concentration of FM4-64 to use, they should be prepared for a monologue of at least one hour. I quickly learned to appreciate these conversations and the advice that comes with them about science and microscopy.

Rene, you always gave helpful advice on moments when the project seemed to be stuck. It is good to have a scientist like you around. Not completely unimportant either, you alerted me on a vacancy for some postdoc position in England. Thank you!

Jan V, whatever my gel looked like, it was always nice to show it to you. After I admitted that I again did not load an uncut vector control (seriously, who does?), the conversation would proceed to music. Although there is no one with a similar taste as you, we can agree on Steely Dan.

Maria and Marie-Jose. Where would the lab be without you? Thanks for all your Administrative work, organization, ordering and time writing.

Marijke B, Illias, Kate, Pieter and Pelle, Thanks for working with me on your thesis. Besides your contribution of useful data and setting up experiments, I enjoyed working together with all of you, as working together on a project is just more fun than working alone.

Sabine, Adam, Arjan, Tingting, Bandan, Jeroen. I really enjoyed the evenings we spent having diner and drinking beer at the Vlaamse Reus together. Arjan, thanks for joining me on stage as my paranymph.

Paulus, Lenny, Kees, Pascal, Thijs. There is no better way to release energy than to spend a Monday evening with you lot. Although it did not always benefit the Tuesday productivity, It sure was fun. Unfortunately our band is currently experiencing some hiatus, I'm confident that we'll break through one day.

A special thanks to all the bands that I played in during my PhD: LA 166, Let it Beatles, Sir Pfeiffer and the yo'mama Jokes, de ROB-band as well as a brief youtube carreer with Titis and Martinus.

All other colleagues from MolBi, Cell Biology or Developmental Biology who made the coffee breaks the event of the day to look forward to: Joan, Huchen, Guiling, Defeng, Fengjiao, Xu, Rens, Jieyu, Yinshan, Luuk, Yuda, Asma, Amina, Ole, Maryam, Trupti, Robin, Kerstin, Sidney, Simon, Carolien, Kana, Comfort, Sergey, Aleksandr, Sjef, Vid, Rik, Arend, Gerben, Alessandra, Chunting, Ludmilla, Jac, Jorge, Steven, Bas, Wenkun, Viola, Renze, Ikram, Du, Ben, Serena, Aniek, Elysa, Juliane, Chris, Han, Marcel, Thijs, Norbert, Hannie and Henk K.

John, Paul, George and Ringo. Thanks for composing such wonderful music that provided a soundtrack for much of my PhD.

Tot slot, Papa, Mama, Martine, Hilbert, Jordi, Lize, Tobie en Keetie, en Sociëteit 'De Flippies' bedankt voor alle steun en gezelligheid in het weekend. 


\section{Education Statement of the Graduate School \\ Experimental Plant Sciences}

Issued to: Rik Huisman

Date: $\quad 16$ February 2018

Group: $\quad$ Laboratory of Molecular Biology

University: Wageningen University \& Research

\section{1) Start-up phase}

- First presentation of your project

Title: Intracellular accomodation of microbes in Medicago truncatula

- Writing or rewriting a project proposal

Title: Intracellular accomodation of microbes in Medicago truncatula

- Writing a review or book chapter

Endocytic accomodation of microbes in plants. In: Šamaj J. (eds) Endocytosis in Plants. Springer, Berlin,

Heidelberg. DOI: https://doi.org/10.1007/978-3-642-32463-5 14

- MSc courses

Laboratory use of isotopes

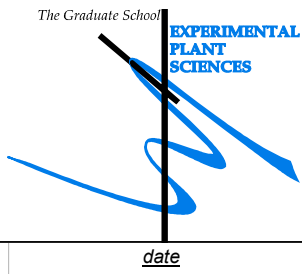

04 Feb 2011

06 Jul 2012

26 Jun 2012

11.5 credits $^{*}$

\section{2) Scientific Exposure}

- EPS PhD student days

EPS PhD student day, Wageningen, NL

EPS PhD student day, Leiden, NL

EPS PhD Student Days 'Get2Gether', Soest, NL

- EPS theme symposia

EPS Theme 1 Symposium 'Developmental Biology of Plants', Leiden, NL

EPS Theme 1 Symposium 'Developmental Biology of Plants', Wageningen, NL

EPS Theme 2 Symposium 'Interactions between Plants and Biotic Agents', Wageningen, NL

EPS Theme 1 Symposium 'Developmental Biology of Plants', Leiden, NL

EPS Theme 1 Symposium 'Developmental Biology of Plants', Wageningen, NL

EPS Theme 1 Symposium 'Developmental Biology of Plants', Leiden, NL

EPS Theme 1 Symposium 'Developmental Biology of Plants', Wageningen, NL

EPS Theme 1 Symposium 'Developmental Biology of Plants', Leiden, NL

- National meetings (e.g. Lunteren days) and other national platforms

Annual meeting 'Experimental Plant Sciences', Lunteren, NL

Annual meeting 'Experimental Plant Sciences', Lunteren, NL

Annual meeting 'Experimental Plant Sciences', Lunteren, NL

Annual meeting 'Experimental Plant Sciences', Lunteren, NL

Annual meeting 'Experimental Plant Sciences', Lunteren, NL

Annual meeting 'Experimental Plant Sciences', Lunteren, NL

Annual meeting 'Experimental Plant Sciences', Lunteren, NL

Subtotal Start-up Phase

$\underline{\text { date }}$

20 May 2011

29 Nov 2013

29-30 Jan 2015

20 Jan 2011

19 Jan 2012

10 Feb 2012

17 Jan 2013

24 Jan 2014

08 Jan 2015

21 Jan 2016

28 Feb 2017

04-05 Apr 2011

02-03 Apr 2012

22-23 Apr 2013

14-15 Apr 2014

13-14 Apr 2015

11-12 Apr 2016

10-11 Apr 2017

- Seminars (series), workshops and symposia

Invited seminar Emma Granqvist: 'Calcium signalling in plant-microbe interactions'

Workshop: 'New developments in plant endomembrane biology'

Invited seminar Joop Vermeer: 'Endodermal cell responses are essential for lateral root initialtion and outgrowth'

Invited seminar Saïd Amrar: 'Plant research in the Sahara'

Mini symposium 'Frontiers in Plant Morphogenesis'

EPS Flying Seminar Ralph Panstruga

Invited seminar Andrew Sudgen: 'Writing for high impact journals'

Invited seminar Andreas Niebel

Invited seminar David Weller

Symposium 'Plant Developmental Biology'

Invited seminar Jos Raaijmakers

Invited seminar Toby Kiers

Mini symposium Phytopathology

EPS Mini Symposium 'Root development and rhizosphere'

Invited seminar David Salt: 'Landscape ionomics: The functional genomics of ecologically adaptive

Invited seminar Marc-André Selosse: 'Mycoheterotrophy and mixotrophy: plants eating mycorrhizal fungi'

Invited seminar Pierre-Marc Delaux: 'Evolution of symbiotic gene networks in land plants'

Invited seminar Daniel von Wangenheim: 'Long-term Imaging of Root Development using Light-Sheet

Fluorescence Microscopy'

Invited seminar Hans Thordal-Christensen: 'Membrane trafficking in plant cells attacked by powdery

mildew fungi'

Farewell Symposium of Ton Bisseling

Invited seminar Gerd Jürgens

06 Sep 2011

15 Jun 2012

31 Aug 2012

08 Nov 2012

13 Nov 2012

04 Dec 2012

08 Feb 2013

28 Mar 2013

25 Sep 2013

14 Oct 2013

07 Jan 2014

11 Mar 2014

24 Nov 2014

20 May 2015

27 Oct 2015

26 Nov 2015

08 Apr 2016

11 Oct 2016

12 Dec 2016

08 Feb 2017

11 May 2017 


\begin{tabular}{|c|c|c|}
\hline$D$ & $\begin{array}{l}\text { Seminar plus } \\
\text { International symposia and congresses } \\
\text { RMS conference for Botanical microscopy, Wageningen, NL } \\
\text { 10th European Nitrogen fixation conference, Munich, Germany } \\
\text { 1st International Moleccular Mycorrhiza Meeting, Munich Germany } \\
\text { XVI International congres on MPMI, Rhoode Island, Greece } \\
\text { 2nd International Moleccular Mycorrhiza Meeting, Cambridge, UK } \\
\text { 36th New Phytologist Symposium: Cell biology at the plant-microbe interface, Munich, Germany } \\
\text { Signaling at the Plant-Soil Interface } \\
\text { 3rd International Moleccular Mycorrhiza Meeting, Toulouse, France } \\
\text { Presentations } \\
\text { Talk: } \\
\text { Plant Science Slam Lunteren, NL } \\
\text { Symposium developmental biology } \\
\text { EPS Theme } 1 \text { Symposium } \\
\text { Annual meeting 'Experimental Plant Sciences', Lunteren } \\
\text { 36th New Phytologist Symposium: Cell biology at the plant-microbe interface } \\
\text { Signaling at the Plant-Soil Interface } \\
\text { 3rd International Moleccular Mycorrhiza Meeting } \\
\text { Poster: } \\
\text { Annual meeting 'Experimental Plant Sciences', Lunteren, NL } \\
\text { XVI International congres on MPMI } \\
\text { 2nd International Moleccular Mycorrhiza Meeting } \\
\text { 3rd International Moleccular Mycorrhiza Meeting } \\
\text { IAB interview } \\
\text { Meeting with a member of the International Advisory Board of EPS } \\
\text { Excursions } \\
\text { Company visit Genetwister and In2Care }\end{array}$ & $\begin{array}{c}\text { 02-03 Apr } 2012 \\
\text { 14 Oct } 2013 \\
24 \text { Jan } 2014 \\
\text { 14 Apr } 2015 \\
\text { 30 Nov } 2015 \\
\text { 14 Sep } 2016 \\
\text { 27 Jul } 2017 \\
\text { 22-23 Apr } 2013 \\
\text { 06-10 Jul } 2014 \\
\text { 02-04 Sep } 2015 \\
\text { 26-28 Jul } 2017 \\
\\
\text { 03 Dec } 2014 \\
\text { 19 Sep } 2014\end{array}$ \\
\hline
\end{tabular}

\begin{tabular}{|l|r|}
\hline 3) In-Depth Studies & date \\
\hline $\begin{array}{l}\text { EPS courses or other PhD courses } \\
\text { Mournal club } \\
\text { Jourticipation in a literature discussion group } \\
\text { Individual research training }\end{array}$ & $07-11$ May 2012 \\
\hline
\end{tabular}

Subtotal In-Depth Studies

4.5 credits*

\begin{tabular}{|c|c|}
\hline $\begin{array}{l}\text { 4) Personal development } \\
\text { Skill training courses } \\
\text { Scientific writing } \\
\text { Career Orientation } \\
\text { PhD workshop carousel } \\
\text { Adobe InDesign course } \\
\text { The awareness training on biological safety 'Better Safe Than Sorry' } \\
\text { Organisation of PhD students day, course or conference } \\
\text { Membership of Board, Committee or PhD council }\end{array}$ & $\begin{array}{c}\text { date } \\
12 \text { Dec-18 Feb } 2014 \\
28 \text { Feb-28 Mar } 2014 \\
\text { 02 Jul } 2014 \\
\text { 07-08 Nov } 2016 \\
\text { 28 Jul } 2016\end{array}$ \\
\hline Subtotal Personal Development & 4.3 credits* \\
\hline TOTAL NUMBER OF CREDIT POINTS* & 51,5 \\
\hline
\end{tabular}

Herewith the Graduate School declares that the $\mathrm{PhD}$ candidate has complied with the educational requirements set by the Educational Committee of EPS which comprises of a minimum total of 30 ECTS credits

${ }^{*}$ A credit represents a normative study load of 28 hours of study. 
